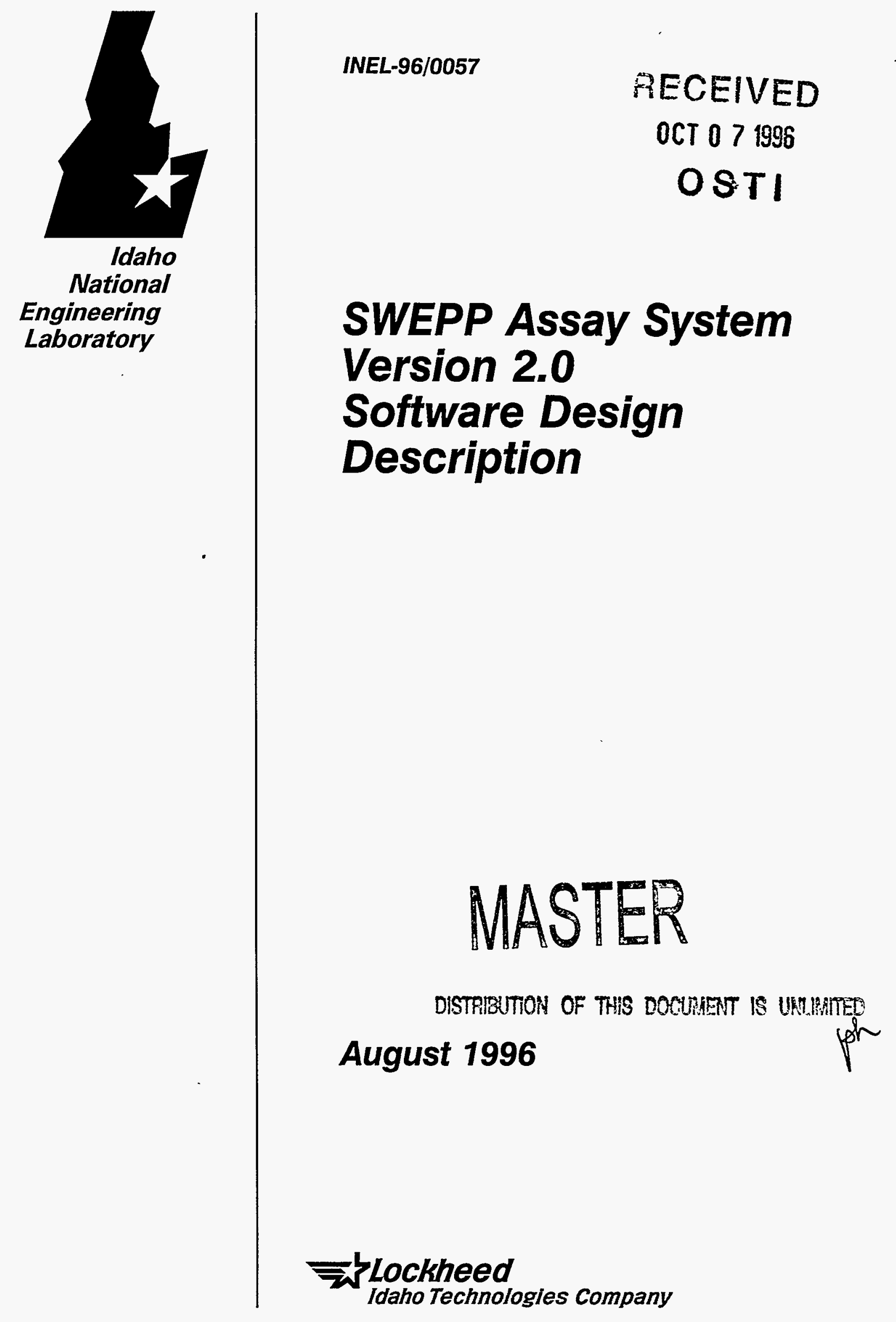




\section{SWEPP Assay System Version 2.0 Software Design Description}

L.V. East

E.S. Marwil

Published August 1996

Idaho National Engineering Laboratory

Lockheed Martin Idaho Technologies Company

Idaho Falls, Idaho 83415

Prepared for the

U.S. Department of Energy

Office of Environmental Restoration and Waste Management

Under DOE Idaho Operations Office

Contract DE-AC07-761D01570 


\section{DISCLAMMER}

Portions of this document may be illegible in electronic image products. Images are produced from the best available original document. 


\section{DISCLAIMER}

This report was prepared as an account of work sponsored by an agency of the United States Government. Neither the United States Government nor any agency thereof, nor any of their employees, makes any warranty, express or implied, or assumes any legal liability or responsibility for the accuracy, completeness, or usefulness of any information, apparatus, product, or process disclosed, or represents that its use would not infringe privately owned rights. Reference herein to any specific commercial product, process, or service by trade name, trademark, manufacturer, or otherwise does not necessarily constitute or imply its endorsement, recommendation, or favoring by the United States Government or any agency thereof. The views and opinions of authors expressed herein do not necessarily state or reflect those of the United States Government or any agency thereof. 
INEL-96/0057

\section{Acknowledgments}

The comments and suggestions provided by the RWMC/SWEPP Operations Personnel under the direction of G. K. Tedford during the development and testing of this software are gratefully acknowledged. Extensive testing and evaluation of the software during development of Version 2.0 was provided by $G$. W. Twedell.

\section{Approvals}

Approval signatures for this document are contained in DAR-EO-RS-2799 on file in the Document Control Office of the INEL Radioactive Waste Management Complex. AF 
. 


\section{Contents}

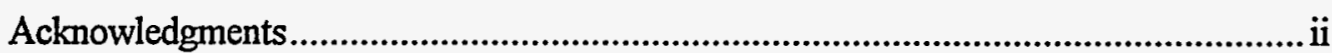

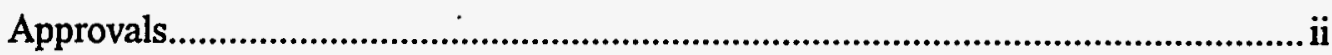

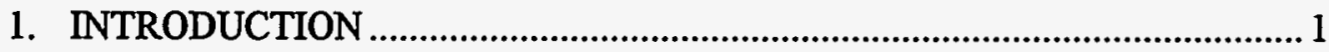

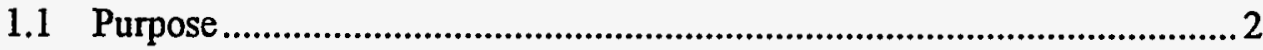

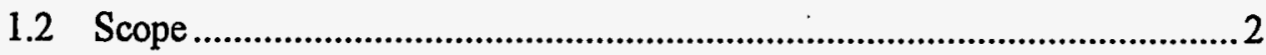

1.3 Definitions, Acronyms, and Abbreviations ............................................ 2

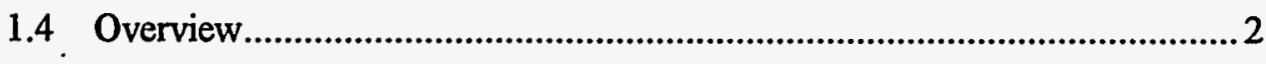

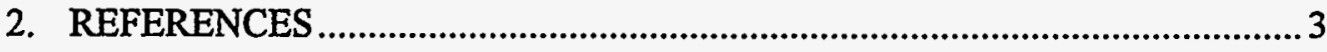

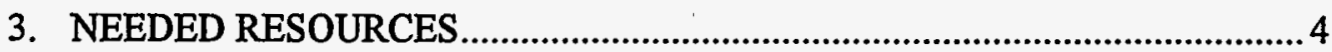

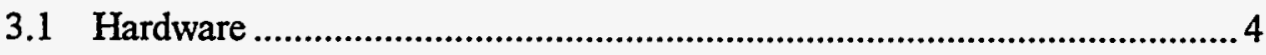

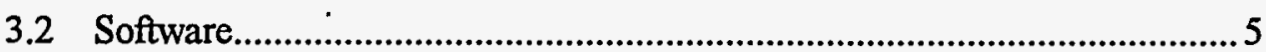

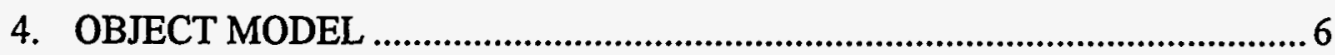

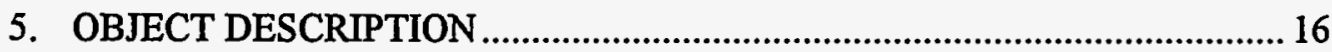

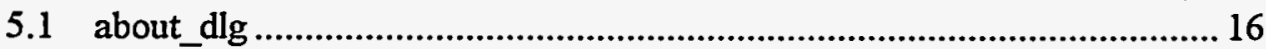

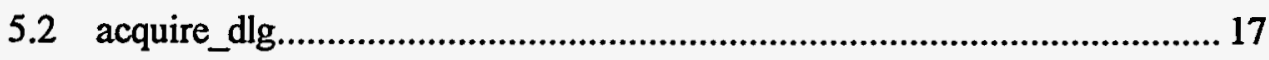

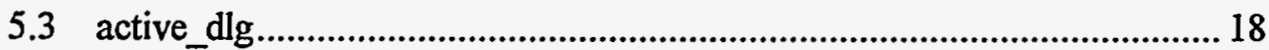

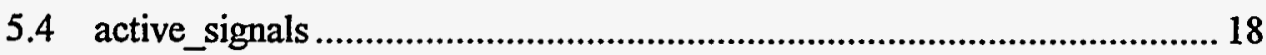

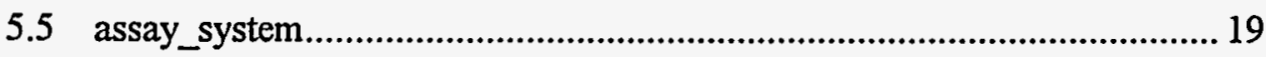

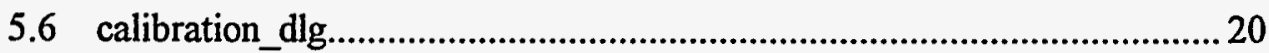

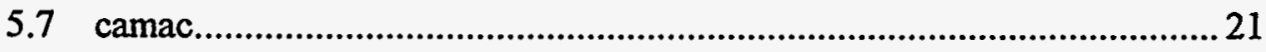

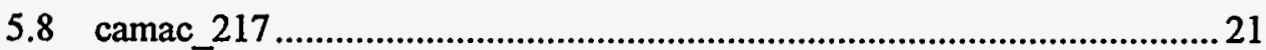

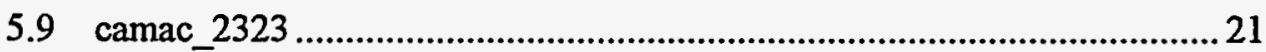

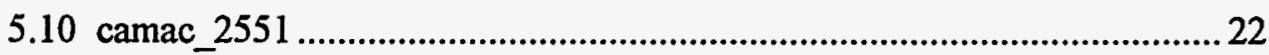

5.11 camac_450

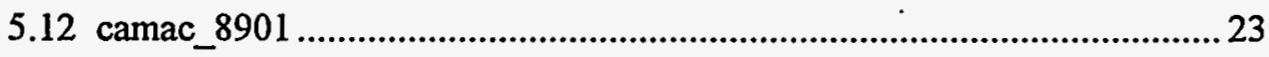

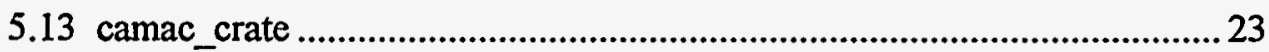

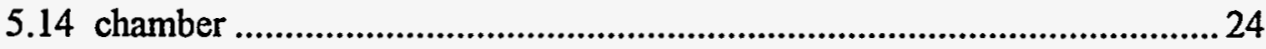

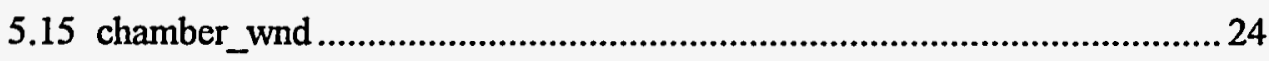

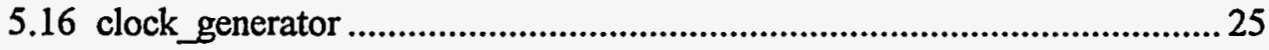

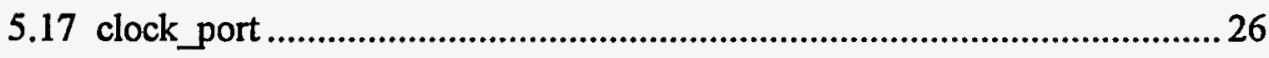

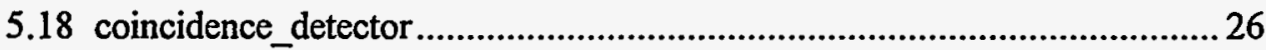

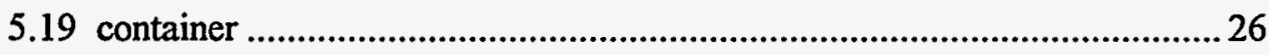

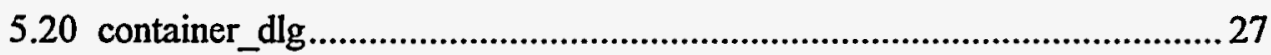

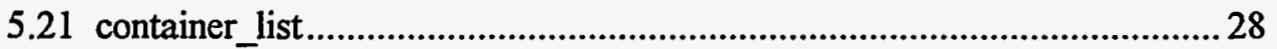

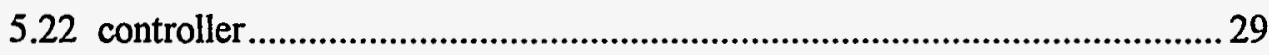

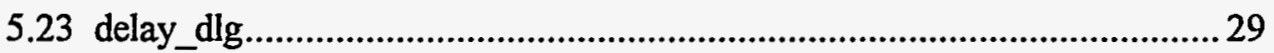




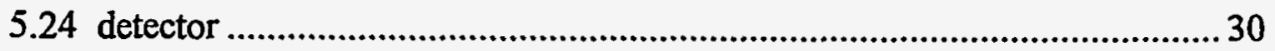

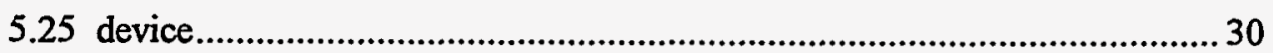

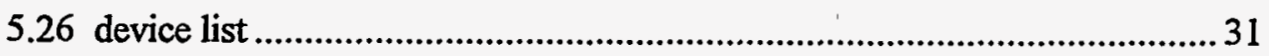

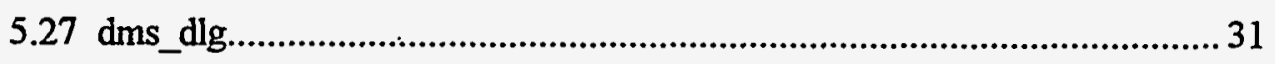

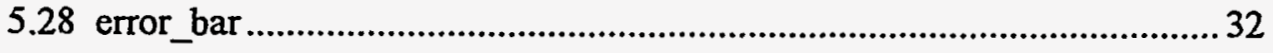

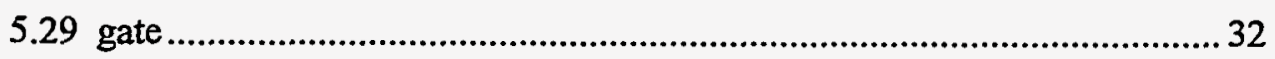

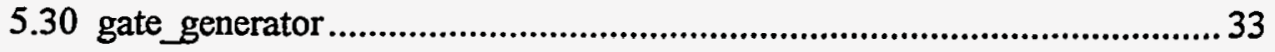

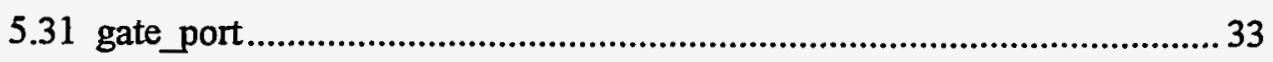

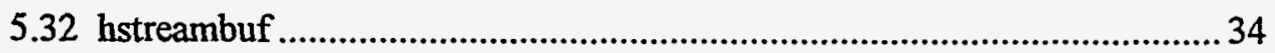

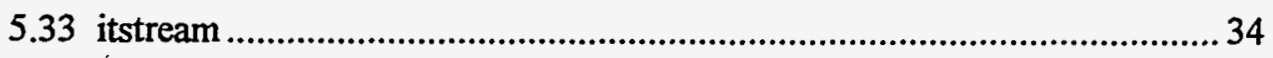

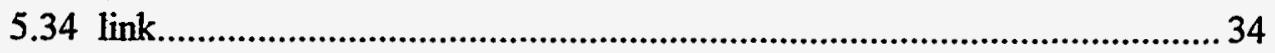

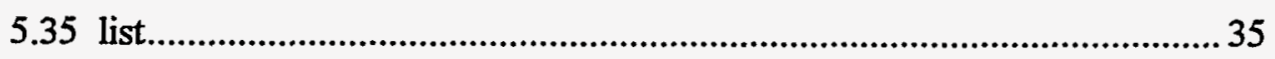

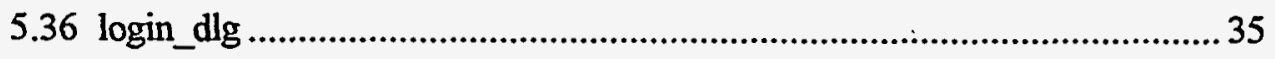

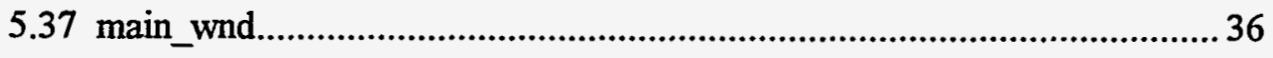

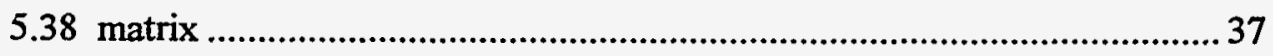

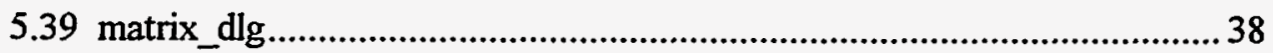

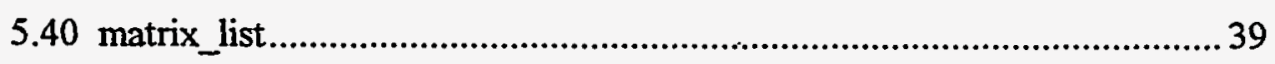

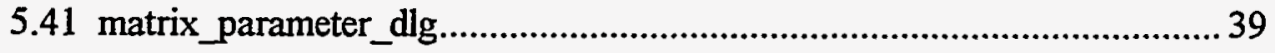

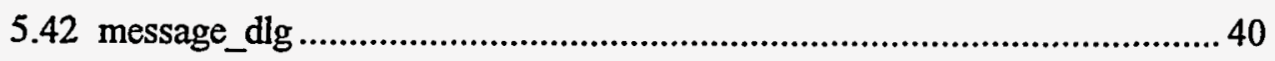

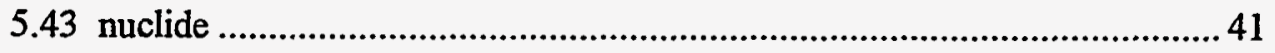

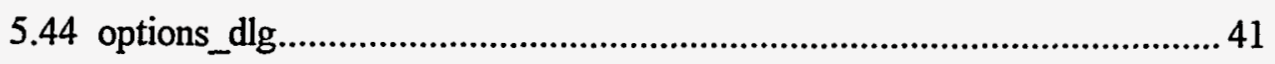

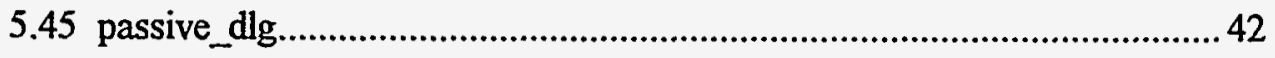

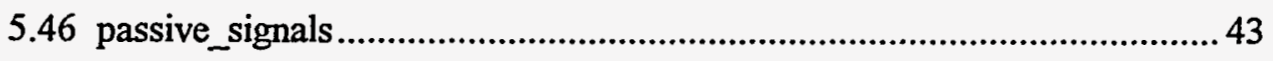

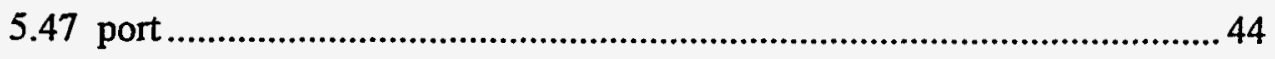

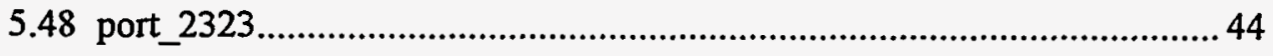

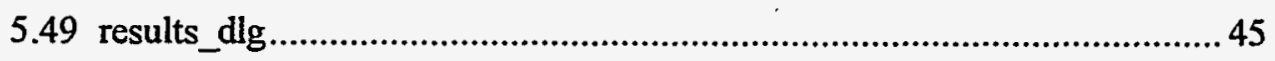

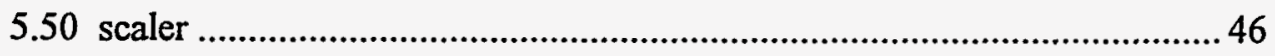

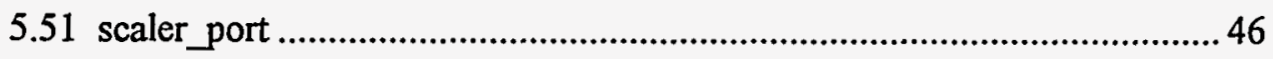

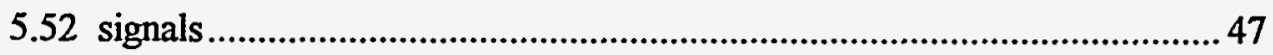

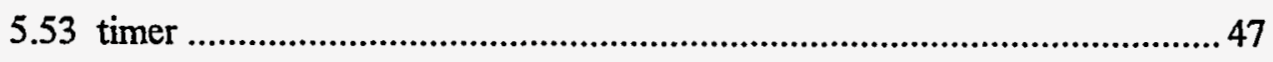

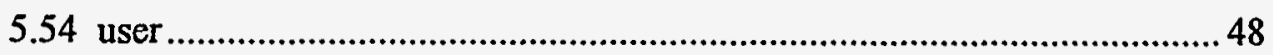

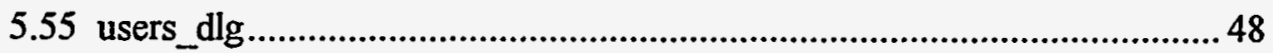

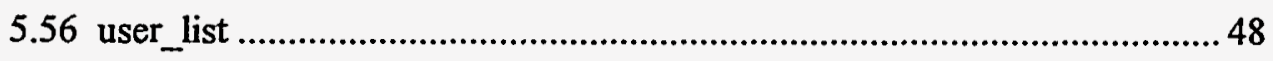

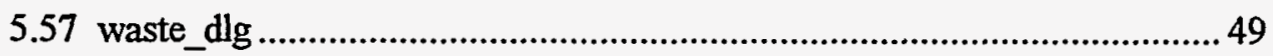

6. REQUIREMENTS TRACEABILITY ........................................................ 51

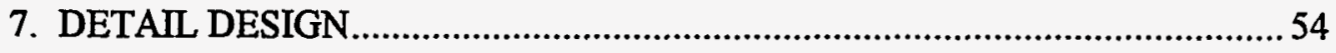

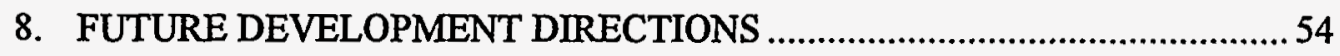

Appendix A - Include Files..........................................................................

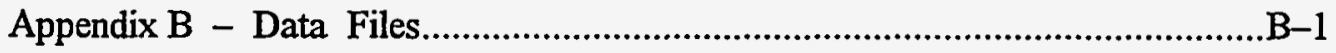




\section{List of Figures}

1. Assay system object model, level 1. ............................................................

2. Assay system object model, level 1.1. ............................................................... 8

3. Assay system object model, level 1.2. ............................................................. 9

4. Assay system object model, level 1.3............................................................... 10

5. Assay system object model, level 1.4. ............................................................. 11

6. Assay system object model, level 1.4.1......................................................... 12

7. Assay system object model, level 1.4.1.1........................................................... 13

8. Assay system object model, level 1.4.1.2.......................................................... 14

9. Assay system object model, level 1.4.1.3.......................................................... 15

10. Assay system object model, level 1.4.2 .......................................................... 15

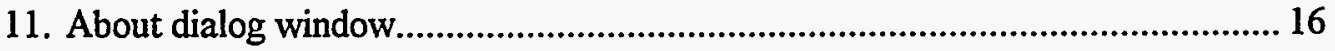

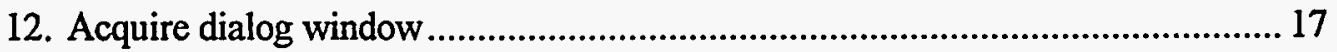

13. Active dialog (data) window ........................................................................ 18

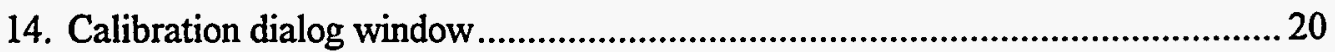

15. Assay chamber window and child windows .................................................... 25

16. Container dialog window .............................................................................. 28

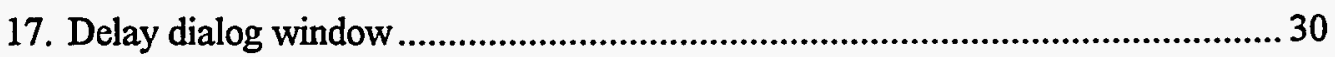

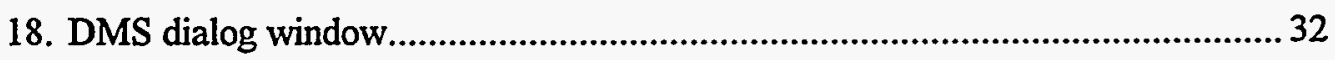

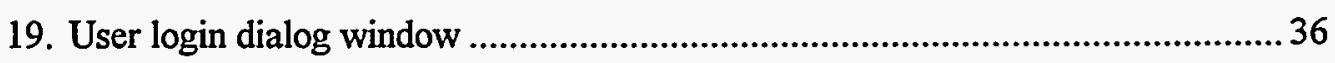

20. Main window with one chamber as a child window ............................................ 37

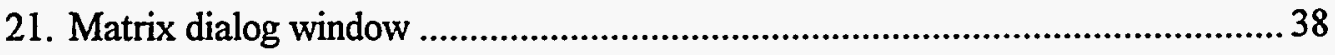

22. Matrix parameter dialog window ..................................................................... 40

23. Message dialog window................................................................................... 41

24. Processing options (assay times) dialog window................................................. 42

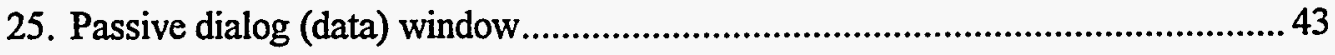

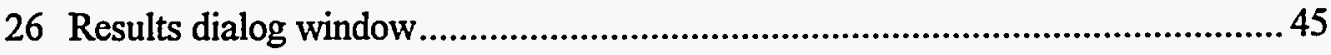

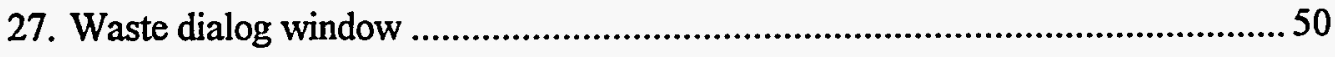

\section{List of Tables}

1. CAMAC crate module configuration................................................................... 4

2. Chamber configuration files.............................................................................6

3. Requirements traceability matrix. ………........................................................5 
INEL-96/0057

\section{SWEPP Assay System Software Design Description}

\section{INTRODUCTION}

The Idaho National Engineering Laboratory (INEL) Stored Waste Examination Pilot Plant (SWEPP) operations staff use nondestructive analysis methods to characterize the radiological contents of contact-handled radioactive waste containers. Containers of waste from Rocky Flats Environmental Technology Site and other Department of Energy (DOE) sites are currently stored at SWEPP. Before these containers can be shipped to the Waste Isolation Pilot Plant (WIPP), SWEPP must verify compliance with storage, shipping, and disposal requirements. This program has been in operation since 1985 at the INEL Radioactive Waste Management Complex (RWMC).

One part of the SWEPP program measures neutron emissions from the containers and estimates the mass of plutonium and other transuranic (TRU) isotopes present. A Passive/Active Neutron (PAN) assay system developed at the Los Alamos National Laboratory [1] ${ }^{1}$ is used to perform these measurements. A computer program named NEUT2 was originally used to perform the data acquisition and reduction functions for the neutron measurements. This program was originally developed at Los Alamos and extensively modified by a commercial vendor of PAN systems and by personnel at the INEL. NEUT2 uses the analysis methodology outlined in [1], but no formal documentation exists on the program itself. The SWEPP Assay System (SAS) computer program replaced the NEUT2 program in early 1994. The SAS software was developed using an "object model" approach and is documented in accordance with American National Standards Institute (ANSI) and Institute of Electrical and Electronic Engineers (IEEE) standards. The new program incorporates the basic analysis algorithms found in NEUT2. Additional functionality and improvements include a graphical user interface, the ability to change analysis parameters without program code modification, an "object model" design approach and other features for improved flexibility and maintainability.

This software design description (SDD) presents the SAS computer program Version 2.0 design and supersedes the original SAS software design description [2]. Companion documents include a software requirements specification [3], a software verification and validation plan [4], a software test plan and report [5], and a software configuration management plan [6]. Development of this technology is expected to continue following the Version 2.0 release.

Version 1.0 of the SAS software was developed by staff at the INEL Scientific Computing unit under the technical direction of the Nuclear and Radiation Physics unit. Version 2.0 is under development by staff from the INEL Nuclear Physics and Radiation Chemistry Department, with documentation and testing assistance provided by personnel from the Software and Electronics Department and the Engineering Analysis Department. Funding for this work has been provided through the INEL TRU Waste Programs Department.

\footnotetext{
${ }^{1}$ References cited in this document are listed in Section 2.
} 


\subsection{Purpose}

This SDD covers SAS internal and external interfaces. The primary purpose of this design description is to provide a foundation for SAS development and verification and validation conformable to INEL/RWMC quality requirements [7].

The intended audiences for this specification are the developers and verification and validation analysts for the SAS software. In addition, this SDD provides a basis for understanding between the developers and the users of SAS.

This description follows the design description format and content guideline suggested by IEEE software engineering standards [8]. Methods employed are those commonly associated with Object Oriented Design, specifically the Rumbaugh et al. [9] implementation of object models and state transition diagrams. A computer program called MacAnalyst/MacDesigner [10] was employed as a Computer Aided Software Engineering (CASE) tool to provide automation of these methods during the Version 1.0 design.

\subsection{Scope}

The design description records the division of the software system into design entities. It describes the way the system has been structured and the purpose and function of each entity. For each entity, it provides a detailed description in terms of a Program Design Language (PDL). The design specifies the relationships between entities and the interfaces used.

\subsection{Definitions, Acronyms, and Abbreviations}

Definitions, acronyms, and abbreviations typical to an SDD and to the life cycle approach to software development in general are provided in Reference [11]. The following terms are specific to this document:

Design entity - An element (component) of a design that is structurally and functionally distinct from other elements and that is separately named and referenced.

Entity attribute - A named characteristic or property of a design entity. It provides a statement of fact about the entity.

MFC - Microsoft Foundation Class.

\subsection{Document Overview}

Section 2 lists the references for this document. Section 3 identifies the hardware and software resources required. Section 4 presents the object model for the SAS software, and section 5 gives the descriptions for each object. Section 6 provides the requirements traceability matrix. Section 7 presents detailed design information. 


\section{REFERENCES}

[1] J.T. Caldwell, et. al., The Los Alamos Second-Geneation System for Passive and Active Neutron Assays of Drum-Size Containers, LA-10774-MS, Los Alamos National Laboratory, September 1986.

[2] S.D. Matthews and E.S. Marwil, SWEPP Assay System Design Description, EGG-RAAM10424, EG\&G Idaho,Inc. September 1993.

[3] S.D. Matthews, L.V. East, E.S. Marwil and J.J. Ferguson, SWEPP Assay System Version 2.0 Software Requirements Specification, INEL-96/0056, Lockheed Idaho Technologies Co., March 1996.

[4] S.D. Matthews, SWEPP Nondestructive Assay Software Verification and Validation Plan, INEL-96/-0008, Lockheed Idaho Technologies Co., March 1996.

[5] J.J. Ferguson and T.K. Overlin, SWEPP Assay System Version 2.0 Software Test Plan and Report, INEL-96/0058, Lockheed Idaho Technologies Co., April 1966.

[6] J.J. Ferguson, K.D.Boyd and E.S. Marwil, SWEPP Assay System Version 2.0 Software Configuration Management Plan, INEl-96/0007, Lockheed Idaho Technologies Co., March 1996, (to be developed).

[7] "RWMC Quality Assurance Project Plan (QAPjP)", INEL-94/0084, 1994.

[8] ANSI/IEEE Std. 1016-1987, IEEE Recommended Practice for Software Design Descriptions, Institute of Electrical and Electronic Engineers, Inc.

[9] James Rumbaugh et al., Object-Oriented Modeling and Design. Prentice-Hall, New York, 1991.

[10] "MacAnalyst, Release 3.2, "Excel Software, Marshalltown, Iowa, 1991.

[11] ANSIIEEE Std. 610.1a-10990, IEEE Standard Glossary of Software Engineering Terminology, Institute of Electrical and Electronic Engineers, Inc.

[12] National Instruments Corp., Austin, Texas.

[13] G.F. Knoll, Radiation Detection and Measurement, John Wiley and Sons, New York, NY, 1979.

[14] H.W. Coleman and W.G. Steel, Experimentation and Uncertainty Analysis for Engineers, John Wiley and Sons, New York, NY, 1989.

[15] K.D. Welker, B.C. Hong, G.V. Miller and B.J. Colborn, Stored Waste Examination Pilot Plant Assay System Metrics Research Study, INEL-95/0391, Lockheed Idaho Technologies Co., September 1995.

[16] Microsoft Corporation, Class Library Reference for the Microsoft Foundation Class Library, Document No. DB35743-0193, 1993. 


\section{NEEDED RESOURCES}

\subsection{Hardware}

SAS must execute in the current hardware environment. The current computer is an IBM compatible PC with an INTEL 486 CPU. A mouse (or other MS-Windows supported pointing device), VGA color monitor, and Hewlett Packard LaserJet III compatible printer are also required. At least eight megabytes of MS-Windows accessible memory, a floppy disk, and a hard disk with at least ten megabytes of free space are required. In addition, a free serial port is required for SAS communication with the SWEPP Data Management System (DMS).

The SAS program accesses signal data from a Computer Automated Measurement and Control (CAMAC) instrument crate. The required crate, modules, and controller shall be those currently in use at SWEPP. The PC requires an AT-GPIB controller card from National Instruments Corporation [12] to manage the interface to the CAMAC crate through the crate controller. Table 1.

The current configuration of the CAMAC crate by station number and module number are given in

Table 1. CAMAC crate module configuration.

\begin{tabular}{lcl}
\hline Station & Module type & \multicolumn{1}{c}{ Module name } \\
\hline $01-02$ & 2323 & LeCroy 2323A Gate Generator \\
$03-04$ & 2323 & LeCroy 2323A Gate Generator \\
05 & 2551 & LeCroy 2551 Scaler \\
06 & 2551 & LeCroy 2551 Scaler \\
$07-08$ & 2323 & LeCroy 2323A Gate Generator \\
$09-10$ & 2323 & LeCroy 2323A Gate Generator \\
11 & 2551 & LeCroy 2551 Scaler \\
12 & 2551 & LeCroy 2551 Scaler \\
13 & 450 & DSP 450 Gated Quad Scaler \\
$14-15$ & 2323 & LeCroy 2323A Gate Generator \\
$16-17$ & 2323 & LeCroy 2323A Gate Generator \\
18 & 2551 & LeCroy 2551 Scaler \\
19 & 2551 & LeCroy 2551 Scaler \\
20 & 450 & DSP 450 Gated Quad Scaler \\
21 & 217 & Jorway 217 Gated Clock Generator \\
22 & 202 & Jorway 202 Dataway Display \\
23 & 2551 & LeCroy 2551 Scaler \\
$24-25$ & 8901 & LeCroy 8901A GPIB Interface
\end{tabular}




\subsection{Software}

\subsubsection{Language and Tools}

Version 2.0 of the SAS software was developed using the Microsoft Visual C++ V1.51 development environment for Microsoft Windows 3.1. The device driver for the AT-GPIB controller (GPIB.DLL) and the interface library (GPIB.LIB) are licensed from National Instruments [12].

\subsubsection{Necessary Files for Execution}

\section{Data Files}

These data files are described briefly here. Each file is listed in Appendix B.

\section{System configuration files}

AS.INI - the system initialization file. The program's root directory; user and error logging files, printer, serial port configuration, files containing the user list, the container list, and the matrix list, and the chamber initialization files are defined in this file.

ASSAYSYS.INI ${ }^{2}$ - program initialization file. This file resides in the default Windows directory and is used to save window size, placement and color information. This file is automatically created the first time the program is executed and updated each time the program exits. It is not listed in the appendix.

DAC.INI - the configuration file for the drum assay chamber. This file identifies the name of the assay chamber, the defaults for printing the summary report, applying passive background correction and automatically saving data, customization parameters for background integrity checks and other functions, the directories for this assay chamber, and the files for this assay chamber.

CAC.INI - the configuration file for the crate assay chamber. This file identifies the name of the assay chamber, the defaults for printing the summary report, applying passive background correction and automatically saving data, customization parameters for background integrity checks and other functions, the directories for this assay chamber, and the files for this assay chamber.

USER.LST - the list of users who have access to the system. This file is not listed in the appendix.

CONTAIN.LST - the list of container types and volumes that the assay system can process.

MATRIX.LST - the list of matrix types and attributes that the assay system can process. The attributes are those which can be modified by a user with Physicist access level.

NUCLIDE.DAT - the data file that defines the physical properties of the plutonium, uranium and americium nuclides that are used by the SAS.

DEFAULT.GAM - the default distribution of the plutonium isotopes. This file is used if no other gamma data is available for the container being processed.

\footnotetext{
${ }^{2}$ The name of this file may change; it will be the same as the executable file name with the extension.INI. Unlike AS.INI, DAC.INI, etc., this is a "true" MS Windows initialization file.
} 


\section{Chamber configuration files}

The chamber configuration files are identified in Table 2. The options data file defines the default processing options for background count time, active pulses and passive count times for both verification and production runs. The calibration file defines the chamber attributes (detector efficiencies, zero matrix calibration factors, and the mass fractions of plutonium used for calibration). The correlation data file defines the coefficients for the correlations taken from the NEUT2 program. The chi-square file defines the parameters for the Chi-square tests. Two CAMAC configuration files describe the CAMAC crate configuration, one for the active data acquisition and one for the passive data acquisition. Finally, the files for the current passive background data and current verification data, while not strictly part of the configuration, are listed for completeness.

Table 2. Chamber configuration files.

\begin{tabular}{lll}
\hline \multicolumn{1}{c}{ Description } & Drum Assay Chamber & Crate Assay Chamber \\
\hline Processing options & OPTIONS.DAC & OPTIONS.CAC \\
Chamber calibration & CALIB.DAC & CALIB.CAC \\
Correlation coefficients & CORREL.DAC & CORREL.CAC \\
Chi-square parameters & CHISQ.DAC & CHISQ.CAC \\
Active CAMAC configuration & ACTCRATE.DAC & ACTCRATE.CAC \\
Passive CAMAC crate configuration & PASCRATE.DAC & PASCRATE.CAC \\
Current passive background data & BACKGRND & BACKGRND \\
Current verification data & 000496 & 000496 \\
\hline
\end{tabular}




\section{OBJECT MODEL}

This section provides the object model, using Rumbaugh's notation [9]. The object model, shown on the following pages, provides a graphical representation of the dependencies and associations.

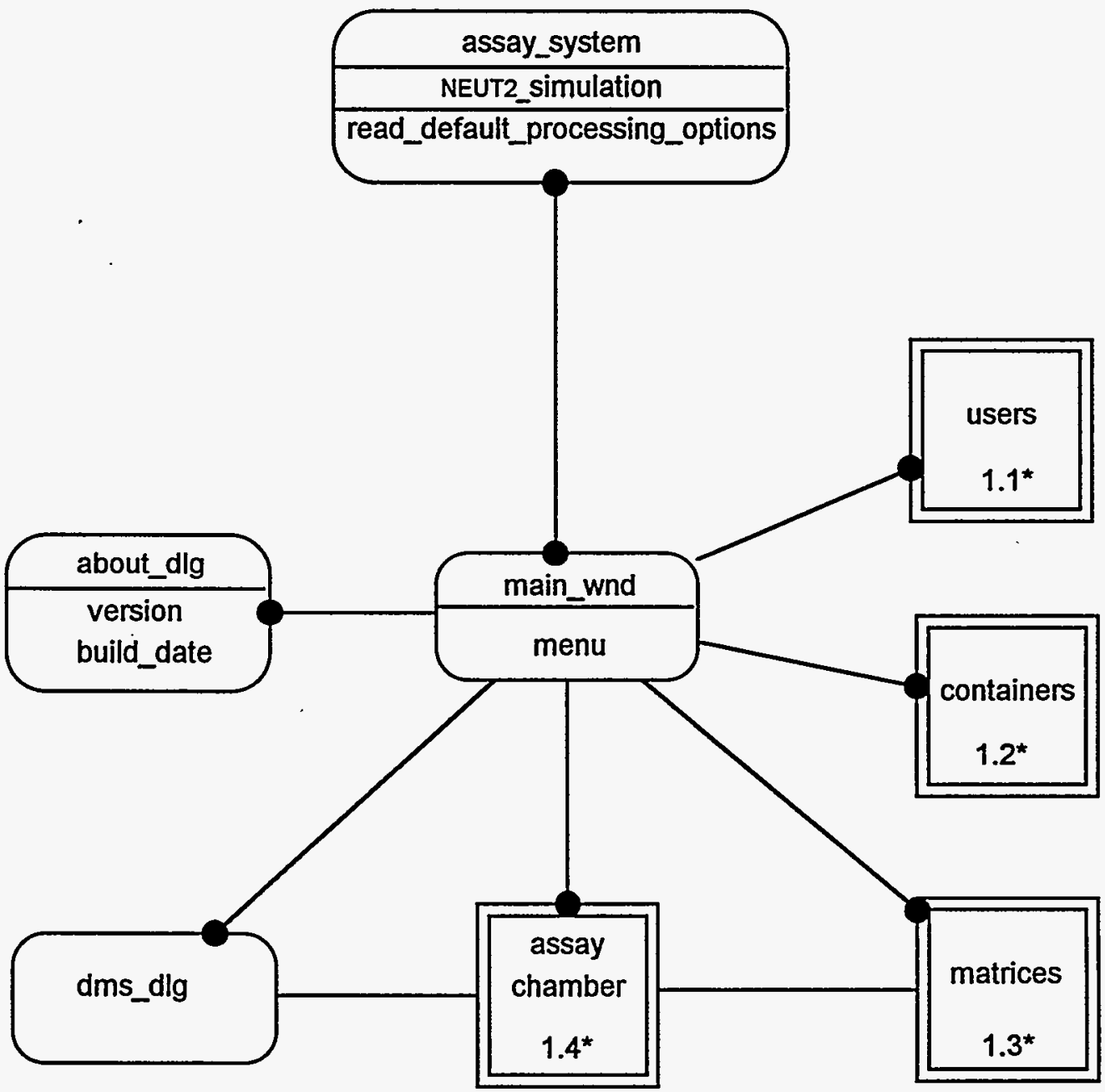

Figure 1. Assay system object model, level 1. 


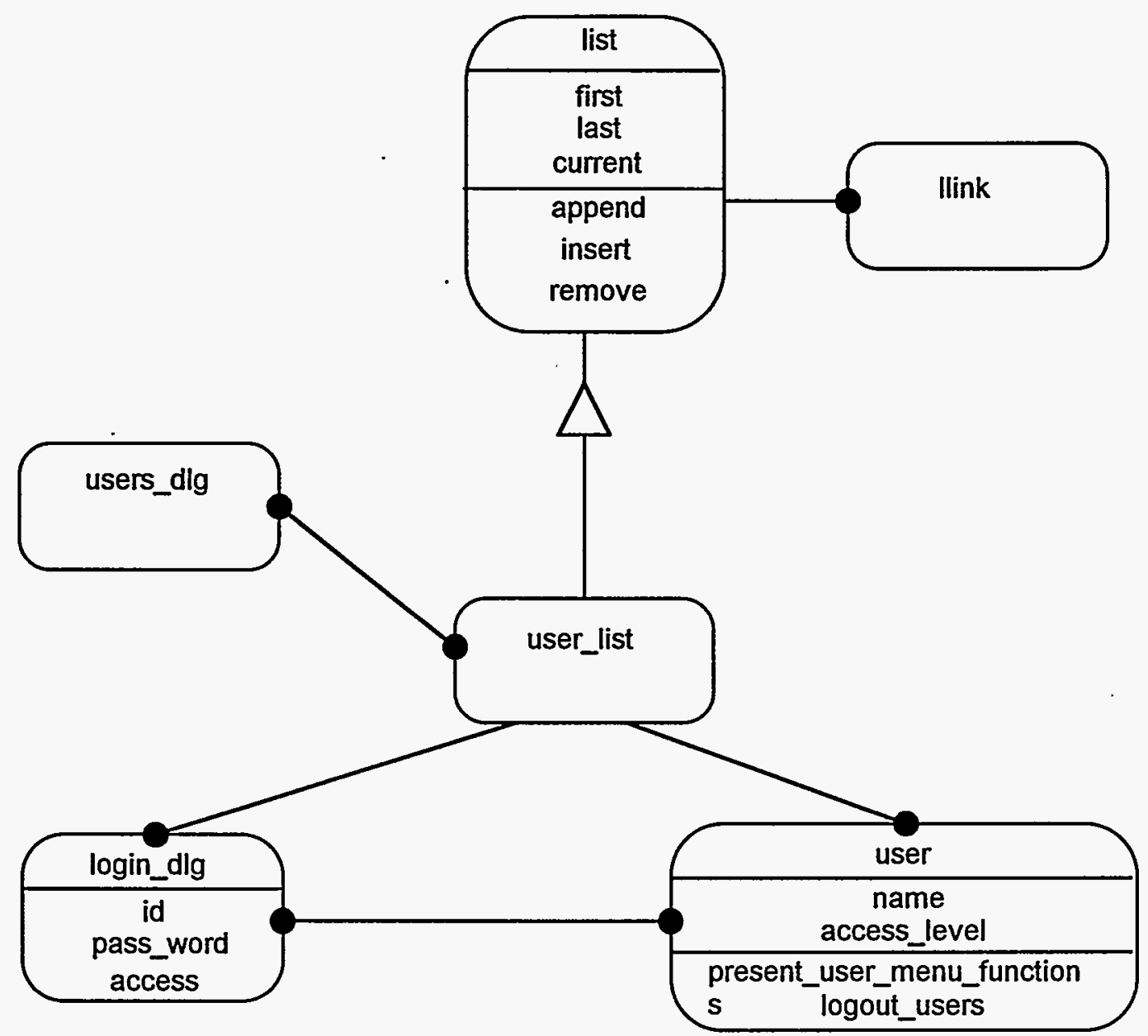

Figure 2. Assay system object model, level 1.1. 


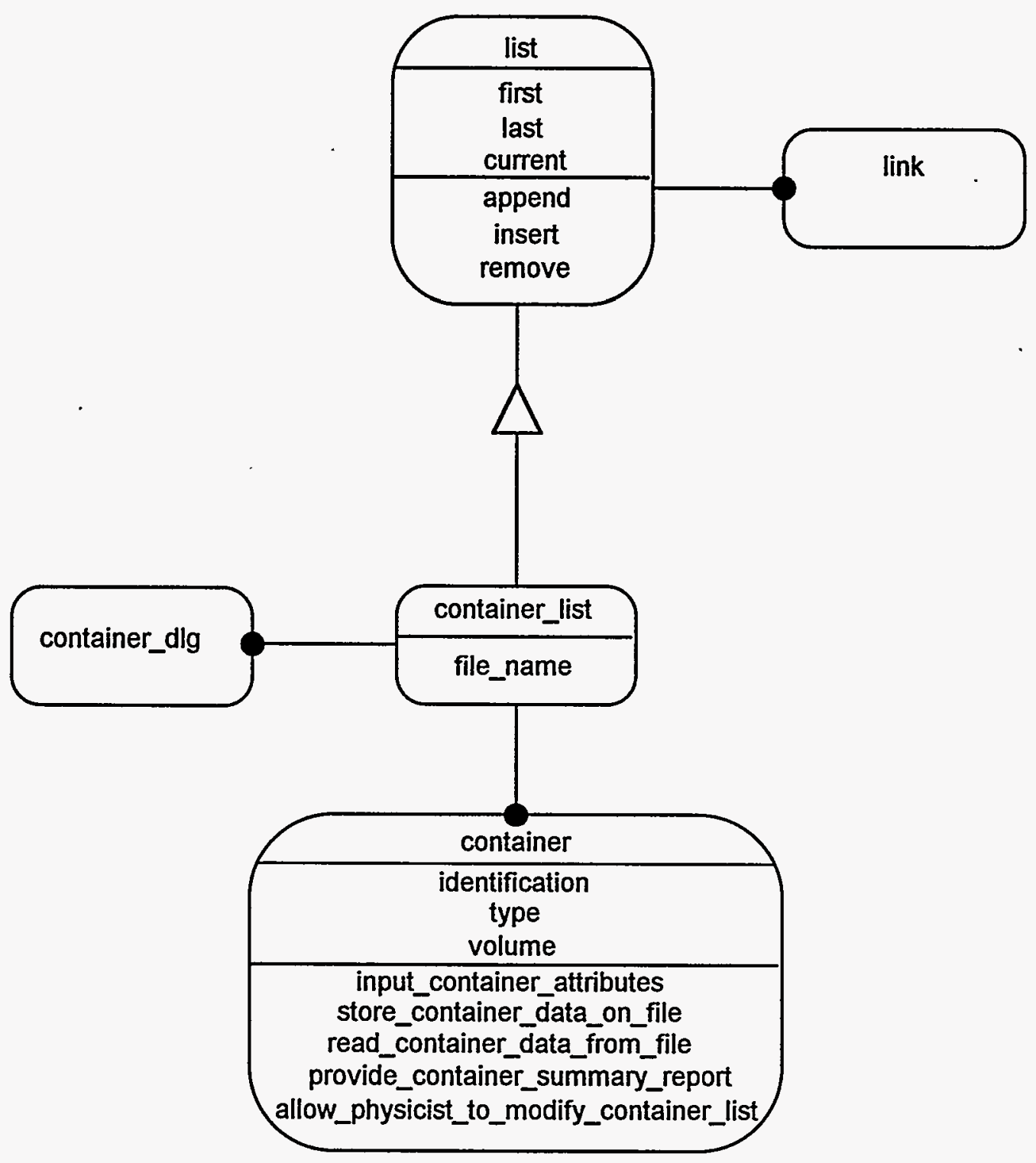

Figure 3. Assay system object model, level 1.2. 


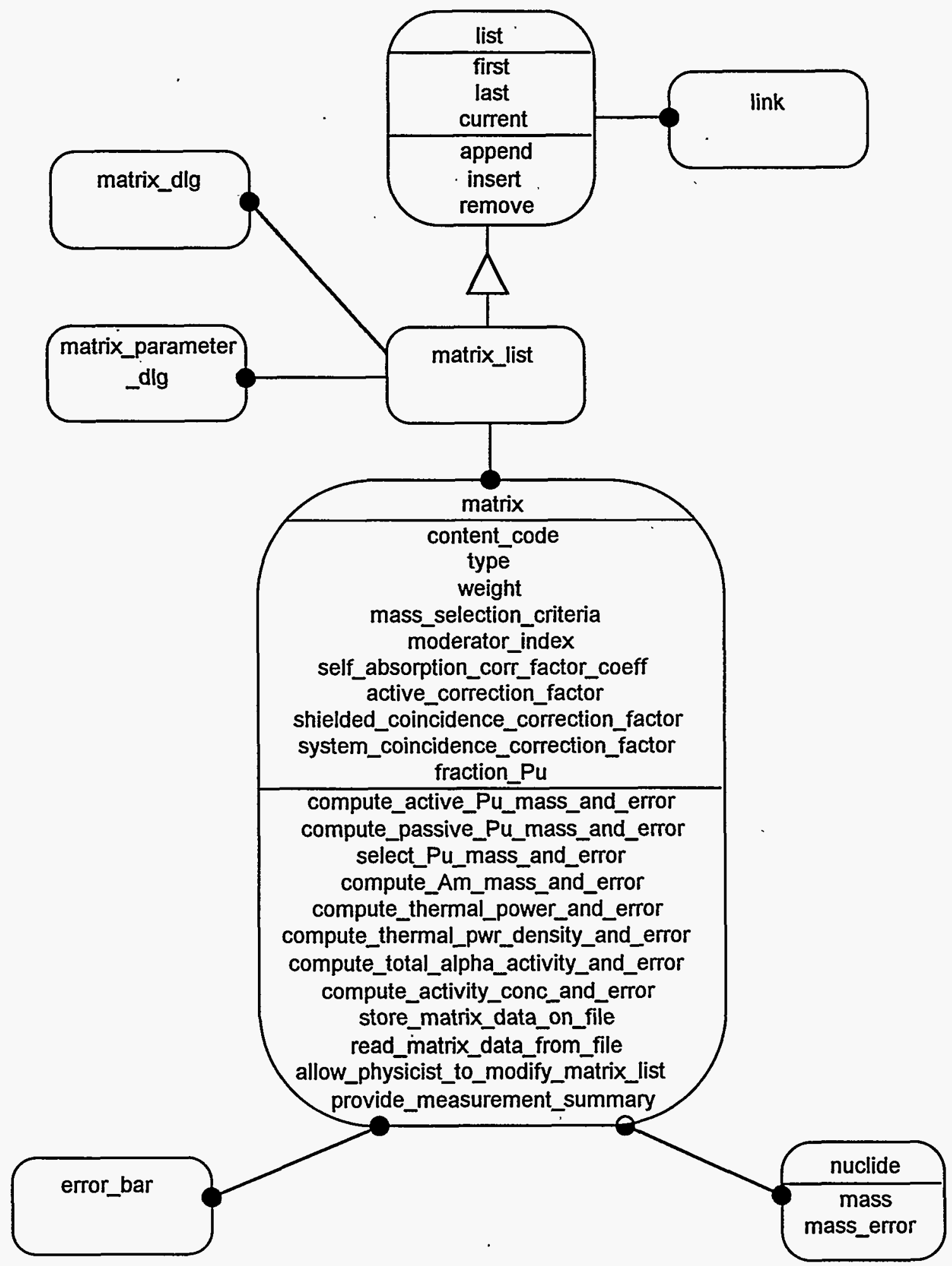

Figure 4. Assay system object model, level 1.3. 


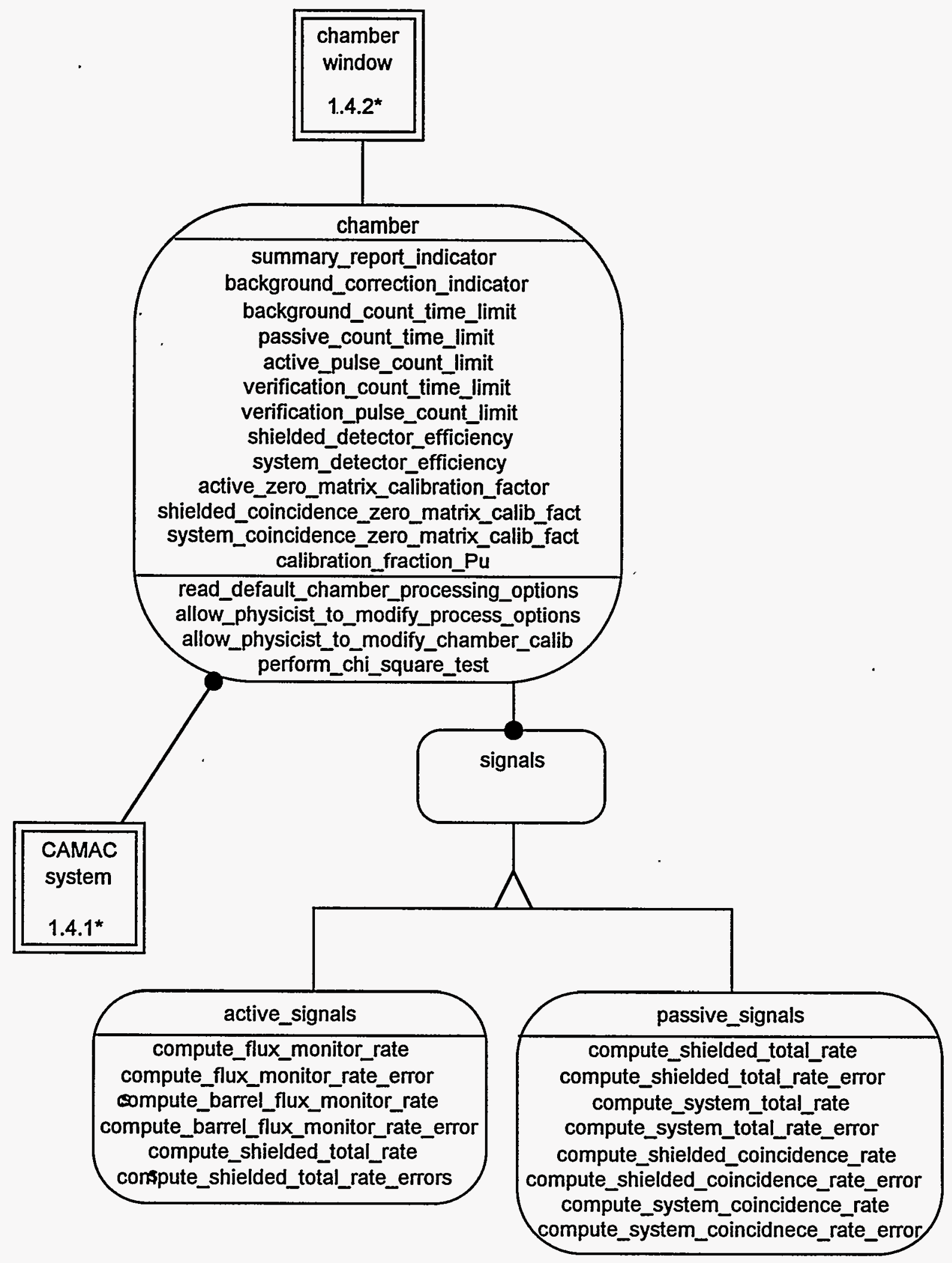

Figure 5. Assay system object model, level 1.4. 


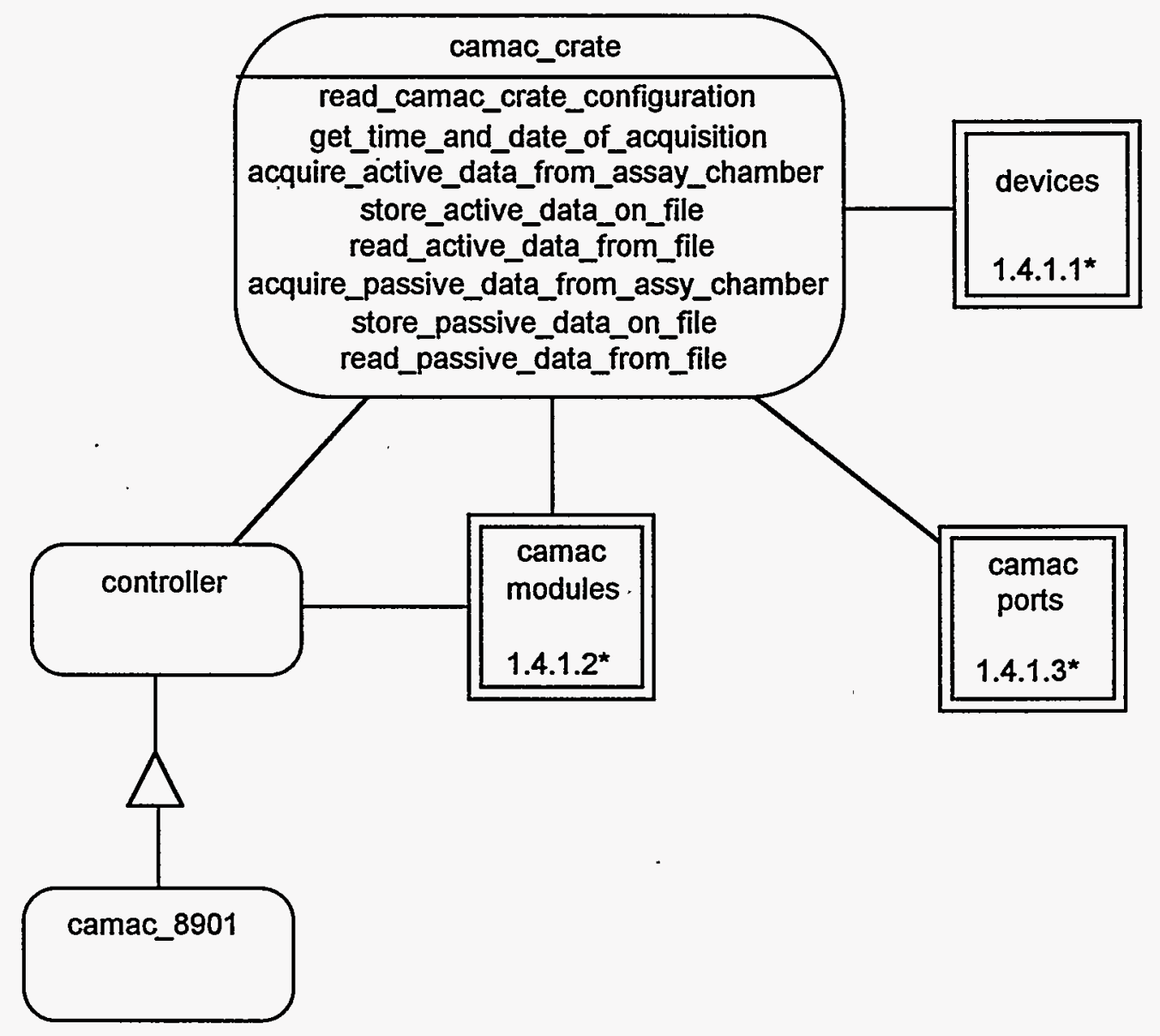

Figure 6. Assay system object model, level 1.4.1. 


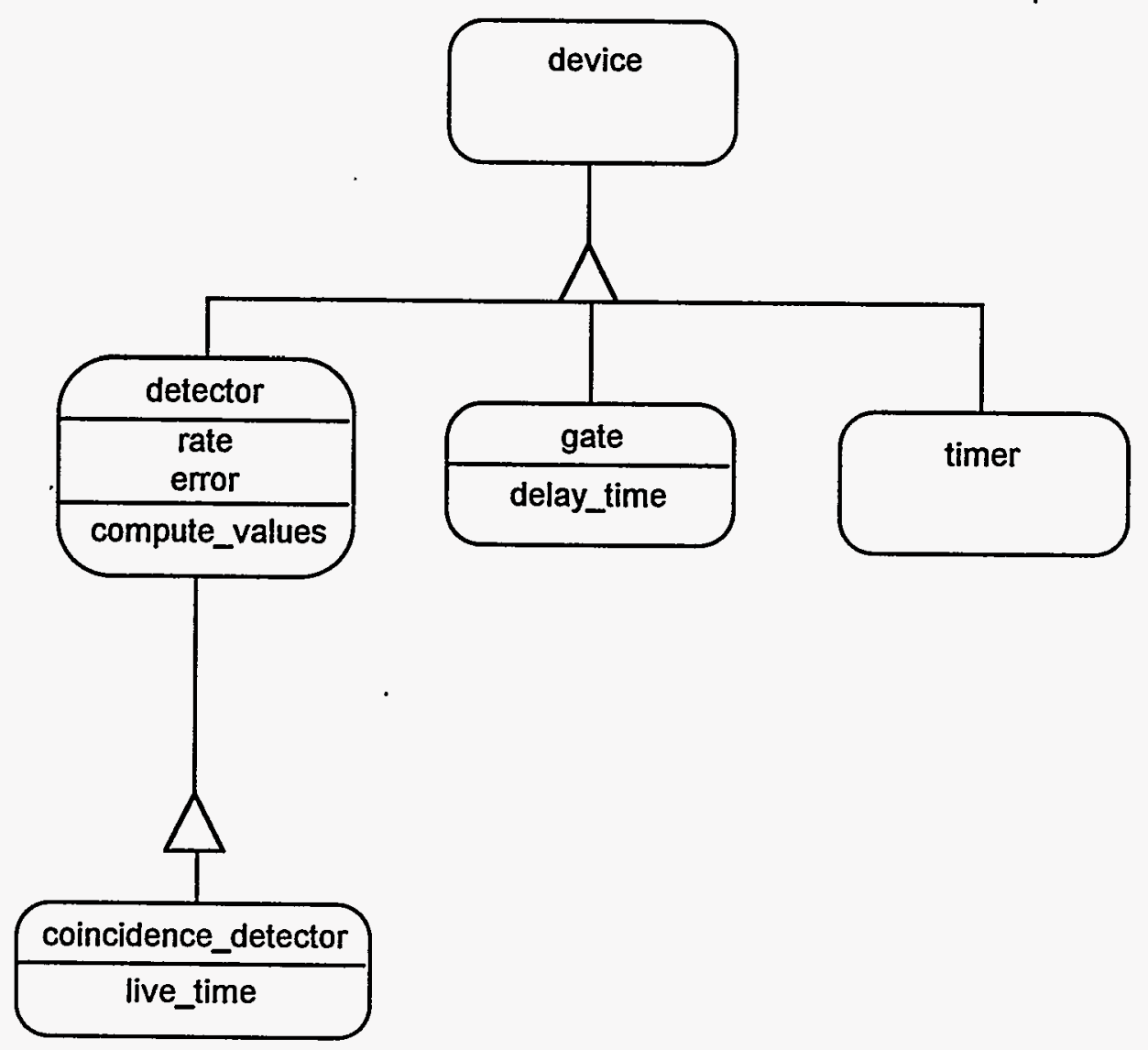

Figure 7. Assay system object model, level 1.4.1.1. 
INEL-96/0057

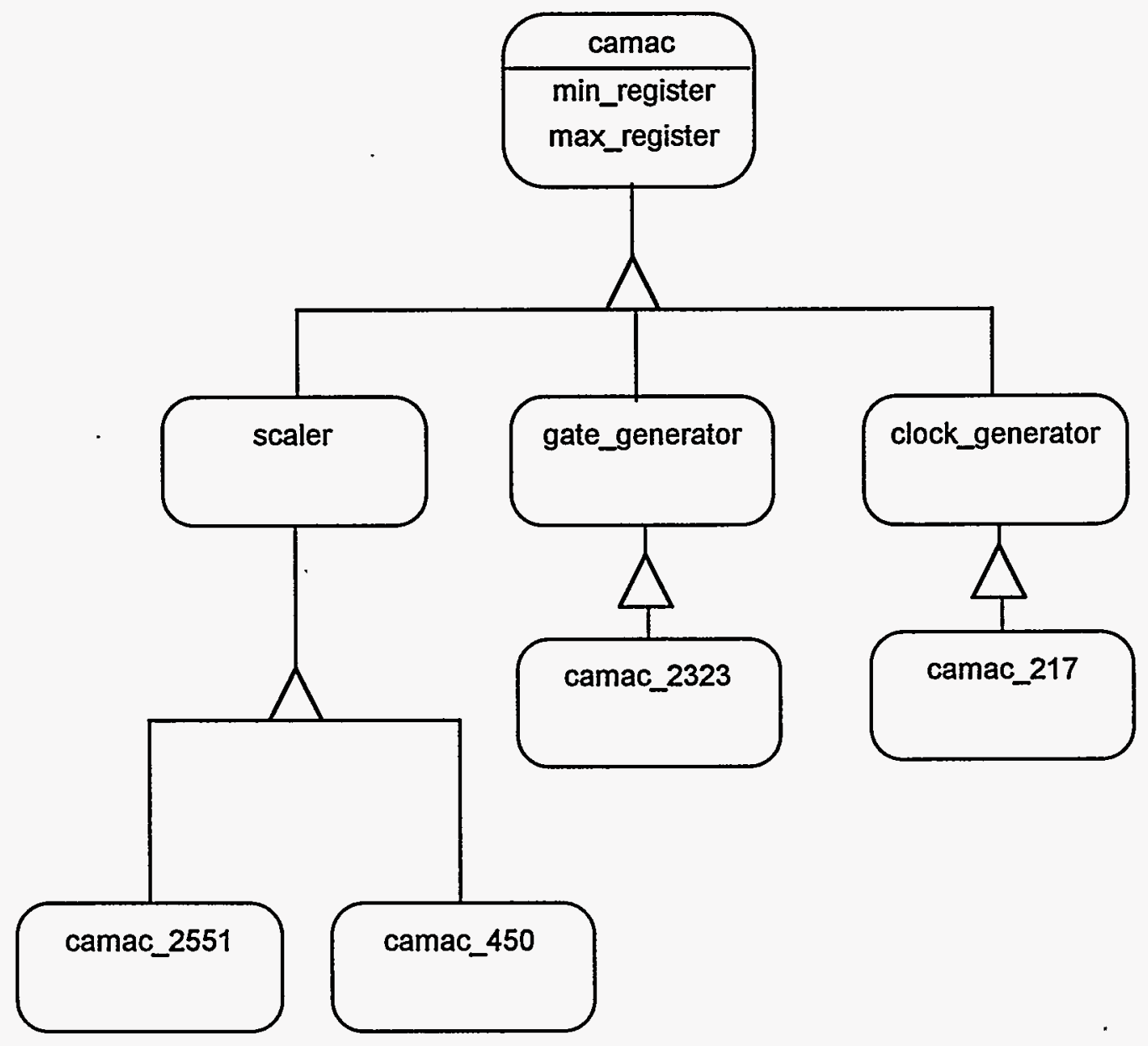

Figure 8. Assay system object model, level 1.4.1.2. 


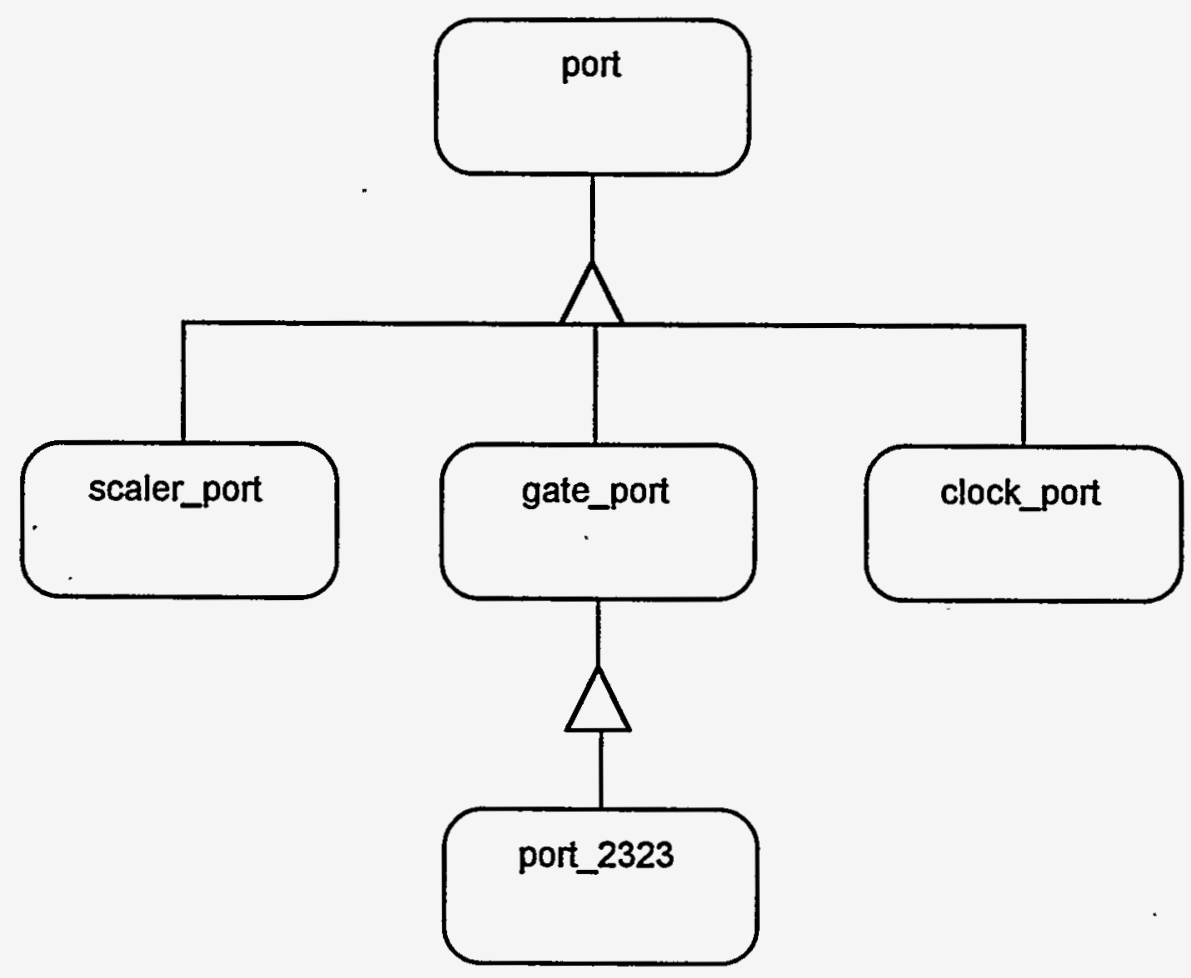

Figure 9. Assay system object model, level 1.4.1.3.

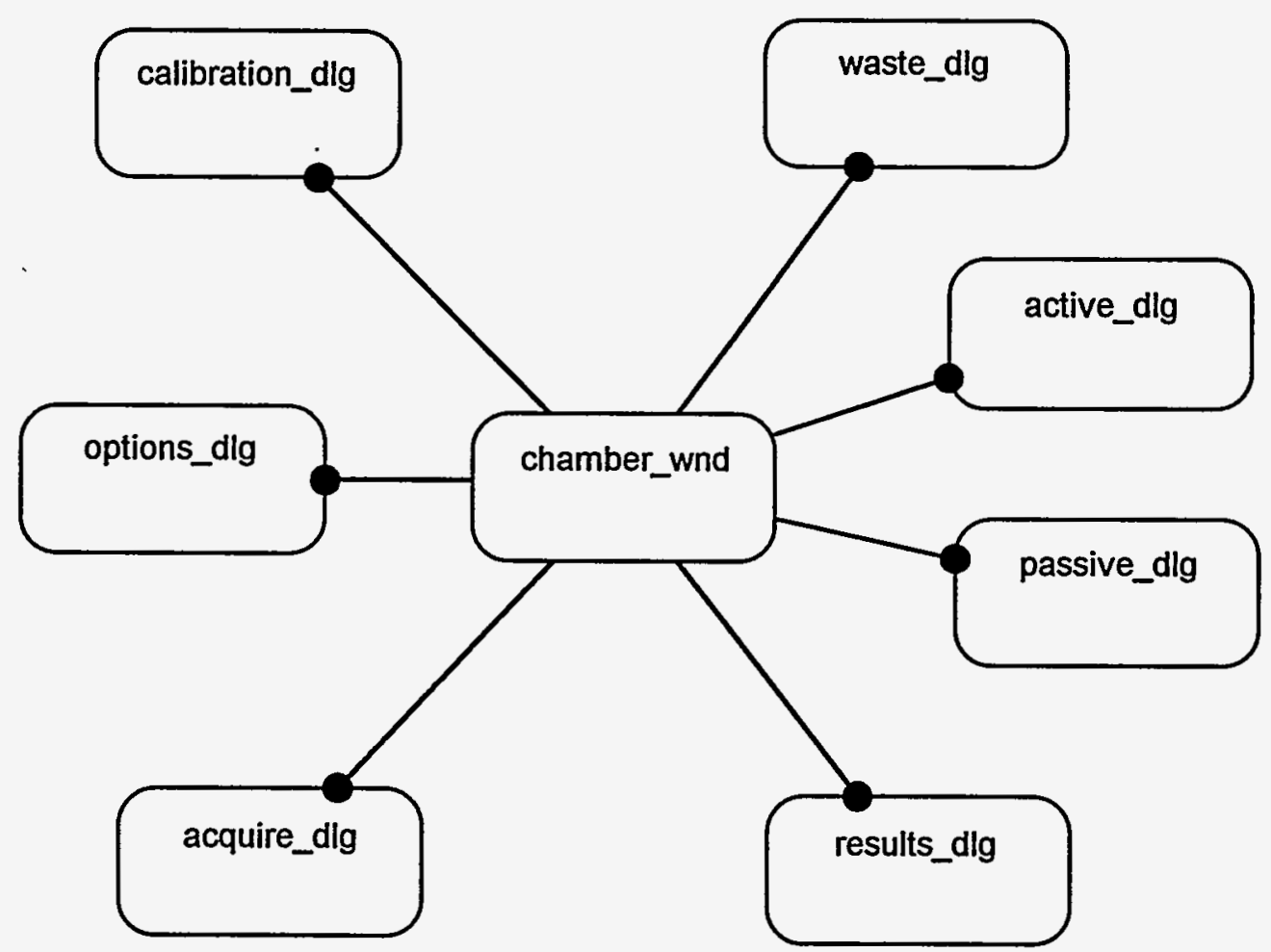

Figure 10. Assay system object model, level 1.4.2. 


\section{OBJECT DESCRIPTION}

This section provides a description, the decomposition, dependencies, and interfaces for each object. This information is most useful in conjunction with the object model in the previous section. Within this section objects are arranged alphabetically. A reference to a figure, where applicable, allows the reader to easily find the object in the object model.

\section{1 about_dlg}

\subsubsection{Description and Purpose}

The about dialog shows the version and the build date of the application (see Figure 1). The dialog is displayed when the user selects the menu item About.... It is dismissed by clicking on the Acknowledge button (see Figure 11).

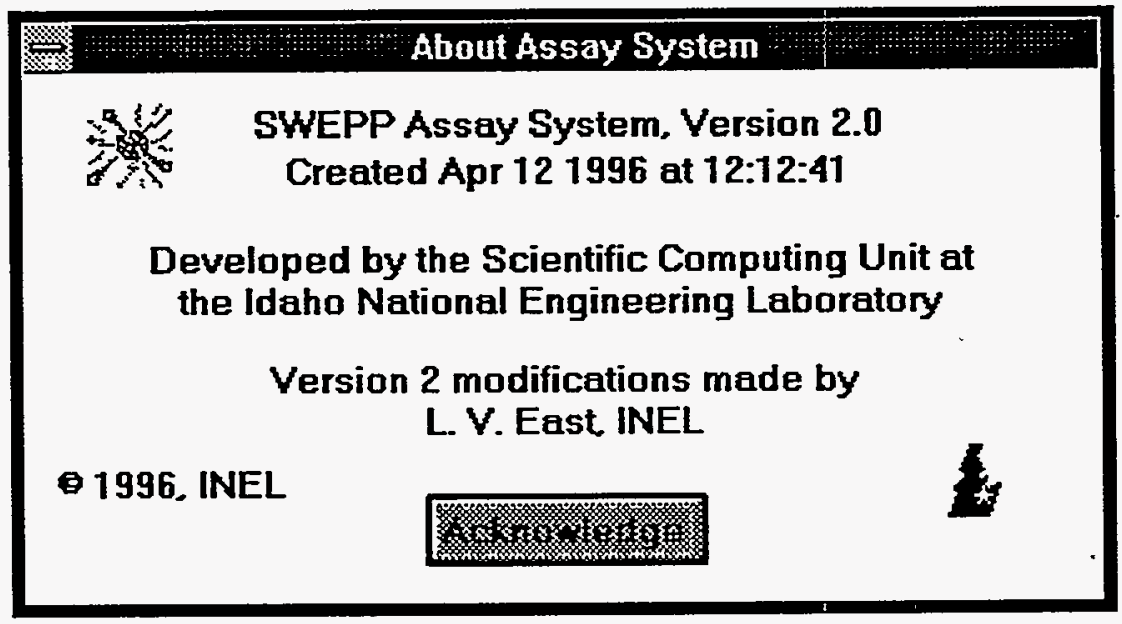

Figure 11. About dialog window.

\subsubsection{Decomposition}

This class is derived from CDialog, a class in the Microsoft Foundation Class (MFC) library. There are no subclasses.

\subsubsection{Dependencies}

It is created, displayed, and destroyed in a single function in the main window in response to the menu selection. The dialog receives the program identification and build date through the argument list of its creator. The about dialog may be displayed while a chamber window is active.

\subsubsection{Interfaces}

Files - none.

Windows - the about dialog box. 


\section{2 acquire_dlg}

\subsubsection{Description and Purpose}

The acquire dialog shows the status of a data acquisition (see Figure 10). The dialog title identifies the type of acquisition (Active, Passive Gross, Passive Background, or Chi-square). The required acquisition limit (pulse counts for active or time for passive) is displayed. The current acquisition count is displayed along with a graphic status bar that shows the percent of the acquisition completed. In addition, a message line provides additional status information or requests operator action, such as "Ensure that the assay chamber door is closed. Start the neutron pulse generator when ready." An operator may cancel the acquisition by clicking on the Cancel button (see Figure 12).

\subsubsection{Decomposition}

This class is derived from CDialog, a class in the MFC library. There are no subclasses.

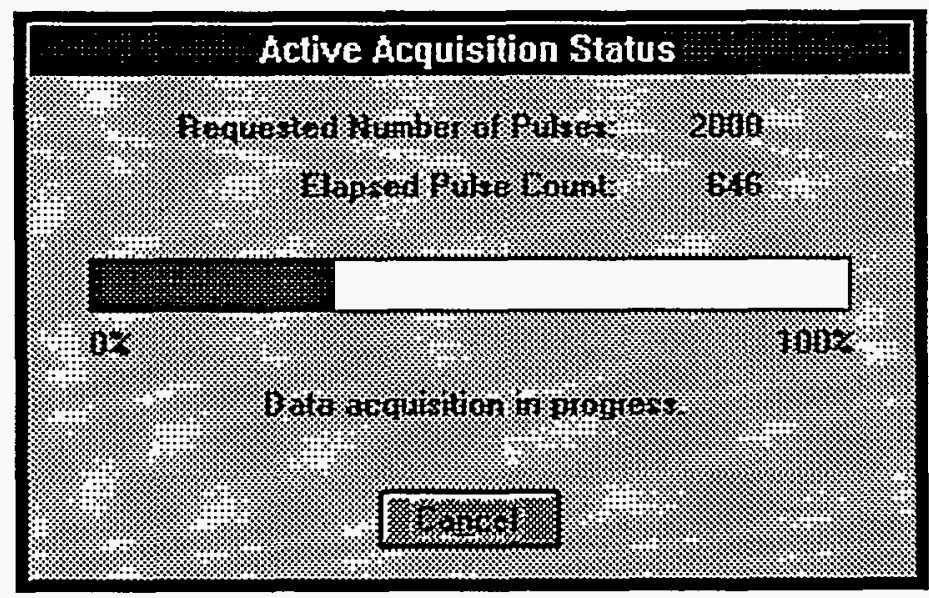

Figure 12. Acquire dialog window.

\subsubsection{Dependencies}

This object is created whenever one of the acquisition functions is invoked in the chamber window. The chamber window is responsible for ensuring that a CAMAC crate is ready to begin data acquisition. The acquire dialog creator function receives a pointer to a chamber through the argument list. The chamber pointer provides the dialog with the interface to get the name of the crate performing the acquisition and to get the limit value that determines when the acquisition is complete. The acquire dialog requests the chamber to start the acquisition, check the acquisition progress, and stop the acquisition.

\subsubsection{Interfaces}

Files - none.

Windows - the acquire dialog box. 


\section{3 active_dlg}

\subsubsection{Description}

The active dialog shows the counts and rates from an active data acquisition (see Figure 10). Both the gross and background counts are displayed for the shielded total, flux monitor, and the barrel flux monitor signals (see Figure 13). Rates for those same signals are displayed with an uncertainty ("error") of one standard deviation based on counting statistics [13].

\subsubsection{Decomposition}

This class is derived from CDialog, a class in MFC library. There are no subclasses.

\subsubsection{Dependencies}

This object is created by a chamber window when active data are acquired or read from a file. The active dialog creator function receives a pointer to an active signals object through the argument list. The active signals pointer provides the dialog with the interface to get the counts and rates that the dialog displays.

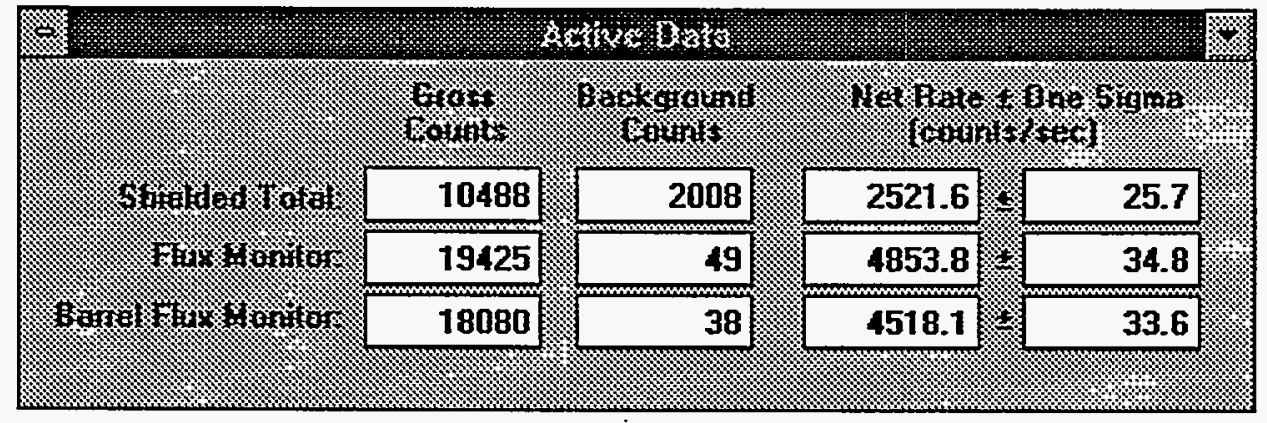

Figure 13. Active dialog (data) window.

\subsubsection{Interfaces}

Files - none.

Windows - the active dialog box.

\section{4 active_signals}

\subsubsection{Description}

The active signals class computes the net rates for the shielded total, flux monitor, and barrel flux monitor signals. (See Figure 5.)

\subsubsection{Decomposition}

This class is derived from the signals class (see below). There are no subclasses. 


\subsubsection{Dependencies}

An active signals object is created by a chamber. A pointer to the associated CAMAC crate that maintains the active data is passed as an argument to the active signals creator function. The CAMAC crate provides the interface to get the gross counts, background counts, and count rates for the active signals.

The gross count and rate are associated with the "early gate," and the background counts and rate are associated with the "late gate." These gates and the signal names are defined in the CAMAC crate configuration file. A change in the naming convention of the gates or signals will require a change in the active signals.

The active signals object is used by the active dialog to display the active data, and it is used by the matrix object in the data reduction.

\subsubsection{Interfaces}

Files - none.

Windows - none.

\section{5 assay_system}

\subsubsection{Description}

The assay system object provides the interface control for the SAS program. (See Figure 1.) This class is superordinate and addresses all the active and passive data assay requirements. Microsoft Windows runs the application as an instance of this object.

\subsubsection{Decomposition}

This class is derived from CWinApp in the MFC library. There are no subclasses.

This procedure creates the global assay system object. Once it is created, Microsoft Windows manages it.

\subsubsection{Dependencies}

The assay system defines the global character string for the program identification. This contains the system name and version number used for reports, data files, and the about dialog.

Microsoft Windows manages the windows interface when the application is created. The application is created when the user double clicks on the SWEPP Assay System icon. The assay system creates the main window and sends it the message to display the login dialog. Control is turned over to Microsoft Windows.

\subsubsection{Interfaces}

Files: none.

Windows: none. 


\section{6 calibration_dlg}

\subsubsection{Description}

The calibration dialog provides the user interface to change the chamber calibration parameters (see Figures 10) These include the detector efficiencies, the zero matrix calibration factors (active and passive shielded coincidence and passive system coincidence) and the distribution of plutonium to which the chamber is calibrated (see Figure 14).

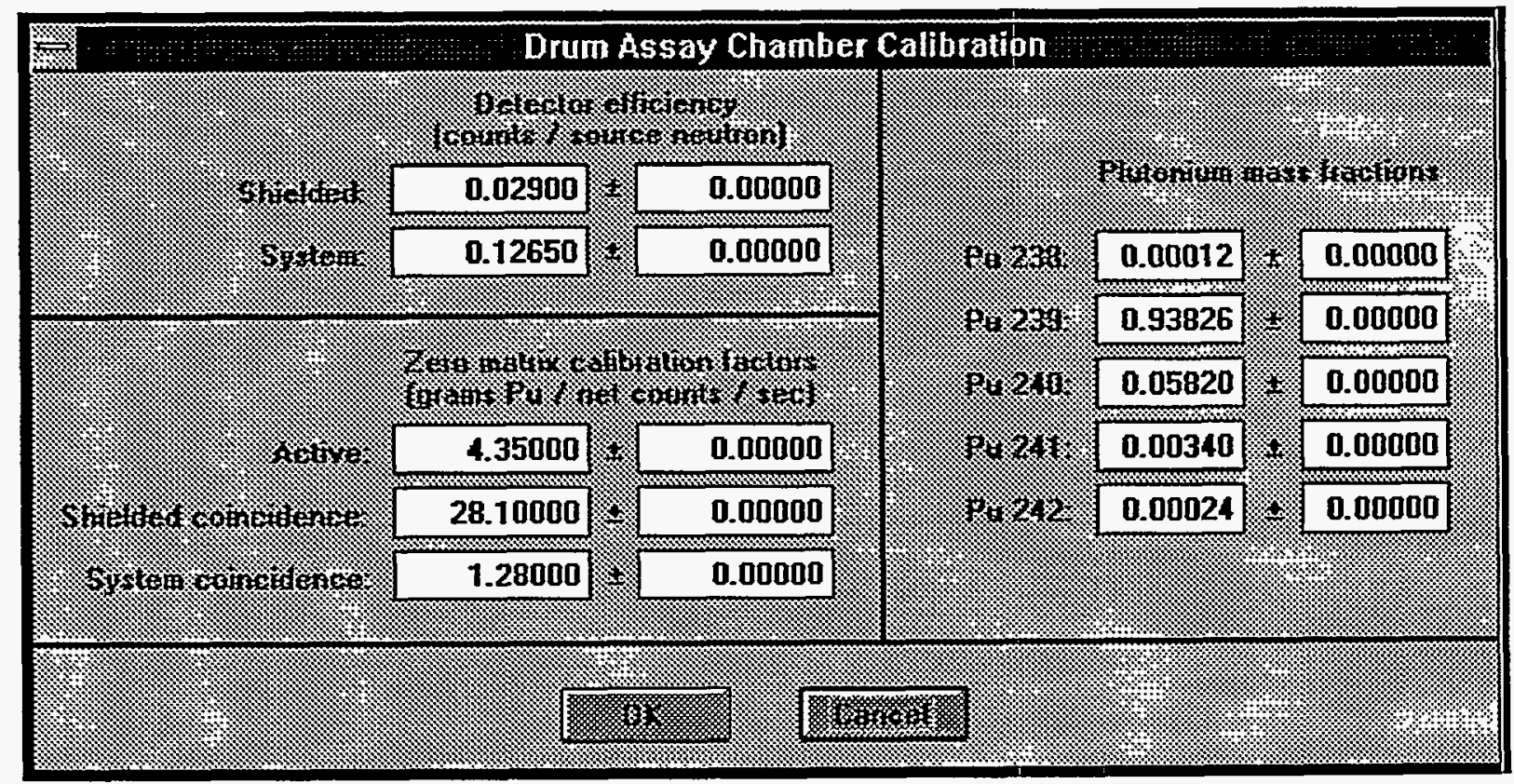

Figure 14. Calibration dialog window.

\subsubsection{Decomposition}

This class is derived from CDialog, a class in MFC library. There are no subclasses.

\subsubsection{Dependencies}

The calibration dialog is created by the chamber window in response to the menu selection Calibration... on the physicist's menu. A pointer to the chamber is passed as an argument to the creator function. The chamber provides the interface to the calibration parameters.

\subsubsection{Interfaces}

Files: none.

Windows: the calibration dialog. 


\section{7 camac}

\subsubsection{Description}

The camac class models a generic CAMAC module. (See Figure 8.) A module has a station number in the CAMAC crate, a type identifier, a model number (e.g., 217, 2323, 2551, 450, or 8901), minimum and maximum registers, and an array of associated ports (registers or addresses).

\subsubsection{Decomposition}

There is no superclass. Subclasses are clock generator, gate generator, and scaler.

\subsubsection{Dependencies}

A camac object is created by the CAMAC crate if the model of CAMAC module is not already in the defined set. Any camac object can interrogate its array of ports to locate a port with a specified name.

\subsubsection{Interfaces}

Files: none.

Windows: none.

\section{8 camac_217}

\subsubsection{Description}

The camac_217 class encapsulates the behavior of the Jorway 217 Gated Clock Generator. (See Figure 8.). This class has an array of clock ports.

\subsubsection{Decomposition}

This class is derived from the clock generator class. There are no subclasses.

\subsubsection{Dependencies}

A camac_217 object is created by the CAMAC crate. The camac_217 is associated with the crate controller that provides the interface to control the hardware module.

\section{8 .4 Interfaces}

Files: none.

Windows: none.

\section{9 camac_2323}

\subsubsection{Description}

The camac_2323 class encapsulates the behavior of the LeCroy 2323 Dual Gate and Delay Generator. (See Figure 8.) 
INEL-96/0057

\subsubsection{Decomposition}

This class is derived from the gate generator class: There are no subclasses.

\subsubsection{Dependencies}

A camac 2323 object is created by the CAMAC crate. The camac_2323 is associated with the crate controller that provides the interface to control the hardware board. This class has an array of gate ports specific to the 2323 module.

\subsubsection{Interfaces}

Files: none.

Windows: none.

\subsection{0 camac_2551}

\subsubsection{Description}

The camac_2551 class encapsulates the behavior of the LeCroy 255112 channel $100 \mathrm{MHz}$ Scaler. (See Figure 8.)

\subsubsection{Decomposition}

This class is derived from the scaler class. There are no subclasses.

\subsubsection{Dependencies}

A camac 2551 object is created by the CAMAC crate. The camac 2551 is associated with the crate controller that provides the interface to control the hardware module. This class has an array of scaler ports.

\subsubsection{Interfaces}

Files: none.

Windows: none.

\subsection{1 camac_450}

\subsubsection{Description}

The camac_450 class encapsulates the behavior of the DSP 251 QS-450, $100 \mathrm{MHz}$ Quad Scaler. (See Figure 8.)

\subsubsection{Decomposition}

This class is derived from the scaler class. There are no subclasses. 


\subsubsection{Dependencies}

A camac_450 object is created by the CAMAC crate. The camac_ 450 is associated with the crate controller that provides the interface to control the hardware module. This class has an array of scaler ports.

\subsubsection{Interfaces}

Files: none.

Windows: none.

\subsection{2 camac_8901}

\subsubsection{Description}

The camac_8901 encapsulates the behavior of the LeCroy 8901A GPIB interface. (See Figure 6.)

\subsubsection{Decomposition}

This class is derived from the controller class. There are no subclasses.

\subsubsection{Dependencies}

Each CAMAC module in a CAMAC crate is associated with a crate controller, such as the 8901 .

\subsubsection{Interfaces}

Files: none.

Windows: none.

\subsection{3 camac_crate}

\subsubsection{Description}

The camac_crate object encapsulates the behavior of the CAMAC crate system. (See Figure 6.) The camac_crate object manages the configuration of its CAMAC modules, ports, and devices. It initializes the active and passive assays and sets up and tests the CAMAC crate module and channel configuration. Camac_crate also reads and clears the scalers.

\subsubsection{Decomposition}

There is no superclass. There are no subclasses.

\subsubsection{Dependencies}

The chamber object creates camac_crate objects for the active data, the passive gross data, and the passive background data. The appropriate camac_crate object performs the initialization, starts an acquisition, checks the acquisition progress, and stops the acquisition. In addition, the camac_crate reads its configuration from a file and writes the configuration and acquired data to a file. 


\subsubsection{Interfaces}

Files: ACTCRATE.DAC, PASCRATE.DAC, ACTCRATE.CAC, PASCRATE.CAC, and data files for acquisition data.

Windows: none.

\subsection{4 chamber}

\subsubsection{Description}

The chamber class models an assay chamber. (See Figure 5.) Physically, the two assay chambers are the drum assay chamber and the crate assay chamber. Logical chambers may be used for calibration and test purposes. .

\subsubsection{Decomposition}

There is no superclass. There are no subclasses.

\subsubsection{Dependencies}

A chamber object is associated with its corresponding chamber window. A chamber has public pointers to active signals and passive signals. A chamber also is associated with the CAMAC crate. Physically, there is only one CAMAC crate modeled logically as three CAMAC crate objects, one for active data, one for passive data, and one for passive background. The chamber provides the interface for the chamber window to start data acquisition, check acquisition progress, and stop data acquisition for the each type of data.

The chamber allows the calibration dialog friend class access to its attributes. The calibration dialog provides the user interface to change the calibration data (detector efficiencies, zero matrix calibration factors, and plutonium mass distribution to which the chamber is calibrated).

\subsubsection{Interfaces}

Files: CALIB.DAC and CALIB.CAC.

Windows: none.

\subsection{5 chamber_wnd}

\subsubsection{Description}

The chamber window class provides the user interface to the assay chamber operations and the file interface to the operating system (see Figure 10). Windows that display assay data and results are child windows of the chamber window (see Figure 15). 


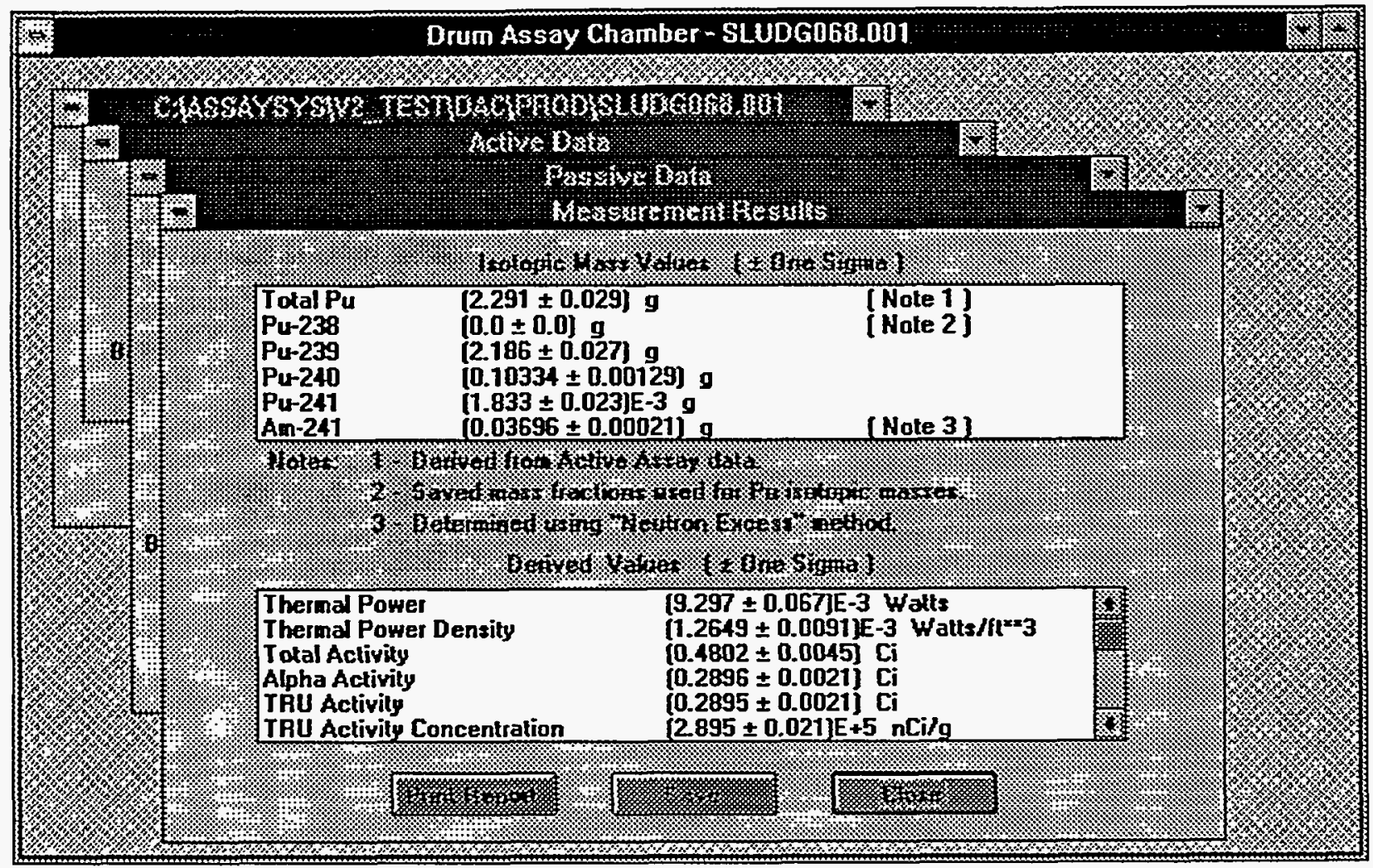

Figure 15. Assay chamber window and child windows.

\subsubsection{Decomposition}

This class is derived from CMDIChildWnd, a class in the MFC library.

\subsubsection{Dependencies}

The chamber window is created by the main window. Each chamber window has its own configuration file. The chamber window is associated with a chamber. The process options dialog provides the interface to set the pulse count limits and the count time limits for the active and passive data acquisitions respectively.

The chamber window is also associated with the dms dialog, when the dms interface is active.

\subsubsection{Interfaces}

Files: DAC.INI, OPTIONS.DAC, CHISQ.DAC, CAC.INI, OPTIONS.CAC, and CHISQ.CAC.

Windows: the chamber window.

\subsection{6 clock_generator}

\subsubsection{Description}

The clock_generator object models a generic CAMAC clock generator board. (See Figure 8.) 
INEL-96/0057

\subsubsection{Decomposition}

This class is derived from the camac class. It has camac_217 as a subclass.

\subsubsection{Dependencies}

The clock generator is associated with an array of clock ports. This provides the interface to get and set the clock rate for individual ports.

\subsubsection{Interfaces}

Files: none.

Windows: none.

\subsection{7 clock_port}

\subsubsection{Description}

The clock port class models a port (register or address) on a CAMAC clock generator. (See Figure 9.) A clock port generates clock pulses at a prescribed rate.

\subsubsection{Decomposition}

This class is derived from the port class. There are no subclasses.

\subsubsection{Dependencies}

The clock port is created by a camac_217 object, which also sets the clock rates for designated clock ports. The clock rate is retrieved by a timer object.

\subsubsection{Interfaces}

Files: none.

Windows: none.

\subsection{8 coincidence_detector}

\subsubsection{Description}

The coincidence_detector class computes the coincidence rate, correcting the rate for accidental counts. (See Figure 7.)

\subsubsection{Decomposition}

This class is derived from the detector class. There are no subclasses.

\subsubsection{Dependencies}

A coincidence_detector is associated with a scaler_port, a detector that has the same name as the coincidence_detector, a gate, and a timer. It is created by the camac_crate object when specific devices are 
associated with the scaler ports. The gate and the timer provide times that are used together with the count from the scaler port to compute a rate. Access to the rate is provided through the camac_crate.

\subsubsection{Interfaces}

Files: none.

Windows: none.

\subsection{9 container}

\subsubsection{Description}

The container object models the behavior of a generic container process at the assay station. (See Figure 3.) A container has an identification, such as a Rocky Flats ID, a type description, such as 55-gallon drum, and a volume in litters.

\subsubsection{Decomposition}

There is no superclass. There are no subclasses.

\subsubsection{Dependencies}

A container is associated with a matrix. The container provides the interface to get the volume used in data reduction. The container produces a report when requested.

\subsubsection{Interfaces}

Files: none.

Windows: none.

Reports: summary report.

\subsection{0 container_dlg}

\subsubsection{Description}

The container dialog class provides the user interface to modify the list of container types available for processing. (See Figures 3 and 16.)

\subsubsection{Decomposition}

This class is derived from CDialog, a class in the MFC library.

\subsubsection{Dependencies}

This class is created by main window when the menu item Container List... is selected. The container dialog is associated with the container list. 


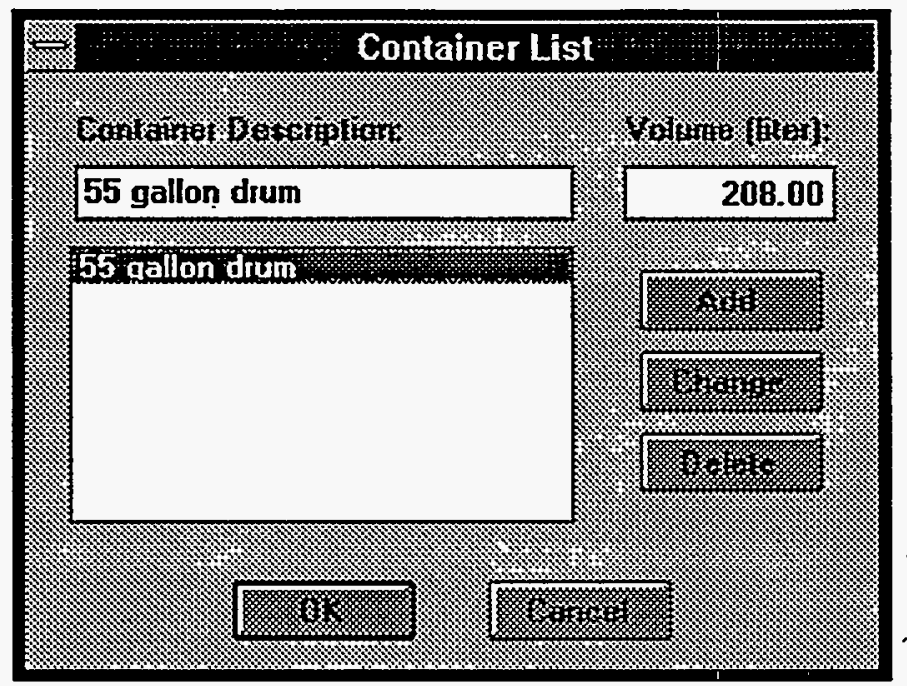

Figure 16. Container dialog window.

\subsubsection{Interfaces}

Files: none.

Windows: the container dialog.

\subsection{1 container_list}

\subsubsection{Description}

The container_list class models the list of containers used at the assay station. (See Figure 3.)

\subsubsection{Decomposition}

This class is derived from the list class. There are no subclasses.

\subsubsection{Dependencies}

The container list is created by the main window. It is used in the container window to select a waste container type for processing. It is also used in the container dialog to modify the list of available containers.

\subsubsection{Interfaces}

Files: none.

Windows: none. 


\subsection{2 controller}

\subsubsection{Description}

The controller object encapsulates all the functionality for the CAMAC crate controller, regardless of the actual controller implementation. (See Figure 6.) This functionality includes transmitting an instruction to a controller and getting a response from a controller.

\subsubsection{Decomposition}

There is no superclass. There is one subclass, the camac_8901.

\subsubsection{Dependencies}

A controller is associated with each instance of a CAMAC module.

\subsubsection{Interfaces}

Files: none.

Windows: none.

\subsection{3 delay_dlg}

\subsubsection{Description}

A delay_dlg object is used to provide a delay between active and passive data acquisitions and indicate the time remaining to the user. The delay_dlg class was added in Version 2.0.

\subsubsection{Decomposition}

This class is derived from CDialog, a class in the MFC library. There are no subclasses.

\subsubsection{Dependencies}

A delay_dlg is created by a chamber object whenever passive data collection follows an active data collection and the delay time specified in the chamber configuration file is greater than zero.

\subsubsection{Interfaces}

Files: none.

Windows: Delay dialog window (Figure 17). 


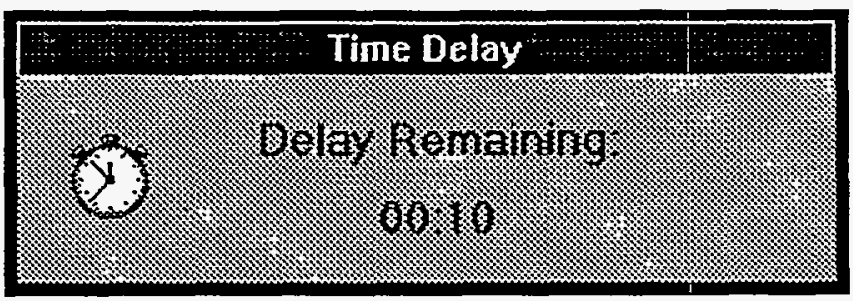

Figure 17. Delay dialog window.

\subsection{4 detector}

\subsubsection{Description}

The detector object models a neutron detector or group of detectors in the assay chamber. (See Figure 7.)

\subsubsection{Decomposition}

This object is derived from device. There is one subclasses, coincidence_detector.

\subsubsection{Dependencies}

A detector is associated with either a timer or a gate or both. The timer or gate provide an elapsed time, along with the counts from the associated scaler port, that the detector object uses to compute its rate.

\subsubsection{Interfaces}

Files: none.

Windows: none.

\subsection{5 device}

\subsubsection{Description}

The device object models a physical or logical device. (See Figure 7.) Derived classes, such as gate, timer, detector, or coincidence_detector, model behavior specific for each device. The device is associated with a scaler port.

\subsubsection{Decomposition}

This is no superclass. Subclasses are detector, gate, and timer.

\subsubsection{Dependencies}

The device is associated with a scaler port through the device list. 


\subsubsection{Interfaces}

Files: none.

Windows: none.

\subsection{6 device_list}

\subsubsection{Description}

The device_list class models a list of logical or physical devices.

\subsubsection{Decomposition}

This class is derived from the list class. There are no subclasses.

\subsubsection{Dependencies}

One or more devices are associated with a scaler port through the device list. The scaler port may interrogate the list through the get_first, get_next, and find functions of the device list.

\subsubsection{Interfaces}

Files: none.

Windows: none.

\subsection{7 dms_dlg}

\subsubsection{Description}

The dms_dlg (dialog) class defines the interface with the DMS "remote" computer. (See Figure 1.) It opens a communications port, receives a request from the DMS, sends results to the DMS, and acknowledges that the DMS has received data. The DMS dialog window is shown in Figure 17.

\subsubsection{Decomposition}

This class is derived from CDialog, a class in the MFC library. There are no subclasses.

\subsubsection{Dependencies}

A single instance of dms dlg is created by the main window, which it then associates with the chamber windows. The dms_dlg object passes requests for assays or recalculations to the main window. The main window determines the appropriate assay chamber to perform the request and sends the request to the chamber window.

The chamber window then asks the dms dlg object to provide the details of the request, e.g., the container identification and the file identification. When processing is complete, the chamber window sends the dms_dlg object a message requesting that the assay results be forward to the DMS computer. 
INEL-96/0057

\subsubsection{Interfaces}

Files: none.

Windows: the dms dialog.

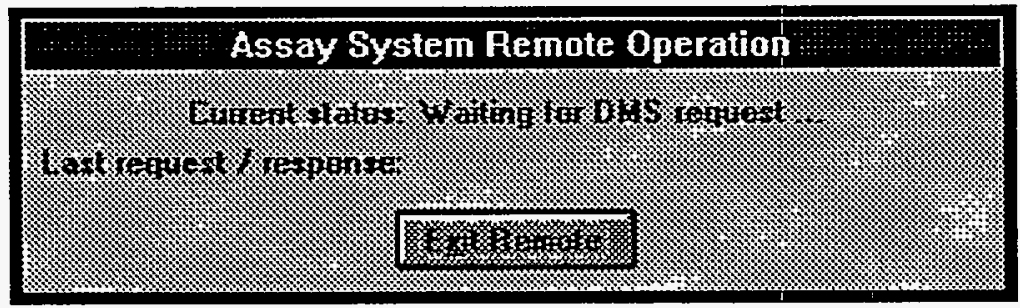

Figure 18. DMS dialog window.

\subsection{8 error_bar}

\subsubsection{Description}

The error_bar object class models an "error bar", that is, a value plus 'or minus one standard deviation uncertainty. (See Figure 4.) Overloaded operators for addition, subtraction, multiplication and division for error_bar and error_bar in conjunction with float variables are defined to propagate the error through the computations. Overload operators for comparison operations were added in Version 2; these operators take into account the uncertainties associated with error_bar values at the 95 percent confidence level when performing comparisons between error_bar values; see Reference [14], Section 2-3.

\subsubsection{Decomposition}

There is no superclass. There are no subclasses.

\subsubsection{Dependencies}

This class is used by the matrix class in performing the data reduction. The chamber calibration parameters, mass fractions and mass ratios are also defined as error bars.

\subsubsection{Interfaces}

Files: none.

Windows: none.

\subsection{9 gate}

\subsubsection{Description}

The gate class models the functionality of a CAMAC gate module, which is a logical device. (See Figure 7.) A gate defines a window in time wherein certain events are counted. 


\subsubsection{Decomposition}

This class is derived from the device class.

\subsubsection{Dependencies}

A gate is created by the camac_crate object when the configuration information is read. A gate is associated with a scaler port and a gate port. These provide the interface for a gate to get the count of the number of gates and the gate width from which the gate computes its delay time and its gate time.

\subsubsection{Interfaces}

Files: none.

Windows: none.

\subsection{0 gate_generator}

\subsubsection{Description}

The gate generator class models the generic functionality of a CAMAC gate generator module. (See Figure 8.)

\subsubsection{Decomposition}

This class is derived from the camac class. There is one subclass, the camac_2323.

\subsubsection{Dependencies}

A gate_generator object has gate ports associated with it. The gate ports retain the information necessary to program the gate generator.

\subsubsection{Interfaces}

Files: none.

Windows: none.

\subsection{1 gate_port}

\subsubsection{Description}

The gate_port class models a port or address on a CAMAC crate gate generator. (See Figure 9.)

\subsubsection{Decomposition}

This class is derived from the port class. There is one subclass, the port_2323.

\subsubsection{Dependencies}

A gate_port is associated with a gate_generator. 
INEL-96/0057

\subsubsection{Interfaces}

Files: none.

Windows: none.

\subsection{2 hstreambuf}

\subsubsection{Description}

The hstreambuf models the Hewlett-Packard LaserJet printer as a stream buffer.

\subsubsection{Decomposition}

This class is derived from filebuf, a class in the iostream library provided with the $\mathrm{C}++$ language.

\subsubsection{Dependencies}

This class is defined whenever a user requests a file to be printed or a report is generated. It is associated with the print file.

\subsubsection{Interfaces}

Files: none.

Windows: none.

\subsection{3 itstream}

\subsubsection{Description}

The itstream class models an input token stream.

\subsubsection{Decomposition}

This class is derived from ifstream, a class in the iostream library provided with the $\mathrm{C}++$ language.

\subsubsection{Dependencies}

The itstream class is created by the main window or a chamber window whenever a file must be read as an input token stream.

\subsubsection{Interfaces}

Files: none.

Windows: none.

\subsection{4 link}

\subsubsection{Description}

The link class models a link used in constructing a linked list. (See Figures 2, 3, and 4.) 


\subsubsection{Decomposition}

There is no superclass. There are no subclasses.

\subsubsection{Dependencies}

The link class is created by a list.

\subsubsection{Interfaces}

Files: none.

Windows: none.

\subsection{5 list}

\subsubsection{Description}

The list class models a generic linked list. This provides the facility to add, remove, insert, replace, and find items in a list. (See Figures 2, 3, and 4.)

\subsubsection{Decomposition}

There are no superclasses. Subclasses are container_list, device_list, matrix_list, and user_list.

\subsubsection{Dependencies}

The list class is not intended to be created itself.

\subsubsection{Interfaces}

Files: none.

Windows: none.

\subsection{6 login_dlg}

\subsubsection{Description}

The login dialog class provides the user interface to log into the system. (See Figure 2.) The login dialog validates the user against the user list.

\subsubsection{Decomposition}

This class is derived from CDialog, a class in the MFC library. There are no subclasses.

\subsubsection{Dependencies}

A login dialog is created by the main window when the menu item Login... is selected. 
INEL-96/0057

\subsubsection{Interfaces}

Files: none.

Windows: the login dialog (see Figure 19).

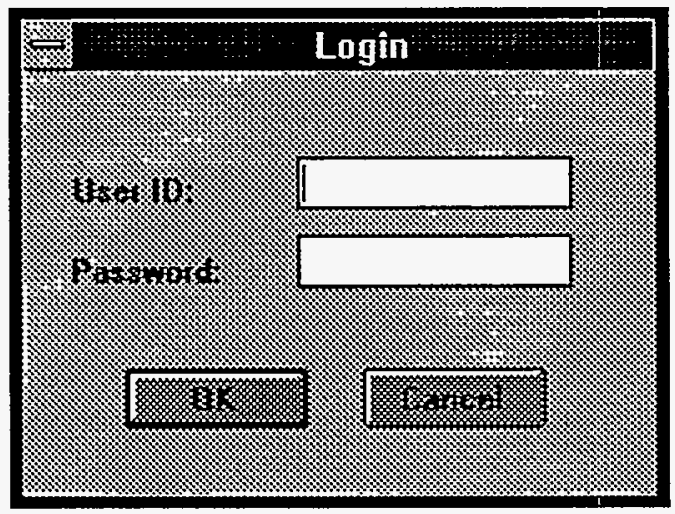

Figure 19. User login dialog window.

\subsection{7 main_wnd}

\subsubsection{Description}

The main wnd class provides the overall management of the user interface and the operating system interface. (See Figure 1.) There is only one main_wnd object per program instance, and it creates and manages the main program window.

\subsubsection{Decomposition}

This class is derived from CMDIFrameWnd, a class in the MFC library. There are no subclasses.

\subsubsection{Dependencies}

This class creates the user_list, container_list, and the matrix_list objects as global resources. The main_wnd object creates chamber windows as child windows, as specified in the configuration (see Figure 19).

The main window is associated with the container dialog, the matrix dialog, and the user dialog. Also, the main window provides interface to the about dialog.

\subsubsection{Interfaces}

Files: AS.INI, the log file, the error file.

Windows: the main window (Figure 20). 


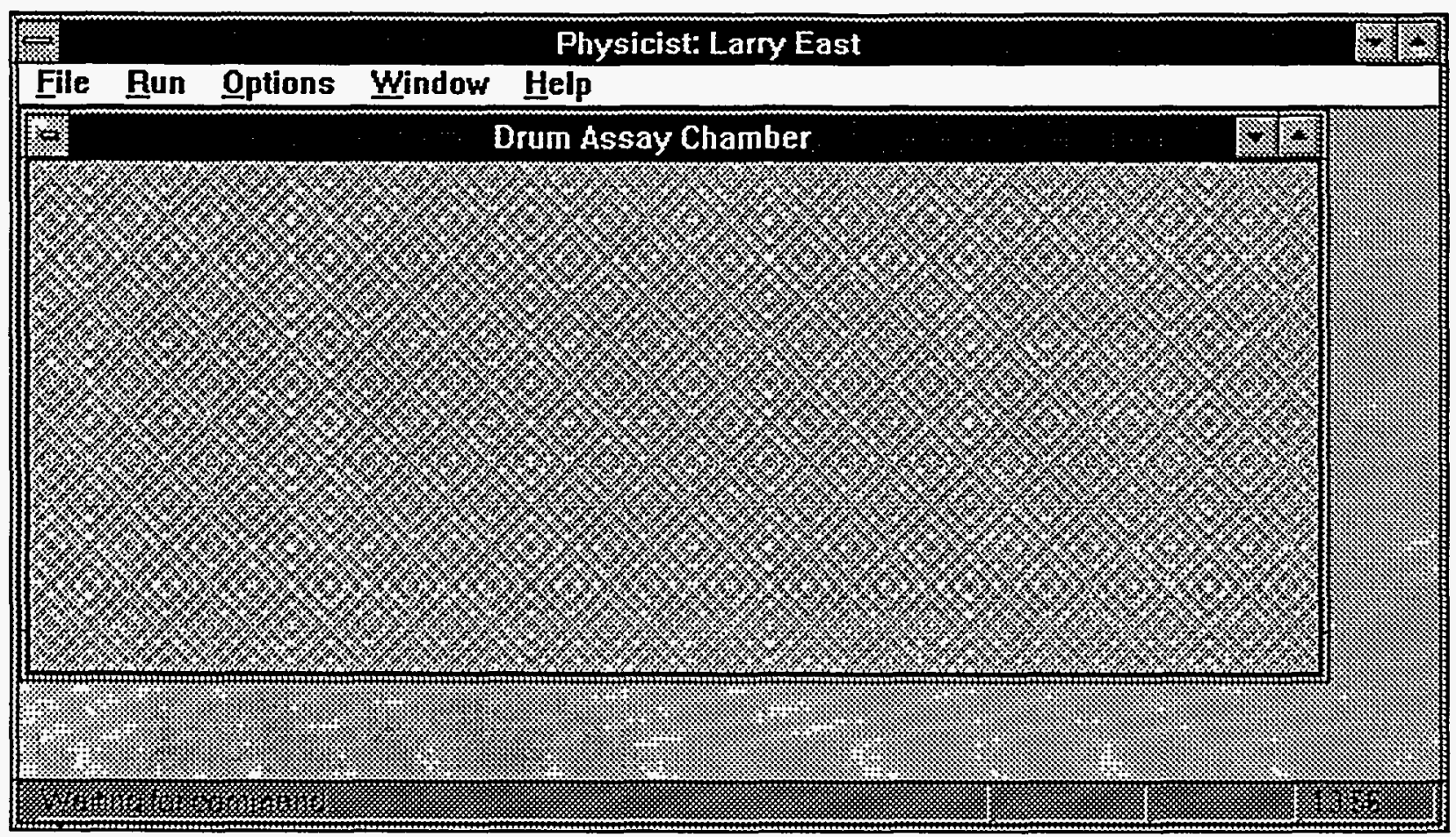

Figure 20. Main window with one chamber as a child window.

\subsection{8 matrix}

\subsubsection{Description}

The matrix class models the waste content of a container. (See Figure 4.) Active and passive signals are used to estimate the amount of plutonium, americium and (in some cases) uranium in the waste. Other derived attributes are computed based on the masses of plutonium, americium and uranium. These include thermal power, thermal power density, total alpha activity, and activity concentration.

The matrix object performs an assay by computing estimates for the plutonium mass based on the active and the passive data. A mass selection criteria determines which of these is reported as the plutonium mass.

\subsubsection{Decomposition}

There is no superclass. There are no derived classes.

\subsubsection{Dependencies}

A matrix object is created and managed by a chamber window. In addition, the matrix list and matrix dialog maintain the list of available matrixes.

The instances used in data reduction must be associated with a chamber in order to access the chamber calibration parameters. The matrix must read the correlation coefficients (for the chamber) from a 
data file, the nuclide properties from a data file, and the mass fraction distribution of plutonium from a data file. Through the association with the chamber, the matrix has access to the active signals and the passive signals. Those signals are required to perform the data reduction.

The matrix is associated with a container that provides the volume used to compute the thermal power density.

The matrix generates a report of the assay results when requested.

\subsubsection{Interfaces}

Files: CORREL.DAC, NUCLIDE.DAT, gamma data file (default DEFAULT.GAM).

Windows: none.

Reports: summary report.

\subsection{9 matrix_dlg}

\subsubsection{Description}

The matrix_dlg object provides the user interface to modify the matrix list. (See Figure 4.) The key attributes of a matrix are the content code, type description, and whether it is a sludge (normal or americium bearing) or non-sludge.

\subsubsection{Decomposition}

This class is derived from CDialog, a class in the MFC library.

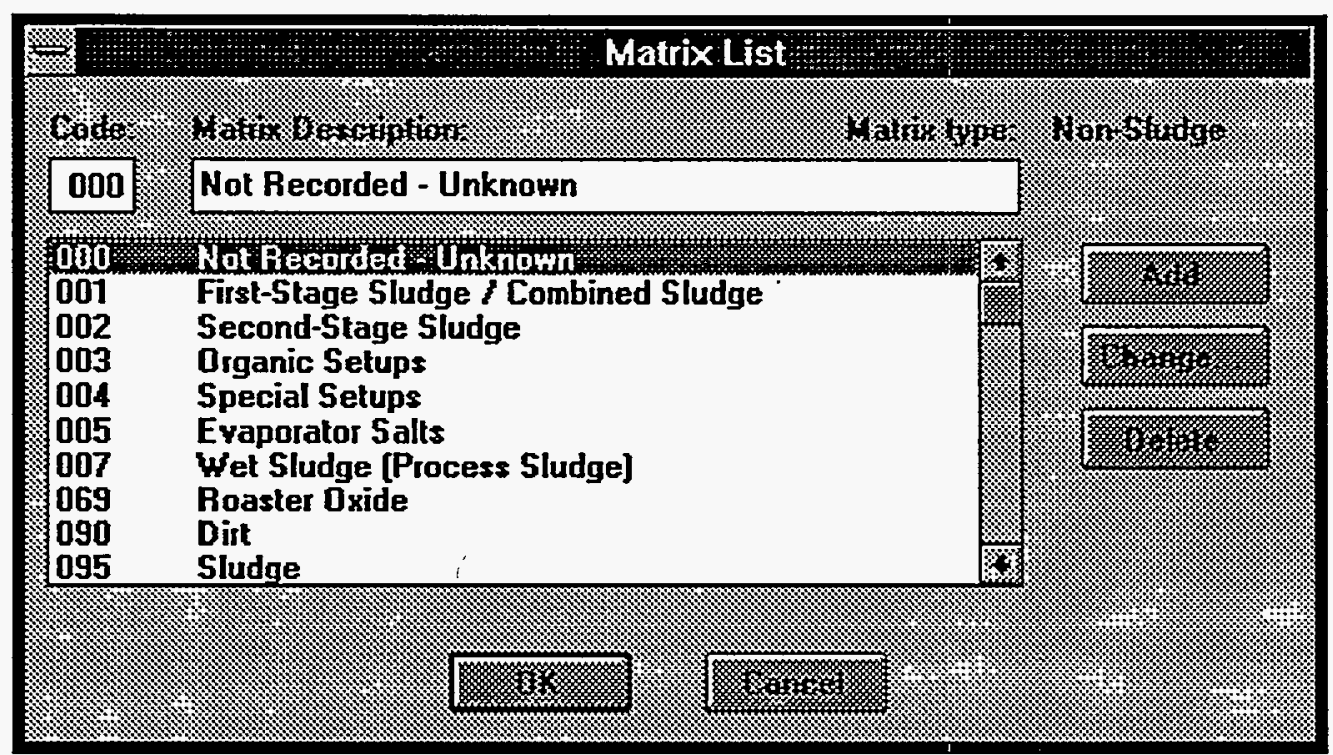

Figure 21. Matrix dialog window. 


\subsubsection{Dependencies}

A matrix_dlg object is created by the main window when the menu item Matrix List... is selected form the "Physicist" menu. The matrix list provides the interface to add, remove, and find matrixes in the list.

\subsubsection{Interfaces}

Files: none.

Windows: the matrix dialog (Figure 21).

\subsection{0 matrix_list}

\subsubsection{Description}

4.)

The matrix list class models the list of matrixes that are processed at the assay station. (See Figure

\subsubsection{Decomposition}

This class is derived from the list class. There are no subclasses.

\subsubsection{Dependencies}

A single instance of the matrix list is created by the main window. This matrix_list is used in the matrix dialog (Figure 20) to allow a Physicist to modify the list and in the waste dialog (Figure 27) to allow the user to select a particular matrix for processing.

\subsubsection{Interfaces}

Files: none.

Windows: none.

\subsection{1 matrix_parameter_dlg}

\subsubsection{Description}

The matrix_parameter_dlg class provides the user interface to modify selected parameters for the matrix class. (See Figure 4.) These parameters are the moderator index, the coefficients for the self absorption correction factor correlation, and the matrix correction factors (active and passive shielded coincidence and passive system coincidence) that are externally computed using a Monte Carlo simulation.

\subsubsection{Decomposition}

This class is derived from CDialog, a class in the MFC library. 


\subsubsection{Dependencies}

This class is created by the matrix dialog whenever these attributes need to be changed.

\subsubsection{Interfaces}

Files: none.

Windows: the matrix parameters dialog (Figure 22).

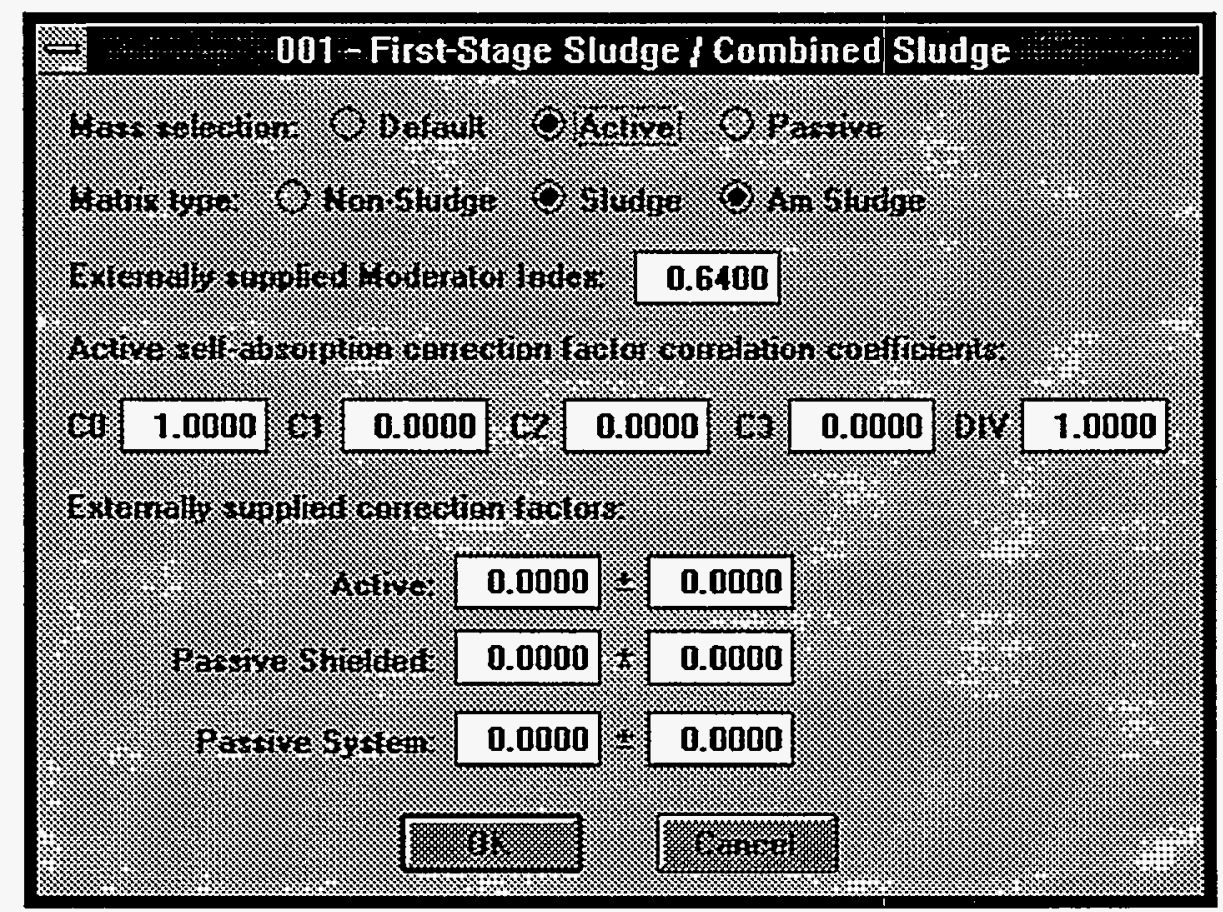

Figure 22. Matrix parameter dialog window.

\subsection{2 message_dlg}

\subsubsection{Description}

The message_dlg object is used to display a message associated with the detection of bad data. This class was added in Version 2.0. The standard MFC dialog box is not used in this case because it was desired to provide a custom icon and custom push buttons in the dialog window.

\subsubsection{Decomposition}

This class is derived from CDialog, a class in the MFC library.

\subsubsection{Dependencies}

A message_dlg object is created by a chamber in response to bad data detected by active_dlg or passive_dlg. 


\subsubsection{Interfaces}

Files: none.

Windows: Message dialog window (Figure 23).

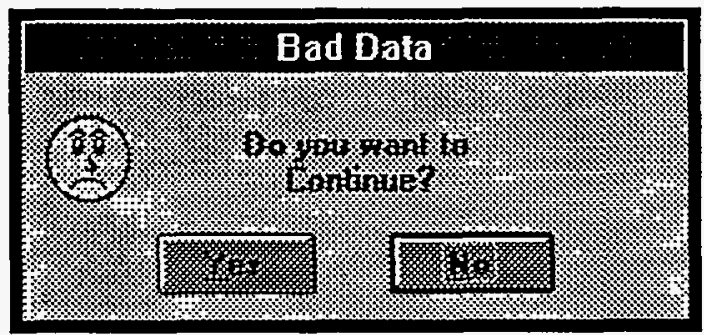

Figure 23. Message dialog window.

\subsection{3 nuclide}

\subsubsection{Description}

The nuclide object encapsulates the attributes and operations for nuclides. (See Figure 4.) The mass of a nuclide is defined as an error bar. Nuclide properties are its mass, mass fraction (for plutonium isotopes), mass ratio (relative to $\mathrm{Pu}-239$ ), auxiliary mass ratio (nuclide dependent), alpha branching ratio, equivalent pu factor, fissile gram equivalent, specific activity and thermal power (several of these attributes were added in Version 2.0).

\subsubsection{Decomposition}

There is no superclass. There are no subclasses.

\subsubsection{Dependencies}

The matrix class creates instances of nuclide that describes the plutonium and americium isotopes.

\subsubsection{Interfaces}

Files: none.

Windows: none.

\subsection{4 options_dlg}

\subsubsection{Description}

The options_dlg class provides the user interface to change the options for data acquisition operations. (See Figure 10.) The options include the background count time, the active pulse count, and the passive count time. 
INEL-96/0057

\subsubsection{Decomposition}

This class is derived from CDialog, a class in the MFC library. There are no subclasses.

\subsubsection{Dependencies}

This class is created by the chamber window in response to selecting the menu item Assay Times....

\subsubsection{Interfaces}

Files: none.

Windows: the processing options dialog (Figure 24).

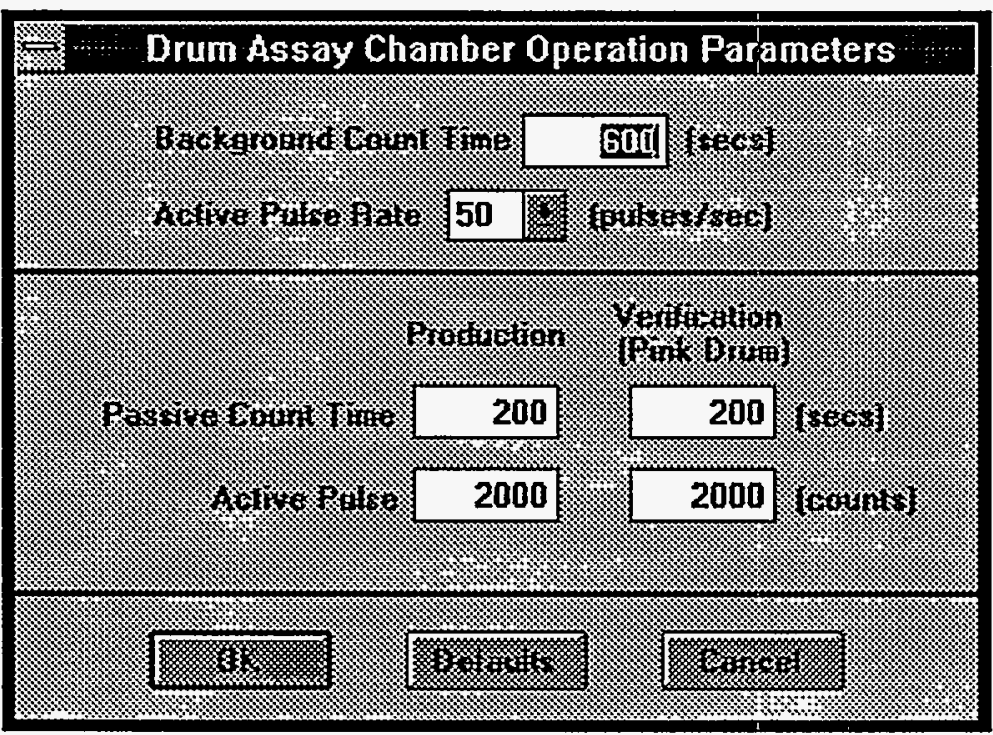

Figure 24. Processing options (assay times) dialog window.

\subsection{5 passive_dlg}

\subsubsection{Description}

The passive dialog shows the counts and rates from a passive data acquisition. (See Figure 10.) Both the bare and shielded counts are displayed for each of the six sides and the total. Rates for the shielded total, the system total, the shielded coincidence, and system coincidence are displayed with an error of one standard deviation. The sums of the six sides are checked against the CAMAC hardware totals. Additionally, the variation of the four vertical sides is checked against the average for both bare and shielded detectors.

\subsubsection{Decomposition}

This class is derived from CDialog, a class in MFC library. There are no subclasses. 


\subsubsection{Dependencies}

This object is created by a chamber window when passive data are acquired or read from a file. The passive dialog creator function receives a pointer to a passive signals object through the argument list. The passive signals pointer provides the dialog with the interface to get the counts and rates that the dialog displays. The passive dialog window is a child window of the creating chamber window.

\subsubsection{Interfaces}

Files - none.

Windows - the passive dialog window (Figure 25) and, optionally, diagnostic message boxes.

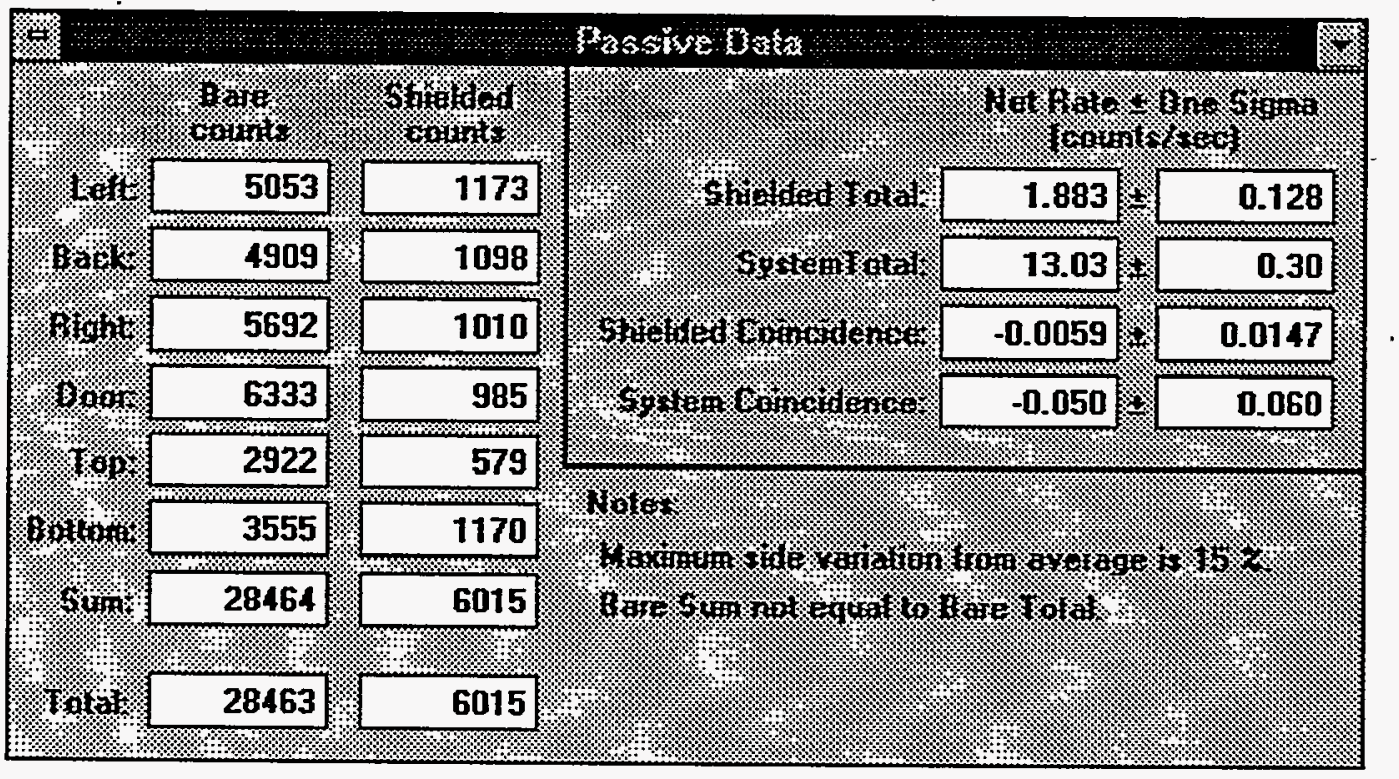

Figure 25. Passive dialog (data) window.

\subsection{6 passive_signals}

\subsubsection{Description}

The passive_signals class computes the net rates for the shielded total, the system total, the shielded coincidence, and the system coincidence signals. (See Figure 5.)

\subsubsection{Decomposition}

This class is derived from the signals class. There are no subclasses.

\subsubsection{Dependencies}

A passive_signals object is created by a chamber object. Pointers to the associated camac_crate object that maintains the passive gross data and the passive background data are passed as arguments to 


\section{INEL-96/0057}

the passive_signals creator function. The camac_crate provides the interface to get the gross counts, background counts, and count rates for the passive signals.

The shielded coincidence count is associated with the "short gate," and the system coincidence count is associated with the "long gate." These gates and the signal names are defined in the CAMAC crate configuration file. A change in the naming convention of the passive signals will require a change in the passive signals class.

The passive signals object is used by the passive_dlg object to display the passive data, and it is used by the matrix object in the data reduction.

\subsubsection{Interfaces}

Files - none.

Windows - none.

\subsection{7 port}

\subsubsection{Description}

The port class models a generic port of a CAMAC module. (See Figure 9.) Each port has a name.

\subsubsection{Decomposition}

There is no superclass. Subclasses are clock_port, gate_port, and scaler_port.

\subsubsection{Dependencies}

CAMAC objects create ports appropriate to the type of the module being modeled. A camac_crate can search the ports via the interface provided by each module type.

\subsubsection{Interfaces}

Files: none.

Windows: none.

\subsection{8 port_2323}

\subsubsection{Description}

The port_2323 class models the gate port associated with a camac_2323. (See Figure 9.)

\subsubsection{Decomposition}

This class is derived from the gate_port class. There are no subclasses.

\subsubsection{Dependencies}

A camac_2323 object creates an array of port_2323 objects. 


\subsubsection{Interfaces}

Files: none.

Windows: none.

\subsection{9 results_dig}

\subsubsection{Description}

The results_dlg provides the user interface to view the results of a data analysis. (See Figure 10.) It displays the total plutonium and isotopic masses, uranium isotopic mass (if present) americium-241 mass, thermal power, thermal power density, activity values and fissile gram equivalent value. Each quantity is displayed with an error of one standard deviation.

The dialog provides push buttons to print the summary report, save the data file, and close the file. The summary report is automatically printed if the chamber window menu item summary report is checked. Data are automatically saved to a file at the end of an assay if the automatic save item is check in the chamber window menu The save and close functions are also provided under the chamber window's File menu.

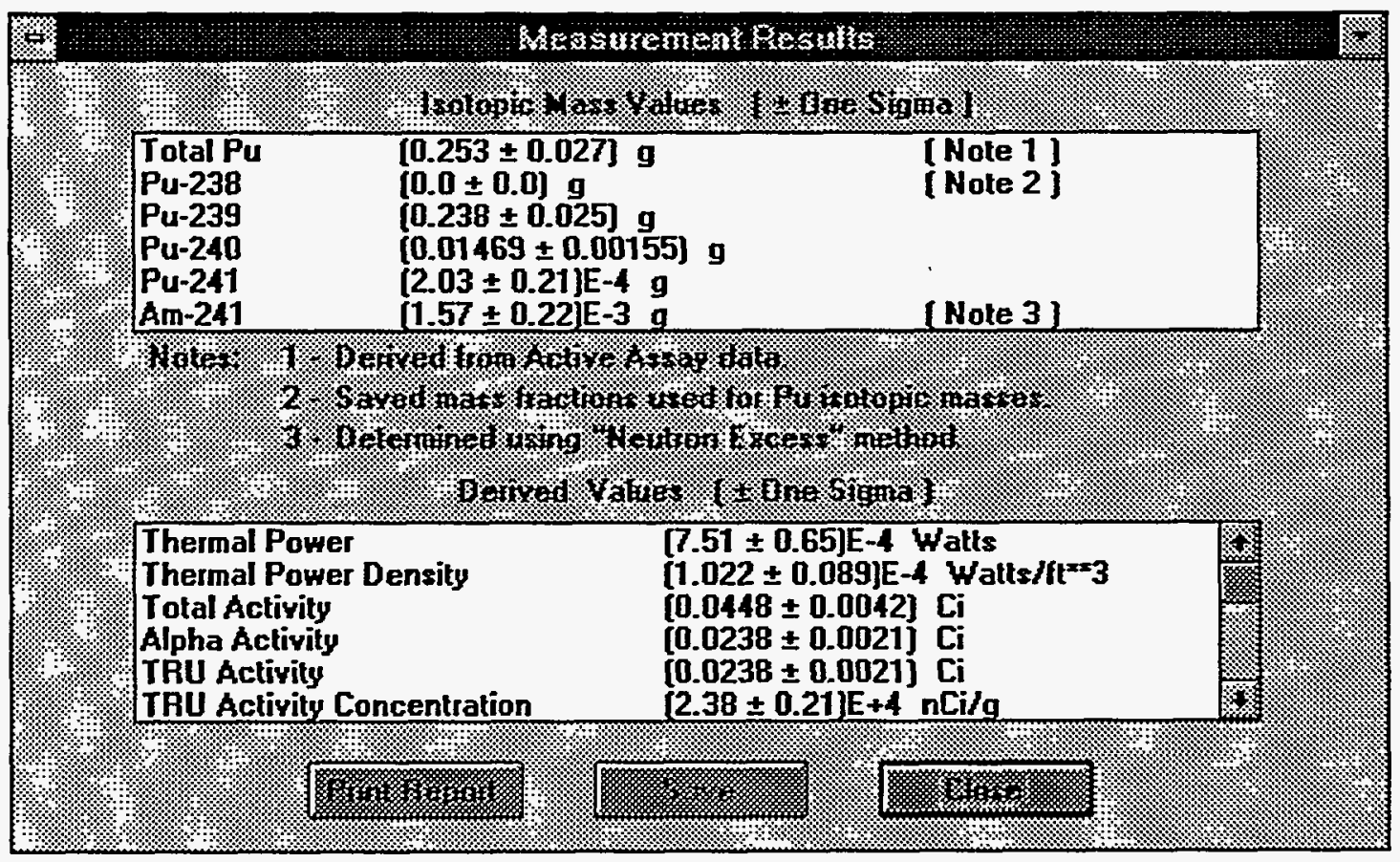

Figure 26. Results dialog window.

\subsubsection{Decomposition}

This class is derived from CDialog, a class in the MFC library. There are no subclasses. 
INEL-96/0057

\subsubsection{Dependencies}

A results_dlg object is created at the completion of a data reduction by the chamber window. This dialog is declared as a friend class in matrix so that it can access the quantities to be displayed.

\subsection{9 .4 Interfaces}

Files: none.

Windows: the results dialog (Figure 26).

\subsection{0 scaler}

\subsubsection{Description}

The scaler class models a generic CAMAC scaler module. (See Figure 8.) It defines the interface to clear and read the scaler registers.

\subsubsection{Decomposition}

This class is derived from the camac class. Subclasses are camac_2551 and camac_450.

\subsubsection{Dependencies}

A scaler is associated with an array of scaler ports. This provides the interface to get and set the counts for individual ports.

\subsubsection{Interfaces}

Files: none.

Windows: none.

\subsection{1 scaler_port}

\subsubsection{Description}

The scaler_port class models the behavior of a port in a CAMAC scaler module. (See Figure 9.) The scaler port manages a device list.

\subsubsection{Decomposition}

This class is derived from the port class. There are no subclasses.

\subsubsection{Dependencies}

A scaler has an array of scaler ports. Each scaler port is associated with either a clock port or a gate port. The scaler_port manages a device list that provides the interface from which the camac_crate object can identify specific devices and retrieve counts, rates, and times. 
The scaler_port provides the interface to find specific devices.

\subsubsection{Interfaces}

Files: none.

Windows: none.

\subsection{2 signals}

\subsubsection{Description}

The signals class models the generic functionality of the signals needed for data reduction. (See Figure 5.)

\subsubsection{Decomposition}

There is no superclass. Subclasses are active_signals and passive_signals.

\subsubsection{Dependencies}

There are no dependencies. The signals class is not intended to be instantiated. Rather, it abstracts certain behavior common to both the active and passive signals.

\subsubsection{Interfaces}

Files: none.

Windows: none.

\subsection{3 timer}

\subsubsection{Description}

The timer class defines the behavior of a (CAMAC) logical timer. (See Figure 7.)

\subsubsection{Decomposition}

This class is derived from the device class. There are no subclasses.

\subsubsection{Dependencies}

A timer object is associated with a scaler port by virtue of its inherited properties from device and with a clock port. The timer computes a time using the counts from the scaler port and the clock rate from the clock port.

\subsubsection{Interfaces}

Files: none.

Windows: none. 


\subsection{4 user}

\subsubsection{Description and Purpose}

The user class defines the user name, ID, password, and access level for each user of the assay system. (See Figure 2.) The class reads and writes the user attributes in a data file.

\subsubsection{Decomposition}

There is no superclass. There are no derived classes.

\subsubsection{Dependencies}

The user object created by the main window is the login user, whose access level determines the functions that may be performed. The user list creates instances of user to define the list of all registered users. The users dialog creates instances of user to add to the user list.

\subsubsection{Interfaces}

Files: none.

Windows: none.

\subsection{5 users_dlg}

\subsubsection{Description and Purpose}

The users_dialog class provides the interface for the administrator to modify the list of users and user attributes. (See Figure 2.)

\subsubsection{Decomposition}

This class is derived from CDialog, a class in the MFC library. There are no subclasses.

\subsubsection{Dependencies}

The users dialog is created in the main window when a user with Administrator access level selects the menu item User List .... The dialog access users from the user list

\subsubsection{Interfaces}

Files: none.

Windows: the users dialog.

\subsection{6 user_list}

\subsubsection{Description}

The user_list class maintains the list of users who have access to the assay system. (See Figure 2.) This is not a "secure" list; primary system access control is accomplished by facility access procedures. 


\subsubsection{Decomposition}

This class is derived from the list class. There are no subclasses.

\subsubsection{Dependencies}

The main window manages the global user list. The main window login function validates a login request against the user list.

Through the users dialog, the user list may be modified and written to its file, or it may be reread.

\subsubsection{Interfaces}

Files: USER.LST.

Windows: none.

\subsection{7 waste_dlg}

\subsubsection{Description}

The waste dlg class provides the user interface to identify the waste container type, matrix type, and net weight of the container being processed. (See Figure 10.)

For new files, the user may input or select the attributes. The user selects the container type in a list selection box. The volume of a container is displayed, but cannot be modified through the waste dialog. The user selects the matrix type in another list selection box.

For an existing file, the users with Operator access privilege may not change the attributes, but they can be changed by a Physicist after selecting the "Edit" button at the bottom of the window (not shown to Operators)..

\subsubsection{Decomposition}

This class is derived from CDialog, a class in the MFC library.

\subsubsection{Dependencies}

The chamber window creates a waste dialog to process a new waste container or to perform recalculation on a previously processed container. The waste dialog calls the methods of both the container and the matrix classes to define a waste container for processing. The waste dialog also uses the global pointers to the container list and the matrix list.

\subsubsection{Interfaces}

Files: none.

Windows: the waste dialog (Figure 27). 


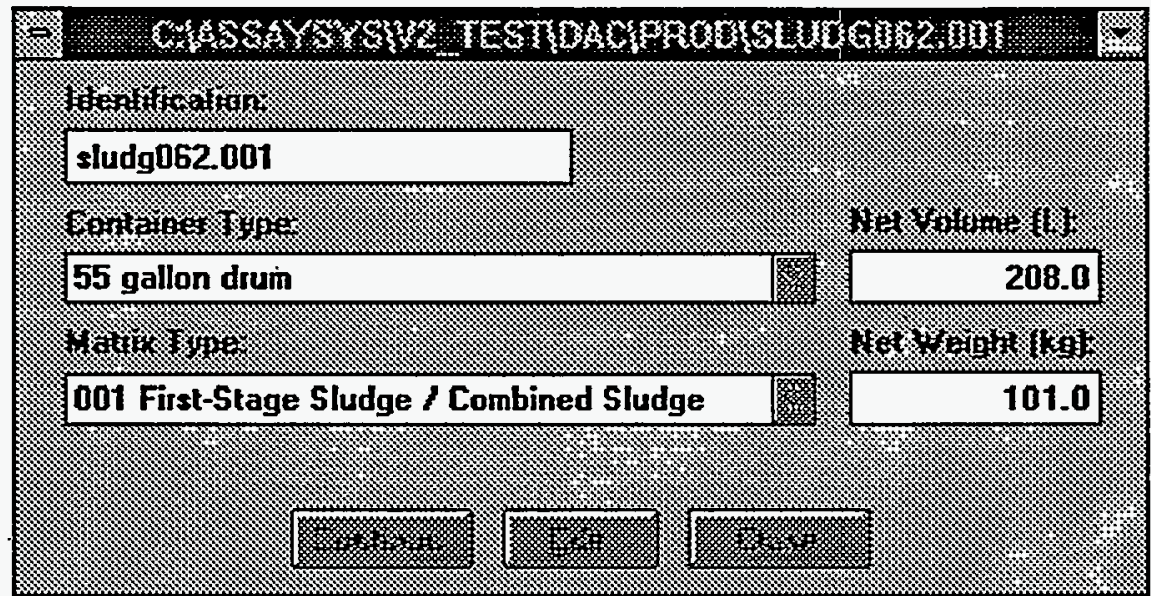

Figure 27. Waste dialog window. 


\section{REQUIREMENTS TRACEABILITY}

The requirements traceability matrix in Table 3 identifies the requirement name and paragraph number from the software requirements specification (SRS), Reference [3]. The third column identifies the object where the requirement is designed and coded.

Table 3. Requirements traceability matrix.

\begin{tabular}{|c|c|c|}
\hline SRS & Requirement & SDD / code \\
\hline 3.1.2.2 M.1 & Login users & $\begin{array}{l}\text { login_dlg, user, user_list, } \\
\text { main_wnd }\end{array}$ \\
\hline 3.1.2.2 M.2 & Present user menu functions & main_wnd \\
\hline 3.1.2.2 M.3 & Logout users & main_wnd \\
\hline 3.1.2.2 M.4 & Allow Administrator to modify user list & $\begin{array}{l}\text { users_dlg, user, user_list, } \\
\text { main_wnd }\end{array}$ \\
\hline 3.1.3.2 M.1 & Read default processing options for assay system & main_wnd, chamber_wnd \\
\hline 3.1.3.3 M.1 & Read default chamber processing options & chamber_wnd, options_dlg \\
\hline 3.1.3.3 M.2 & $\begin{array}{l}\text { Allow an Operator or Physicist to modify the } \\
\text { processing options }\end{array}$ & chamber_wnd, options_dlg \\
\hline 3.1.3.3 M.3 & Allow Physicist to modify the chamber calibration & $\begin{array}{l}\text { calibration_dlg, } \\
\text { chamber_wnd }\end{array}$ \\
\hline 3.1.3.3 M.4 & Perform chi-square test & chamber_wnd \\
\hline 3.1.3.4 M.1 & Read CAMAC crate configuration from file & camac_crate \\
\hline 3.1.3.4 M.2 & Get time and date of data acquisition & camac_crate \\
\hline 3.1.3.4 M.3 & Acquire active data from assay chamber & chamber, camac_crate \\
\hline 3.1.3.4 M.4 & Store active data on file & camac_crate \\
\hline 3.1.3.4 M.5 & Read active data from file & camac_crate \\
\hline 3.1.3.4 M.6 & Acquire passive data from assay chamber & chamber, camac_crate \\
\hline 3.1.3.4 M.7 & Store passive data on file & chamber, camac_crate \\
\hline 3.1.3.4 M.8 & Read passive data from file & camac_crate \\
\hline 3.1.3.5 M.1 & $\begin{array}{l}\text { Compute flux monitor gross, background, and net } \\
\text { rates }\end{array}$ & active_signals, detector \\
\hline 3.1.3.5 M.2 & $\begin{array}{l}\text { Compute flux monitor gross, background, and net } \\
\text { rate errors }\end{array}$ & $\begin{array}{l}\text { active_signals, detector, } \\
\text { error_bar }\end{array}$ \\
\hline 3.1.3.5 M.3 & $\begin{array}{l}\text { Compute barrel flux monitor gross, background, } \\
\text { and net rates }\end{array}$ & active_signals, detector \\
\hline 3.1.3.5 M.4 & $\begin{array}{l}\text { Compute barrel flux monitor gross, background, } \\
\text { and net rate errors }\end{array}$ & $\begin{array}{l}\text { active_signals, detector, } \\
\text { error_bar }\end{array}$ \\
\hline 3.1.3.5 M.5 & $\begin{array}{l}\text { Compute shielded total gross, background, and net } \\
\text { rates }\end{array}$ & active_signals, detector \\
\hline
\end{tabular}




\begin{tabular}{|c|c|c|}
\hline SRS & Requirement & SDD / code \\
\hline 3.1.3.5 M.6 & $\begin{array}{l}\text { Compute shielded total gross, background, and net } \\
\text { rate errors }\end{array}$ & $\begin{array}{l}\text { active_signals, detector, } \\
\text { error_bar }\end{array}$ \\
\hline 3.1.3.6 M.1 & Compute shielded total rate & passive_signals, detector \\
\hline 3.1.3.6 M.2 & Compute shielded total rate error & $\begin{array}{l}\text { passive_signals, } \\
\text { detector,error_bar }\end{array}$ \\
\hline 3.1.3.6 M.3 & Compute system total rate & passive_signals, detector \\
\hline 3.1.3.6 M.4 & $\begin{array}{l}\text { Compute gross, background, and net system total } \\
\text { rate error }\end{array}$ & $\begin{array}{l}\text { passive_signals, detector, } \\
\text { error_bar }\end{array}$ \\
\hline 3.1.3.6 M.5 & Compute shielded coincidence rate & passive_signals, detector \\
\hline 3.1.3.6 M.6 & Compute shielded coincidence rate error & $\begin{array}{l}\text { passive_signals, detector, } \\
\text { error_bar }\end{array}$ \\
\hline 3.1.3.6 M.7 & Compute system coincidence rate & passive_signals, detector \\
\hline 3.1.3.6 M.8 & Compute system coincidence rate error & $\begin{array}{l}\text { passive_signals, detector, } \\
\text { error_bar }\end{array}$ \\
\hline 3.1.4.2 M.1 & Input container attributes & $\begin{array}{l}\text { waste_dlg, container, } \\
\text { chamber_wnd }\end{array}$ \\
\hline 3.1.4.2 M.2 & Store container data on file & container \\
\hline 3.1.4.2 M.3 & Read container data from file & container \\
\hline 3.1.4.2 M.4 & Provide container summary report & container \\
\hline 3.1.4.2 M.5 & Allow Physicist to modify the container list & $\begin{array}{l}\text { container_dlg, container, } \\
\text { container_list, main_wnd }\end{array}$ \\
\hline 3.1.4.3 M.1 & Input matrix attributes & $\begin{array}{l}\text { waste_dlg, matrix, } \\
\text { chamber_wnd }\end{array}$ \\
\hline 3.1.4.3 M.2 & Store matrix data on file & matrix \\
\hline 3.1.4.3 M.3 & Read matrix data from file & matrix \\
\hline 3.1.4.3 M.4 & Allow Physicist to modify the matrix list & $\begin{array}{l}\text { matrix_dlg, matrix, } \\
\text { matrix_list, main_wnd }\end{array}$ \\
\hline 3.1.4.3 M.5 & Calculate active $\mathrm{Pu}$ mass & matrix \\
\hline 3.1.4.3 M.6 & Calculate active $\mathrm{Pu}$ mass error & matrix, error_bar \\
\hline 3.1.4.3 M.7 & Calculate passive $\mathrm{Pu}$ mass & matrix \\
\hline 3.1.4.3 M.8 & Calculate passive $\mathrm{Pu}$ mass error & matrix, error_bar \\
\hline 3.1.4.3 M.9 & Select either the active or passive Pu mass & matrix \\
\hline 3.1.4.3 M.10 & Select either the active or passive $\mathrm{Pu}$ mass error & matrix, error_bar \\
\hline 3.1.4.3 M.11 & Calculate plutonium isotopic masses & matrix \\
\hline 3.1.4.3 M.12 & Calculate plutonium isotopic mass errors & matrix, error_bar \\
\hline 3.1.4.3 M.13 & Calculate U-235 mass & matrix \\
\hline 3.1.4.3 M.14 & Calculate U-235 mass error & matrix, error_bar \\
\hline 3.1.4.3 M.15 & Calculate the Am-241 mass & matrix \\
\hline 3.1.4.3 M.16 & Calculate the Am-24l mass error & matrix, error_bar \\
\hline 3.1.4.3 M.17 & Calculate thermal power & matrix \\
\hline
\end{tabular}




\begin{tabular}{lll}
\hline \multicolumn{1}{c}{ SRS } & \multicolumn{1}{c}{ Requirement } & \multicolumn{1}{c}{ SDD / code } \\
\hline 3.1.4.3 M.18 & Calculate the thermal power error & matrix, error_bar \\
3.1.4.3 M.19 & Calculate the thermal power density & matrix \\
3.1.4.3 M.20 & Calculate the thermal power density error & matrix, error_bar \\
3.1.4.3 M.21 & Calculate activity values & matrix \\
3.1.4.3 M.22 & Calculate the activity errors & matrix, error_bar \\
3.1.4.3 M.23 & Calculate the TRU activity concentration & matrix \\
3.1.4.3 M.24 & Calculate the TRU activity concentration error & matrix, error_bar \\
3.1.4.3 M.25 & Calculate Fissile Gram Equivalent & matrix \\
3.1.4.3 M.26 & Calculate Fissile Gram Equivalent error & matrix, error_bar \\
3.1.4.3 M.27 & Provide a measurement summary report for the & matrix \\
& container & dms_dlg \\
3.1.5.2 M.1 & Send "handshake" message to DMS & dms_dlg, chamber_wnd \\
3.1.5.2 M.2 & Send measurement results to DMS & dms_dlg \\
3.1.5.2 M.3 & Receive "handshake" message from DMS & dms_dlg \\
3.1.5.2 M.4 & Receive new assay request from DMS & dms_dlg \\
3.1.5.2 M.5 & Receive recalculation request from DMS & \\
\hline
\end{tabular}




\section{DESIGN DETAIL}

An overview of the software design can be seen by examining the header or "include" files that define $\mathrm{C}+$ objects that make up the SAS software. A header file is associated with each class object developed for the program. Each header file identifies the public and private attributes and methods for each class. Associations with other classes are defined, typically through a $\mathrm{C}$ language pointer. $\mathrm{A}$ header file also identifies any superclass from which the class is derived, often a class defined in the Microsoft (B) Foundation Class (MFC) Library that is part of the Visual C+$+^{\mathrm{TM}}$ development platform [16]. Appendix A lists each header file used in the SAS software.

\section{FUTURE DEVELOPMENT DIRECTIONS}

It is expected that this software will continue to evolve to support hardware changes planned for the SWEPP assay and DMS systems.

During the development of Version 2.0 of the SAS software, it became evident that some structural changes should be considered in order to reduce the complexity of the program objects. A "metrics study" performed on the source code about half way through the Version 2.0 upgrade development process indicated that the program was becoming less maintainable do to increasing complexity [15]. No restructuring was attempted due to time and resource constraints. Two obvious targets for complexity reduction are the "matrix" and "chamber_wnd" objects. The chamber_wnd object controls, directly or indirectly, most of the data acquisition and many of the user interface functions of the program. The matrix object contains all of the data reduction algorithms in addition to operations dealing directly with matrix properties of the waste container being measured. The feasibility of putting some of the functionality of these class objects into new (possibly derived) classes should be investigated. Program complexity could also be reduced by removing the option to emulate NEUT2 program behavior.

Functions that send output to a printer should be changed to use standard Windows ${ }^{\top \mathrm{M}}$ printer support functions in order to take advantage of the high degree of device independence offered by these functions. At the present time, all printing is encapsulated in the "hstreambuf" object which was written specifically for HP LaserJet III and compatible printers.

Moving from the Windows platform to Windows-NT ${ }^{\top M}$ should be considered in order to take advantage of the security features and enhanced network and serial communications support available in Windows-NT. 


\section{Appendix A}

\section{Header Files}

A-1 


\section{Appendix A}

\section{Header Files}

Listings of the following program header files are contained in this appendix:

$\begin{array}{lll}\text { ABOUTDLG.H } & \text { ACQRDLG.H } & \text { ACTDLG.H } \\ \text { CALIBDLG.H } & \text { CAM217.H } & \text { CAM2323.H } \\ \text { CAM8901.H } & \text { CAMAC.H } & \text { CAMCRATE.H } \\ \text { CIOCKGEN.H } & \text { CLOKPORT.H } & \text { COINDET.H } \\ \text { CONTLIST.H } & \text { CONTROL.H } & \text { DEIAYDLG.H } \\ \text { DEVLIST.H } & \text { DMSDLG.H } & \text { ERRORBAR.H } \\ \text { GATEGEN.H } & \text { GATEPORT.H } & \text { HSTREAM.H } \\ \text { LIST.H } & \text { LOGINDLG.H } & \text { MAINWND.H } \\ \text { MATRIX.H } & \text { MATRIIST.H } & \text { MENU.H } \\ \text { PASDLG.H } & \text { PASSGNLS.H } & \text { PORT.H } \\ \text { RANGECHK.H } & \text { RESITDLG.H } & \text { RESOURCE.H } \\ \text { SCAIPORT.H } & \text { SIGNALS.H } & \text { TIMER.H } \\ \text { USERSDLG.H } & \text { WASTEDIG.H } & \end{array}$

ACTSGNLS.H

CAM2551.H

CHAMBER. $\mathrm{H}$

CONTAIN.H

DETECTOR. H

FILELIM. $\mathrm{H}$

ITSTREAM. $\mathrm{H}$

MAPARDLG . $\mathrm{H}$

MSGDLG . $\mathrm{H}$

PORT2323. H

RESRC1.H

USER.H
ASSAYSYS . H

CAM450.H

CHAMBWND. $\mathrm{H}$

CONTDIG. $\mathrm{H}$

DEVICE. H

GATE. $\mathrm{H}$

LINK. H

MATRDLG.H

NUCLIDE.H

PROPTDLG.H

SCALER. H

USERLIST.H 


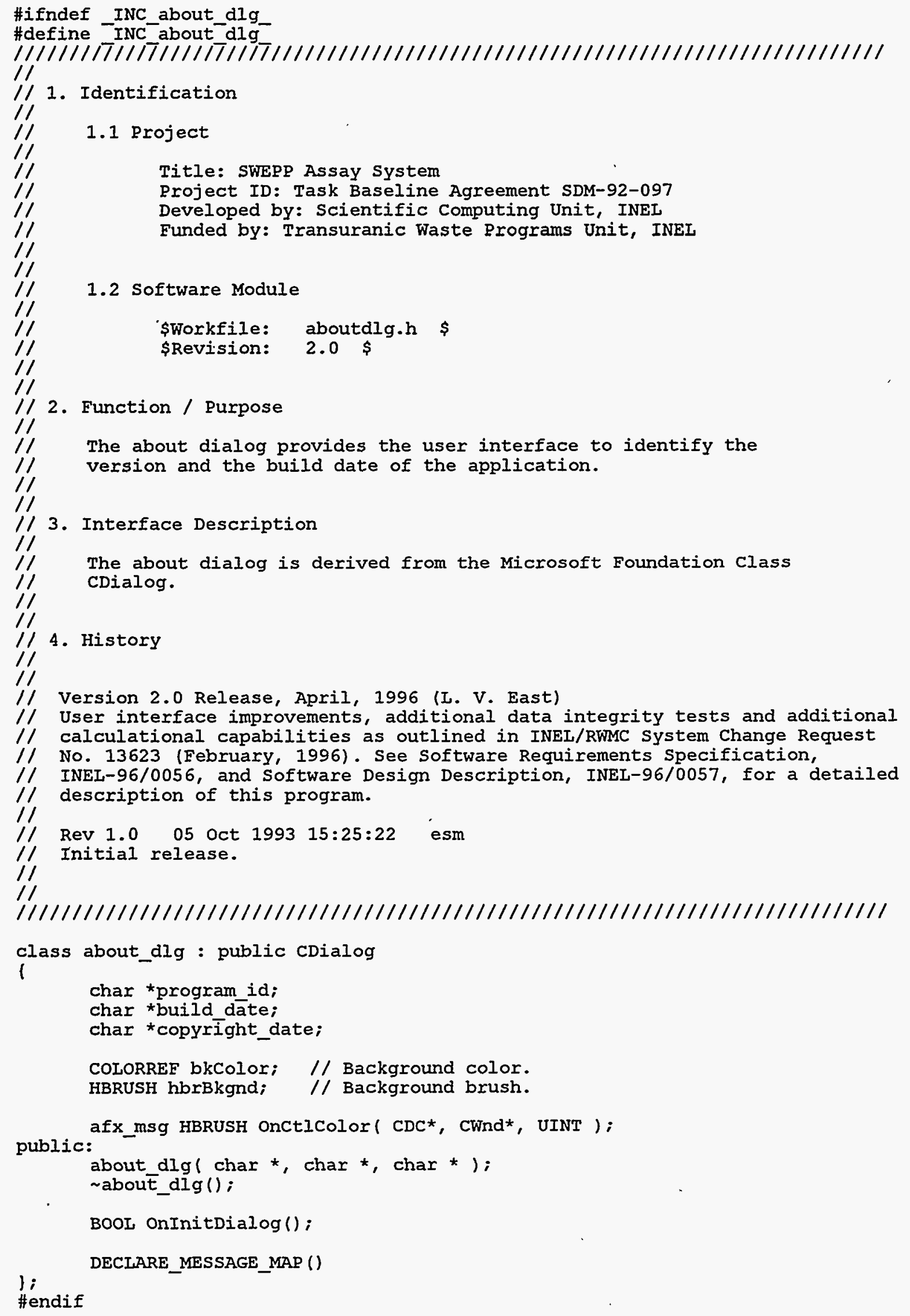


int update_time;

CRect * rectstatus;

CRect * rectBound;

char avalue [8];

BOOL cancel flag;

BOOL in progress:

BOOL do_background;

void draw_background bar ( CDC * );

void draw status bar( CDC *);

void start acquisition();

void Cancelacquisition():

\#if defined( IANL)

HWND $m$ hServer;

CWnd $\star \overline{\text { Getsmc }}()$;

\#endif

void Startserver();

afx msg void onpaint ():

afx msg void onMove ( int, int );

afx_msg void ondestroy();

public:

acquire dlg( chamber *);

acquire_dlg():

void set_title( char *title) \{ this->title = title; \}

void set pulse rate( unsigned long pulse rate)

( this->pulse rat
void check progress();

BOoL OnInitDialog ();

void OnCancel();

\#if defined( IANL)

afx msg LRESULT onserverstarted( WPARAM wparam, IPARAM Iparam ); afx_msg LRESULT onRotationstarted( WPARAM wparam, IPARAM Iparam ); \#endif afx_msg IRESULT OnRotationstopped( WPARAM wparam, IPARAM Iparam );

J

DECLARE_MESSAGE_MAP()

\#endif

\#ifndef INC_active_dlg_

\#define -INC_active_dlg_

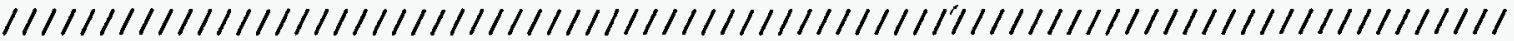

$1 /$

// 1. Identification

$1 /$

// 1.1 Project

$1 /$

$1 / \quad$ Title: SWEPP Assay system

// Project ID: Task Baseline Agreement SDM-92-097

// Developed by: Scientific Computing Unit, INEL

// Funded by: Transuranic Waste Programs Unit, INEI

11

// 1.2 Software Module

$1 /$

I/ \$Workfile: actdlg.h $\$$

// \$Revision: 2.0 \$




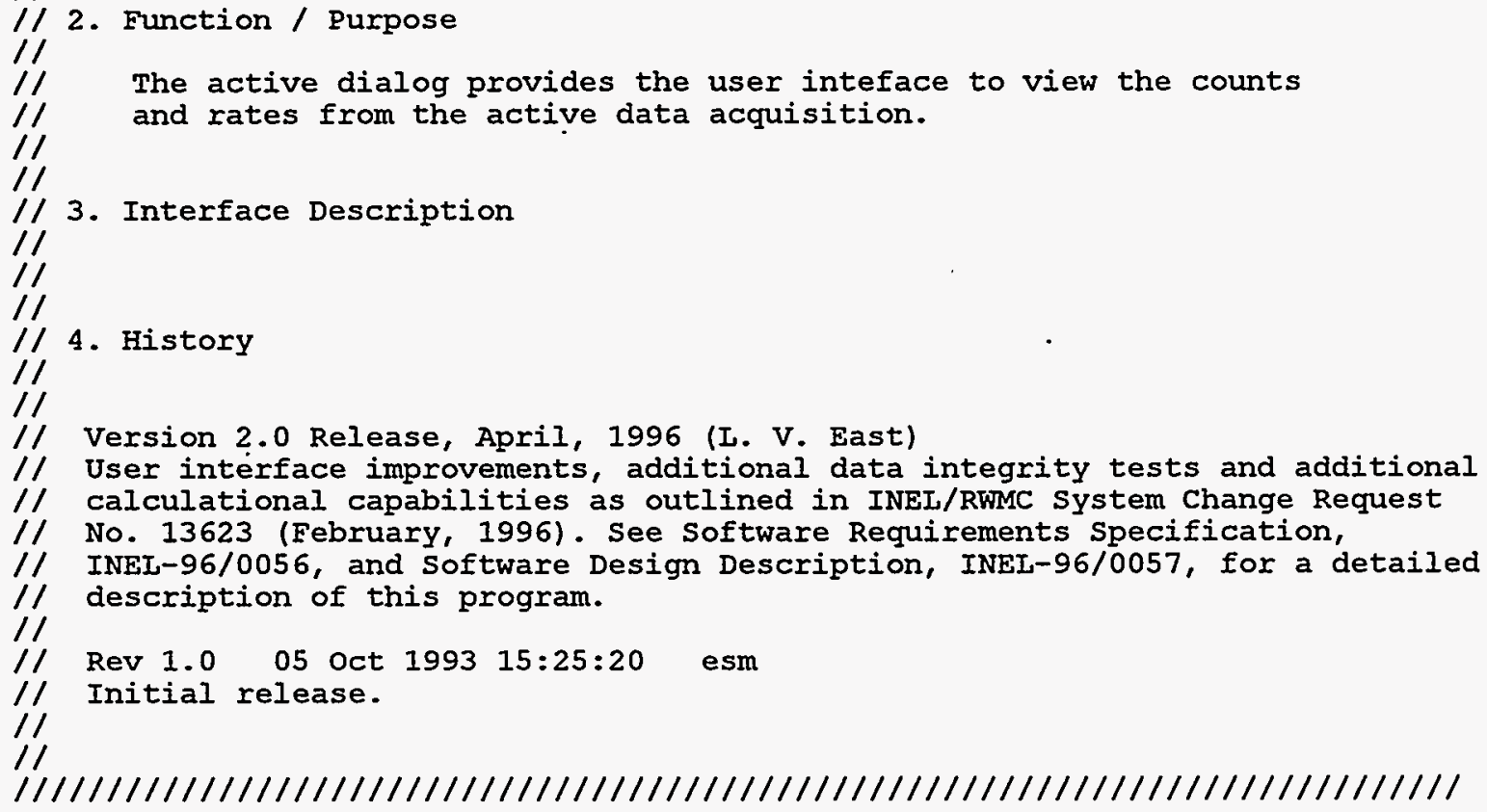


):

DECLARE_MESSAGE_MAP()

\#endif

\#ifndef_INC_active_signals_

\#define IINC_active_signals_

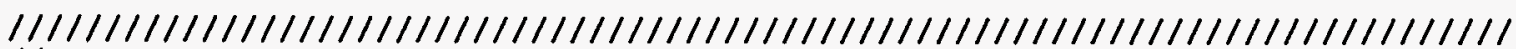

$1 /$

// 1. Identification

11

//

$1 /$

1.1 Project

Title: SWEPP Assay System

Project ID: Task Baseline Agreement SDM-92-097

Developed by: Scientific computing Unit, INEL

Funded by: Transuranic Waste Programs Unit, INEL

1.2 Software Module

\$Workfile: actsgnls.h \$

\$Revision: 2.0 \$

2. Function / Purpose

The active signals provides the interface to the active data to access the shielded total, the flux monitor, and the barrel flux monitor signals.

3. Interface Description

4. History

11

$1 /$

// Version 2.0 Release, April, 1996 (L. V. East)

// User interface improvements, additional data integrity tests and additional

// calculational capabilities as outlined in INEL/RWMC system Change Request

// No. 13623 (February, 1996). See Software Requirements Specification,

// INEL-96/0056, and Software Design Description, INEL-96/0057, for a detailed

// description of this program.

$1 /$

// Rev $1.0 \quad 05$ Oct $1993 \quad 15: 25: 32$ esm

// Initial release.

$1 /$

11

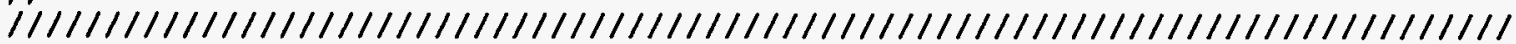

\#pragma hdrstop

\#include "signals.h"

\#include "camcrate. $h$ "

\#include "errorbar.h"

class active_signals : public signals

l

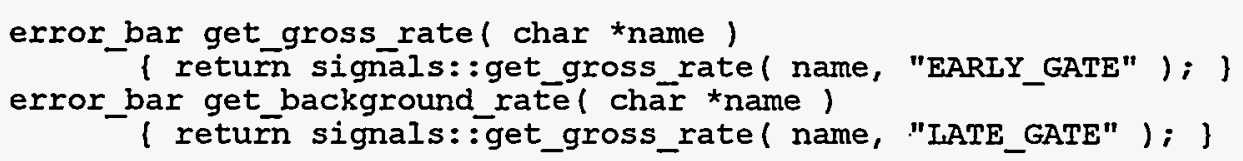




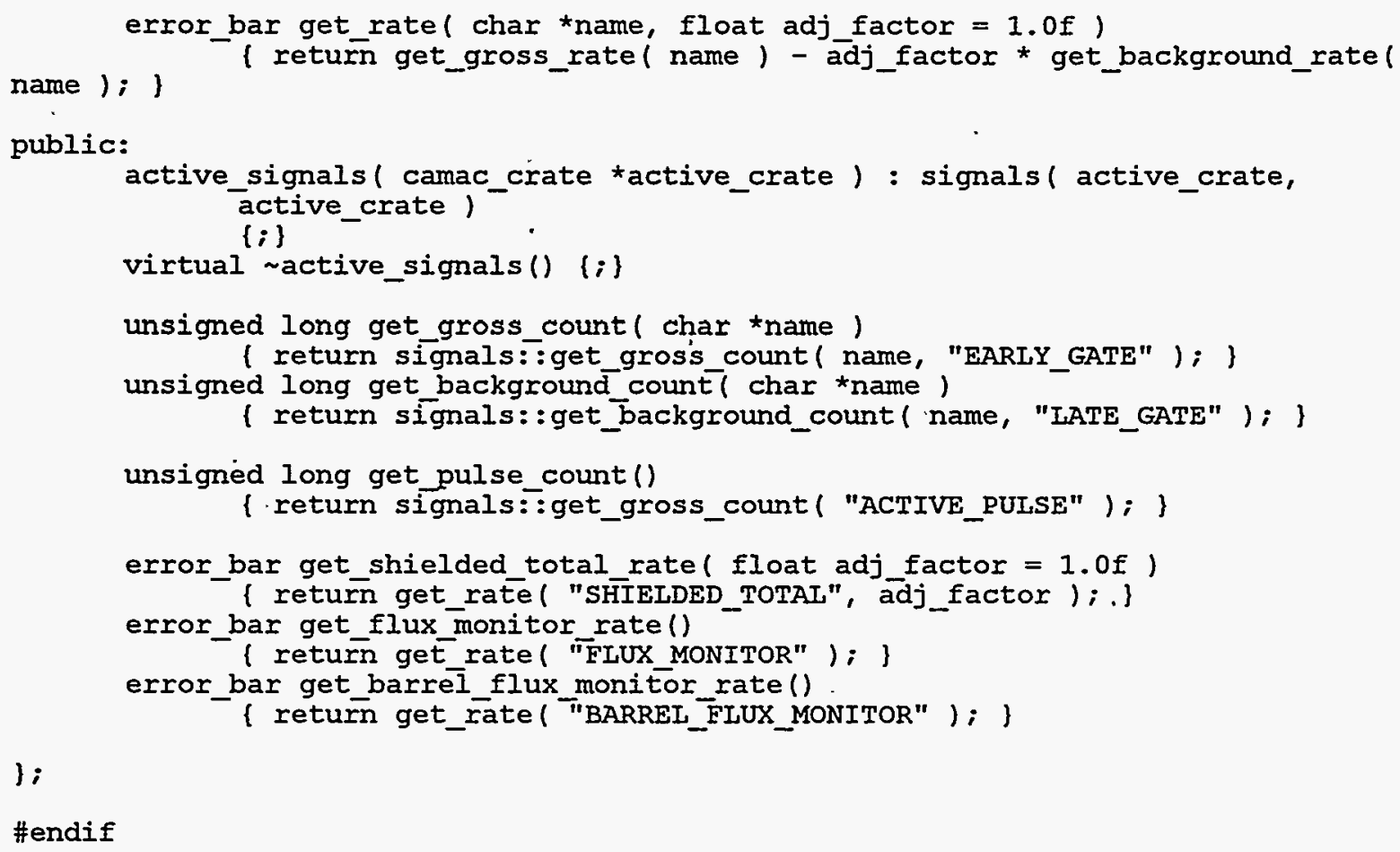

$1 /$

// 2. Function / Purpose

// Version 2.0 Release, April, 1996 (L. V. East)

// User interface improvements, additional data integrity tests and additional

// calculational capabilities as outlined in INEL/RWMC system Change Request

Title: SWEPP Assay system

Project ID: Task Baseline Agreement SDM-92-097

Developed by: Scientific Computing Unit, INEL

Funded by: Transuranic Waste Programs Unit, INEI

1.2 Software Module

\$Workfile: assaysys.h $\$$

Assay system is the application object. Microsoft Windows runs the application as an instance of this object.

. Interface Description

The normal application object methods are provided to create, destroy and initialize an instance of the object. 
// No. 13623 (February, 1996). See Software Requirements Specification,

// INEL-96/0056, and Software Design Description, INEL-96/0057, for a detailed

// description of this program.

$1 /$

// Rev 1.0 o5 oct 1993 15:25:34 esm

// Initial revision.

11

5. References

// 5.1 SWEPP Assay system Software Requirements specification,

I/ S. D. Matthews, G. K. Becker, E. S. Marwil, and G.V. Miller,

// EGG-RAAM-10423, EG\&G Idaho, Inc., September, 1993.

5.2 SWEPP Assay System Software Design Description, S. D. Matthews and E. S. Marwil, EGG-RAAM-10424, EG\&G Idaho, Inc., September, 1993.

6. Portability

5.3 SWEPP Assay System Software Test Plan and Report, S. D. Matthews, B. G. Gilber, and E. S. Marwil, EGG-RAAM-10425, EG\&G Idaho, Inc., September, 1993.

\subsection{Hardware:}

IBM 386 PC or compatible machine

National Instruments AT-GPIB controller

Microsoft $\mathrm{C} / \mathrm{C}++$ Version 7.0 (Initial development) Microsoft Visual $C++$ Version 1.5 (Version 2.0 development)

$\begin{array}{ll}/ / & \text { Microsoft Visual C+t } \\ / / & \text { Microsoft Windows } 3.1\end{array}$

\#include <float.h>

\#include <math.h>

\#include "errorbar.h" definition

\#define THRESHOLD

\#if !defined(TRUE)

\#define TRUE 1

\#endif

\#if !defined(FAISE)

\#define FALSE

\#endif

\#define MAX_STATUS_TEXT 64

// Global function definitions.

BOOL check printer ();

int find_drive ( char *);
// Needed by 'include_eb()' function for error_bar FLT_EPSILON * $0.5 f / /$ Value sufficiently small it can be // ignored. 


\section{INEL-96/0057}

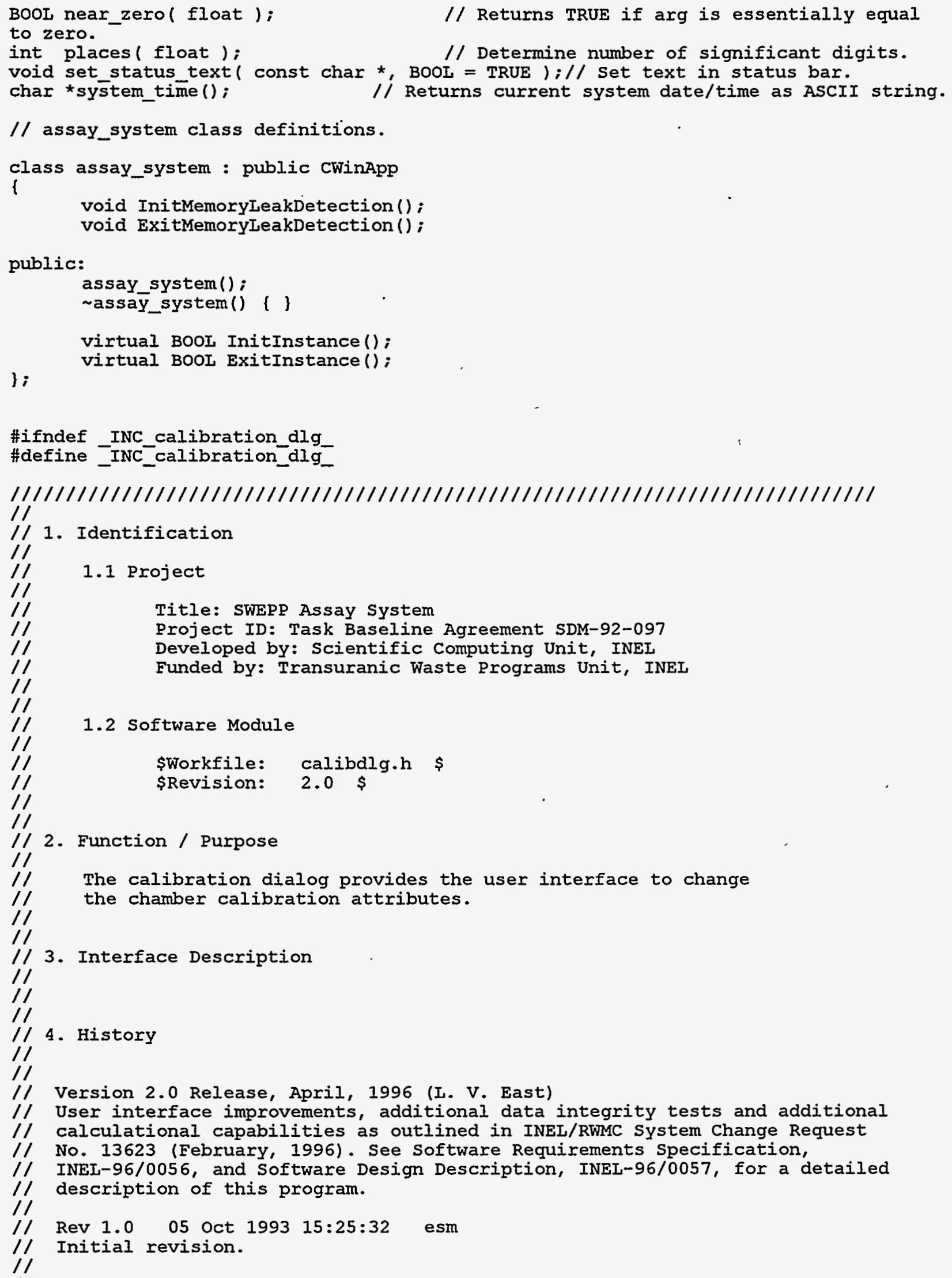

17

// 4. History

1.1 Project

Title: SWEPP Assay system

project ID: Task Baseline Agreement SDM-92-097

Developed by: Scientific Computing Unit, INEL

Funded by: Transuranic Waste Programs Unit, INEL

1.2 Software Module

$\begin{array}{ll}\text { \$Workfile: } & \text { calibdlg.h } \\ \text { \$Revision: } & 2.0 \$\end{array}$

2. Function / Purpose

The calibration dialog provides the user interface to change

the chamber calibration attributes.

3. Interface Description

Version 2.0 Release, April, 1996 (I. V. East)

User interface improvements, additional data integrity tests and additional calculational capabilities as outlined in INEL/RWMC system Change Request No. 13623 (February, 1996). See Software Requirements Specification, INEL-96/0056, and Software Design Description, INEL-96/0057, for a detailed description of this program.

Rev $1.0 \quad 05$ oct $1993 \quad 15: 25: 32$ esm

Initial revision. 
\#include "errorbar.h"

class chamber;

class calibration_dlg : publić cDialog l

chamber *pchamber;

void set_error_bar( error_bar, int, int );

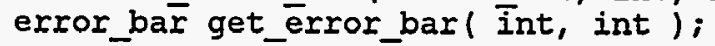

float get_float ( int):

public:

calibration_dlg( chamber * );

calibration_dlg() l;

BOOI OnInitDialog();

I;

void onok();

\#endif

\#ifndef INC_camac_217

\#define INC_camac_217-

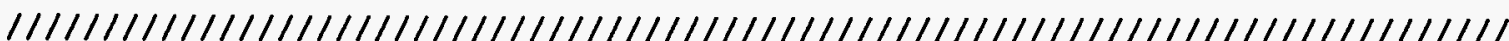
11

// 1. Identification

11

// 1.1 Project

/1

11

Title: SWEPP Assay System

Project ID: Task Baseline Agreement SDM-92-097

Developed by: Scientific Computing Unit, INEL

Funded by: Transuranic Waste Programs Unit, INEL

11

11

II

$1 /$

11

$1 /$

$1 /$

11

$1 /$

2. Function / Purpose

1.2 Software Module

\$Workfile: cam217.h \$

\$Revision: 2.0 \$

$1 /$

// 3. Interface Description

$1 /$

11

$1 /$

4. History

$1 /$

// Version 2.0 Release, April, 1996 (L. V. East)

// User interface improvements, additional data integrity tests and additional

// calculational capabilities as outlined in INEL/RWMC system Change Request

// No. 13623 (February, 1996). See Software Requirements specification,

// INEL-96/0056, and Software Design Description, INEI-96/0057, for a detailed

$/ /$ description of this program. 


\section{INEL-96/0057}

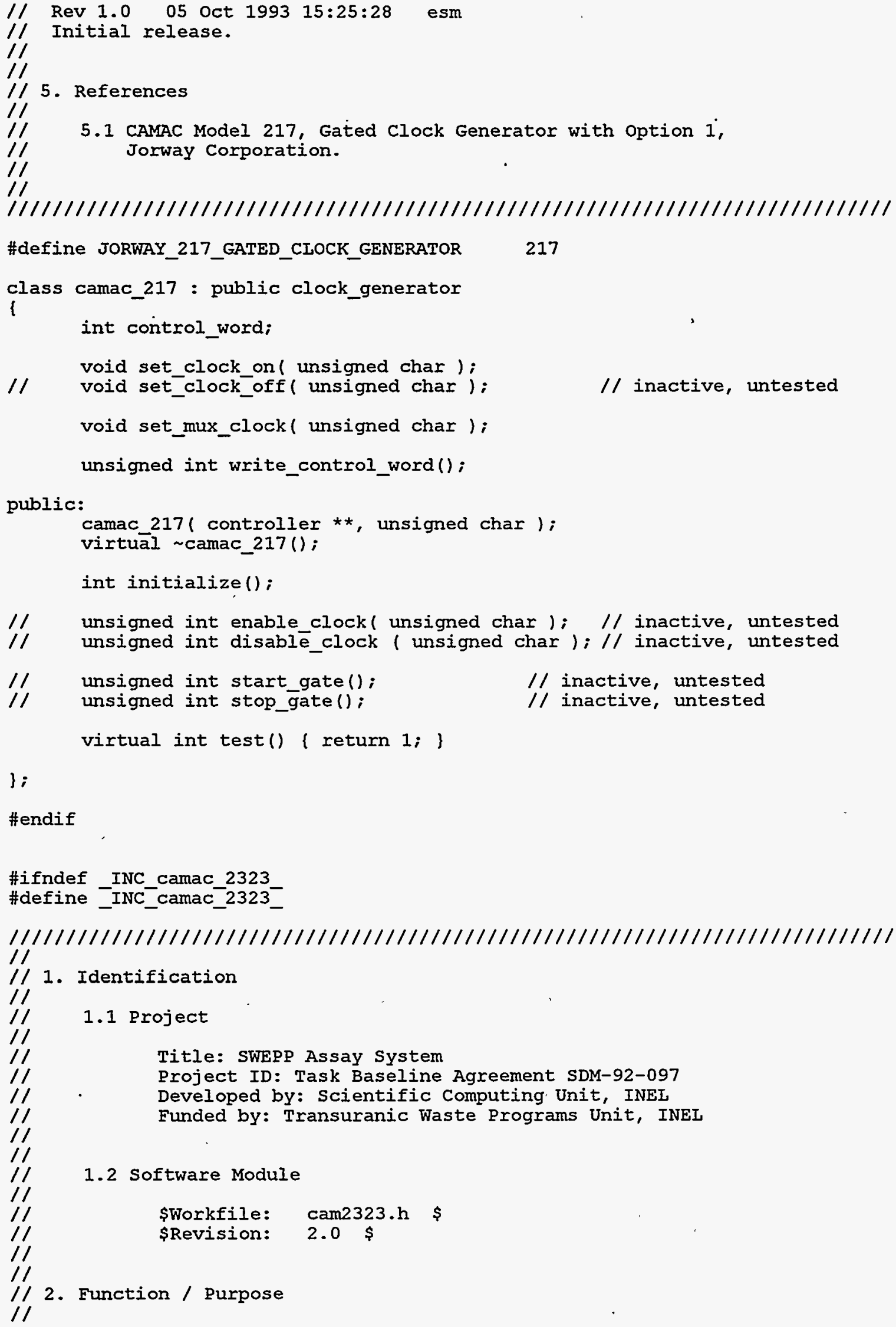

Title: SwEPP Assay System

Project ID: Task Baseline Agreement SDM-92-097

// . Developed by: Scientific Computing Unit, INEL

// Funded by: Transuranic Waste Programs Unit, INEI

// 2. Function / purpose

1.2 Software Module

$$
\text { \$Workfile: cam2323.h \$ }
$$$$
\text { \$Revision: } 2.0 \text { \$ }
$$ 
I/ The CAMAC 2323 models the LeCroy 2323 Dual Gate and Delay Generator.

$1 /$

11

// 3. Interface Description

11

II

$1 /$

// 4. History

II

11

// Version 2.0 Release, April, 1996 (I. V. East)

// User interface improvements, additional data integrity tests and additional

// calculational capabilities as outlined in INEL/RWMC system Change Request

// No. 13623 (February, 1996). See Software Requirements Specification,

// INEL-96/0056, and Software Design Description, INEL-96/0057, for a detailed

// description of this program.

/I

// Rev 1.0 05 Oct 1993 15:25:26 esm

// Initial release.

11

/I

// 5. References

$1 /$

//5 5.1 Operator's Manual, CAMAC Model 2323A, Dual Gate and Delay Generator

// Lecroy, May 1987.

11

11

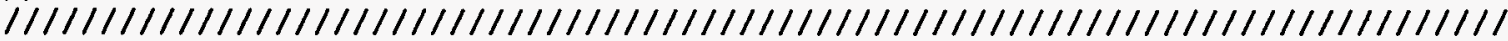

\#define IECROY_2323_DUAI_GATE_GENERATOR2323

class camac_2323 : public gate_generator

1

int write_channel( unsigned char);

// unsigned int read_channel ( unsigned char ); // inactive, untested

// void set_gate_port ( unsigned char ); // inactive, untested virtual void set_gate_mode ( unsigned char, unsigned char );

virtual unsigned char get_gate_mode ( unsigned char );

public:

camac 2323( controller **, unsigned char);

virtuāl camac_2323();

virtual int initialize();

virtual void read_data ( itstream *);

unsigned int start ( unsigned char );

unsigned int stop( unsigned char);

j

virtual int test() \{ return 1; \}

\#endif

\#ifndef INC camac 2551

\#define INC_camac_2551_

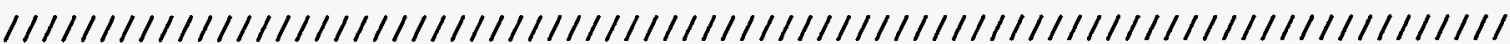

$1 /$

// 1. Identification

$1 /$

$1 /$

$1 /$

$1 /$

1.1 Project

Title: SWEPP Assay System

Project ID: Task Baseline Agreement SDM-92-097 


\section{INEL-96/0057}

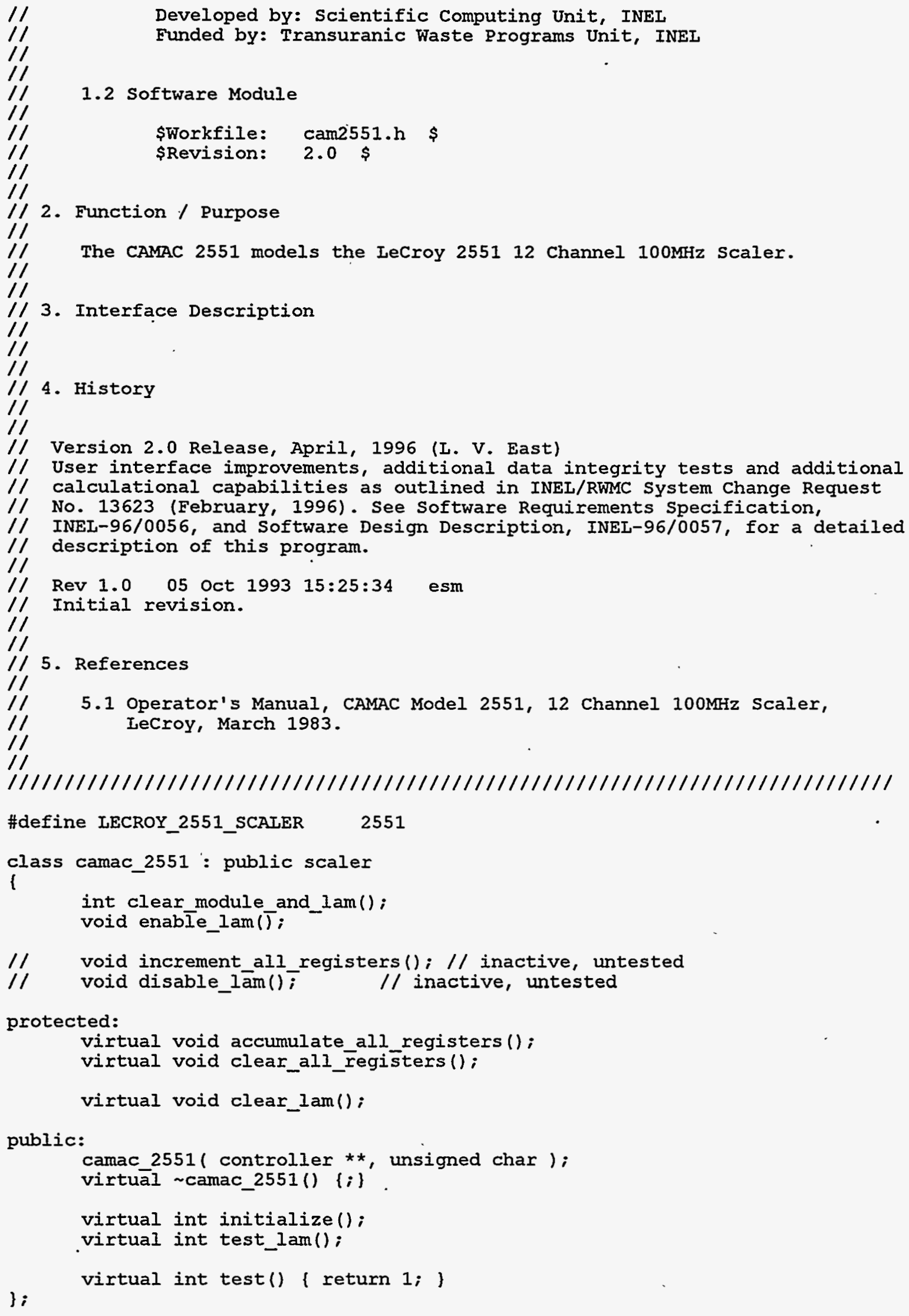


\#endif

\#ifndef INC_camac 450

\#define INC_camac_450

$1 / 1 / 1 / 1 / 1 / 1 / 1 / 1 / 1 / 1 / 1 / 1 / 1 / 1 / 1 / 1 / 1 / 1 / 1 / 1 / 1 / 1 / 1 / 1 / 1 / 1 / 1 / 1 / 1 / 1 / 1 / 1 / 1 / 1$

$1 /$

// 1. Identification

11

$1 /$

$1 /$

11

11

1.0 05 Oct $1993 \quad 15: 25: 26$ esm

Initial release.

Title: SWEPP Assay system

Project ID: Task Baseline Agreement SDM-92-097

Developed by: Scientific Computing Unit, INEI

Funded by: Transuranic Waste Programs Unit, INEI

\subsection{Software Module}

\$Workfile: cam450.h \$

SRevision: $2.0 \$$

2. Function / Purpose

The CAMAC 450 models the DSP 2551 Model QS-450, 100MHz Quad scaler.

3. Interface Description

4. History

Version 2.0 Release, April, 1996 (I. V. East)

User interface improvements, additional data integrity tests and additional calculational capabilities as outlined in INEL/RWMC system Change Request No. 13623 (February, 1996). See Software Requirements Specification, INEL-96/0056, and Software Design Description, INEL-96/0057, for a detailed description of this program.

5. References

$1 /$

5.1 Operator's Manual, CAMAC Model QS-450, 100MHz Quad Scaler, DSP.

11

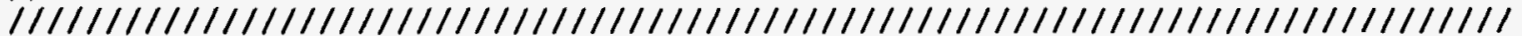

\#define DSP_450_SCALER '450

class camac_450 : public scaler

I/ unsigned char read_module_id_number ();

int test register Iam( unsigñed char);

$/ /$ inactive, untested void cleār_register ( unsigned char);

protected:

virtual void accumulate all registers (); virtual void clear_all_registers(); virtual void clear_lam(); 
public:

camac 450 ( controller $\star \star$, unsigned char );

virtuāl $\sim$ camac_450() $\{$; $\}$

virtual int test_lam();

\};

virtual int test () ( return 1; \}

\#endif

\#ifndef INC camac 8901

\#define _INC_camac_8901_

$/ / / / / / / / / / / / / / / / / / / / / / / / / / / / / / / / / / / / / / / / / / / / / / / / / / / / / / / / / / / / 1$

$1 /$

// 1. Identification

$1 /$

$1 /$

$1 /$

II

User interface improvements, additional data integrity tests and additional

// calculational capabilities as outlined in INEL/RWMC system Change Request

// No. 13623 (February, 1996). See Software Requirements specification,

// INEL-96/0056, and Software Design Description, INEL-96/0057, for a detailed

$/ /$ description of this program.

$1 /$

// Rev 1.0 05 Oct $1993 \quad 15: 25: 32$ esm

// Initial release.

$1 /$

$1 /$

// 5. References

$1 /$

$1 /$

11

$/ / / / / / / / / / / / / / / / / / / / / / / / / / / / / / / / / / / / / / / / / / / / / / / / / / / / / / / / / / / / / 1$

\#define IECROY_8901_CONTROLLER 8901 


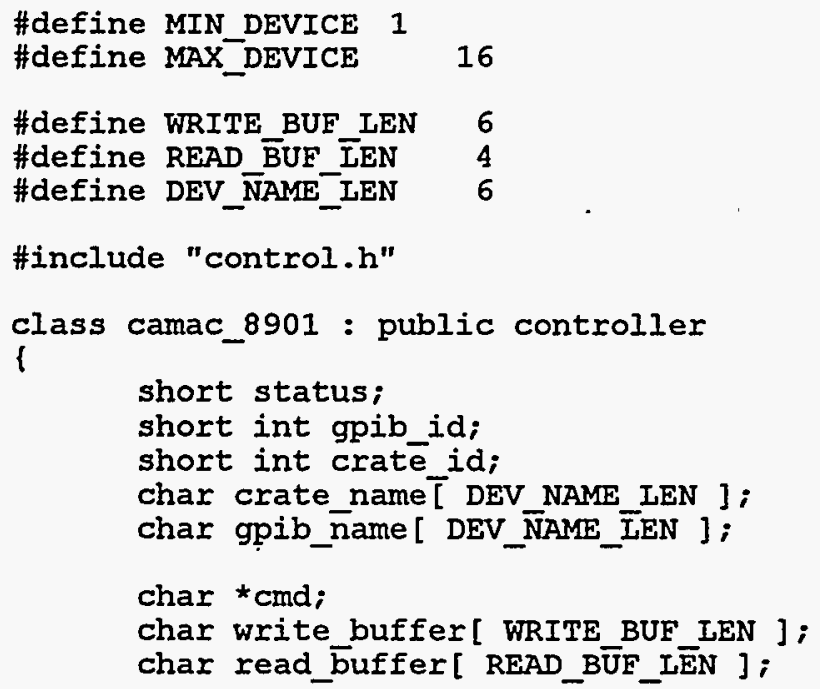




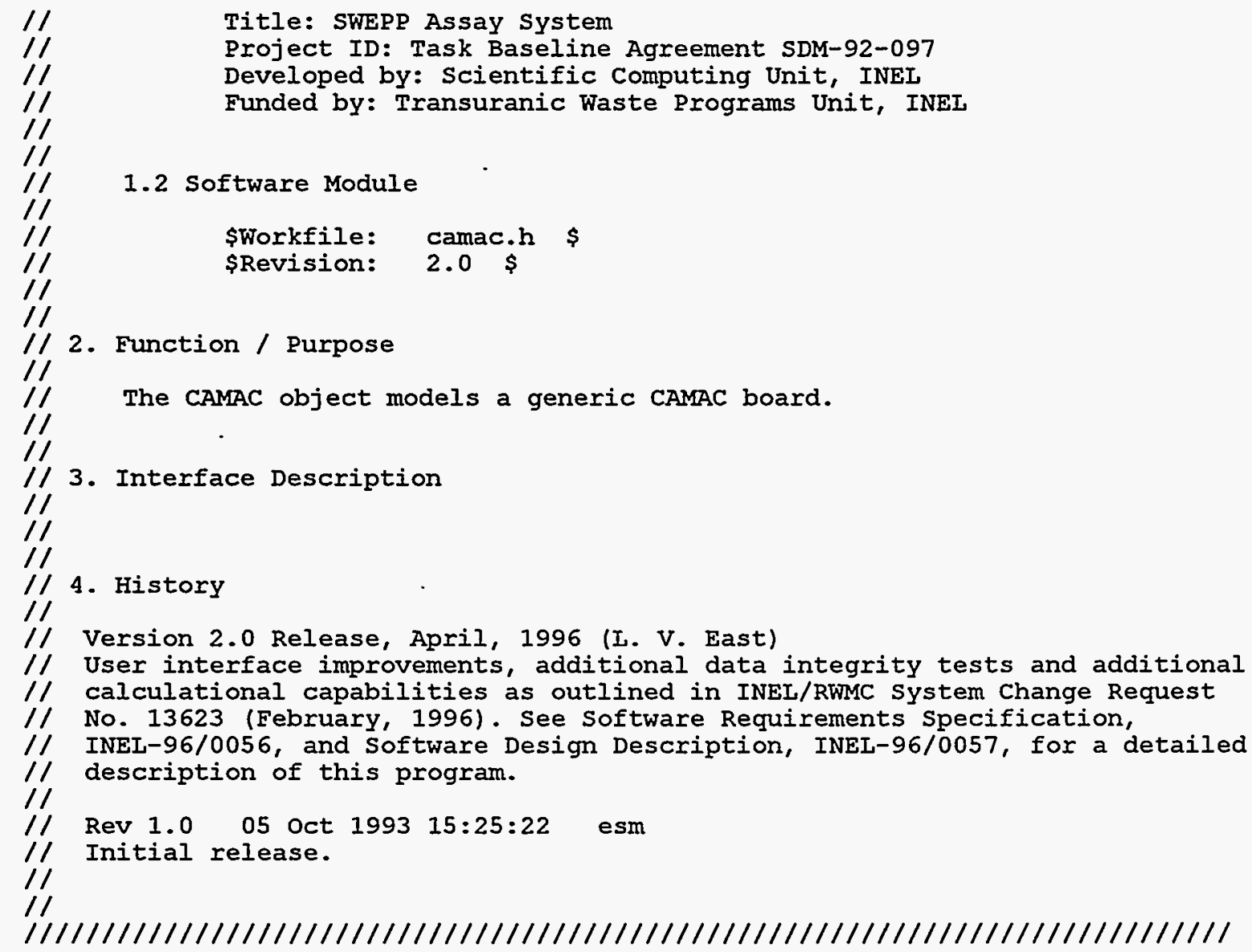

\subsection{Software Module}

2. Function / Purpose

The CAMAC object models a generic CAMAC board.

11

11

11

//IEEE std 583-1975 CAMAC Function Codes

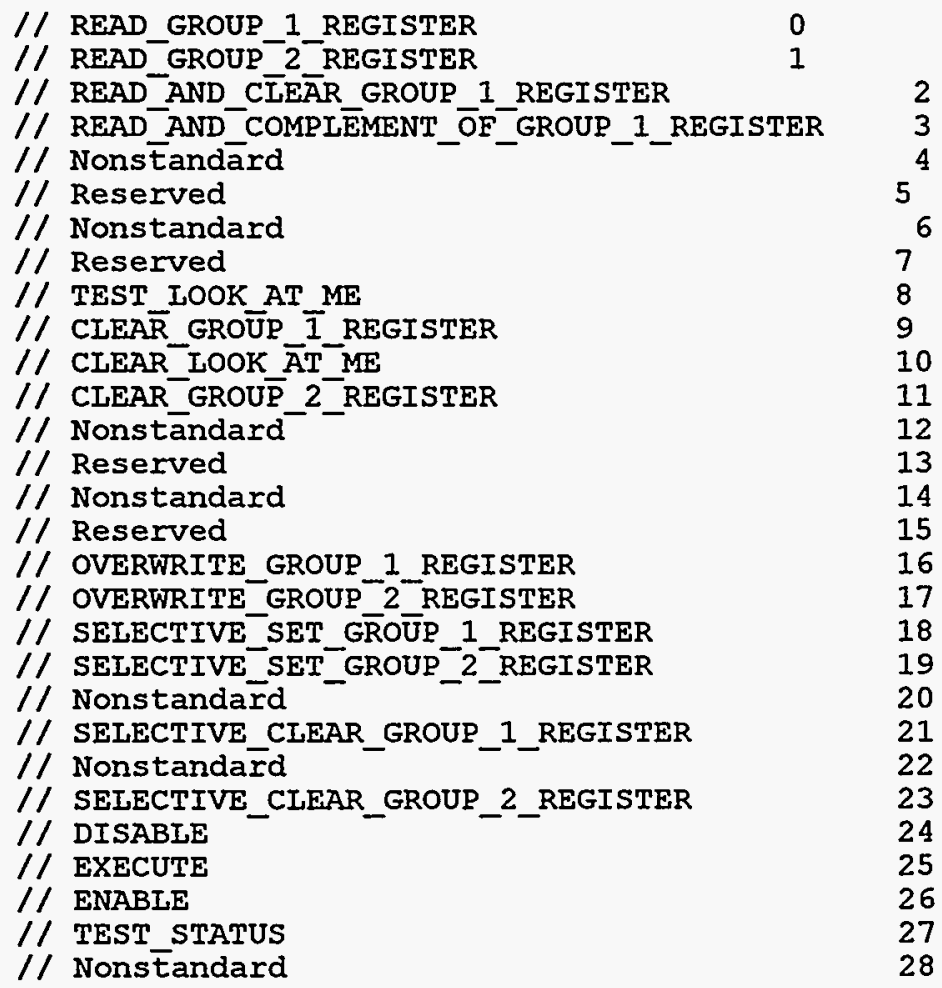




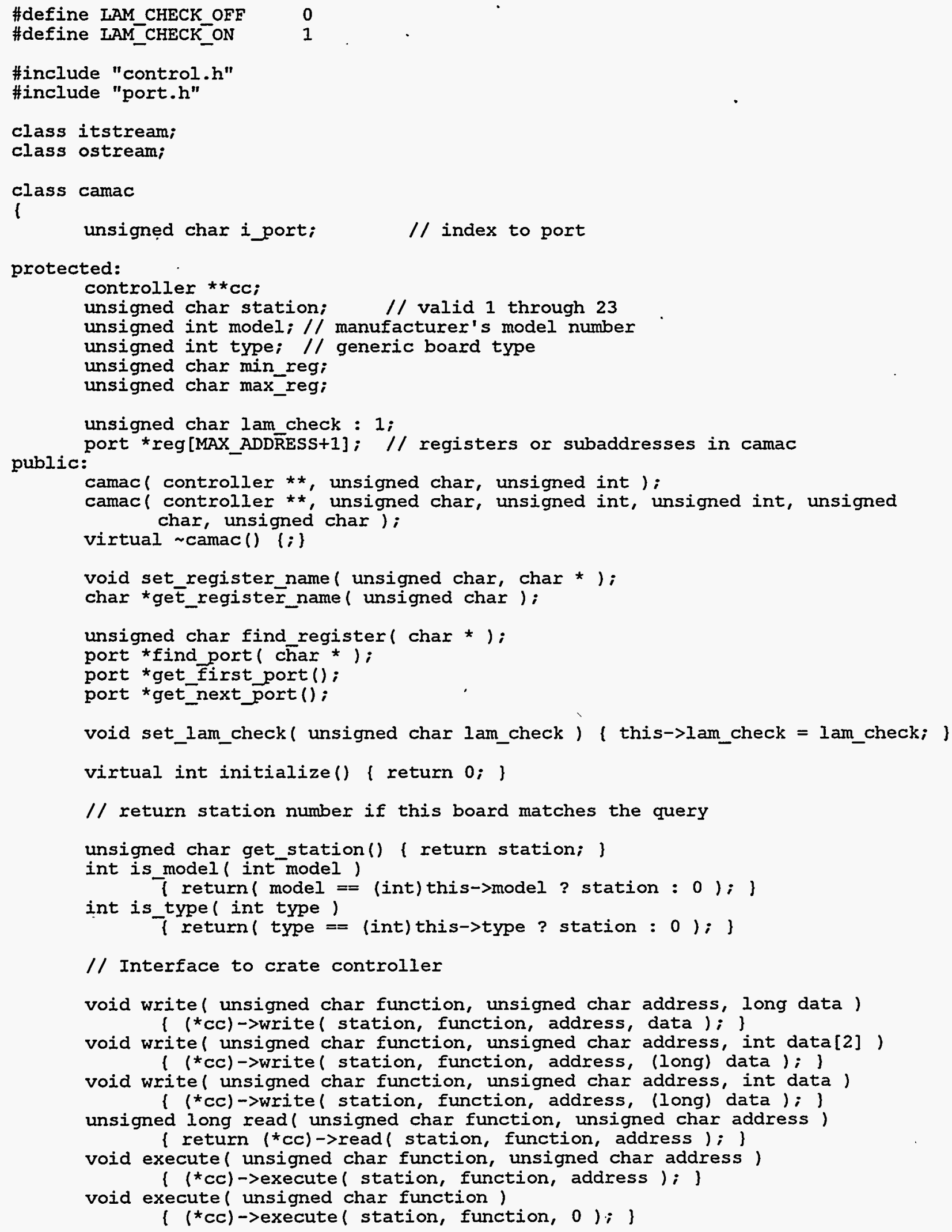




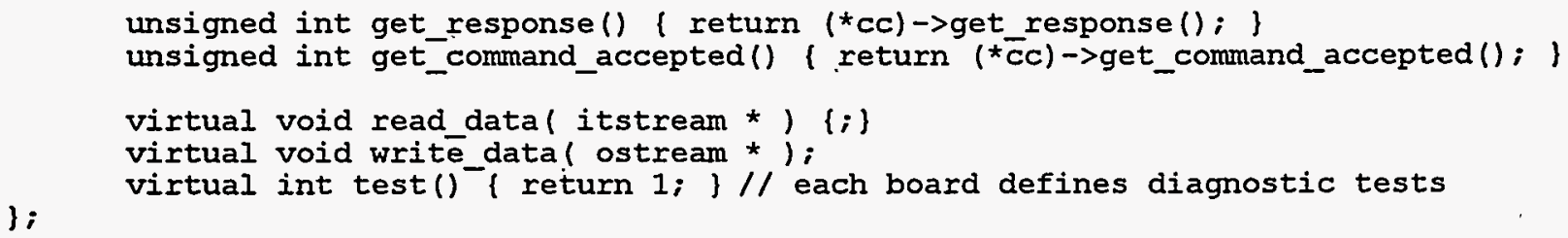




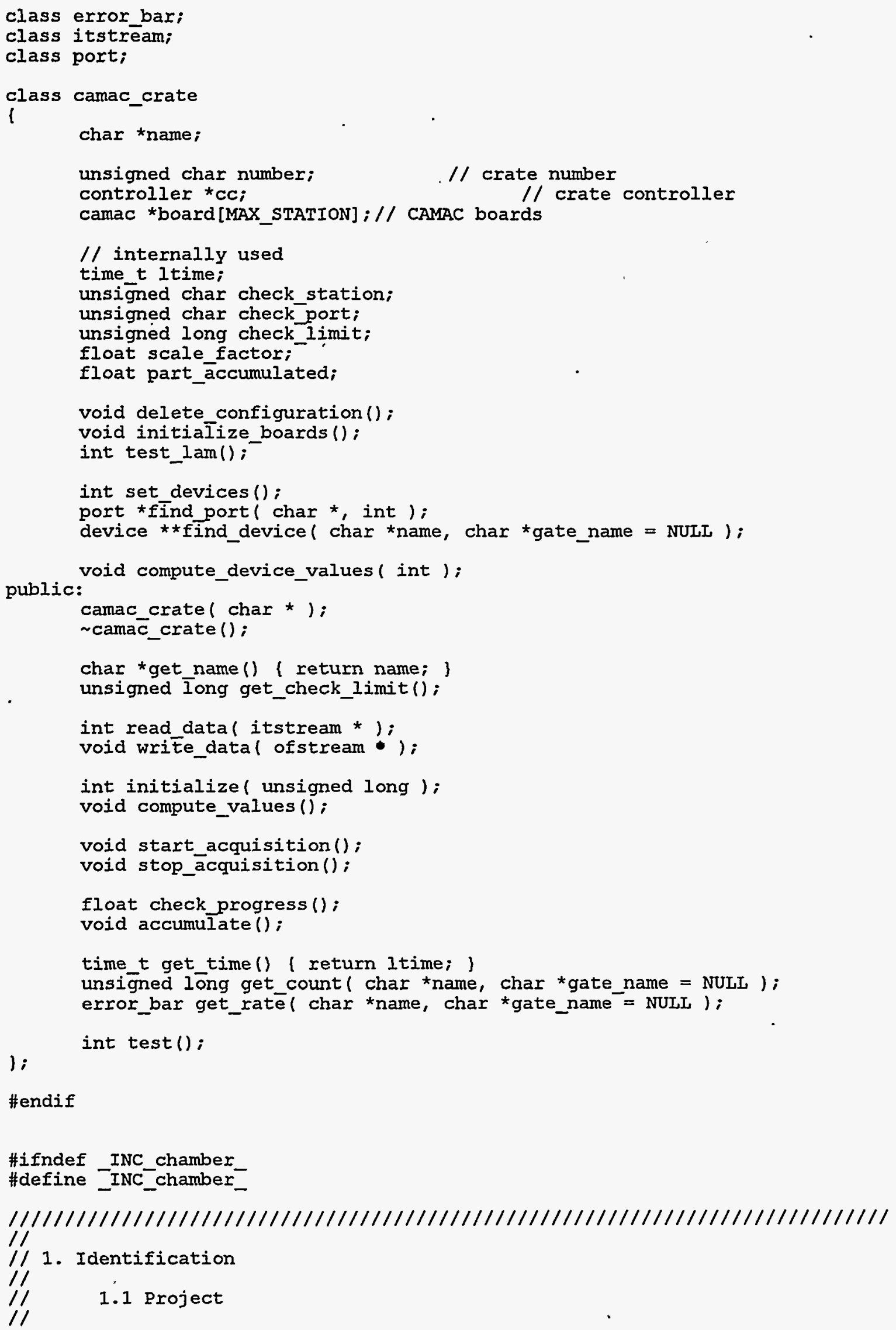


camac_crate *passive_background;

camac crate *passive gross;

camac_crate *pcamac_crate;

public:

active signals *active;

passive signals * passive;

passive_signals *background;

chamber ( char *);

chamber ();

void set file name ( char *);

int can_write() ( return has_write_permission; )

int read,calibration_data ();

int read-calibration data ( itstream *);

void write calibration data ();

void write_calibration_data( ofstream * );

char *get_name() \{ return name; \}

error bar get shielded detector efficiency ()

( return shieldē_detector_efficiency; )

error bar get system detector efficiency()

( return system_detector_efficiency; \}

error_bar get_active_zero_matrix_calibration_factor() ( return active zmcF; \}

error_bar get_shielded̄_coincidence_zero_matrix_calibration_factor() \{ return shielded_coincidence_zmcf; \}

error_bar get_system_coincidence_zero_matrix_calibration_factor() $\{$ return system_coincidence_zmcF; \}

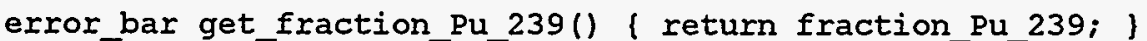

error_bar get_fraction_Pu_240() ( return fraction_Pu_240; \}

time_t get_time() ( return pcamac_crate->get_time(); )

time ${ }^{-}$get active time() ( return active crate->get time (); )

time_t get passive time() ( return passiv̄e gross->get time (); \}

time_t get background time () ( return passive background->get time (); )

char *get crate name () ( return pcamac crate->get name (); )

unsigned Iong gêt check limit() | return pcamac crate->get check limit (); )

int initialize_active ( unsigned long );

int initialize passive ( unsigned long);

int initialize background ( unsigned long);

void start acquisition() ( pcamac crate->start acquisition(): \}

float chec $\bar{k}$ progress () ( return pcamac_crate->check progress (); )

void stop acquisition() ( pcamac crate->stop acquisition(); \}

void accumulate() ( pcamac crate->accumulate ()$;$ \}

void set_background_correction( unsigned int background_correction )

( passive->set_background_correction( background_correction ); \}

int read_active_data ( itstream *data)

\{ return active crate->read data ( data): \}

int read passive_data ( itstream * data)

\{ return passive gross->read data (data); \}

int read background_data ( itstream ${ }^{\star}$ data)

\{ return passive background->read data ( data ); \}

void compute active values()

\{ active crate->compute values (); \}

void compute passive_values()

l

passive_gross->compute_values () ; 
INEL-96/0057

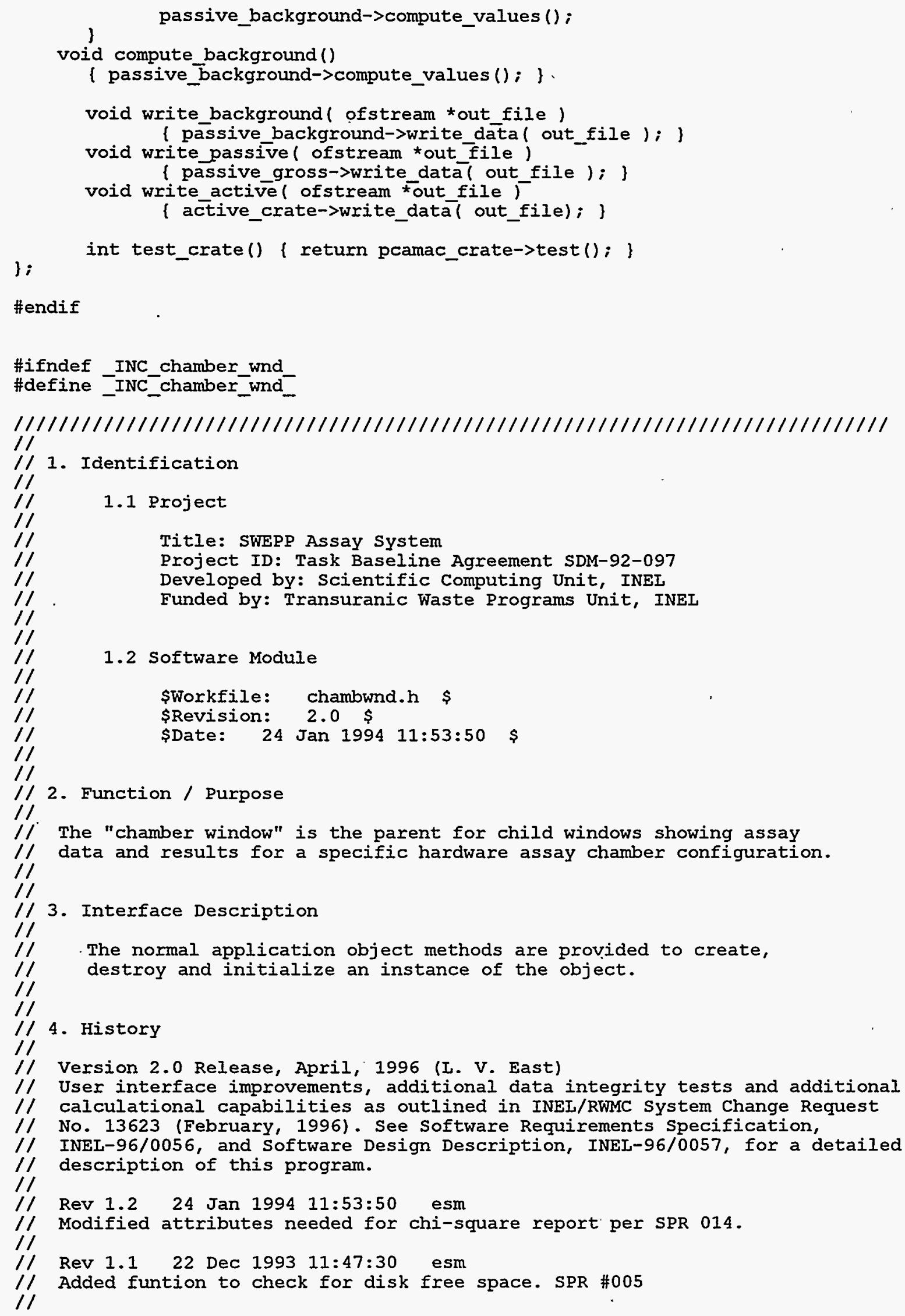

//2. Function / Purpose

// The "chamber window" is the parent for child windows showing assay

// data and results for a specific hardware assay chamber configuration.

II

\section{Interface Description}

The normal application object methods are provided to create,

// 4. History

II

// Version 2.0 Release, April, 1996 (L. V. East)

// User interface improvements, additional data integrity tests and additional // calculational capabilities as outlined in INEL/RWMC system Change Request

// No. 13623 (February, 1996). See Software Requirements Specification,

// INEL-96/0056, and Software Design Description, INEL-96/0057, for a detailed

// description of this program.

II

// Rev 1.2 24 Jan 1994 11:53:50 esm

// Modified attributes needed for chi-square report per SPR 014 .

$1 /$

// Rev 1.1 22 Dec 1993 11:47:30 esm

// Added funtion to check for disk free space. SPR \#005

II 
// Rev 1.0 05 oct $1993 \quad 15: 25: 30$ esm

// Initial release.

$1 /$

11

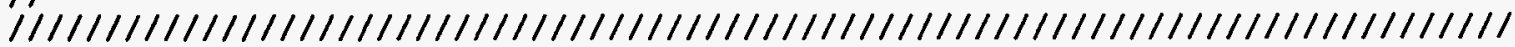

\#include <fstream.h>

\#include <stdlib.h>

\#include "errorbar.h"

// Private message definition -- used to notify waste_dlg of a menu item change. \#define STATUS_CHANGED (WM_USER + 501)

// auto state values

\#define AUTO_OFF 0

\#define AUTO-RUN 1

\#define AUTO_PAUSE - 1

class auto dlg;

class chamber;

class container;

class dms dlg;

class error bar;

class itstream;

class matrix;

class chamber_wnd : public CMDIChildwnd

\}

friend class process_options_dlg;

friend class waste_dig;

friend class active dlg;

friend class passive_dlg;

friend class results_dlg;

static dms dlg *pdms dlg;

static autō_dlg *paut̄o,dIg;

static BOOL NEAR is remote operation:

static int NEAR auto mode_state;

static int NGAR chamber count;

static int NEAR arch_flagg;

// Number of active chamber windows. // Last archive drive requested.

CWnd *parent_wnd;

chamber * pchamber;

matrix * content;

itstream *ac_ini;

unsigned long bkg_secs;

unsigned long num pulses;

unsigned long num secs;

unsigned long ver pulses;

unsigned long ver_secs;

unsigned long pulse rate;
int passive delay;
// Delay before starting passive after active assay.
int last_code;
// Most receltly used content code.
BOOL active_first;
// Determines order of active \& passive data
// acquisition.
BOOL auto save;
// If TRUE, automatically save data after an assay.
BOOL backğround correction; // Passive background correction flag.
BOOL good background; // TRUE only if background data-read successful.
BOOL inclūde U233; // If TRUE, report U-233 mass (bassed in mass ratio info)
BOOL include-U235; // If TRUE, report U-235 mass (bassed in mass ratio info)
BOOL include_Pu242; // If TRUE, report $\mathrm{Pu}-242$ mass.
BOOL summary printed; // TRUE if summary report printed. 
INEL-96/0057

BOOI summary_report;

$/ /$ Print ON/OFE flag.

COLORREF fill_color;

cstring str_profile_heading;

char file_name[_MAX_FNAME];

char last_file_name[_MAX_FNAME];

char *pat $\bar{h}$ name;

char CW_dir̄[_MAX_PATH]; // Current default directory for this chamber.

int cw_drive; / / Current default disk drive for this chamber.

char container ID[32]; // ID applied to waste container by waste generator.

char arckive_ID $[16]$;

// Administrative label for archive diskette.

waste_dlg *pwaste_dlg;

active dlg tpactive dlg;

passive_dlg *ppassiv̄e_dlg;

results_dlg *presults_dlg;

$1 /$ directories

char * root_dir;

int root_drive;

char *arch dir;

int arch_drive;

char *gamma_dir;

char *backg-dir;

char *verif_dir;

char *calib-dir;

char *prod_dir;

// files

char *options_name;

char *backg_name;

char *verif_name;

char *passivie_cfg;

char *active_cfg;

char * correl_dat;

char *chi sq_dat;

char *nuclide dat;

char *default_mass_dat;

char * gamma dāt;

char * regress_dat;

// other stuff...

int state;

BOOL bad_data;

// See chambwnd.cpp for "state" definitions.

recalc.

BOOL is_file_saved;

// TRUE if bad data discovered during assay or

BOOL is active done;

BOOL is passive done;

BOOL is_background_done;

BOOL is_verification;

unsigned int chi_square_sample_size;

unsigned int chi_square_count Eime;

float chi square acceptance min;

float chi-square acceptance max;

float chi-square confidence $\bar{i}$

float chi-sq shielded total;

float chi_sq_system_total;

unsigned long *shielded total;

unsigned long *system_tōtal; 


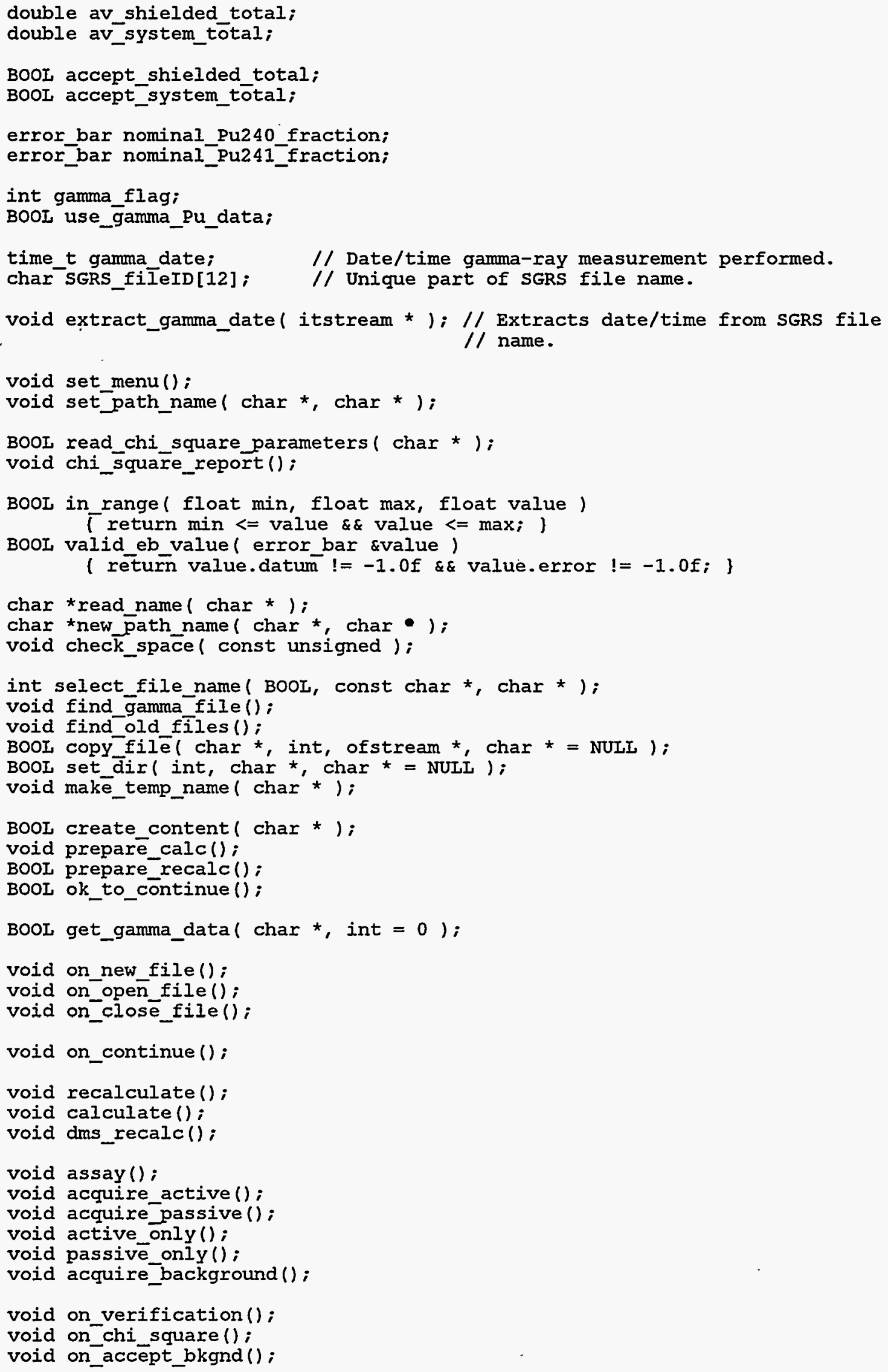




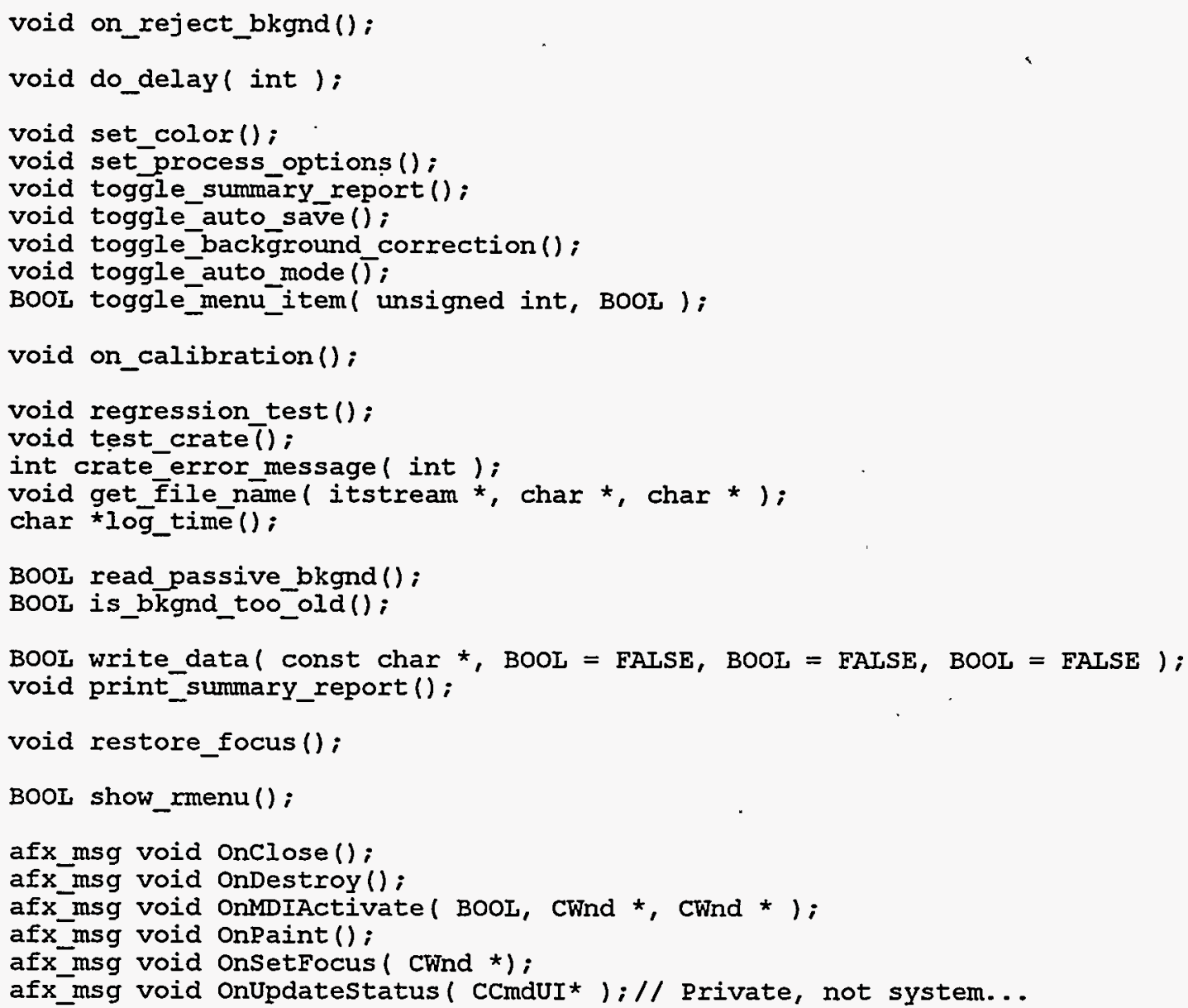




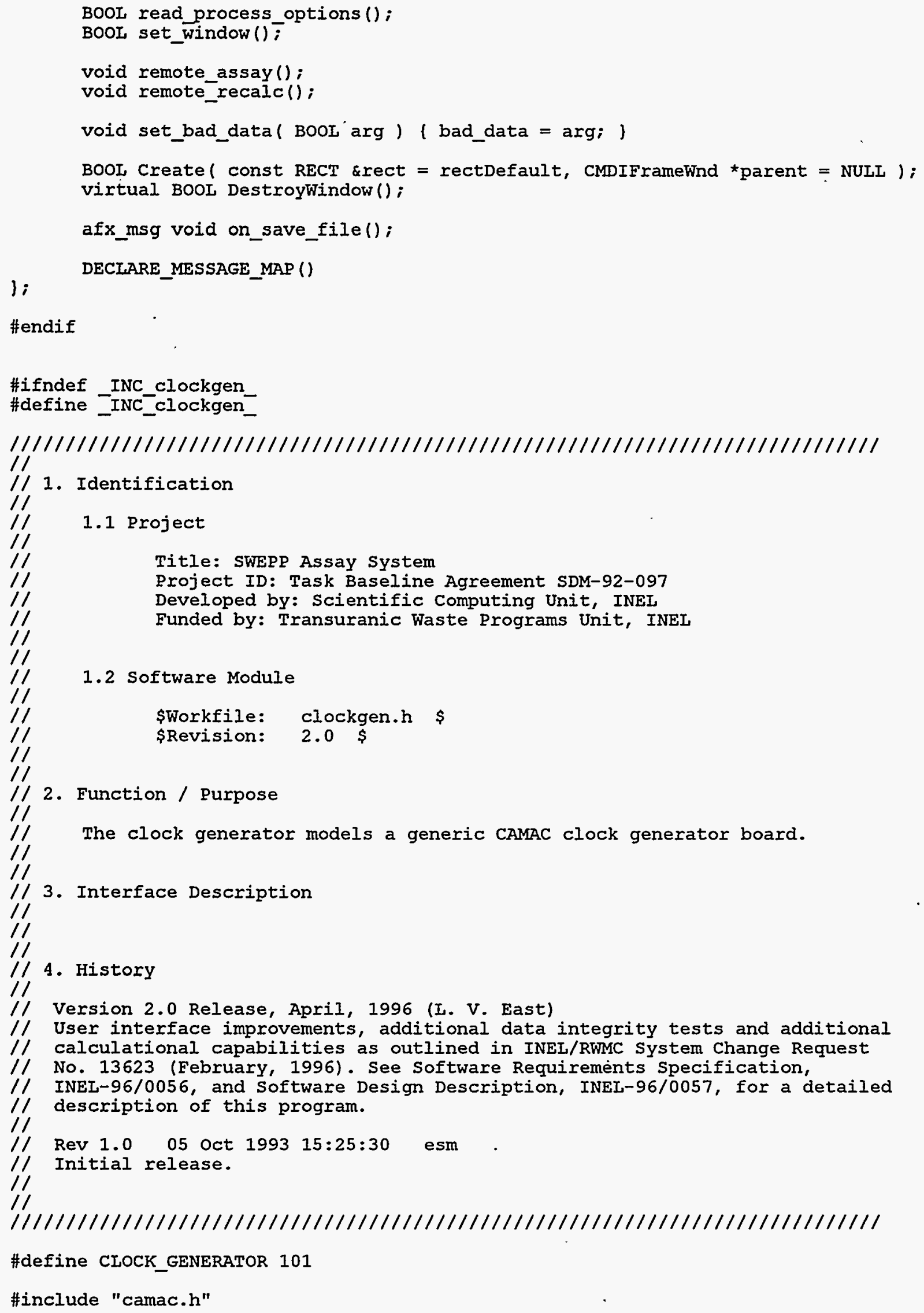




\section{INEL-96/0057}

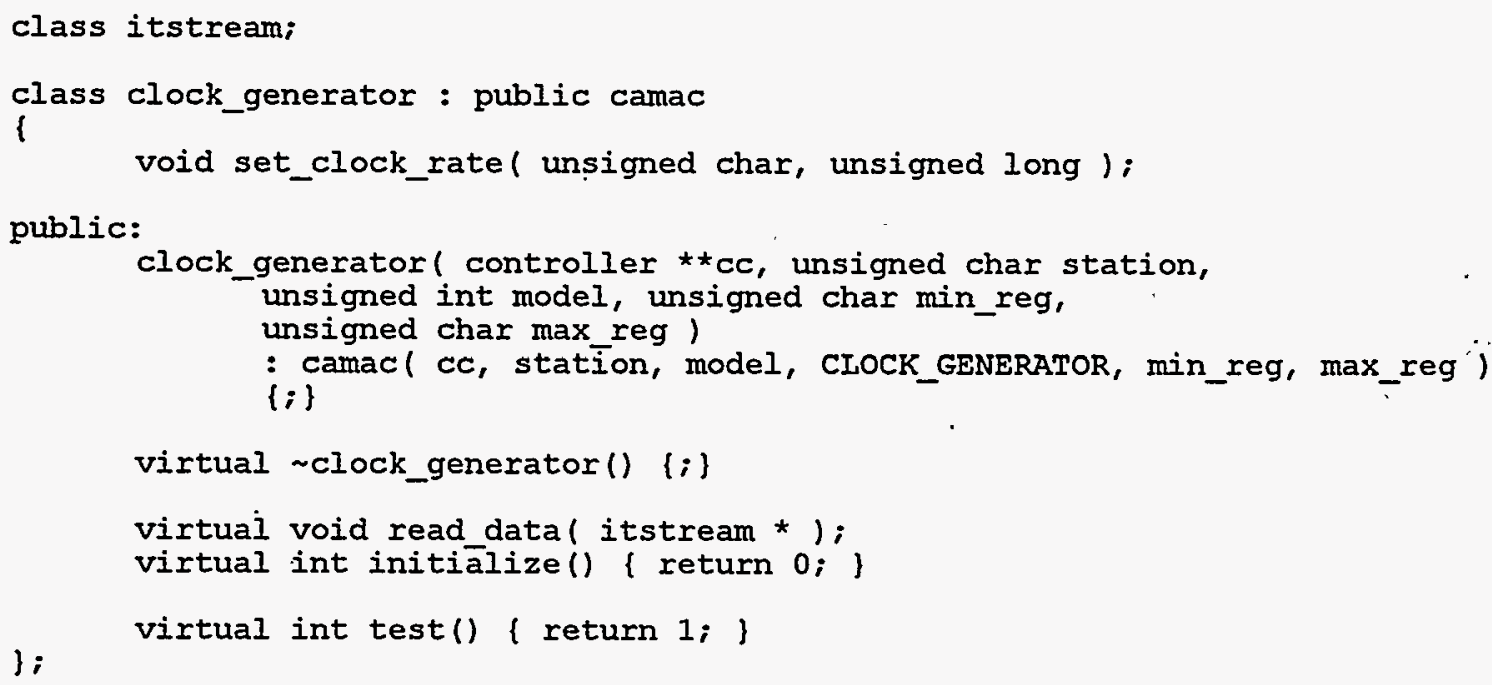

// 4. History 


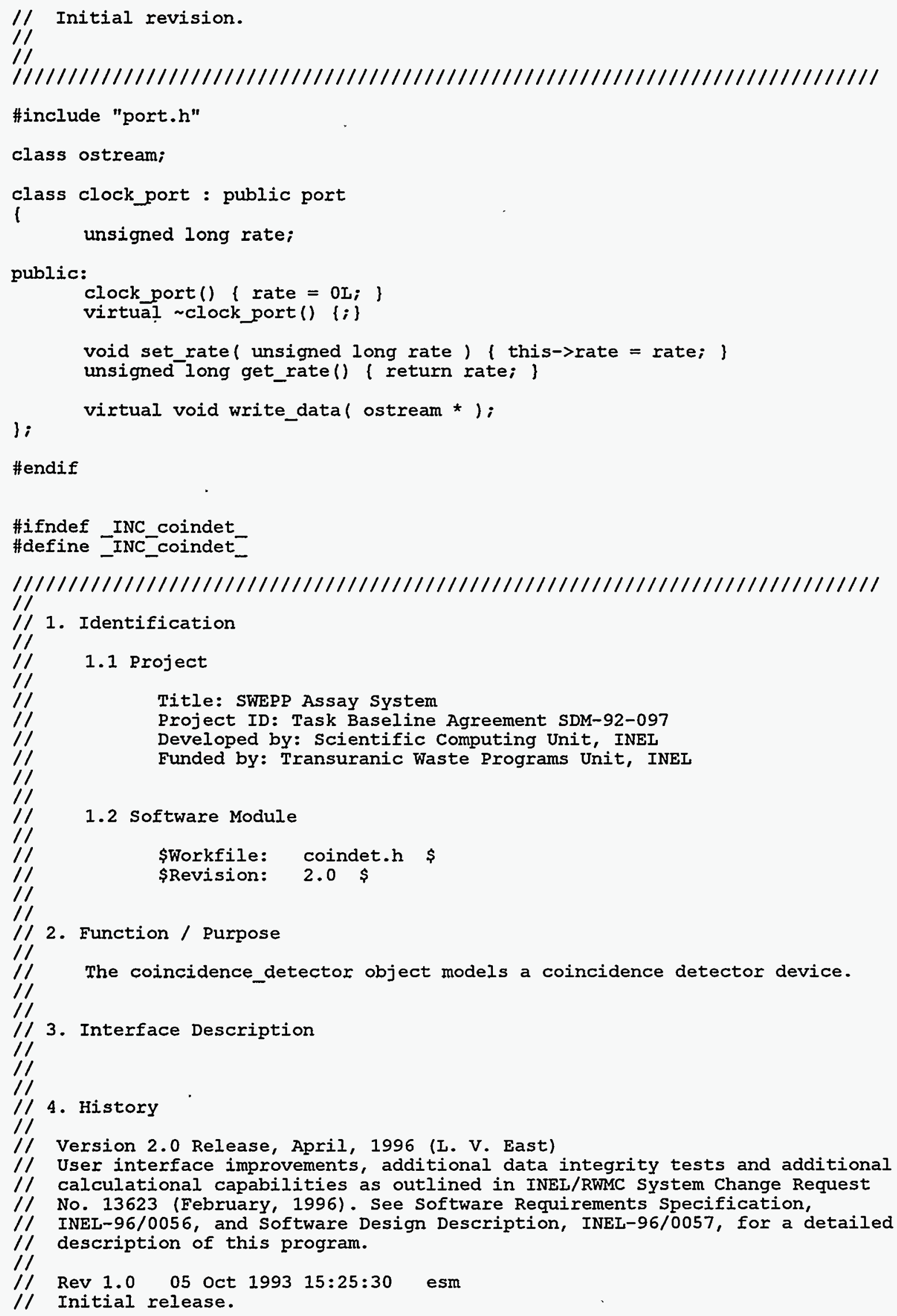

// 1.2 Software Module

// 2. Function / Purpose 
INEL-96/0057

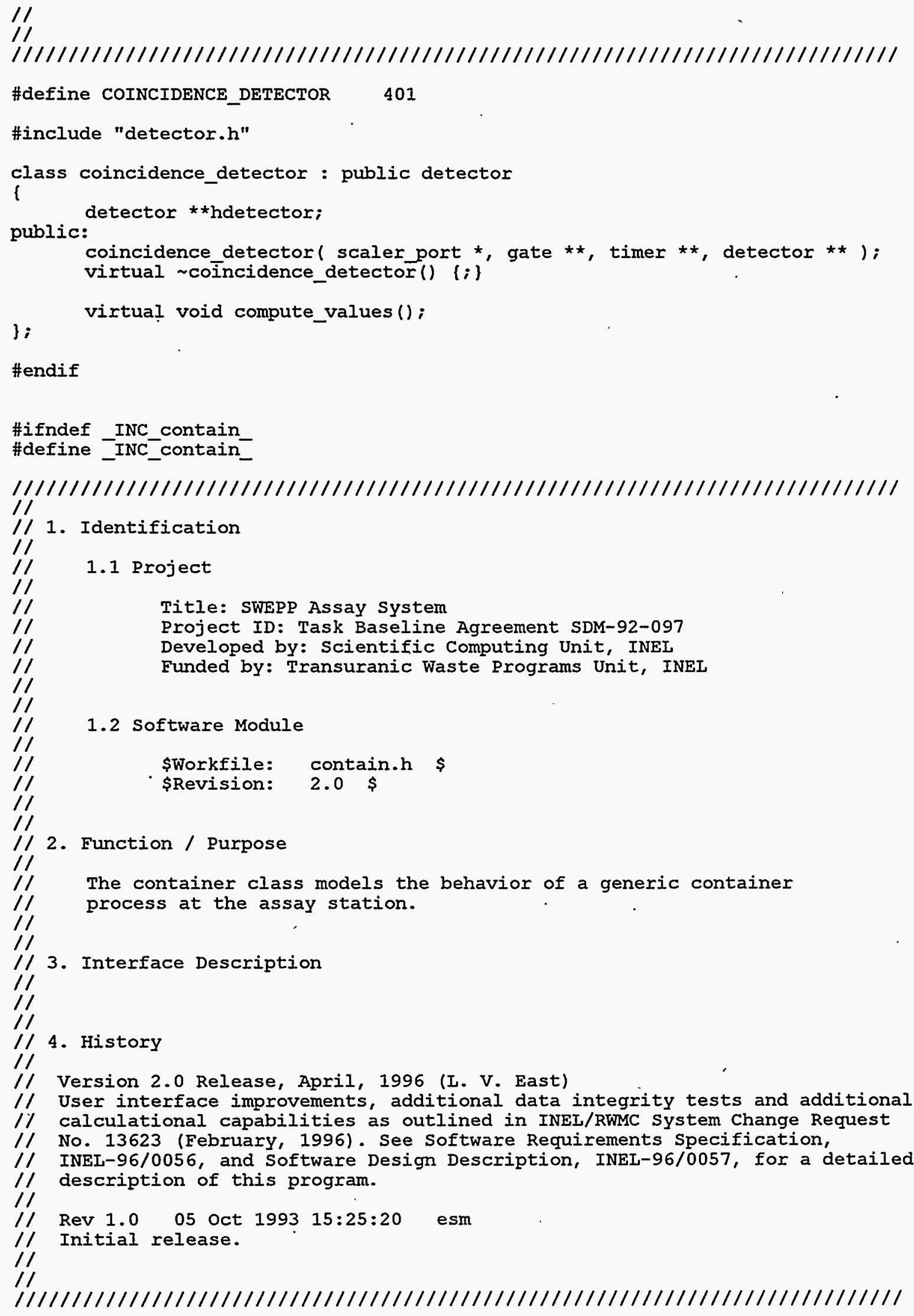




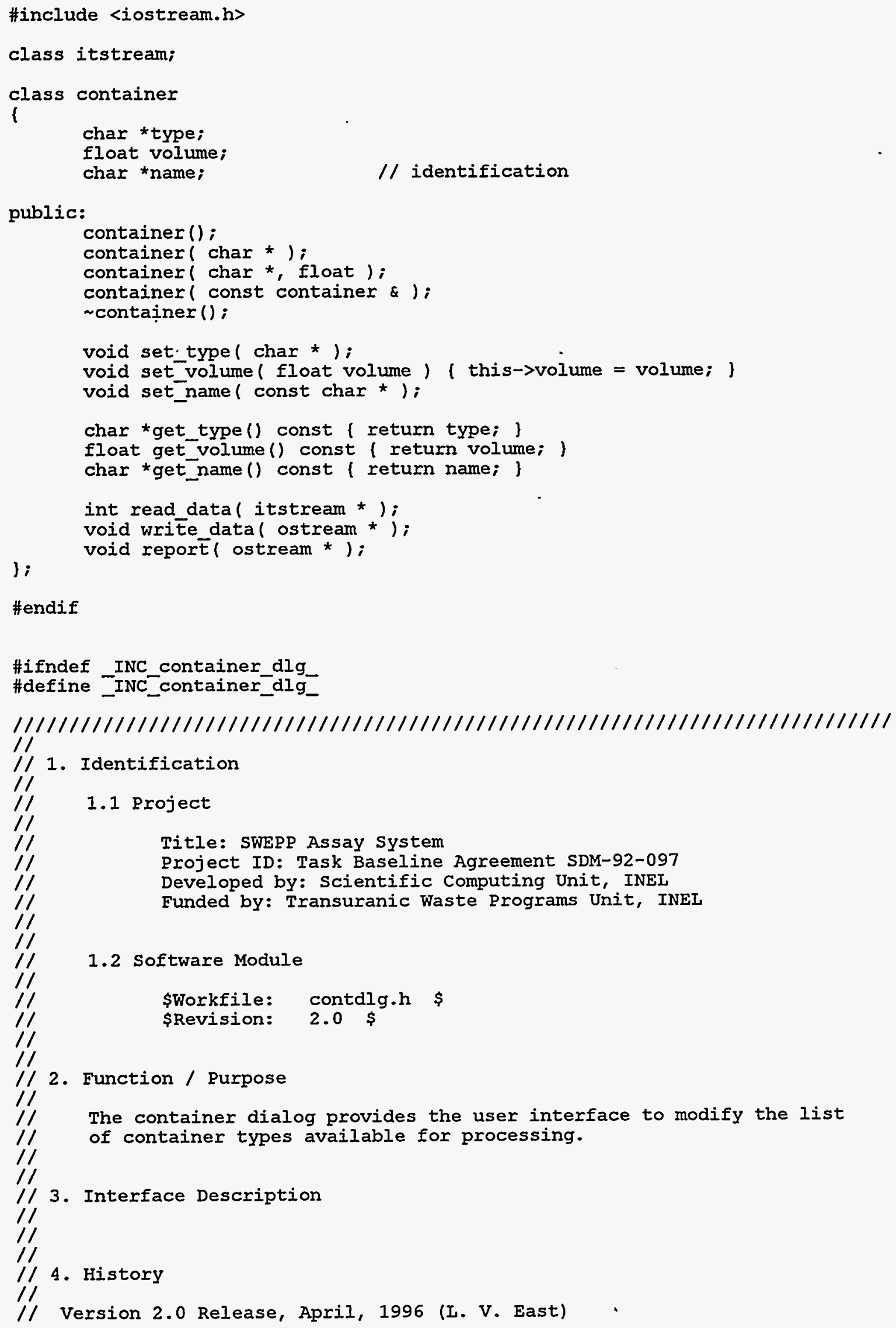

\#endif

\#ifndef INC container dlg

\#define - INC_container_dlg_

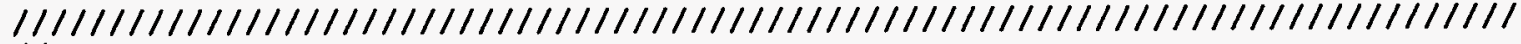

$1 /$

// 1. Identification

$1 /$

// 1.1 Project

11

// 2. Function / Purpose

// 3. Interface Description

The container dialog provides the user interface to modify the list of container types available for processing.

$1 /$

$1 /$

// 4. History

11

// Version 2.0 Release, April, 1996 (L. V. East)

Title: SWEPP Assay system

Project ID: Task Baseline Agreement SDM-92-097

Developed by: Scientific Computing Unit, INEI

Funded by: Transuranic Waste Programs Unit, INEL

1.2 Software Module

\$Workfile: contdlg.h \$

\$Revision: 2.0 \$ 


\section{INEL-96/0057}

// User interface improvements, additional data integrity tests and additional // calculational capabilities as outlined in INEL/RWMC system Change Request

// No. 13623 (February, 1996). See Software Requirements Specification,

// INEL-96/0056, and Software Design Description, INEL-96/0057, for a detailed

// description of this program.

$/$ Rev 1.0 oct $1993 \quad 15: 25: 32$ esm

// Initial release.

11

$1 /$

$1 / 1 / 1 / 1 / 1 / 1 / 1 / 1 / 1 / 1 / 1 / 1 / 1 / 1 / 1 / 1 / 1 / 1 / 1 / 1 / 1 / 1 / 1 / 1 / 1 / 1 / 1 / 1 / 1 / 1 / 1$

class container;

class container_dlg : public cDialog

l

char $\star$ title;

char *text;

float volume;

container *pcontainer;

int sel;

BOOL changed; $\quad / /$ Flag to indicate whether list has been changed.

BOOI can_write; // TRUE if underlying file is write enabled.

CListBox *list_box;

BOOI get_dlg_data ( int);

void set dlg data ();

BOOL is duplicate();

void set button state () ;

void resêt_focus ( int);

afx msg void on add ();

afx_msg void on change ();

afx msg void on delete();

afx_msg void on selchange ();

afx-msg void on $\bar{P}$ aint ();

public:

container dlg();

containe $\bar{r}$ dlg () ;

BOOL OnInitDialog();

void onok();

void oncancel ():

\} ;

DECLARE_MESSAGE_MAP();

\#endif

\#ifndef INC_container_list

\#define INC_container_list-

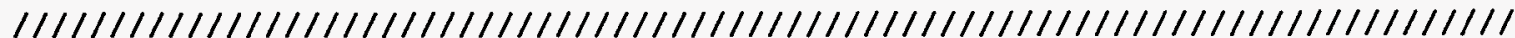

$1 /$

// 1. Identification

$1 /$

$1 /$

$1 /$

$1 /$

1.1 Project

Title: SWEPP Assay system

$\begin{array}{ll}/ / & \text { Project ID: Task Baseline Agreement SDM-92-097 } \\ / / & \text { Developed by: Scientific Computing Unit, INEI }\end{array}$ 
// Funded by: Transuranic Waste Programs Unit, INEI

// 2. Function / Purpose

1.2 Software Module

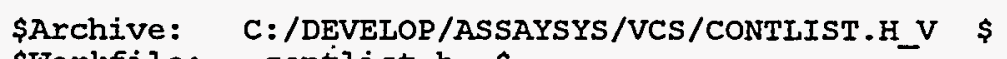

1/ The container_list models the list of containers used at the

// assay station.

11

// 3. Interface Description

$1 /$

// Functions are defined for appending, inserting, and

// removing containers. A find function searches the list for

/1 a container with a specified type.

$1 /$

// 4. History

$1 /$

// Rev 1.0 05 Oct $1993 \quad 15: 25: 32$ esm

//Initial revision.

II

$1 /$

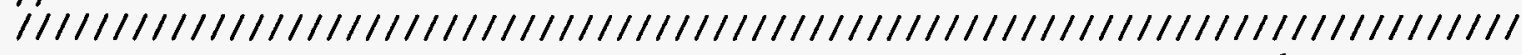

\#include "list.h"

class container;

class container_list : public list

l

void reset();

public:

container list ( char *);

containe $\bar{r}$ list ();

virtual int read data();

virtual void writ̄e_data();

void append ( container *pc) ( list: :append ( (void *) pc ); \}

void insert ( container *pc) ( list: insert ( (void *) pc); )

void remove ( container *pc) ( list: :remove ( (void *) pc); )

container *find ( char *);

container *get_first () ( return (container *) list::get_first (); )

; ; container * get_next () ( return (containex *) list::get_next ();

\#endif

\#ifndef INC controller

\#define _INC_controlier_

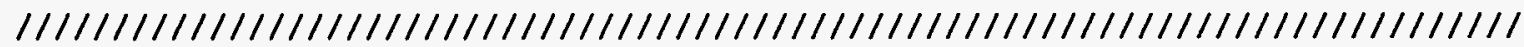

11

// 1. Identification

/l 


\section{INEL-96/0057}

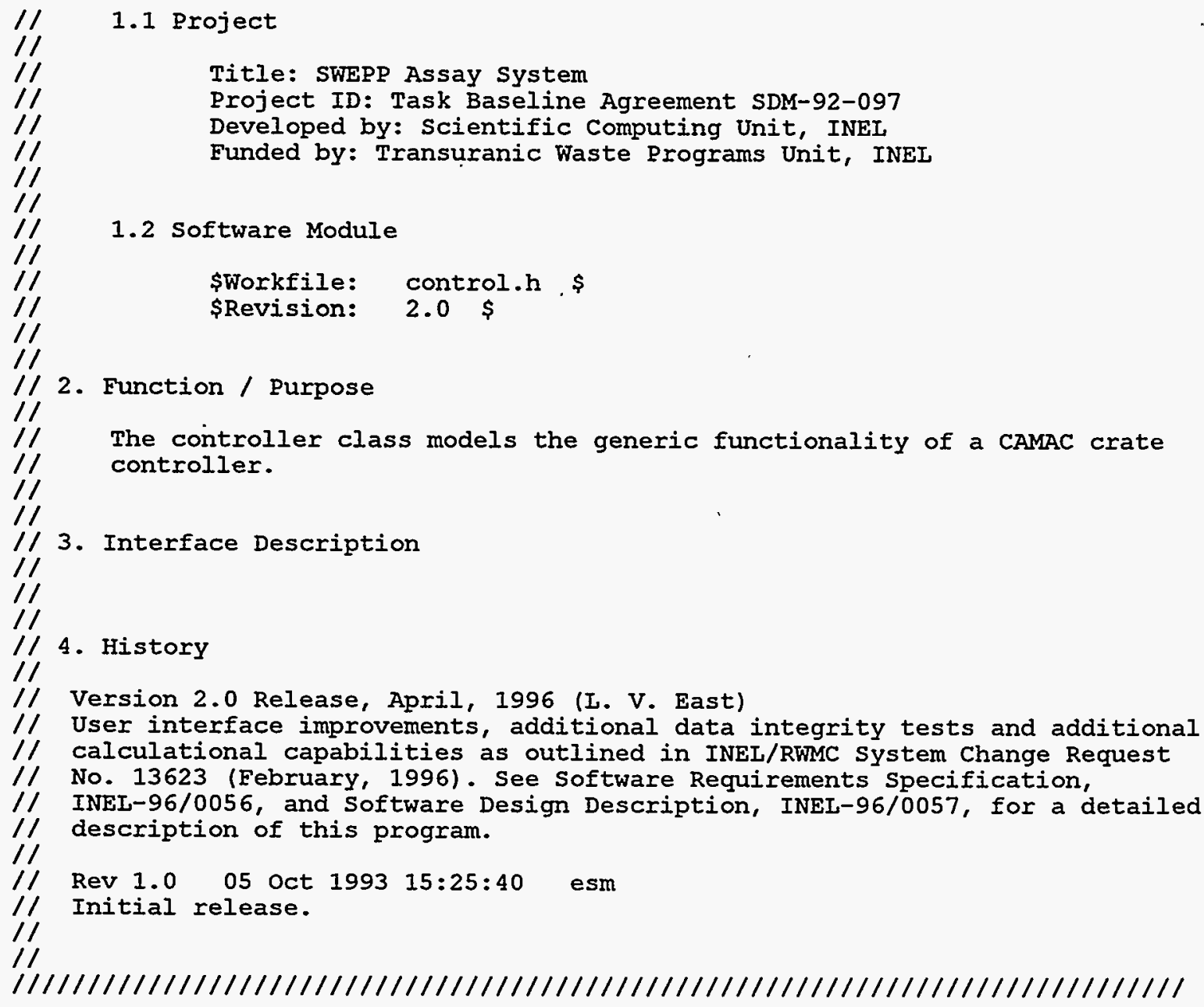

// 4. History 


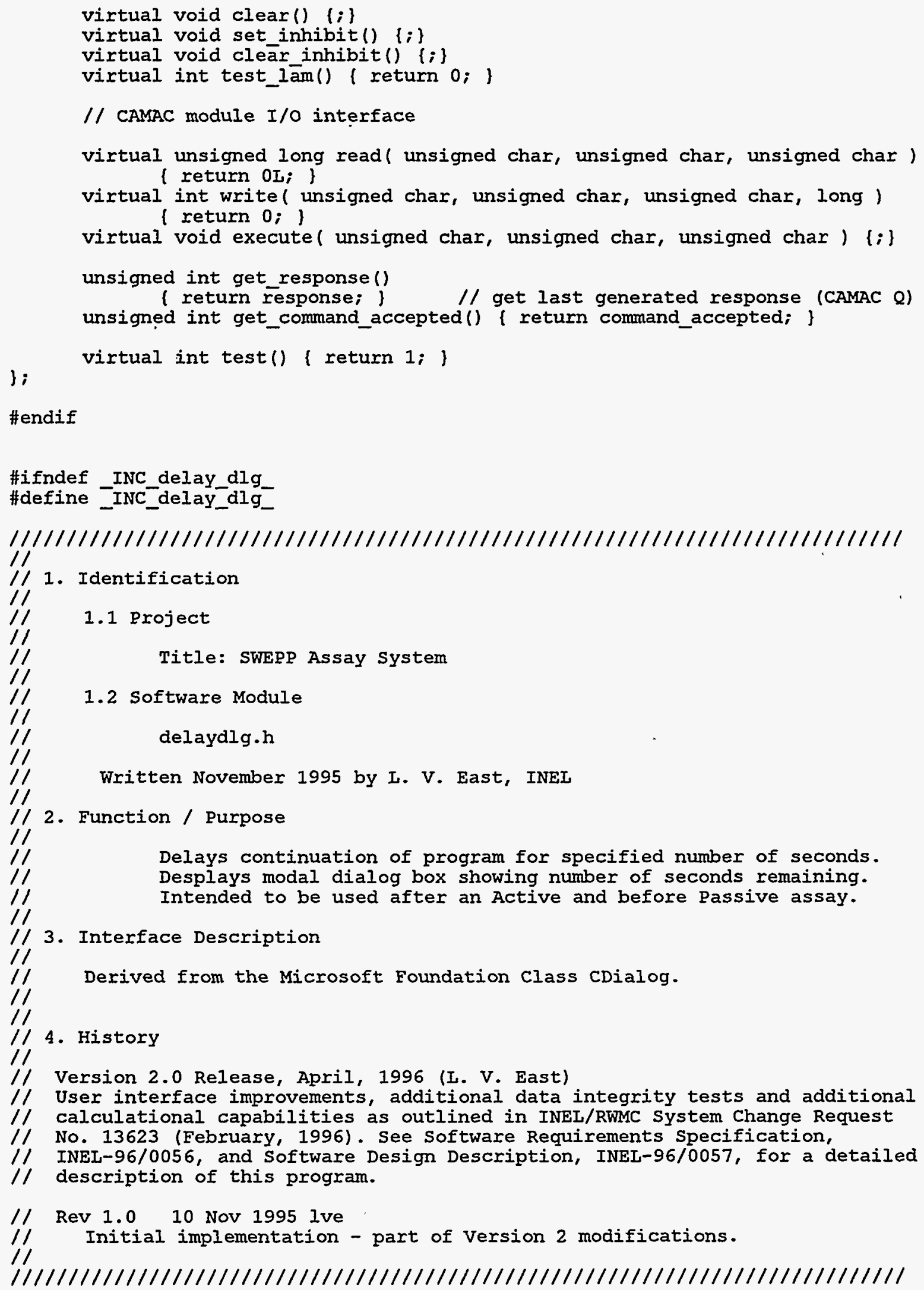

// 2. Function / Purpose 
INEL-96/0057

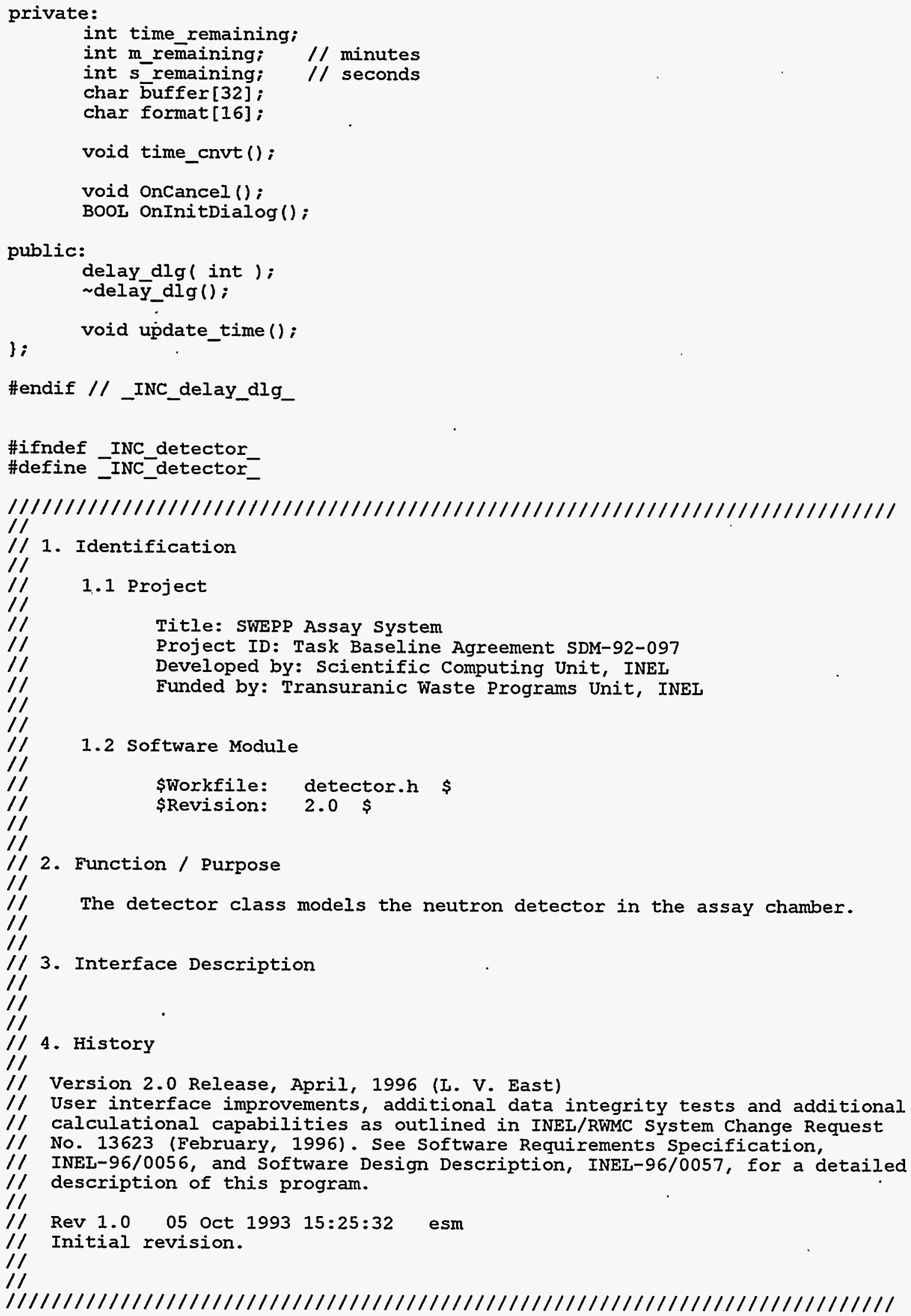

$1 /$

$1 /$

$1 /$

// 2. Function / Purpose 


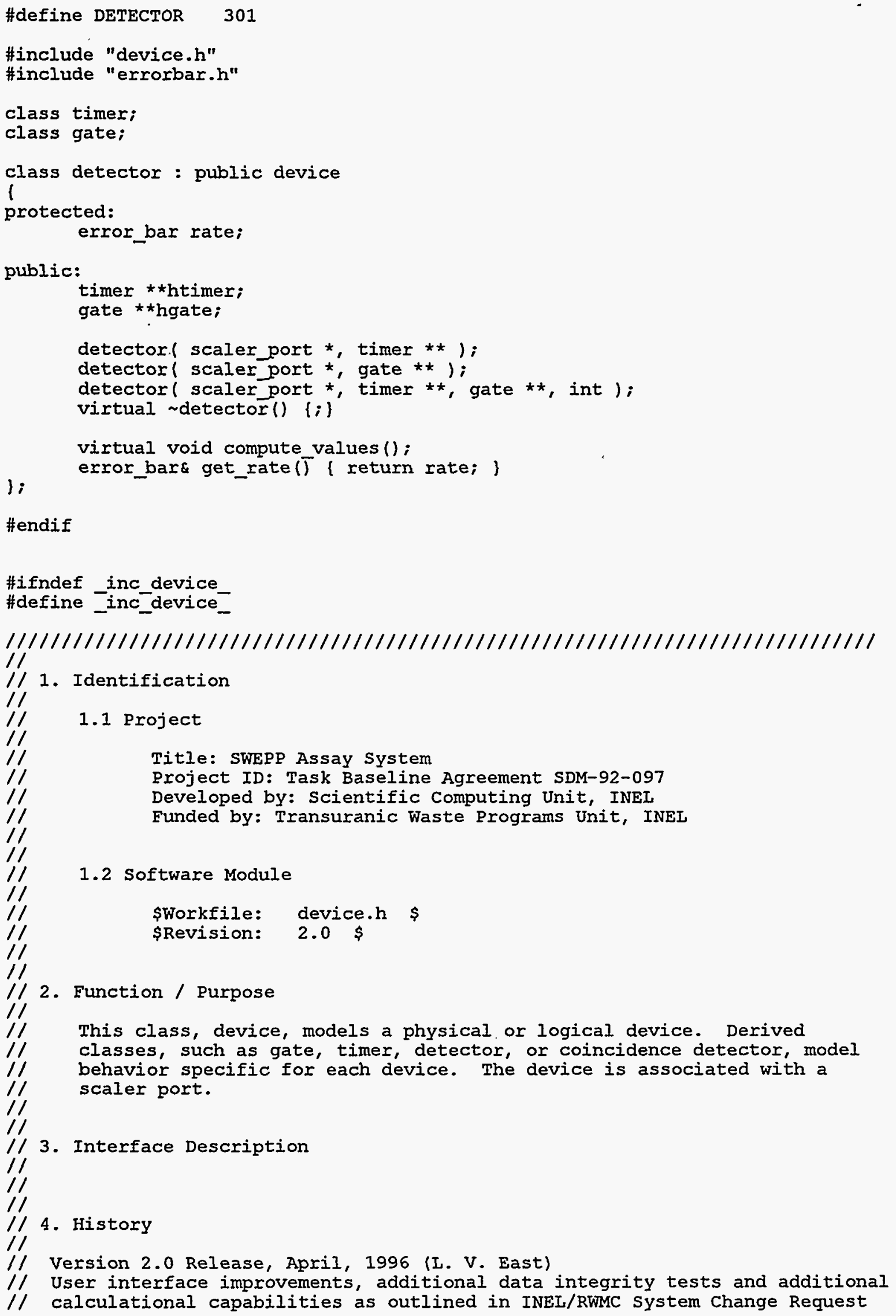

\#endif

\#ifndef inc_device \#define -inc_device-

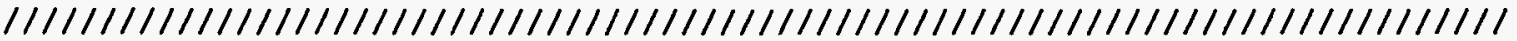

$1 /$

/1/

// 2. Function / Purpose

$1 /$

// This class, device, models a physical or logical device. Derived

// classes, such as gate, timer, detector, or coincidence detector, model

// behavior specific for each device. The device is associated with a

// scaler port.

$1 /$

$1 /$

// 3. Interface Description

$1 /$

$1 /$

$1 /$

// 4. History

$1 /$

// Version 2.0 Release, April, 1996 (L. V. East)

// User interface improvements, additional data integrity tests and additional

// calculational capabilities as outlined in INEL/RWMC system Change Request 


\section{INEL-96/0057}

// No. 13623 (February, 1996). See Software Requirements specification,

// INEL-96/0056, and Software Design Description, INEL-96/0057, for a detailed

$/ /$ description of this program.

$1 /$

// Rev 1.0 05 Oct 1993 15:25:26 esm

//Initial revision.

$1 /$

11

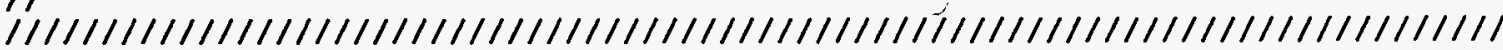

class scaler_port;

class device

I

char *name;

int type;

protected:

scaler_port *pscaler_port;

public:

device ( scaler_port *, int );

device ( scaler port *);

virtual device ();

void set name ( char *);

char *get name() ( return name; )

int is_type( int type) ( return this->type $==$ type; \}

unsigned long get_count ():

\}

virtual void compüte_values() $(i)$

\#endif

\#ifndef INC_device_list

\#define INC_device_list_

$/ / 1 / / 1 / 1 / 1 / 1 / 1 / 1 / 1 / 1 / 1 / 1 / 1 / 1 / 1 / 1 / 1 / 1 / 1 / 1 / 1 / 1 / 1 / 1 / 1 / 1 / 1 / 1 / 1 / 1 / 1 / 1 / 1 / 1 / 1 / 1$

$1 /$

// 1. Identification

$1 /$

$1 /$

$1 /$

// 3. Interface Description

1.1 Project

Title: SWEPP Assay system

Project ID: Task Baseline Agreement SDM-92-097

Developed by: Scientific Computing Unit, INEL

Funded by: Transuranic Waste Programs Unit, INEL

\subsection{Software Module}

\$Workfile: devlist.h \$

\$Revision: 2.0 \$

2. Function / Purpose

The device list models the list of devices associated with a scaler port. In the usual case there is only a single device.

11 
$1 /$ 4. History

// Version 2.0 Release, April, 1996 (I. V. East)

// User interface improvements, additional data integrity tests and additional

II calculational capabilities as outlined in INEL/RWMC system Change Request

$/ /$ No. 13623 (February, 1996). See Software Requirements specification,

// INEL-96/0056, and Software Design Description, INEL-96/0057, for a detailed $/ /$ description of this program.

$/ / /$ Rev $1.0 \quad 05$ Oct $1993 \quad 15: 25: 40$ esm

// Initial release.

$1 /$

$1 /$

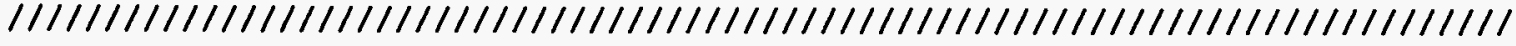

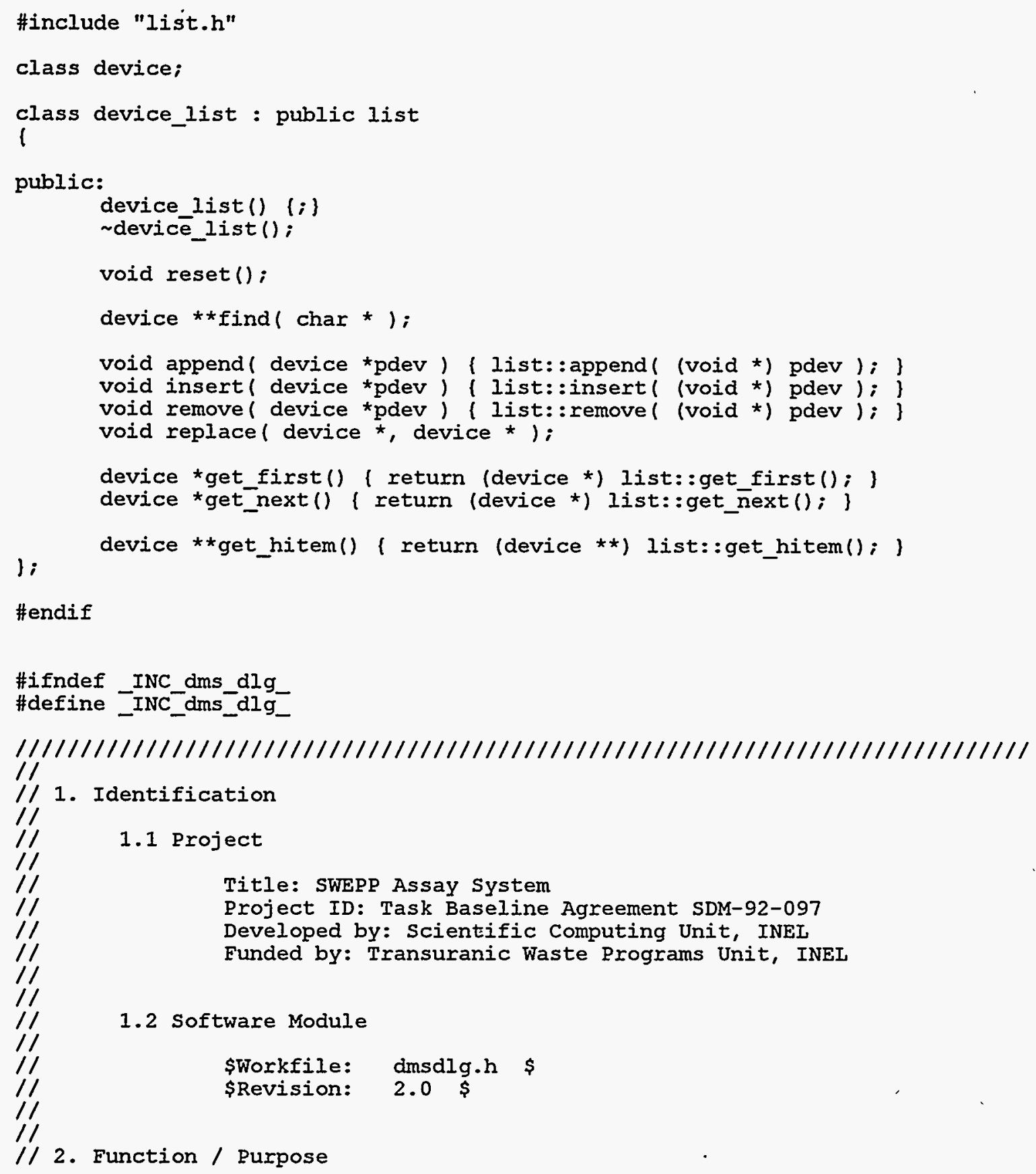

// 2. Function / Purpose 


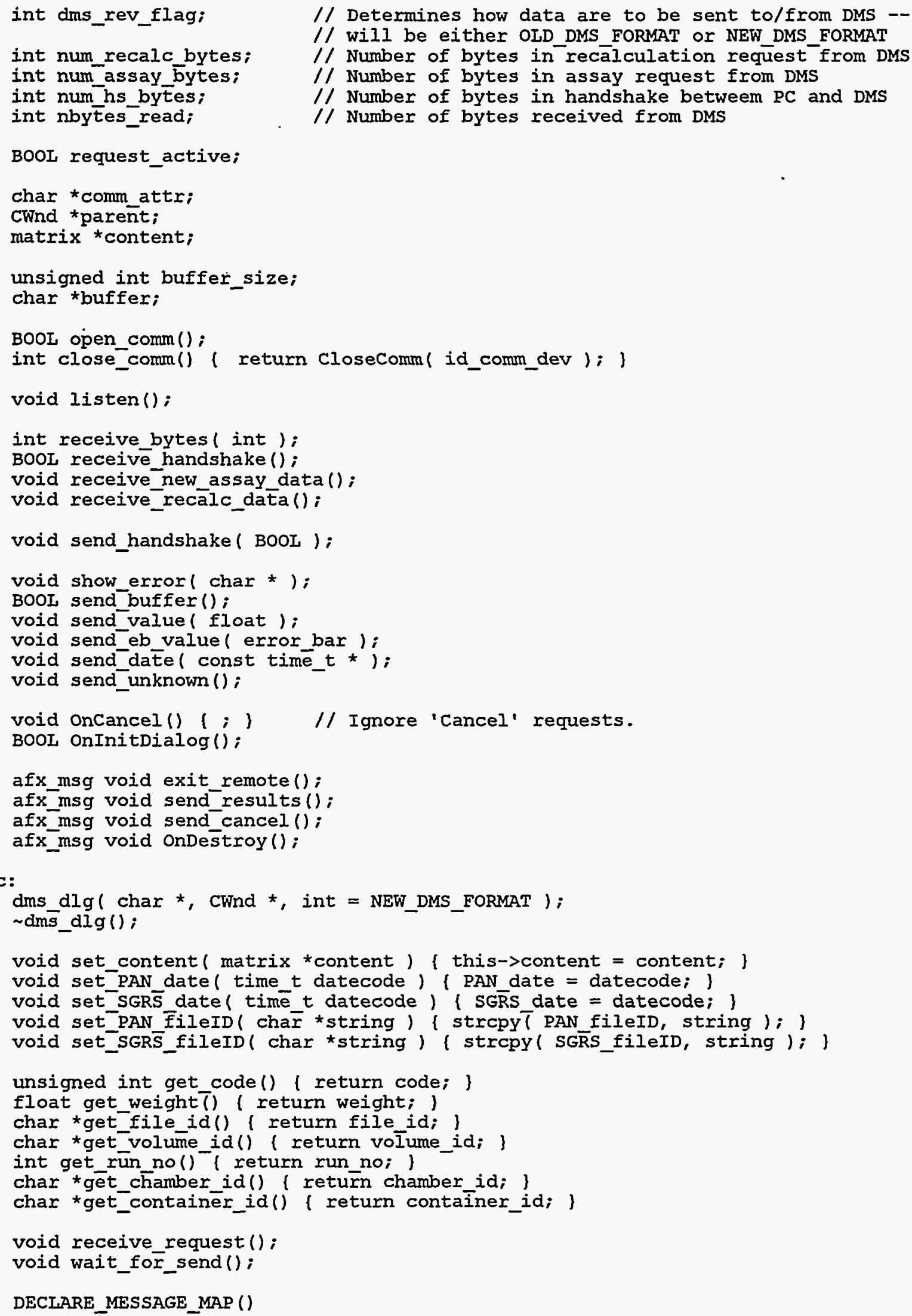




\section{INEL-96/0057}

\#endif

$$
\begin{aligned}
& \text { \#ifndef INC_error_bar_ } \\
& \text { \#define INC_error_bar_ }
\end{aligned}
$$

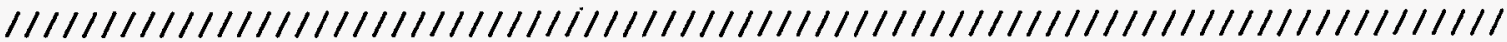

$1 /$

// 1. Identification

11

$1 /$

// 3. Interface Description

\subsection{Project}

Title: SwEPP Assay system

Project ID: Task Baseline Agreement SDM-92-097

Developed by: Scientific Computing Unit, INEL

Funded by: Transuranic Waste Programs Unit, INEL

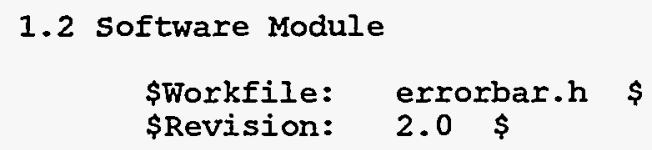

2. Function / Purpose

The error bar class models an error bar, that is a value and its one standard deviation error.

4. History

Version 2.0 Release, April, 1996 (I. V. East)

User interface improvements, additional data integrity tests and additional calculational capabilities as outlined in INEL/RWMC system Change Request No. 13623 (February, 1996). See Software Requirements Specification, INEL-96/0056, and Software Design Description, INEL-96/0057, for a detailed description of this program.

Rev 1.0 05 Oct $1993 \quad 15: 25: 28$ esm

Initial release.

5. References

5.1 References regarding error propagation and manipulation:

5.1 .1 G. F. Knoll, "Radiation Detection and Measurement," John Wiley and Sons, New York, NY, 1979, pp 131-135.

5.1.2 P. R. Bevington, "Data Reduction and Error Analysis for the Physical Sciences," McGraw Hill, New York, 1969, Chapter 4.

5.1 .3 J. R. Taylor, "An Introduction to Error Analysis," oxford University Press, 1982, Part I.

5.1.4 H. W. Coleman and W. G. Steele, "Experimentation and Uncertainty for Engineers," John Wiley \& Sons, 1989, Section 3-1.

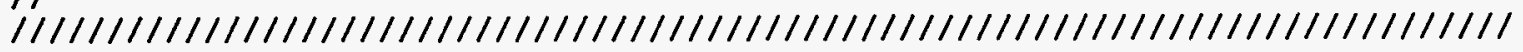

\#include <fstream.h>

\#include <iomanip.h> 


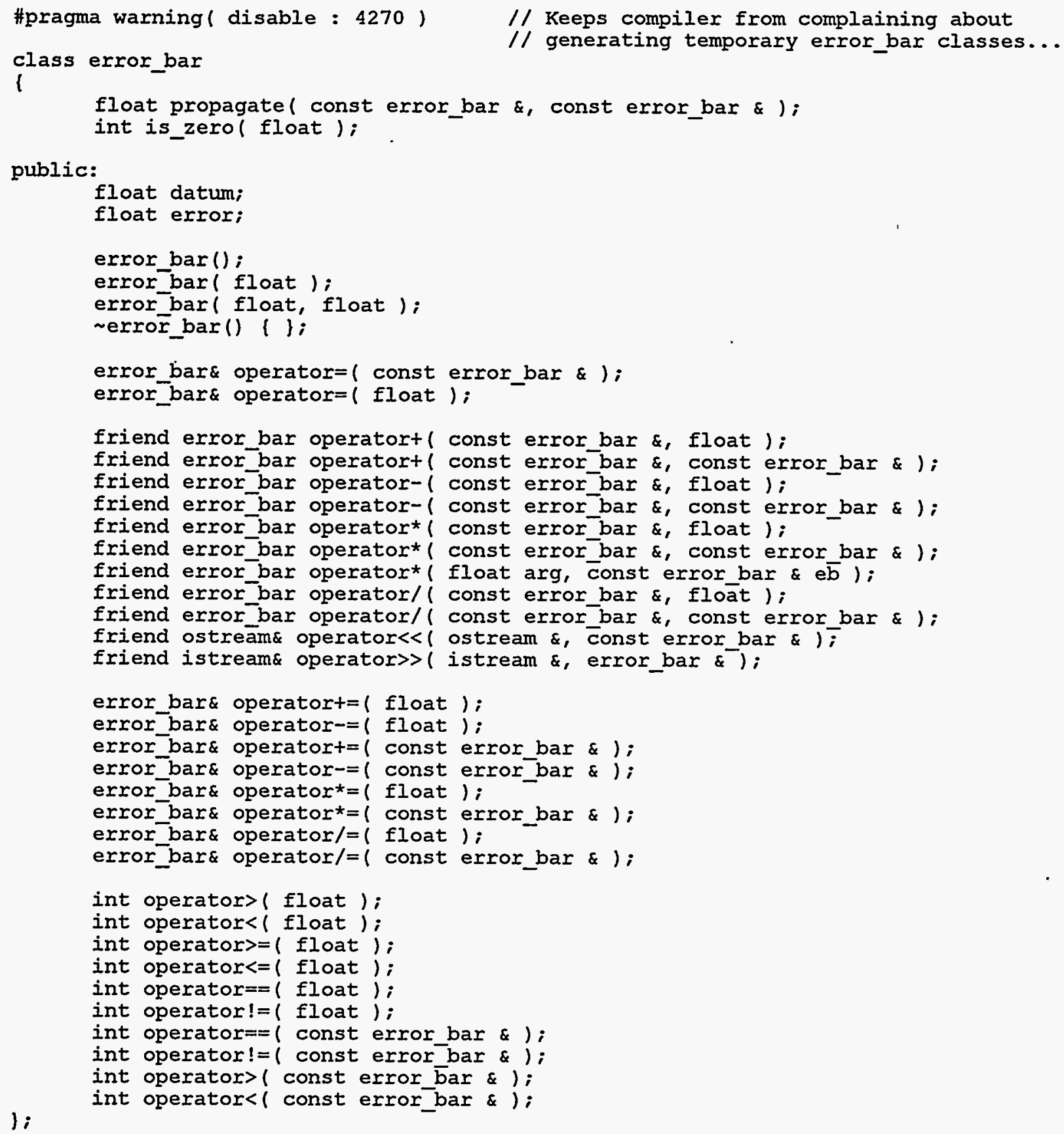

\#endif

\#ifndef INC file lim

\#define INC_file_lim_

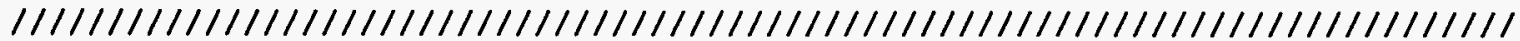

11

$/ / 1$. Identification

/1

11

II

11

1.1 Project

Title: SWEPP Assay system

Project ID: Task Baseline Agreement SDM-92-097

Developed by: Scientific Computing Unit,. INEL 


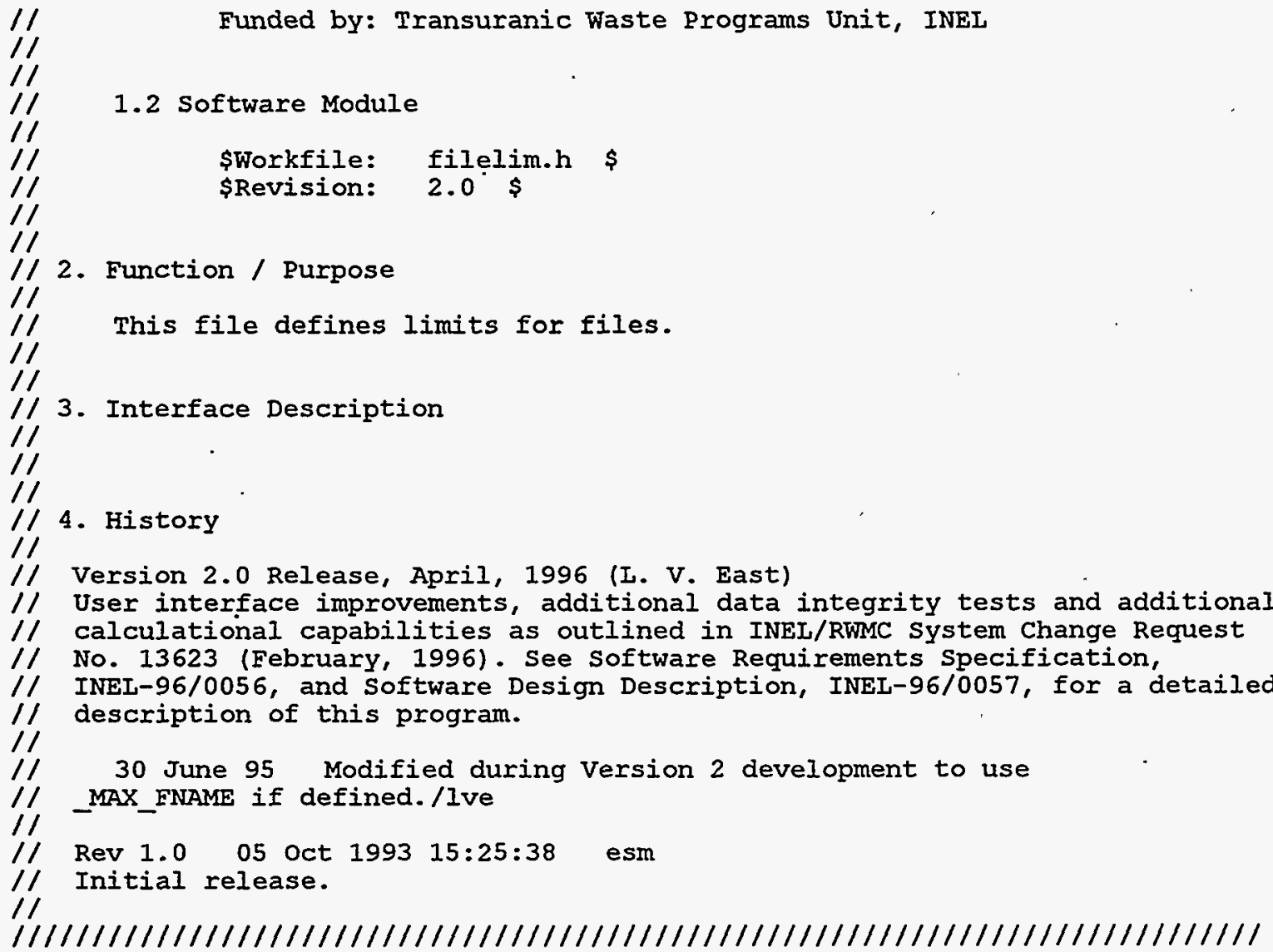

\#include <stdlib.h>

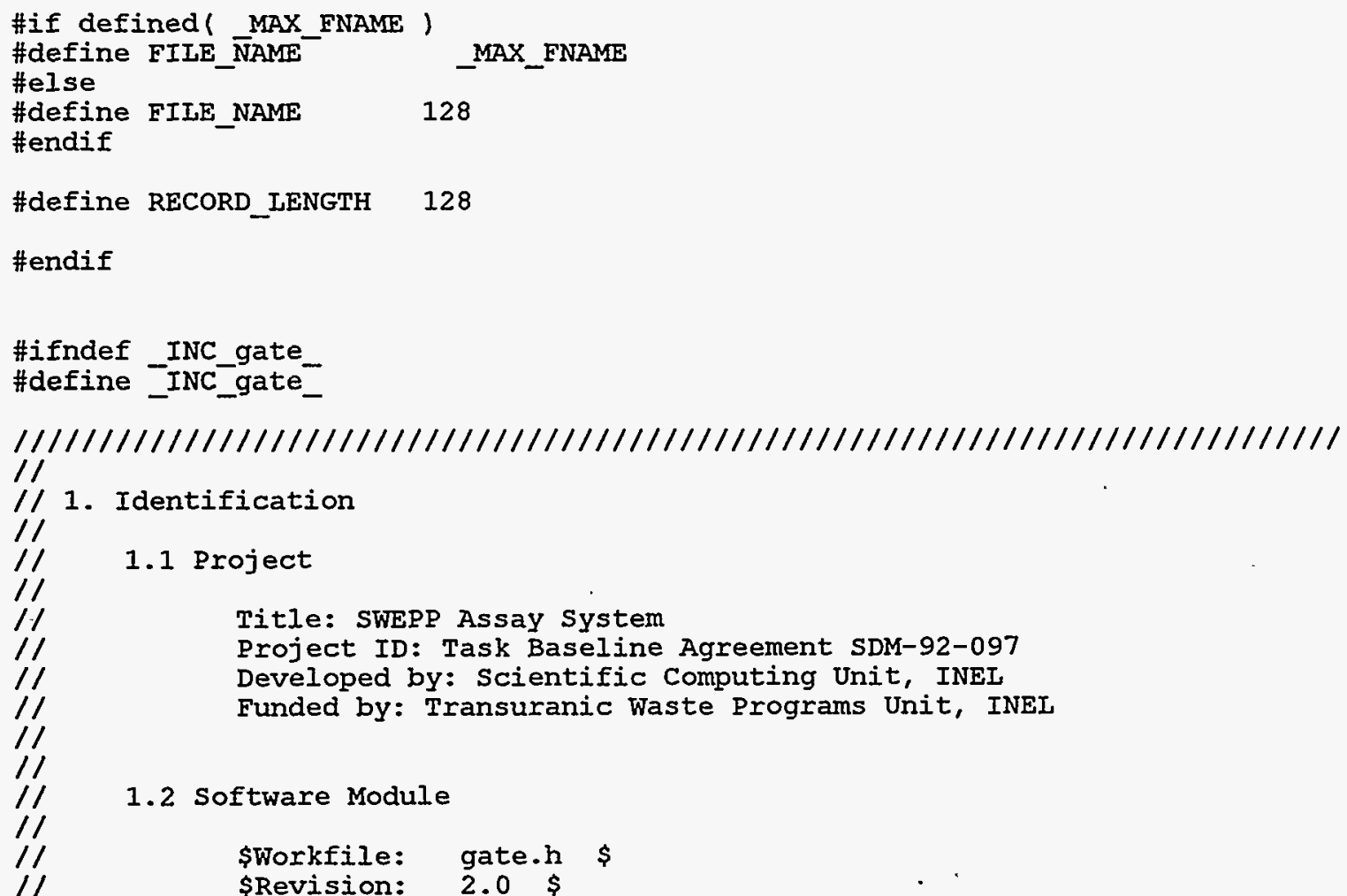

1.2 Software Module

$\begin{array}{ll}\text { \$Workfile: } & \text { gate.h } \\ \text { SRevision: } & 2.0 \$\end{array}$ 


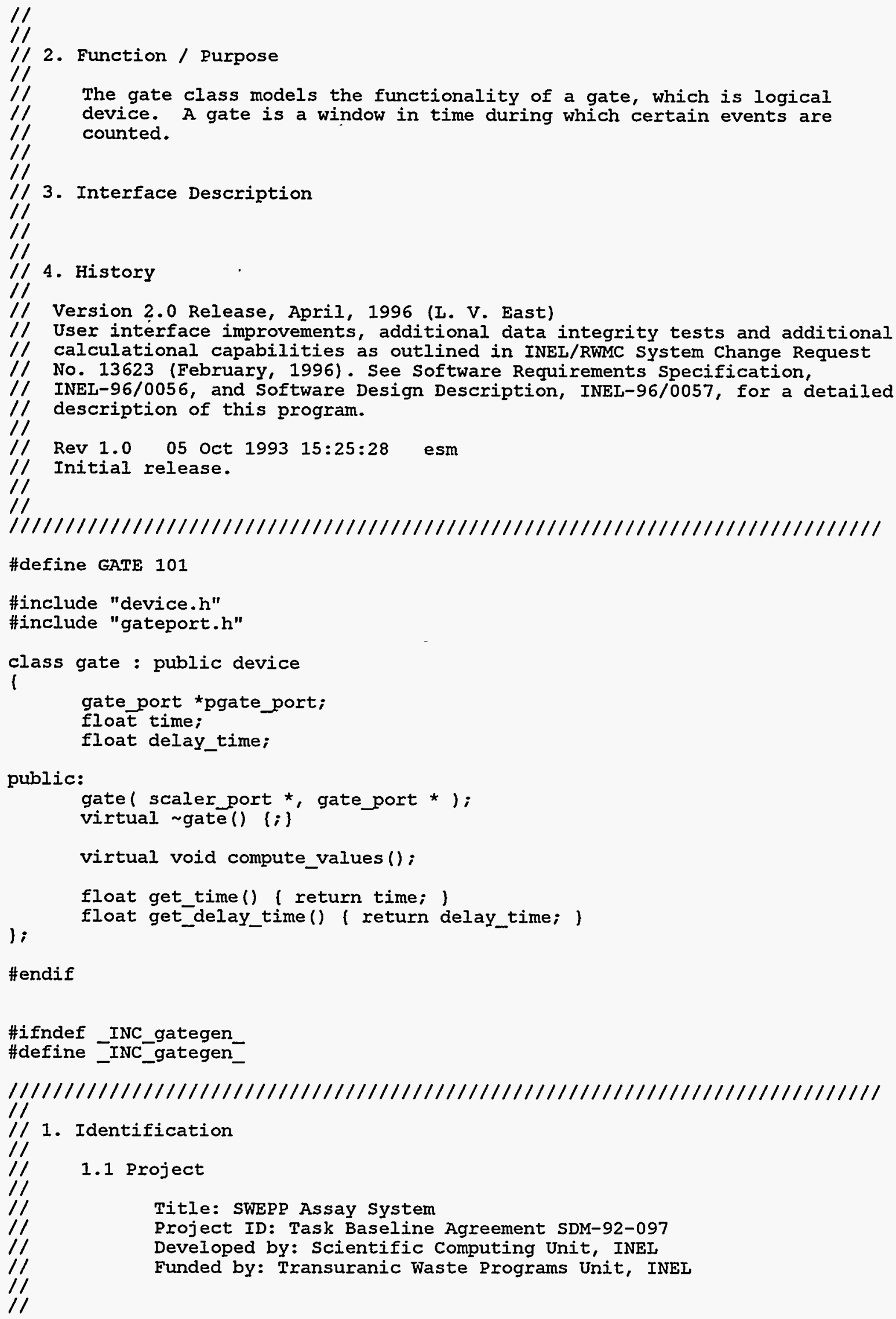




\section{INEL-96/0057}

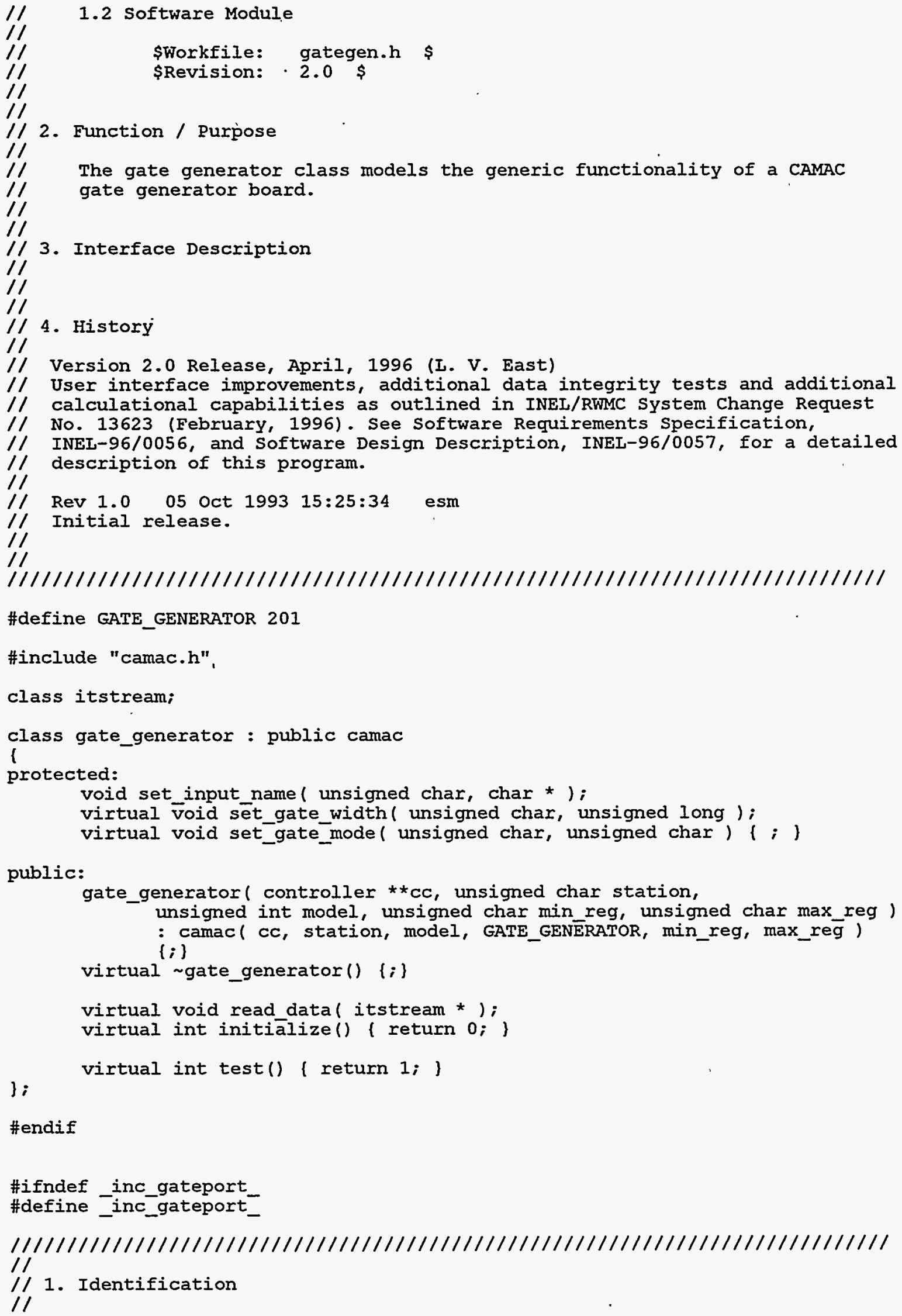




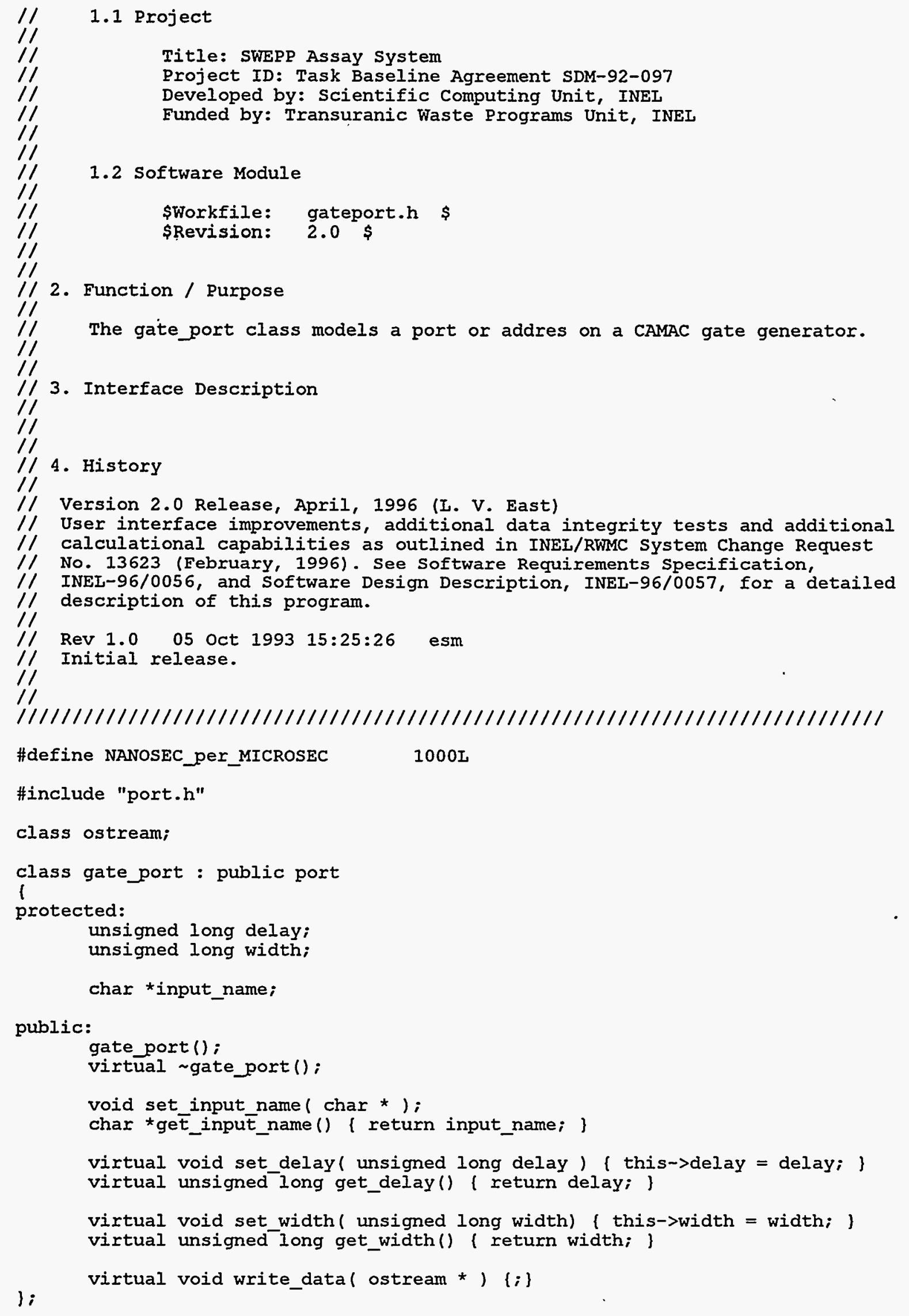




\section{INEL-96/0057}

\#endif

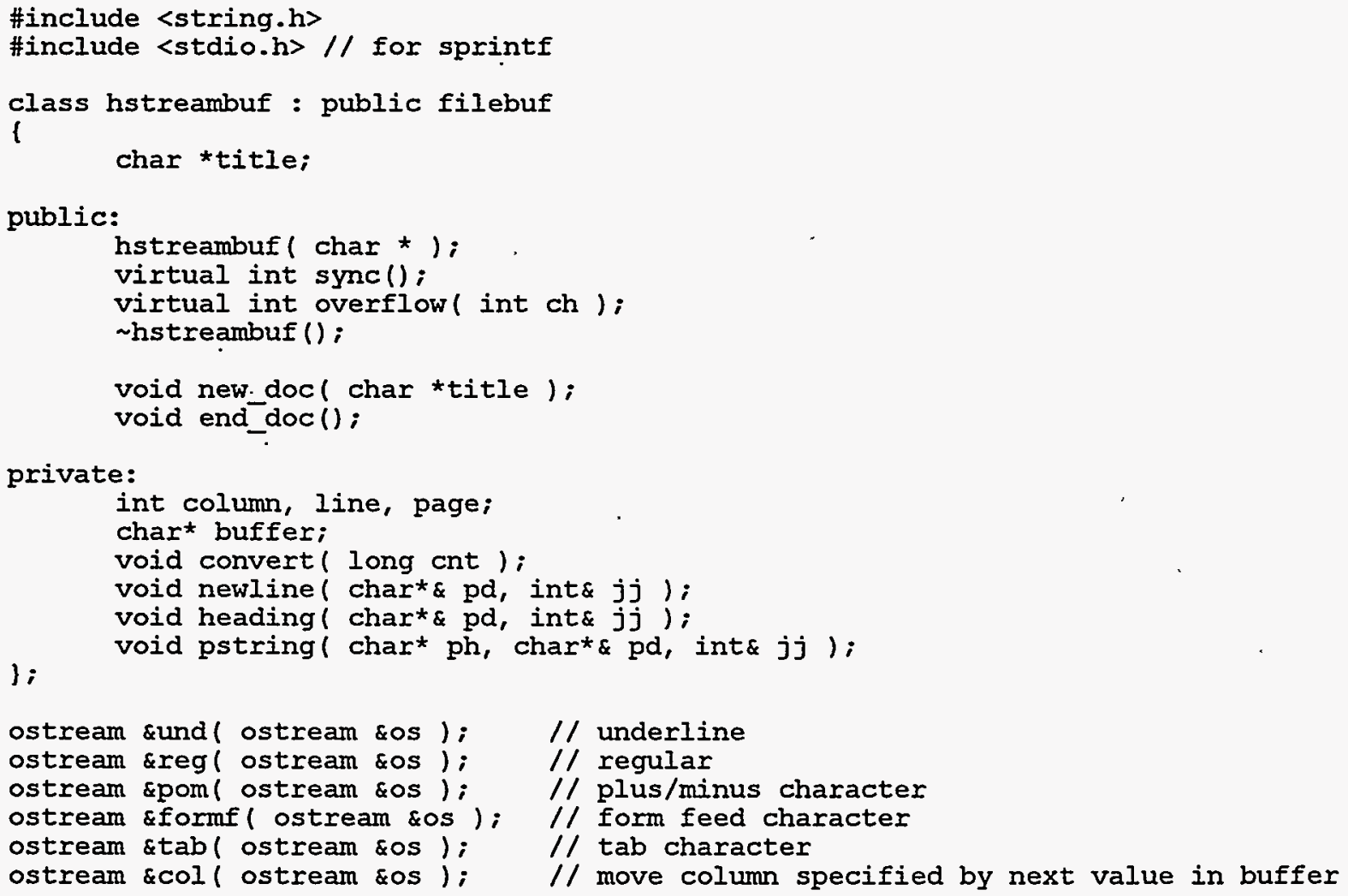

// 3. Interface Description

\subsection{Project}

Title: SWEPP Assay system

Project ID: Task Baseline Agreement SDM-92-097

Developed by: Scientific Computing Unit, INEL

Funded by: Transuranic Waste Programs Unit, INEL

\subsection{Software Module}

\$Workfile: itstream.h $\$$ \$Revision: 2.0 \$

2. Function / Purpose

The itstream class models an input token stream. 


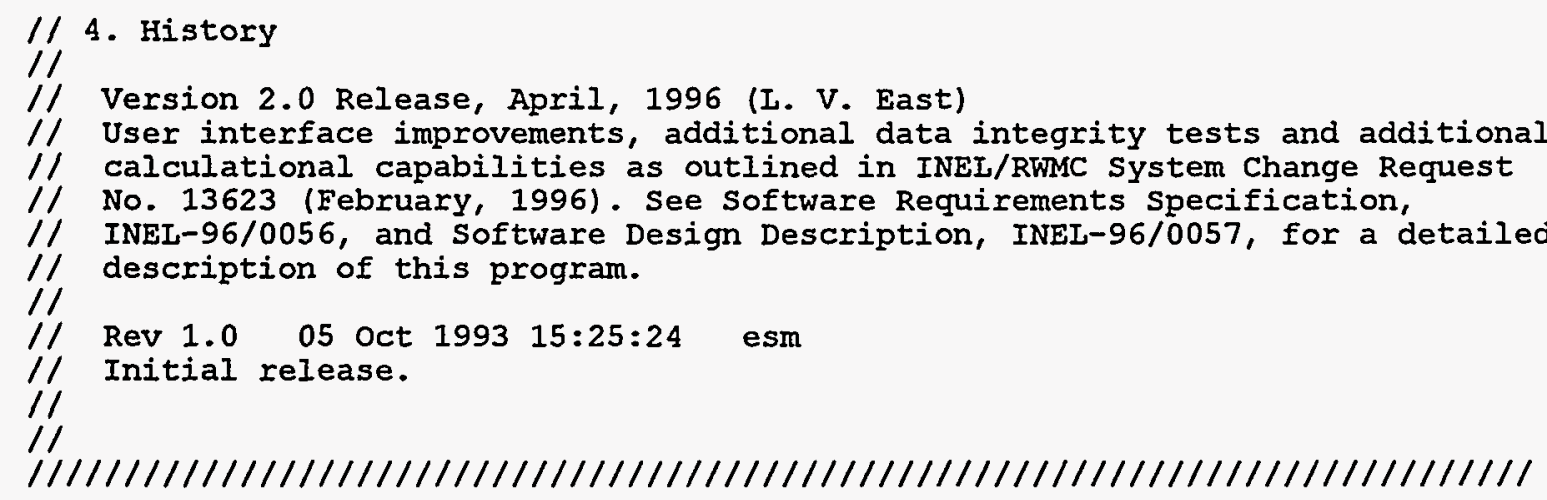

\section{\#include <fstream.h> \\ \#include "errorbar.h" \\ \#include "filelim.h"}

\#define No_BUFFER 1

class itstream : public ifstream

1

char buffer[RECORD_LENGTH];

int section;

streampos last pos;

public:

itstream ( $\operatorname{char} *$, int $=0$ );

void rewind ( const char delim[] = " $\backslash t "$ );

char* get token ( const char delim[] = " $(t ")$;

int find ( const char *, ios: :seek dir dir = ios: :cur, const char delim[] = "Tt");

int find_section( const char *, ios: :seek_dir dir = ios: :cur,

void end section(); const char delim[] = " $\mid t "$ )

int get val ( const char *, unsigned char \&);

void get val ( unsigned char \& );

int get $\bar{v}$ al ( const char $\star$, int \&);

void get val ( int \& );

int get val( const char *, unsigned int \&);

void get_val ( unsigned int \& ):

int get $\bar{v}$ al ( const char *, long \& );

void ge $\bar{t}$ val (long \&);

int get $\bar{v} a l$ ( const char *, unsigned long \&);

void get_val ( unsigned long \& );

int get val ( const char *, float \&);

void ge $\bar{t}$ _val ( float \& );

int get val ( const char *, error bar \&);

void get_val ( error_bar \& );

char *get_text ( const char delim[] = "\t");

);

\#endif

\#ifndef INC link

\#define INC_link_

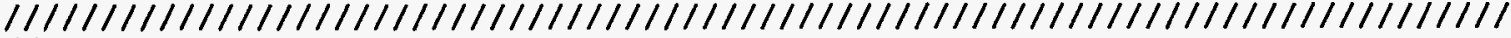

11

// 1. Identification

$1 /$

1.1 Project 
1. Identification

\subsection{Project}

Title: SWEPP Assay System

Project ID: Task Baseline Agreement SDM-92-097

Developed by: Scientific Computing Unit, INEL

Funded by: Transuranic Waste Programs Unit, INEL

\subsection{Software Module}

\$Workfile: list.h \$

\$Revision: 2.0 \$

11

$1 /$

// 2. Function / Purpose

Uersion 2.0 Release, April, 1996 (L. V. East)

User interface improvements, additional data integrity tests and additional

// calculational capabilities as outlined in INEL/RWMC system Change Request

// No. 13623 (February, 199.6). See Software Requirements Specification,

// INEL-96/0056, and Software Design Description, INEL-96/0057, for a detailed

// description of this program.

/I

// Rev $1.0 \quad 05$ Oct $1993 \quad 15: 25: 24$ esm

// Initial release.

$1 /$

$1 /$

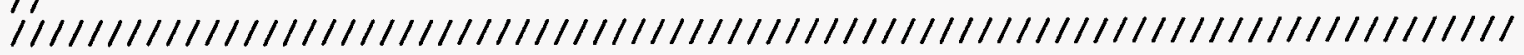

\#include "filelim.h" // for FILE_NAME (max length of a file name).

class link;

class list

i

link *first;

link *Iast;

link * current;

int count;

void initialize();

protected:

char file name [ FILE NAME ];

int has_write_permiss̄ion: 


\section{INEL-96/0057}

list ();

list ( char *);

rist ():

void reset();

void insert ( void *);

void append ( void *);

void remove ( void $*$ );

void *replace ( void *);

void *get_first ():

void * get_next ();

void * get_current ();

void *get_last ();

void $* \dot{*}$ get_hitem();

public:

int can_write() \& return has_write_permission; )

virtual int read data() ( return $0 ;$ \}

virtual void write_data() $\{$;

);

int get_count() ( return count; \}

\#endif

\#ifndef _INC_login_dlg_

\#define _INC_login_dlg_

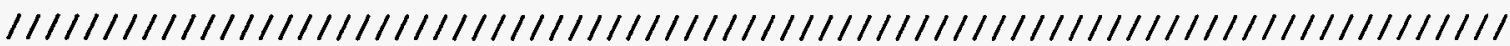

$1 /$

// 1. Identification

/1

II

/1

11

11

11

$1 /$

11

11

/1 2

$1 /$

// 3. Interface Description

// 4. History

$1 /$

// Version 2.0 Release, April, 1996 (I. V. East)

// User interface improvements, additional data integrity tests and additional

// calculational capabilities as outlined in INEL/RWMC System Change Request

// No. 13623 (February, 1996). See Software Requirements Specification,

// INEL-96/0056, and Software Design Description, INEL-96/0057, for a detailed

// description of this program. 


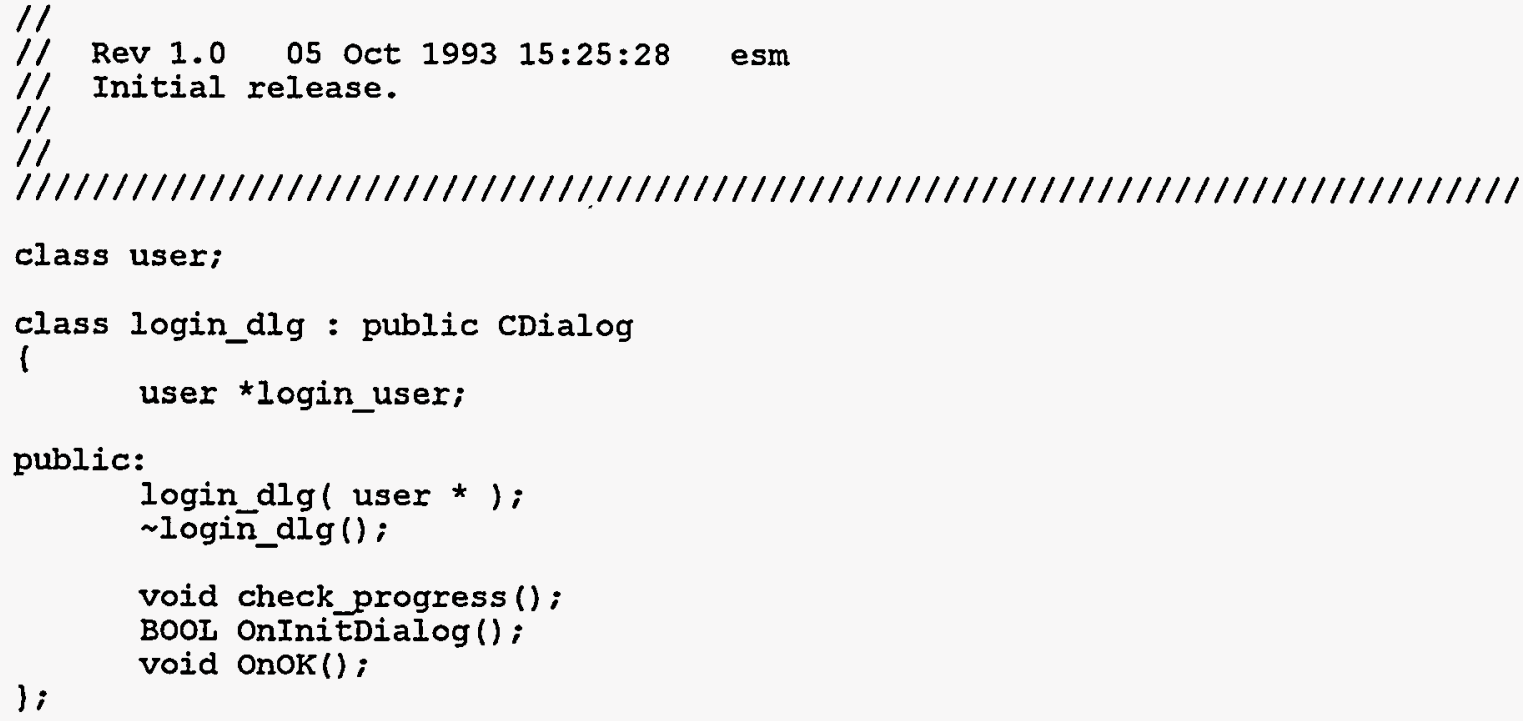

//2. Function / Purpose 
$1 /$

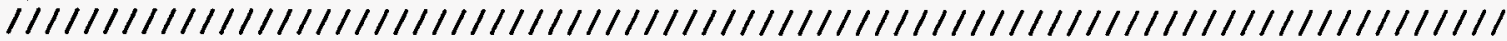

\#include <afxext.h>

// For status bar object definitions.

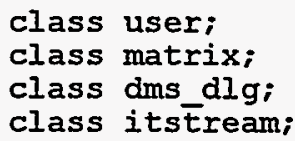

public:

main_wnd () ; 


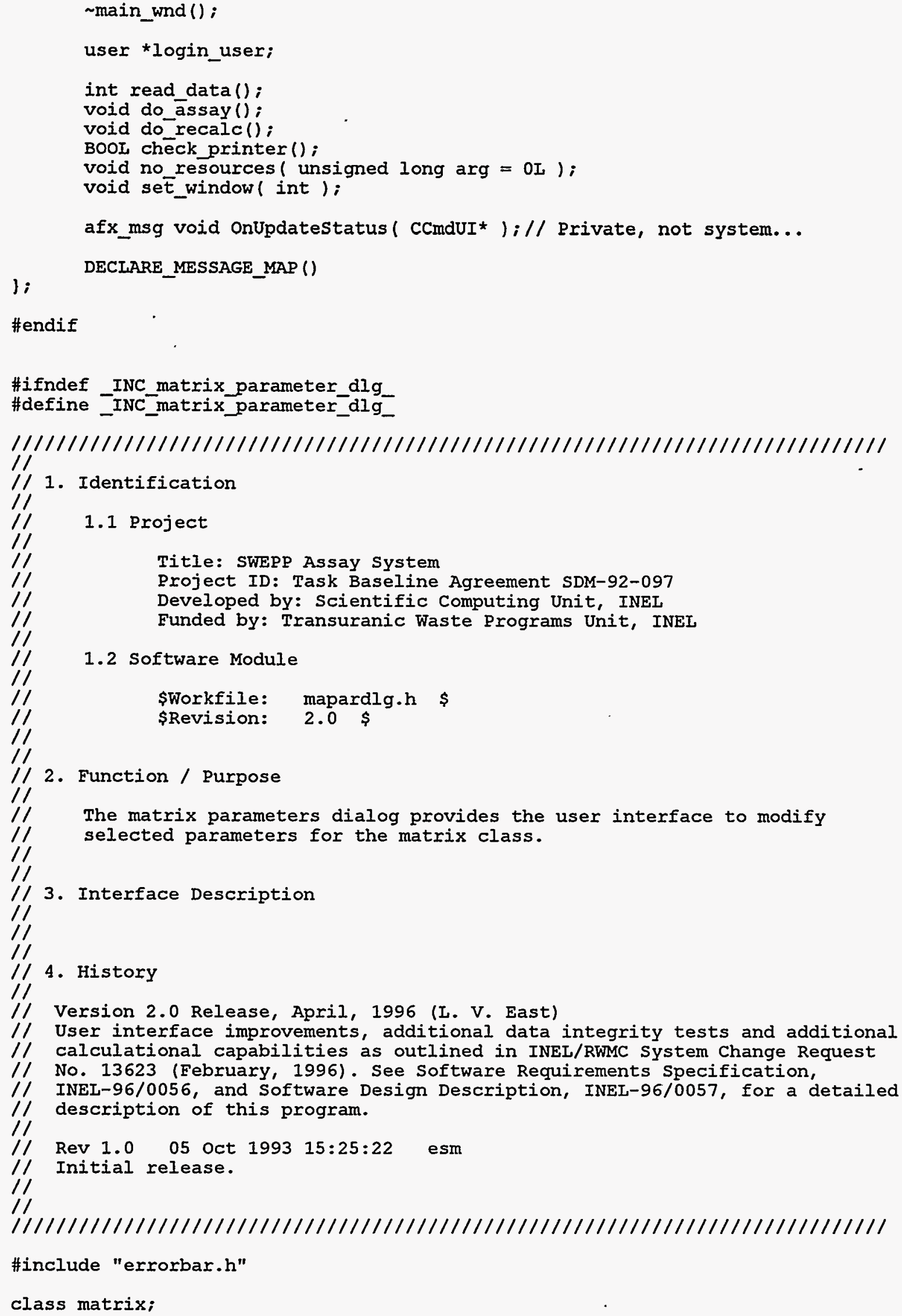

// 2. Function / purpose

// The matrix parameters dialog provides the user interface to modify 
INEL-96/0057

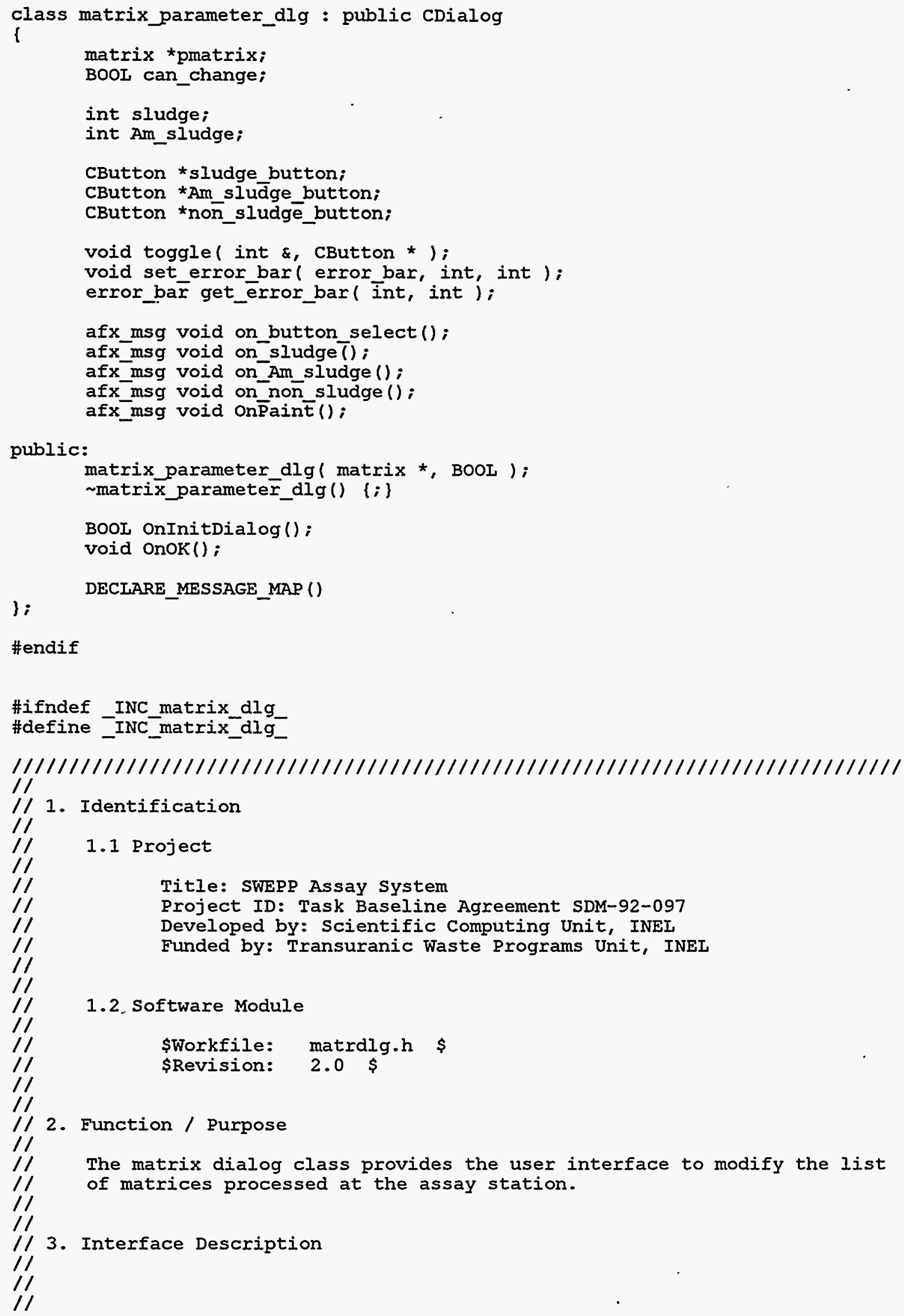

11

// 2. Function / Purpose

11

// The matrix dialog class provides the user interface to modify the list

// of matrices processed at the assay station.

$1 /$

$1 /$

// 3. Interface Description

Title: SWEPP Assay System

Project ID: Task Baseline Agreement SDM-92-097

Developed by: Scientific Computing Unit, INEI

Funded by: Transuranic Waste Programs Unit, INEL

1.2. Software Module

\$Workfile: matrdlg.h \$

\$Revision: 2.0 \$

/1 
// 4. History

11

// Version 2.0 Release, April, 1996 (L. V. East)

// User interface improvements, additional data integrity tests and additional

// calculational capabilities as outlined in INEL/RWMC system Change Request

// No. 13623 (February, 1996). See Software Requirements Specification,

// INEL-96/0056, and Software Design Description, INEL-96/0057, for a detailed

// description of this program.

// Rev 1.0 05 Oct 1993 15:25:26 esm

// Initial release.

$1 /$

11

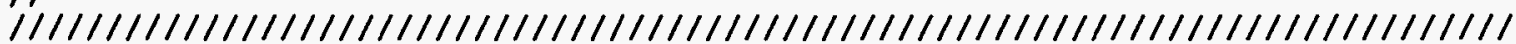
class matrix;

class main wnd;

class matrix_dlg : public cDialog

l

char *title;

main wnd * parent;

char *text;

unsigned int content_code;

matrix *pmatrix;

int sel;

BOOL changed; $\quad / /$ Flag to indicate whether list has been changed

BOOL can_write; // TRUE if underlying matrix file is write enabled.

CListBox *list_box;

Bool get_dlg_data( int );

void set dlg-data();

BOoI is duplicate ();

void resset focus ( int):

int add_string():

void seE button state():

void set_matrix_parameters ();

afx_msg void on_add();

afx msg void on change ():

afx msg void on delete ();

afx msg void on selchange ();

afx_msg void on $\bar{P}$ aint ();

public:

matrix_dlg ( main_wnd * );

matrix dlg();

BOOL OnInitDialog () :

void OnOK();

void oncancel ();

li

DECLARE_MESSAGE_MAP ()

\#endif

\#ifndef INC matrix

\#define _INC_matrix_-

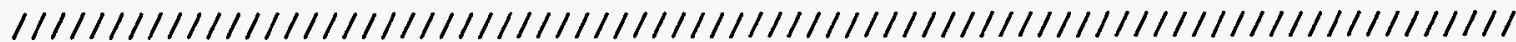

$1 /$

// 1. Identification 


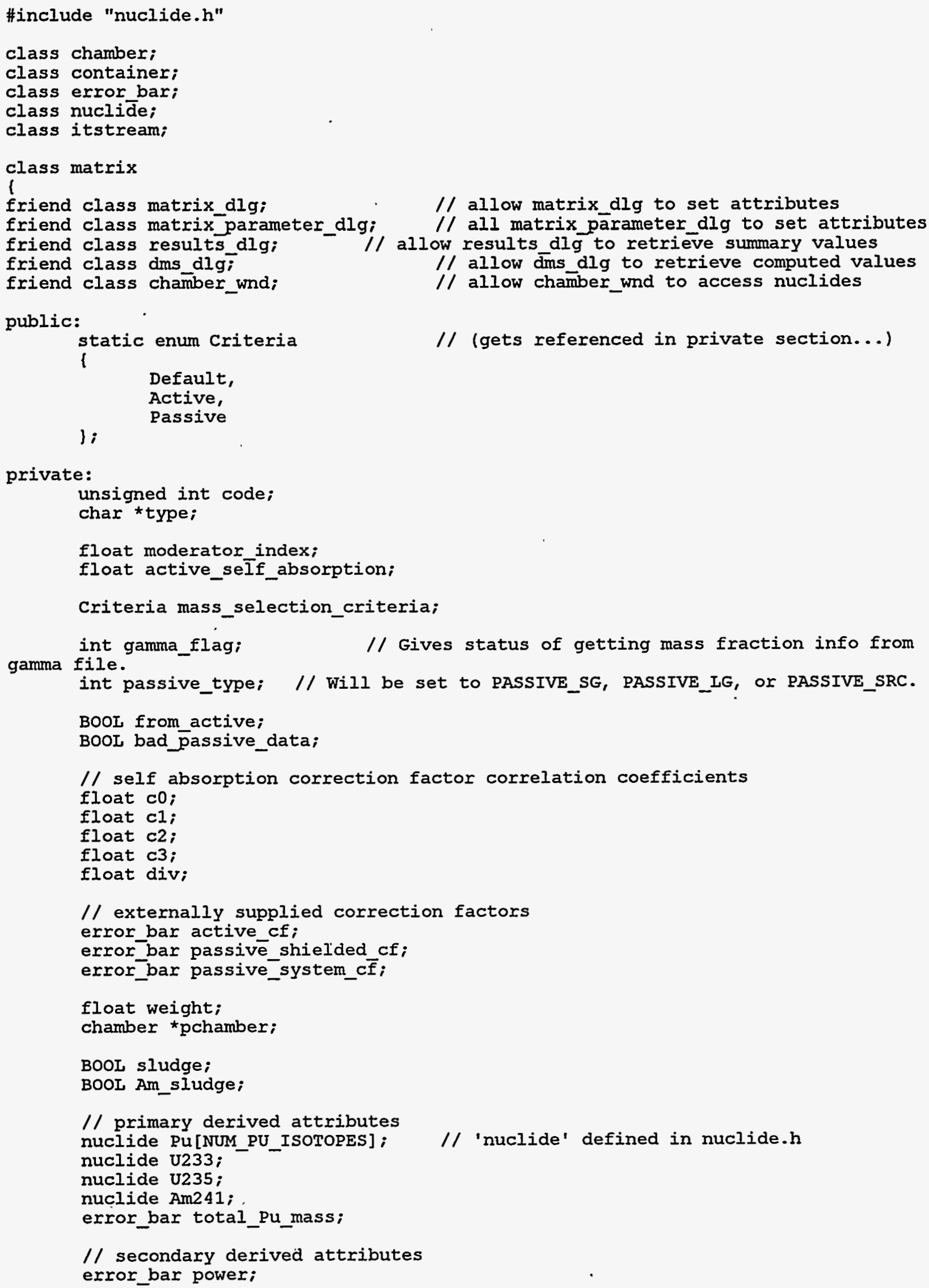


INEL-96/0057

error_bar power_density;

error_bar alpha_activity;

error_bar $\mathrm{Pu} e q u \overline{i v}$ activity;

error bar total activity:

error_bar TRU_activity;

error bar TRU activity_concentration;

error_bar fissile_gram_equivalent;

// interally.used, derived attributes

float absorber index;

error bar active Pu mass;

error_bar passive $\mathrm{Pu}$ mass;

errox_bar Am241_NE_mass; // Am-241 mass derived from "neutron excess"

error_bar U_cf;

error bar active shielded total rate;

error bar active_flux monitor_rate;

error_bar active_barrēl_flux_monitor_rate;

error bar passive shielded total rate;

error bar passive system tötal rāte;

error bar passive shieldē coiñcidence rate;

error_bar passive_system_cōincidence_rāte;

BOoI rate_limited;

// constants for absorber index equation

float AI DEFAULT;

float AIMMIN;

// constants for moderator index equation

float $\mathrm{A} 0, \mathrm{~A} 1, \mathrm{~A} 2, \mathrm{~A} 3$;

float MI MIN;

float MI_MAX;

// active matrix correction factor parameters

float AI THRESHOLD;

float AMC $\bar{C}$ AI_CO;

float $\mathrm{AMCE}$ AI C1;

float MI THRESHOLD;

float $A M \bar{C} F$ MI $\mathrm{CO}$;

float $\mathrm{AMCF} M \mathrm{MI}^{-} \mathrm{C}$;

// mass calculation coefficients for active calculation

float AI MI CO;

float $\mathrm{AI} M \mathrm{MI}^{-} \mathrm{Cl}$;

float ACTIVE BACKGROUND SCALE FACTOR;

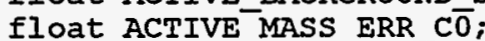

float ACTIVE MASS ERR C1;

float ACTIVE_MASS_ERR_C2;

// constants for passive mass calculations

float SYSTEM_COINCIDENCE_CF_CO;

float SYSTEM COINCIDENCE ${ }^{-}{ }^{-} \mathrm{Cl}$;

float SHIELDĒD COINCIDENCE_CF_CO;

float SHIELDED COINCIDENCE_CF_C1;

// final mass calc paramters

float MASS_THRESHOLD;

// americium sludge mass equation coefficients

float AM SLUDGE MASS C0;

float AM SIUDGE MASSCI;

float AM SIUDGE_MASS_C2;

float AM-SLUDGE MASS C 3 ; 
$/ /$ internal methods

void initialize();

BOOL flux_rates_ok()

$/ / \star \star \star$ Added in Version $2 * \star \star$

I

return (active barrel flux monitor rate $>0.0 f \& \&$

) active_fiux_monitor_rate $>10.0 f$ );

void calc_absorber_index();

void calc moderator index();

float calc_active_matrix_cf();

error_bar calc_active_Pu_mass (); / calc active mass

error_bar calc passive $\mathrm{pu}$ mass (); // calc passive mass

error_bar PAN_passive_pu_mass ( const char ${ }^{\star}$ ); // does the calculations

void Calc U mass (); - - // calc U-23x masses based on gamma data

error_bar calc_Am_mass ( BOOL = FAISE ); // calc Am-241 mass, not Am sludge error_bar calc_slüdge_Am mass (); // calc Am-241 mass if sludge is Am laced int Am_mass_from_ratios (T); // Am-241 mass from isotopic ratio data

float IANL_error_estimate( float matrix_cf, float active_mass ) // New in V2 $\mathfrak{l}$

\} return (float) fabs ( ACTIVE MASS ERR CO + ( ACTIVE MASS ERR CI + ACTIVE_MASS_ERR_C2 * matrix_Cf, * active_mass );

void calc power ();

void calc_activity () ;

void calc_FGE ();

void print_error_bar( ostream *, error_bar );

public:

matrix();

matrix( unsigned int, char *);

$\sim \operatorname{matrix}()$;

char Pu mass note [64];

// string describing how total Pu mass was derived. char Am_mass_note [64];

char Isōtopic̄note [64];

char U_corr_note_1[48];

char U_corr_note_2[48];

// string describing how Am-241 mass was derived.

// string describing how isotopic masses determined

// strings describing $U$ correction to active mass.

container *pcontainer;

void set_chamber ( chamber *pchamber ) ( this->pchamber = pchamber; \}

void set_gamma_flag ( int arg ) \{ gamma_flag = arg; \}

void set_weight' ( float weight) ( this->weight = weight; )

float get_weight() ( return weight; \}

BOOL is_rate_limited() ( return rate_limited; \}

BOOL is_activ̄e_ok();

BOOI is passive ok();

BOOL is_from_active() \{ return from_active; \}

BOOL is_from passive() (return !from_active; )

BOOI read data ( itstream *);

BOOL read_correlation_data ( itstream *);

BOOI read nuclide dată ( itstream *);

BOOL read Pu fractions ( itstream *):

void read_mass_ratios ( itstream * );

BOoI valid mass ( error bar \&value )

( return (value.datum != -1.0f \&\& value.error != -1.0f); \}

void write_data( ostream *); 


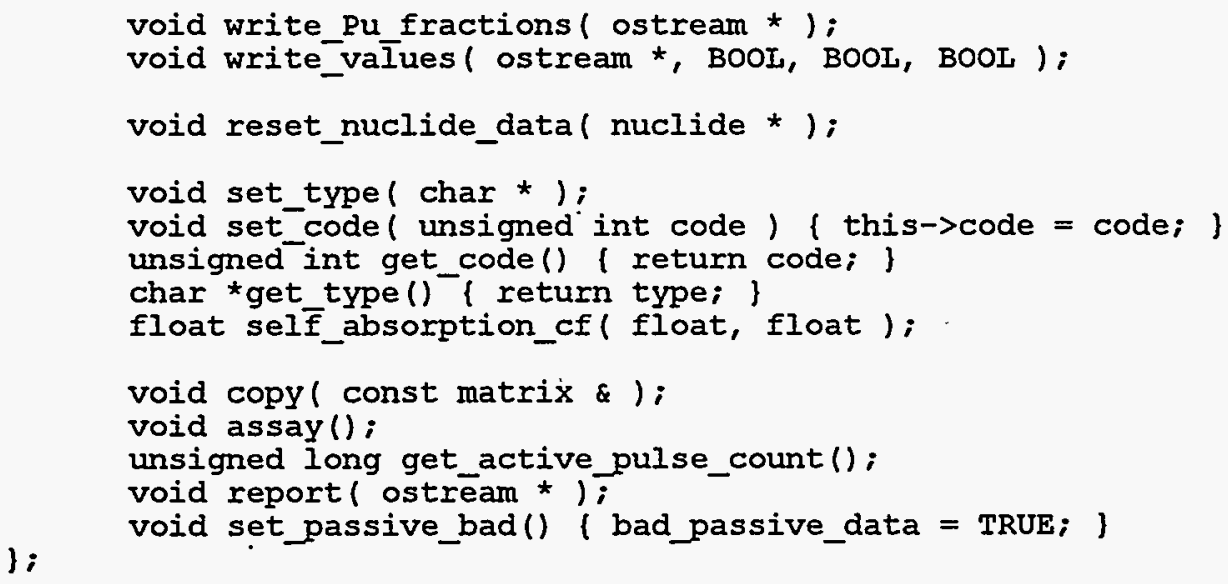

// Rev 1.0 05 Oct 1993 15:25:38 esm

// Initial release.

Title: SWEPP Assay System

Project ID: Task Baseline Agreement SDM-92-097

Developed by: Scientific Computing Unit, INEL

Funded by: Transuranic Waste Programs Unit, INEL

\subsection{Software Module}

\$Workfile: matrlist.h $\$$ \$Revision: 2.0 \$

2. Function / Purpose

The matrix list object models the list of matrixes that are processed at the assay station. It is derived from the "list" class (see list.h).

\section{Interface Description}

Functions are defined for appending, inserting, and removing matrixes. A find function searches the list for a matrix with a specified code.

4. History

Version 2.0 Release, April, 1996 (I. V. East)

User interface improvements, additional data integrity tests and additional calculational capabilities as outlined in' INEL/RWMC system Change Request No. 13623 (February, 1996). See Software Requirements Specification, INEL-96/0056, and Software Design Description, INEL-96/0057, for a detailed description of this program. 


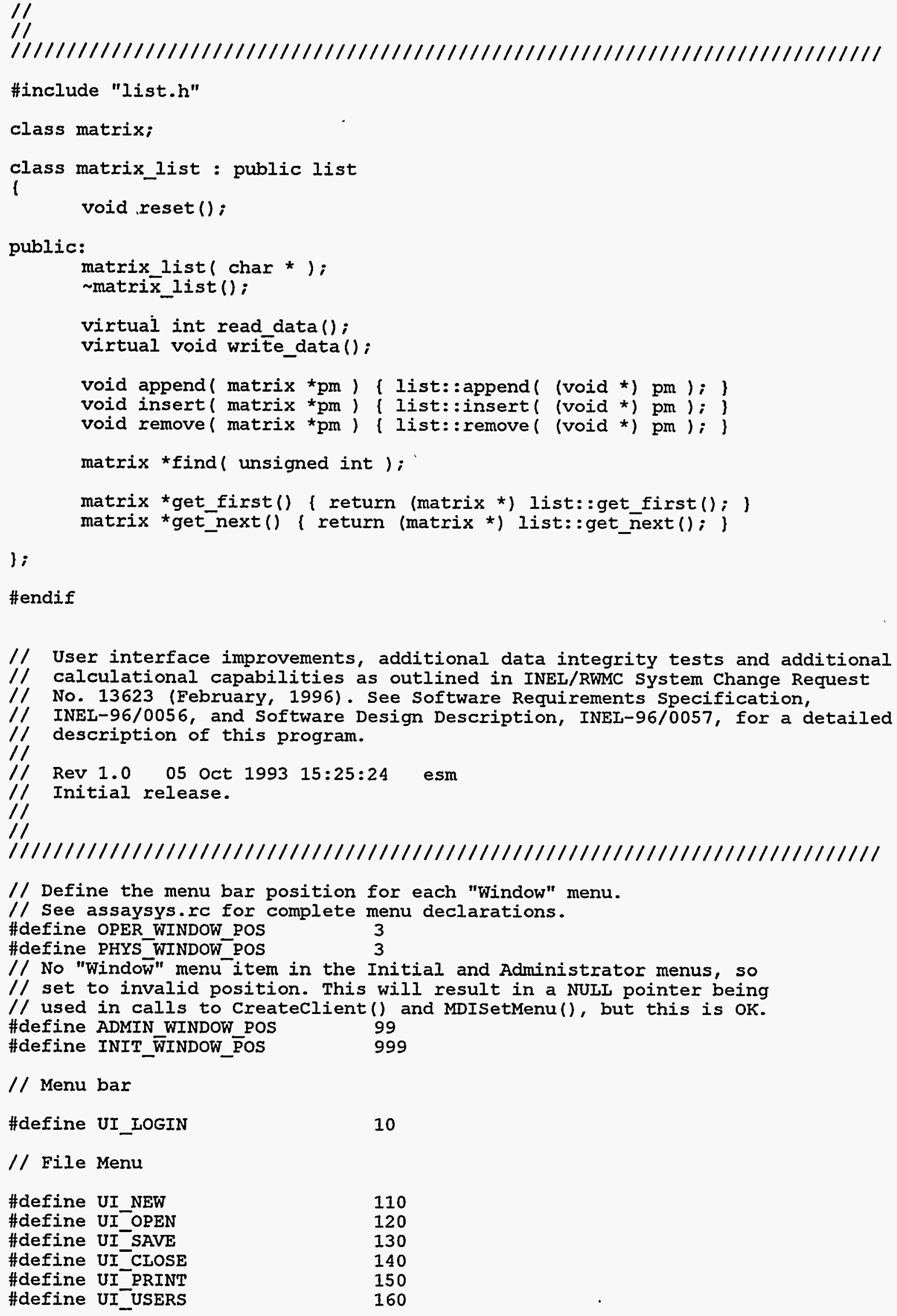

// User interface improvements, additional data integrity tests and additional

// calculational capabilities as outlined in INEL/RWMC system Change Request

// No. 13623 (February, 1996). See Software Requirements Specification,

// INEL-96/0056, and Software Design Description, INEL-96/0057, for a detailed

// description of this program.

$1 /$

// Rev 1.0 05 Oct $199315: 25: 24$ esm

// Initial release.

$1 /$

$1 /$

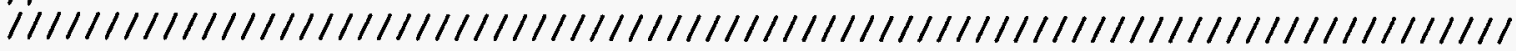

// Define the menu bar position for each "Window" menu.

// See assaysys.re for complete menu declarations.

\#define OPER_WINDOW_POS

\#define PHYS_WINDOW_POS

3

3

// No "Window" menu item in the Initial and Administrator menus, so

// set to invalid position. This will result in a NULL pointer being

// used in calls to Createclient() and MDISetMenu(), but this is OK.

\#define ADMIN WINDOW POS 99

\#define INIT_WWINDOW_P̄OS 999

// Menu bax

\#define UI_LOGIN

// File Menu

$\begin{array}{ll}\text { \#define UI_NEW } & 110 \\ \text { \#define UI_OPEN } & 120 \\ \text { \#define UI_SAVE } & 130 \\ \text { \#define UI_CIOSE } & 140 \\ \text { \#define UI_PRINT } & 150 \\ \text { \#define UI_USERS } & 160\end{array}$


INEL-96/0057

$\begin{array}{ll}\text { \#define UI_NEW_IOG } & 170 \\ \text { \#define UI_PRINT_LOG } & 180 \\ \text { \#define UI_EXIT } & 190 \\ \text { \#define UI_LOGOUT } & 20 \\ \text { // Run Menu } & \\ & \\ \text { \#define UI_ASSAY } & 210 \\ \text { \#define UI_ACTIVE } & 220 \\ \text { \#define UI_PASSIVE } & 230 \\ \text { \#define UI_BACKGROUND } & 240 \\ \text { \#define UI_VERIFICATION } & 250 \\ \text { \#define UI_CHI_SQUARE } & 260 \\ \text { \#define UI_REMOTE } & 270 \\ \text { \#define UI_REGRESSION } & 280 \\ \text { \#define UI_TEST_CRATE } & 290\end{array}$

// Options Menu

\#define UI SUMMARY REPORT 310

\#define UI_BACKGROUND_CORRECTION 320

\#define UI SET COLOR - 330

\#define UI_SET_DEFAULTS 340

\#define UI CAIIBRATION 350

\#define UI_CONTAINER 360

\#define UI_MATRIX 370

// Window Menu

\#define UI CASCADE

\#define UI_TILE 420

\#define UI NEXT 430

\#define UI ARRANGE $\quad 440$

// Help Menu

\#define UI_ABOUT 990

// Non-menu commands

\#define DO_ASSAY 9010

\#define DO RECALC 9020

\#define END_REMOTE 9030

\#define PRINTT REPORT 9040

\#define CONTIÑNE 9050

\#define ACCEPT_BKGND 9060

\#define REJECT BKGND 9070

\#ifndef _INC_message_dlg_

\#define _INC_message_dlg_

$/ / / / / / / / / / / / / / / / / / / / / / / / / / / / / / / / / / / / / / / / / / / / / / / / / / / / / / / / / / / / 1$

11

// 1. Identification

// 1.1 Project

$1 /$

$1 /$

11

11

// Written October 1995 by I. V. East as part of. V2.0 modifications. 
// 2. Function / Purpose

Dialog box to display a message to user. Intended for use when bad data or other problem is encountered and allow user to choose to about current operation.

3. Interface Description

Derived from the Microsoft Foundation Class CDialog.

4. History

Version 2.0 Release, April, 1996 (I. V. East)

User interface improvements, additional data integrity tests and additional calculational capabilities as outlined in INEL/RWMC system Change Request No. 13623 (February, 1996). See Software Requirements Specification,

INEL-96/0056, and Software Design Description, INEL-96/0057, for a detailed description of this program.

// Rev 1.013 Oct 1995 lve

// Initial implementation.

$1 /$

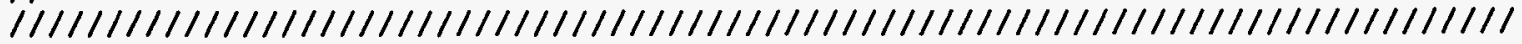

class message_dlg : public CDialog

1

private:

char *title;

char *message;

public:

message_dlg( char *, char * );

rmessage_dlg();

BOOL OnInitDialog();

afx_msg void onDestroy();

l;

DECLARE_MESSAGE_MAP ()

\#endif // _INC_message_dlg_

\#ifndef INC_nuclide_

\#define INC_nuclide_-

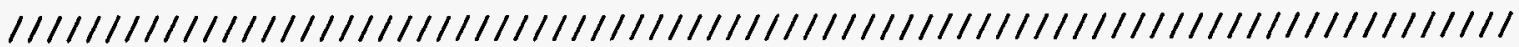

$1 /$

// 1. Identification

$1 /$

11

$1 /$

$1 /$

1.1 Project

Title: SWEPP Assay System

Project ID: Task Baseline Agreement SDM-92-097

Developed by: Scientific Computing Unit, INEL

Funded by: Transuranic Waste Programs Unit, INEL

\subsection{Software Module}

\$Workfile: nuclide.h $\$$

\$Revision: 2.0 \$ 


\section{INEL-96/0057}

$/ /$

//2. Function / Purpose

11

The nuclide class encapsulates the nuclide data required for assay analysis It also includes the calculated nuclide mass (value $+/$ - uncertainty).

3. Interface Description

\section{History}

Version 2.0 Release, April, 1996 (I. V. East)

User interface improvements, additional data integrity tests and additional calculational capabilities as outlined in INEL/RWMC System Change Request No. 13623 (February, 1996). See Software Requirements Specification, INEL-96/0056, and Software Design Description, INEL-96/0057, for a detailed description of this program.

Version 2 modifications -- December 1995 Ive Class members "mass ratio", "aux ratio" "equivalent Pu factor", "fissile_gramiequivālent", "x_sečtion" and "nu_bar"-ad̄ed.

Removed unused constructors.

All "float" member variables initialized to zero in constructor.

Rev $1.0 \quad 05$ Oct $199315: 25: 40$ esm

Initial release.

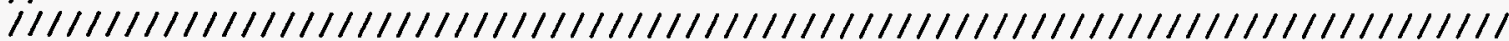

\#include "errorbar.h"

class nuclide

l

public:

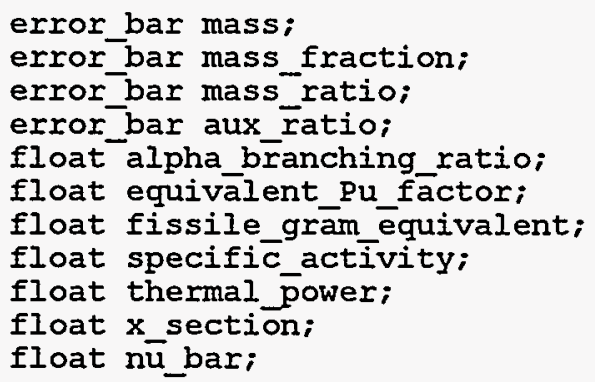

// Calculated value.

$1 / 0.0$ to 1.0

// Mass ratio relative to $\mathrm{Pu}-239$ (usually...)

// Mass ratio relative to something else

// 0.0 to 1.0

// Weighting factor for $\mathrm{Pu}$ equivalent act.

// Positive value; relative to 1.0 for $\mathrm{Pu}-239$

// Total Curies per gram (Ci/g)

// Watts per gram (Watts/g)

// (Effective) fission cross-section (barns)

// Average number of neutrons/fission 
specific_activity $=$ thermal_power $=x \_$section $=$nu_bar $=0.0 f ;$ ]

):

\#endif

\#ifndef INC_passive_dlg_

\#define INC_passive_dlg_

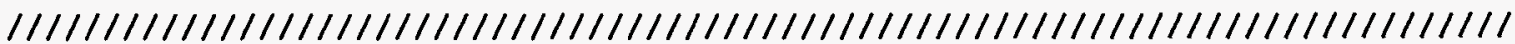

$1 /$

// 1. Identification

$1 /$

$1 /$

11

$1 /$

1.1 Project

Title: SWEPP Assay System

Project ID: Task Baseline Agreement SDM-92-097

Developed by: Scientific Computing Unit, INEL

Funded by: Transuranic Waste Programs Unit, INEL

1.2 Software Module

\$Workfile: pasdlg.h $\$$

\$Revision: 2.0 \$

2. Function / Purpose

The passive dialog provides the user interface to view the counts and rates from a passive data acquisition.

3. Interface Description

11

$1 /$

$1 /$

// 4. History

$1 /$

// Version 2.0 Release, April, 1996 (L. V. East)

// User interface improvements, additional data integrity tests and additional

// calculational capabilities as outlined in INEL/RWMC system Change Request

// No. 13623 (February, 1996). See Software Requirements Specification,

// INEL-96/0056, and Software Design Description, INEL-96/0057, for a detailed

// description of this program.

//

// Rev 1.005 Oct $1993 \quad 15: 25: 36$ esm

// Initial release.

$1 /$

11

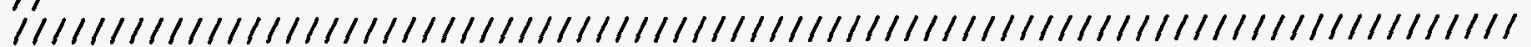

\#define MAX_PASSIVE_BADVALUES

24

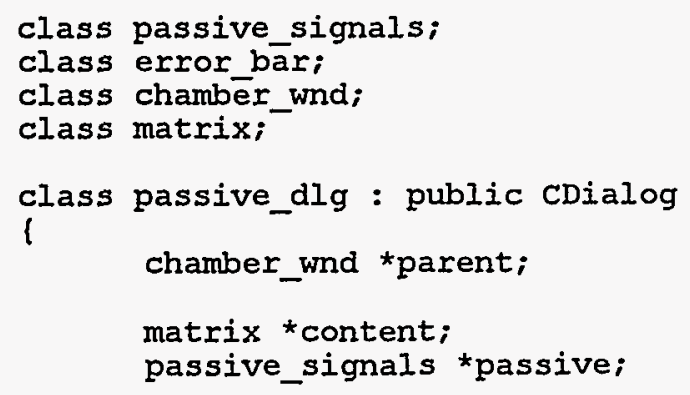


BOOL background_run;

// will be TRUE only for passive background runs.

char buffer[ RECORD_LENGTH ];

HBRUSH hbrBkgnd; // Background brush to use for highlighted message boxes. COIORREF bkgColor; // Color to use for background brush.

BOOL bad counts;

BOOL bad rate;

int bad_valueIDs [ MAX_PASSIVE_BADVALUES ];

int bad values;

float fuzzy;

unsigned long set_count ( $\operatorname{char}$ *, int );

unsigned long set count ( unsigned long, int);

void set_rate( error_bar, int, int );

afx_msg HBRUSH OnCtlColor( CDC*, CWnd*, UINT );

afx msg void onclose ();

afx_msg void onLButtonDown( UINT, CPoint );

afx msg void onpaint () ;

afx msg void on accept ();

afx_msg void on_reject();

public:

passive_dlg( passive_signals *, chamber_wnd *,

passive dlg():

matrix *content $=\overline{\text { NULL}}$, BOOL bkgnd_run $=$ FALSE );

void rate check();

void enable_buttons ():

BOOL OnInitDialog();

);

DECLARE_MESSAGE_MAP()

\#endif

\#ifndef INC_passive_signals

\#define -INC_passive_signals_

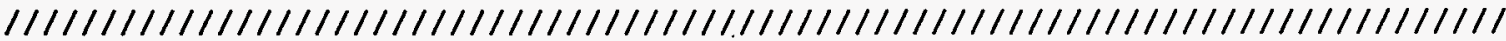

$1 /$

// 1. Identification

$1 /$

$1 /$

1.1 Project

Title: SWEPP Assay system

Project ID: Task Baseline Agreement SDM-92-097

Developed by: Scientific Computing Unit, INEL

Funded by: Transuranic Waste Programs Unit, INEI

1.2 Software Module

\$Workfile: passgnls.h \$

\$Revision: 2.0 \$

2. Function / Purpose

The passive signals provides the interface to the passive data to access the shielded total, the system total, the shielded coincidence, and the system coincidence signals. 


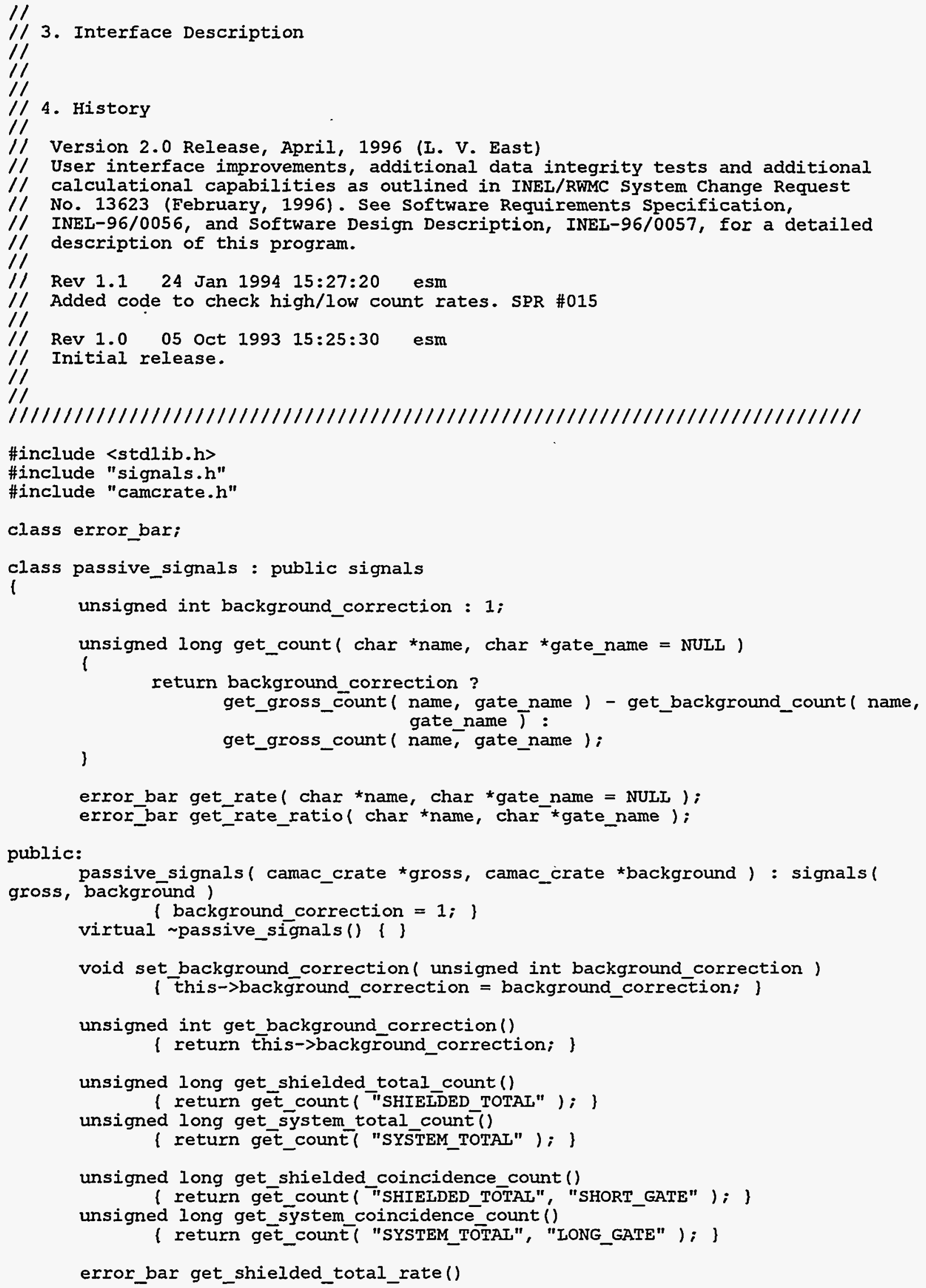




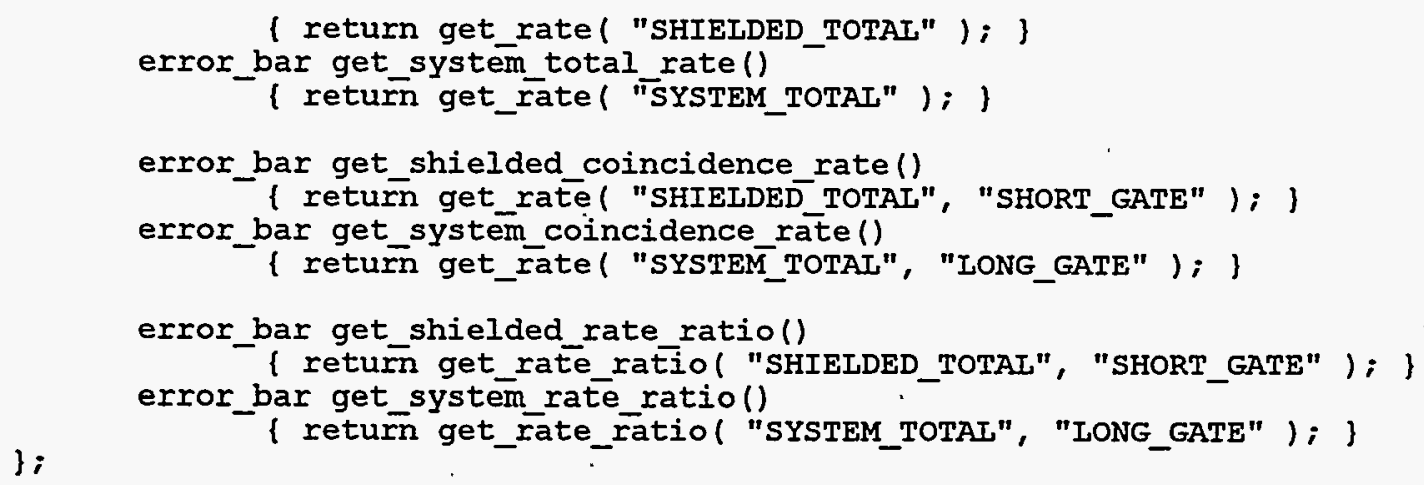

1.1 Project

Title: SwEPP Assay system

Project ID: Task Baseline Agreement SDM-92-097

Developed by: Scientific Computing Unit, INEL

Funded by: Transuranic Waste Programs Unit, INEL

\subsection{Software Module}

\$Workfile: passgnls.h \$ \$Revision: $2.0 \$$

2. Function / Purpose

The passive signals provides the interface to the passive data to access the shielded total, the system total, the shielded coincidence, and the system coincidence signals.

3. Interface Description

\section{History}

Version 2.0 Release, April, 1996 (I. V. East)

User interface improvements, additional data integrity tests and additional calculational capabilities as outlined in INEL/RWMC system Change Request No. 13623 (February, 1996). See Software Requirements Specification, INEL-96/0056, and Software Design Description, INEL-96/0057, for a detailed description of this program.

Rev 1.1 24 Jan 1994 15:27:20 esm Added code to check high/low count rates. SPR \#015

Rev 1.0 05 Oct 1993 15:25:30 esm Initial release.

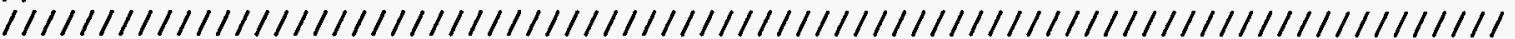




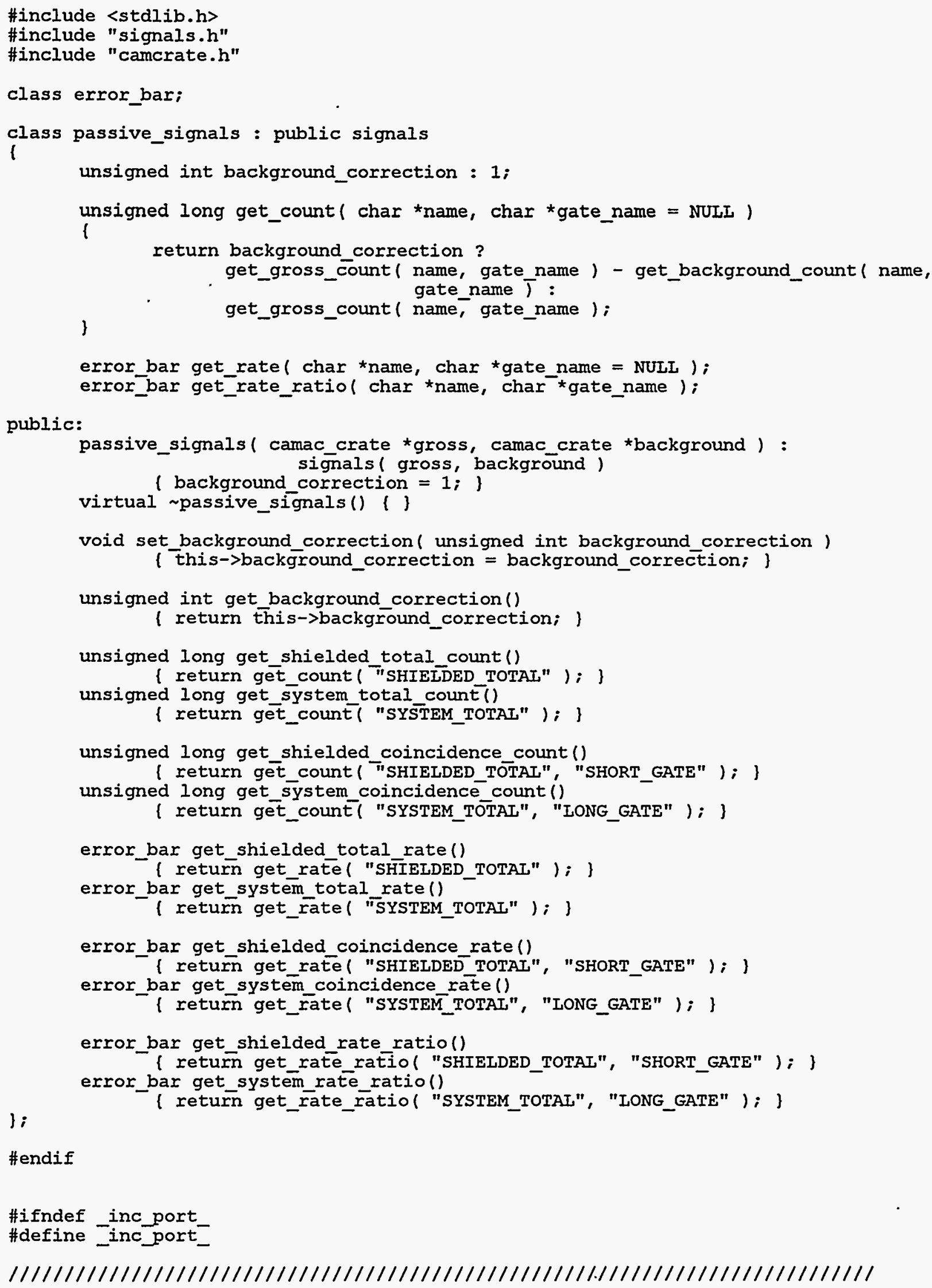




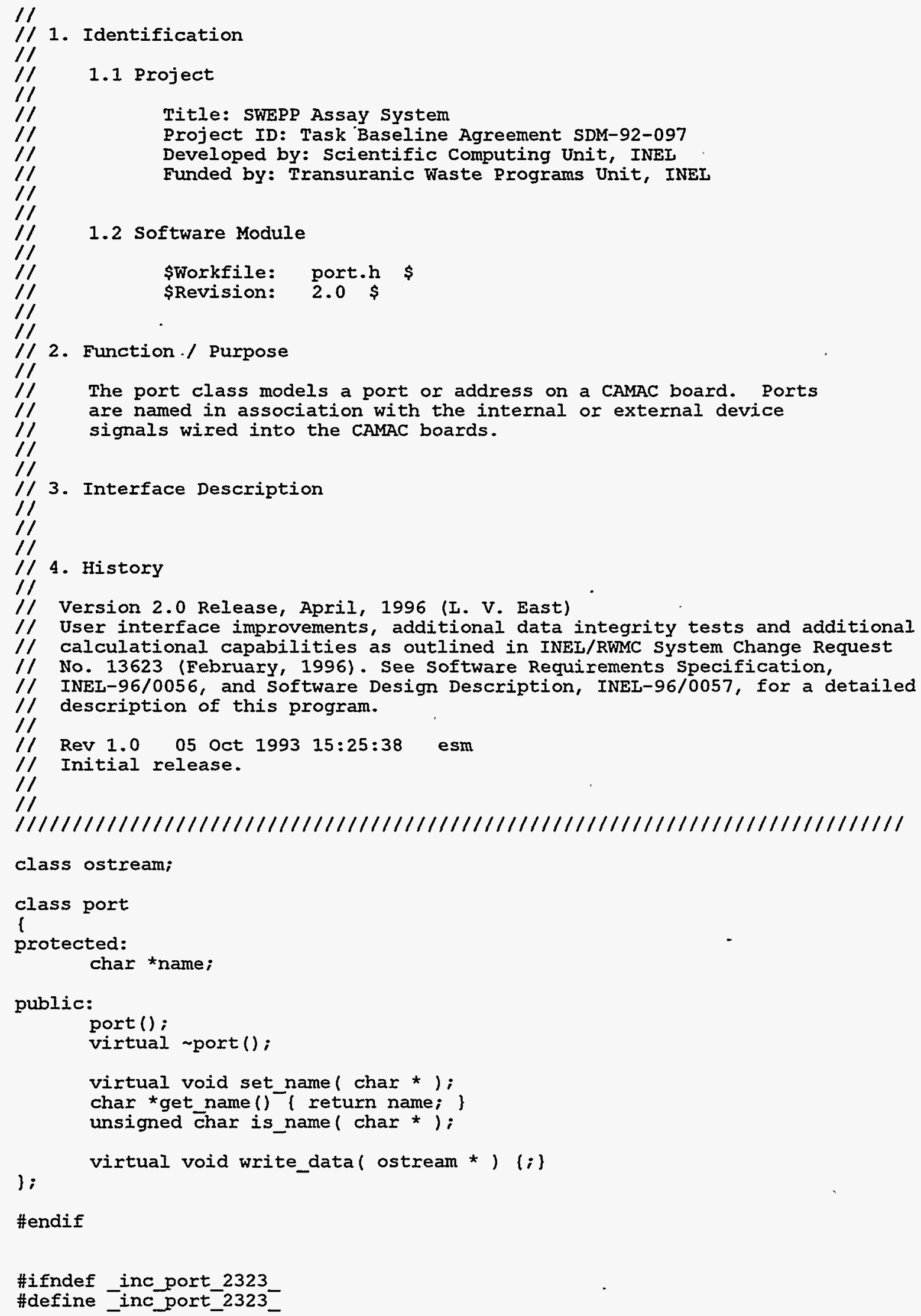


// 1. Identification

1.1 Project

Title: SWEPP Assay System

Project ID: Task Baseline Agreement SDM-92-097

Developed by: Scientific Computing Unit, INEL

Funded by: Transuranic Waste Programs Unit, INEL

\subsection{Software Module}

\$Workfile: port2323.h \$

\$Revision: 2.0 \$

2. Function / Purpose

// The port_2323 class models the ports on a LeCroy 2323 Dual Gate and

// Delay Geñerator.

$1 /$

// 3. Interface Description

$1 /$

$1 /$

$1 /$

// 4. History

//

// Version 2.0 Release, April, 1996 (L. V. East)

// User interface improvements, additional data integrity tests and additional

// calculational capabilities as outIined in INEL/RWMC system Change Request

// No. 13623 (February, 1996). See Software Requirements specification,

// INEL-96/0056, and Software Design Description, INEL-96/0057, for a detailed

$/ /$. description of this program.

$1 /$

// Rev $1.0 \quad 05$ Oct $199315: 25: 36$ esm

// Initial release.

$1 /$

$1 /$

// 5. References

$1 /$

// 5.1 Operator's Manual, CAMAC Model 2323A, Dual Gate and Delay Generator

$1 /$ Lecroy, May 1987

$1 /$

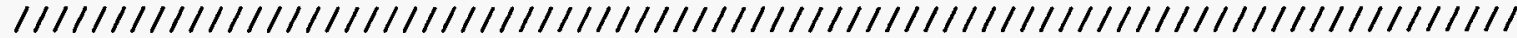

// The programming of each port is managed through a 16 bit word that is

// partitioned into 2 bits for delay, 1 bit for latch, 3 bits for exponent,

$/ /$ and 10 bits for the mantissa. The internal delay may be set for 10ns, 30ns,

// I00ns, or 300ns. The latch determines the mode, whether internal

// or external. The exponent and mantissa define the gate width.

$$
\text { \#define DELAY_10ns } 0 \times 00 / / \text { delay bits - defines delay width }
$$

\#define DELAY 30ns 0x01

\#define DELAY I00ns 0x02

\#define DELAY 300ns 0x03

\#define MIN_DẼLAY DELAY IOns

\#define MAX_DELAY DELAY_300ns

\#define INTERNAL $0 \times 00 / /$ latch bit = 0; gate duration defined by control word \#define EXTERNAI 0x01 // latch bit = 1; gate duration defined by START and STOP 


\section{INEL-96/0057}

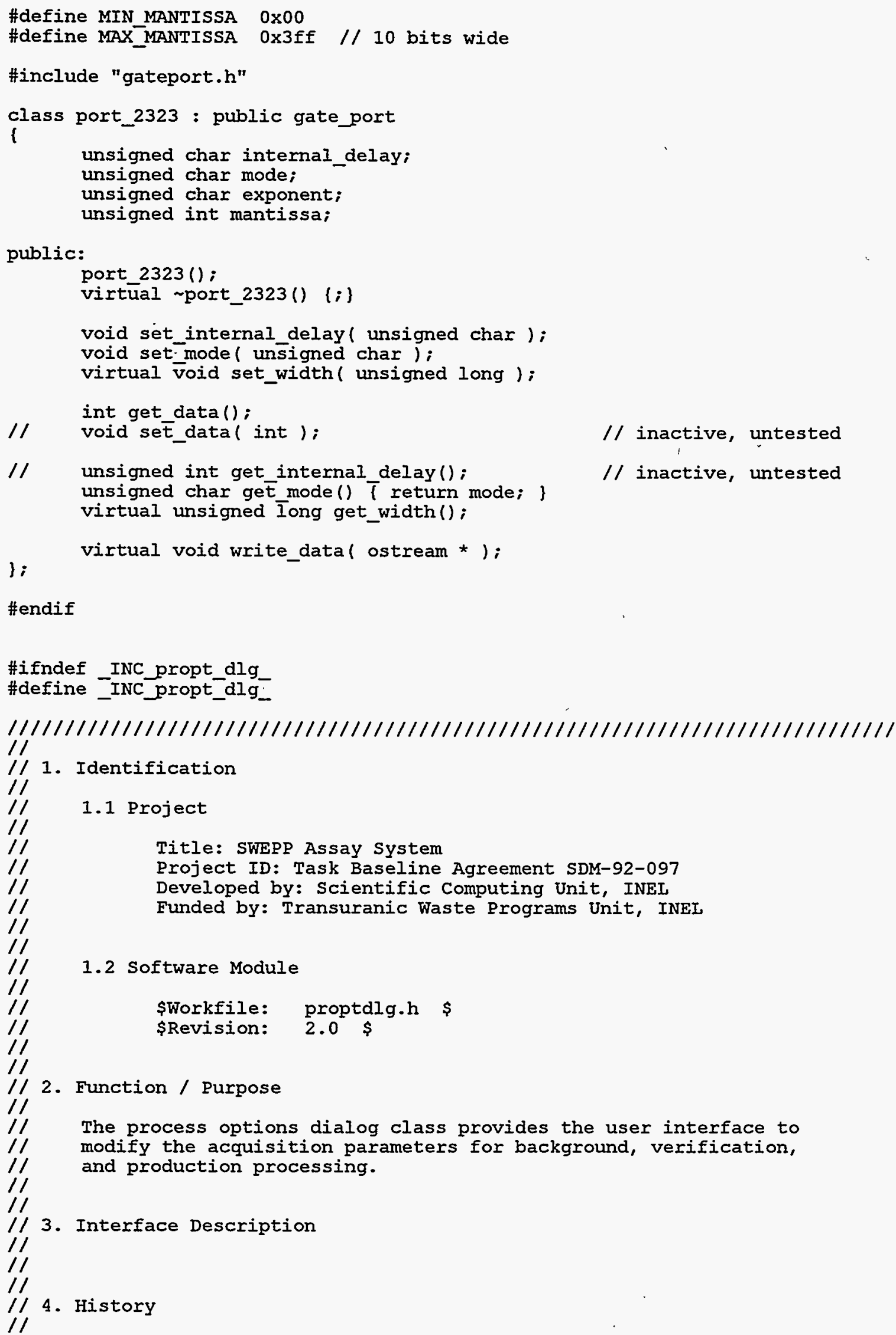

// 2. Function / Purpose

// 3. Interface Description

$1 /$

11

$1 /$

// 4. History

1.1 Project

Title: SWEPP Assay system

Project ID: Task Baseline Agreement SDM-92-097

Developed by: Scientific Computing Unit, INEL

Funded by: Transuranic Waste Programs Unit, INEI

1.2 Software Module

\$Workfile: proptdlg.h \$

\$Revision: 2.0 \$

The process options dialog class provides the user interface to modify the acquisition parameters for background, verification, and production processing. 
// Version 2.0 Release, April, 1996 (I. V. East)

// User interface improvements, additional data integrity tests and additional

// calculational capabilities as outlined in INEL/RWMC system Change Request

// No. 13623 (February, 1996). See Software Requirements specification,

// INEL-96/0056, and Software Design Description, INEL-96/0057, for a detailed

$/ /$ description of this program.

//

// Rev 1.0 05 oct 1993 15:25:24 esm

// Initial release.

$1 /$

$1 /$

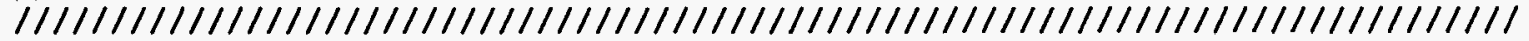

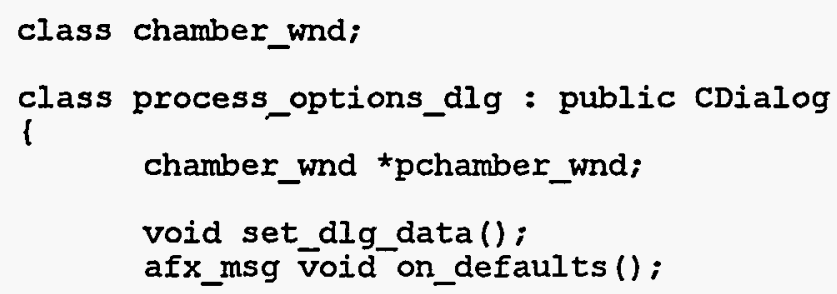

// 2. Function / Purpose

// 3. Interface Description

Title: SWEPP Assay System Project ID: Task Baseline Agreement SDM-92-097 Developed by: Scientific Computing Unit, INEI Funded by: Transuranic Waste Programs Unit, INEL

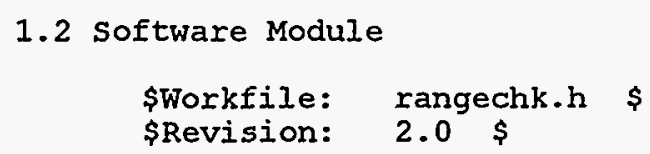

$1 /$ 


\section{INEL-96/0057}

// Version 2.0 Release, April, 1996 (L. V. East)

// User interface improvements, additional data integrity tests and additional

// calculational capabilities as outlined in INEL/RWMC system Change Request

// No. 13623 (February, 1996). See Software Requirements Specification,

$/ /$ INEL-96/0056, and Software Design Description, INEL-96/0057, for a detailed

// description of this program.

30 June 95 Changed to use "ASSERT" (MFC macro) rather than "assert". /Ive Rev $1.0 \quad 05$ oct $1993 \quad 15: 25: 22$ esm Initial release.

11

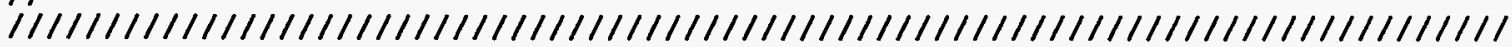

\#include $\langle a f x . h\rangle$

\#define RANGE_CHECK( $\min$, max, value) ASSERT( $\min <=$ value \&\& value $<=\max$ ) \#endif

\#ifndef INC_results_dlg
\#define _INC_results_dlg_

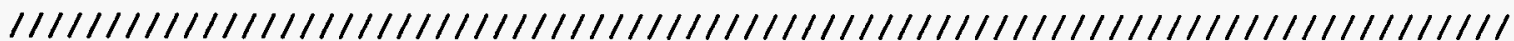

$1 /$

// 1. Identification

/I

// 3. Interface Description

1.1 Project

Title: SwEPp Assay system

Project ID: Task Baseline Agreement SDM-92-097

Developed by: Scientific Computing Unit, INEL

Funded by: Transuranic Waste Programs.Unit, INEL

\subsection{Software Module}

\$Workfile: resltdlg.h $\$$

2. Function / Purpose

The results dialog provides the user interface to view the results of the assay calculations.

\section{History}

Version 2.0 Release, April, 1996 (I. V. East)

User interface improvements, additional data integrity tests and additional calculational capabilities as outlined in INEL/RWMC system Change Request No. 13623 (February, 1996). See Software Requirements Specification, INEL-96/0056, and Software Design Description, INEL-96/0057, for a detailed description of this program.

Rev 1.0 05 oct 1993 15:25:36 esm

Initial release. 


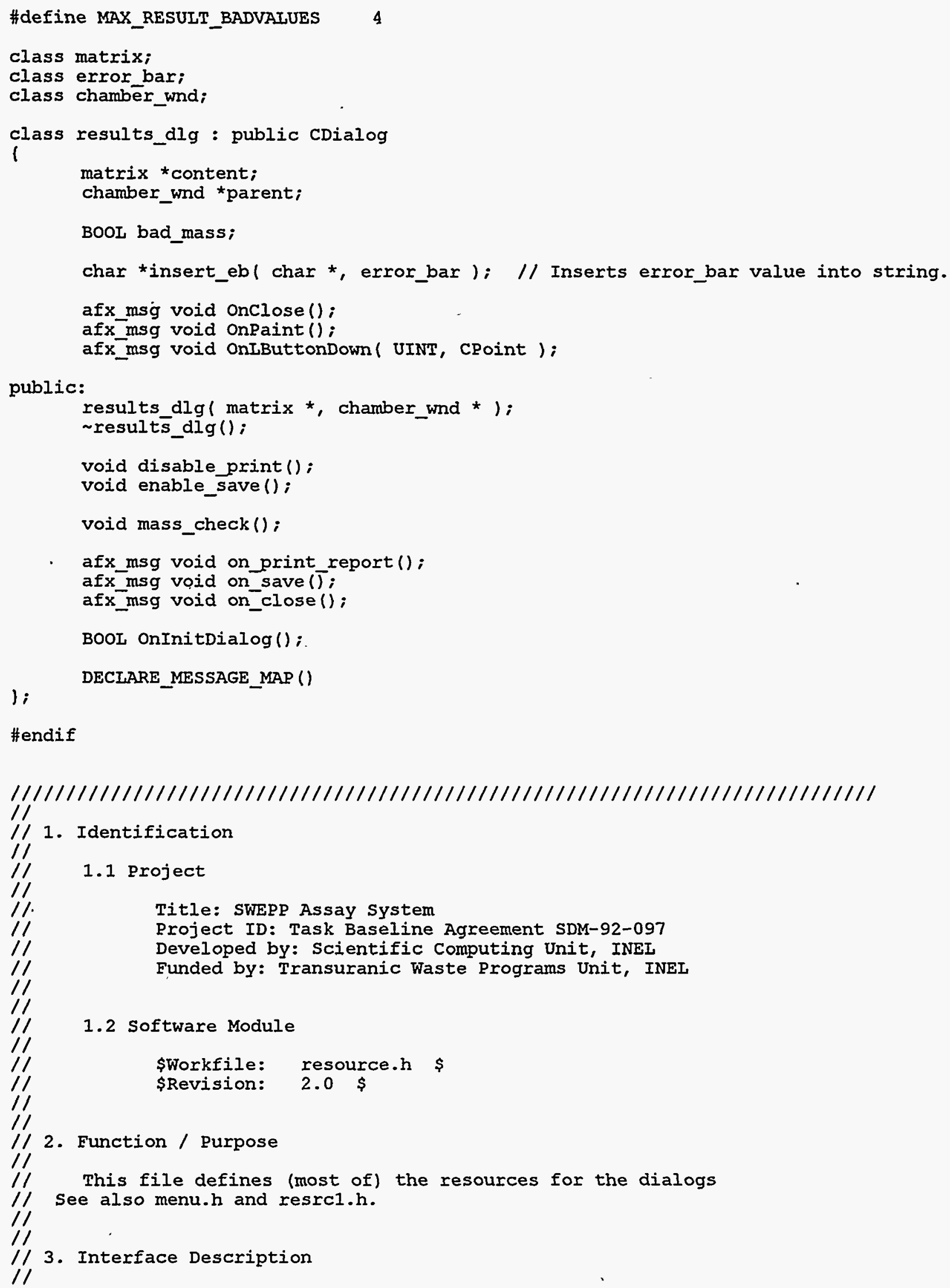

/1

11. Title: SWEPp Assay system

// Project ID: Task Baseline Agreement SDM-92-097

// Developed by: Scientific Computing Unit, INEL

// Funded by: Transuranic Waste Programs Unit, INEL

$1 /$

$1 /$

// 1.2 Software Module

11

// \$Workfile: resource.h $\$$

/1 \$Revision: 2.0 \$

$1 /$

$1 /$

// 2. Function / Purpose

11

// This file defines (most of) the resources for the dialogs

// See also menu.h and resrc1.h.

II

11

// 3. Interface Description

II 


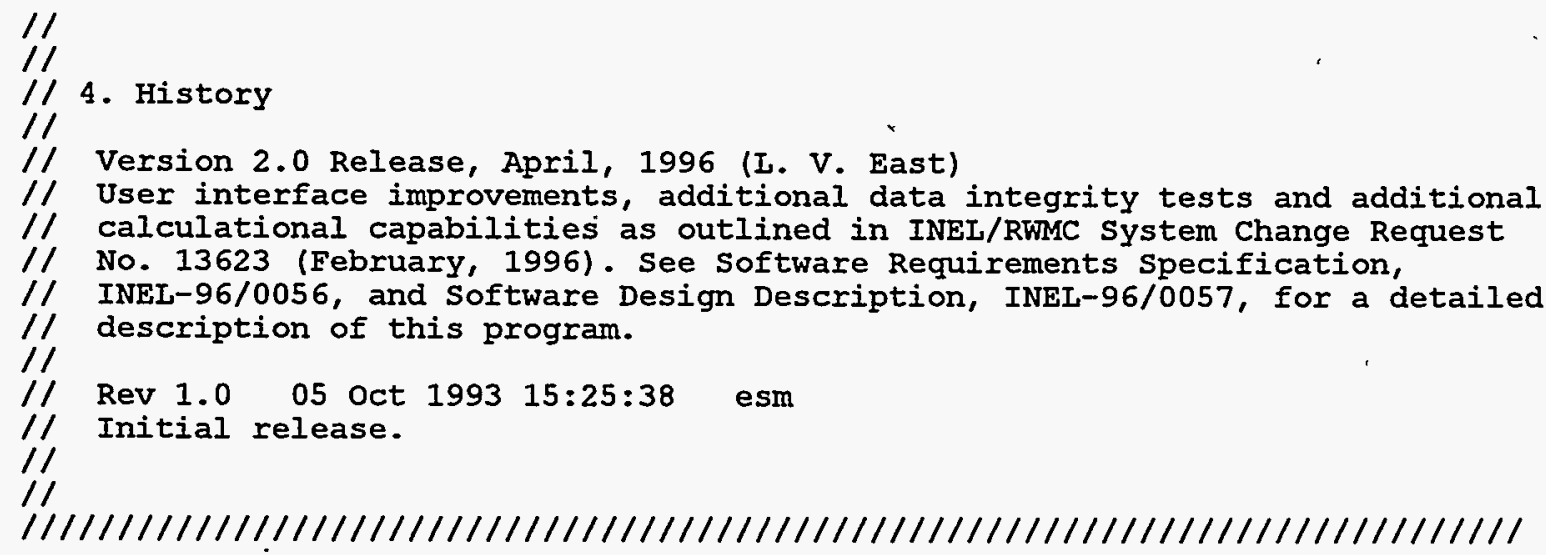


\#define ID C3.

\#define ID BKG SECS

\#define IDD_ABOUT 1300

\#define ID_SHIELDED_COINCIDENCE 608

\#define ID_SHIEIDED COINCIDENCE ERROR 609

\#define ID_SYSTEM_COINCIDENCE - 610

\#define ID_SYSTEM_COINCIDENCE_ERROR 611

\#define ID NAME - 1401

\#define ID_VOLUME 1403

\#define ID $\overline{\bar{L}}$ CONTAINERS 1405

\#define IDB_ADD 1406

\#define IDB_CHANGE 1407

\#define IDB_DELETE 1408

\#define IDI_MATRIX $\quad 715$

\#define IDD_MATRIX_PARAMETERS 1600

\#define ID_M M _- 1601

\#define ID_DIV 1602

\#define IDD_MATRIX_LIST $\quad 700$

\#define IDD_CONTAIN̄ER_IIST 1400

\#define IDD_WASTE_ID - 1100

\#define IDCB_CONTĀINER 1101

\#define IDCB MATRIX 1102

\#define IDT_TEXT 1702

\#define IDT_TEXT2 1703

\#define ID_BARE_LEFT 102

\#define ID_SHIEIDDED RATE ERROR 504

\#define ID_ELUX_RATE_ERROR

\#define ID_BARRẼL_RATTE_ERROR 506

\#define ID_BARE_RIGHT - 508

\#define ID_BARE_DOOR 510

\#define ID_BARE_TOP 511

\#define ID_BARE_BOTTOM 512

\#define ID_SHIETDDED_LEFT

\#define ID_SHIELDED_BACK

\#define ID_SHIELDED_RIGHT

\#define ID_SHIELDED_DOOR 516

\#define ID_SHIELDED_TOP 517

\#define ID_SHIELDED_BOTTOM 518

\#define ID_GROSS_SHIEIDED 520

\#define ID_GROSS_FLUX 521

\#define ID_GROSS_BARREL 522

\#define ID_SYSTEM_RATE

\#define ID_SYSTEM_RATE_ERROR 541

\#define ID_SHIELDED_RATE

\#define ID_FLUX_RATE $\bar{E}$

\#define ID_BARRĒL_RATE 548

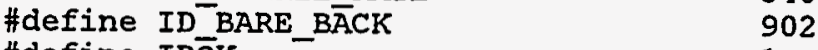

\#define IDŌK _ 1

\#define ID_POWER $\quad 527$

\#define ID_POWER DENSITY 528

\#define ID_ACTIVITY 529

\#define ID_ACTIVITY_CONCENTRATION 530

\#define ID_PU_MASS - 531

\#define ID_AM-MASS_ERROR 532

\#define ID_POWER_ĒRROR 1001

\#define ID_POWER_DENSITY ERROR 1002

\#define ID_ACTIVITY_ERROR $\bar{R}$

\#define ID_ACTIVITY_CONCENTRATION_ERROR 1004

\#define ID_PU_MASS_ERROR 1005

\#define ID_AM_MASS $\quad 1007$

\#define ID_BACKG_BARREL

\#define ID_BACKG_SHIELDED 524

\#define ID_BACKG_FLUX 525

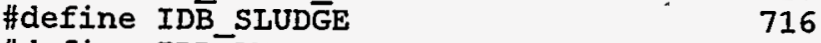

\#define IDB_AM_SLUDGE $\quad 717$

\#define ID_SYST̄EM $\quad 602$

\#define ID_SYSTEM_ERROR 603 


\section{INEL-96/0057}

\begin{tabular}{|c|c|c|}
\hline \#define & ID_BARE_TOTAL & 901 \\
\hline \#define & ID_SHIEIDED_TOTAL & 903 \\
\hline \#define & ID_PU_238 & 606 \\
\hline \#define & ID_PU_239 & 607 \\
\hline \#define & ID_PU_240 & 612 \\
\hline \#define & ID_PU־ 241 & 613 \\
\hline \#define & ID_PU_242 & 614 \\
\hline \#define & ID PASSSIVE SYSTEM & 603 \\
\hline define & ID_PASSIVE_SYSTEM_ERROR & \\
\hline define & ID_PASSIVE_SHIELDED & 0 \\
\hline efine & ID_PASSIVE_SHIELDED_ERROR & 0 \\
\hline efine & IDD_CHI_SQŪARE & 500 \\
\hline definé & 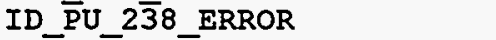 & 615 \\
\hline define & ID_PU_239_ERROR & 616 \\
\hline define & ID_PU_240_ERROR & 617 \\
\hline define & ID_PU_241_ERROR & 618 \\
\hline lefine & ID_PU_242_ERROR & 619 \\
\hline lefine & IDI_USERS'- & 201 \\
\hline \#define & IDB SAVE & 100 \\
\hline \#define & IDBCLOSE & 101 \\
\hline \#define & IDB_PRINT & 101 \\
\hline \#define & IDB_CONTINUE & 110 \\
\hline \#define & IDT_STATUS & 401 \\
\hline \#define & IDD USER IIST & 180 \\
\hline \#define & IDB_SEND_RESULTS & 40 \\
\hline \#define & IDB SEND CANCEI & 40 \\
\hline efine & IDB_EXIT_REMOTE & 40 \\
\hline fine & IDT_PROGRAMMID & 701 \\
\hline fine & ID_BARE_SUM & 904 \\
\hline lefine & ID_SHIEIDED_SUM & 905 \\
\hline efine & ID $\bar{B} \_$DEFAULTS & 12 \\
\hline
\end{tabular}

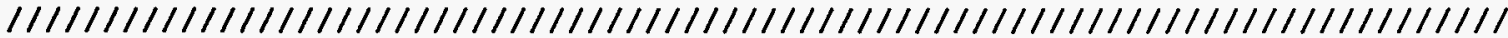
$1 /$

// 1. Identification

$1 /$

// 1.1 Project

11

//

$1 /$

/1

$1 /$

// 2. Function / Purpose

$1 /$

This file contains resource definitions generated by

Title: SWEPP Assay System

Project ID: Task Baseline Agreement SDM-92-097

Developed by: Scientific Computing Unit, INEL

Funded by: Transuranic Waste Programs Unit, INEI

\subsection{Software Module}

\$Workfile: resrcl.h $\$$

\$Revision: 2.0 \$

Microsoft Appstudio. See also menu.h and resrcl.h.

// 3. Interface Description

11

$1 /$

$1 /$

// 4. History

$1 /$

// Version 2.0 Release, April, 1996 (L. V. East)

11

$/ / / / / / / / / / / / / / / / / / / / / / / / / / / / / / / / / / / / / / / / / / / / / / / / / / / / / / / / / / / / / / / 1$ 
$/ /$ ( (NO_DEPENDENCIES\})

// App studio generated include file.

// Used by ASSAYSYS.RC

$1 /$

\#define IDD MSG BOX 103

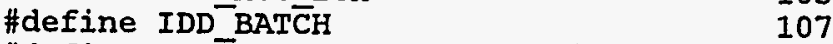

\#define IDD_AUTO 108

\#define IDD_DELAY 109

\#define IDI_TIMER 110

\#define IDC_PULSE_RATE 1205

\#define IDB_DUMMY 1207

\#define IDB_EDIT 1209

\#define IDT_RSLT_NOTE1 1212

\#define IDT RSIT NOTE2 1213

\#define ID $\bar{U}$ MAS $\bar{S}$

\#define ID_U MASS ERROR 1215

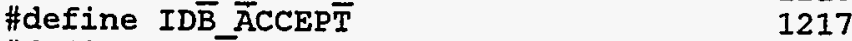

\#define IDB_REJECT 1218

\#define IDB_NON_SLUDGE 1221

\#define ID TYPE

\#define IDC̄_CHECK 1224

\#define IDC_CHECKI 1226

\#define IDB_PAUSE 1228

\#define IDB GO 1229

\#define IDC_IIST1 1232

\#define IDC-IIST2 1233

\#define IDT_RSLT_NOTE3

\#define IDT_COPYRTIGHT 1236

\#define UI_BATCH_ENABLE 40002

\#define UI_NEWF - 40003

\#define UI_AUTO ENABLE 40004

\#define UI_DATA_SAVE 40005

// Next default values for new objects

/1

\#ifdef APSTUDIO INVOKED

\#ifndef APSTUDIO_READONLY_SYMBOLS

\#define APS_NEXT_RESOURCE VAIUE 111

\#define _APS_NEXT_COMMAND_VALUE 40006

\#define _APS_NEXT_CONTROL_VALUE 1237

\#define _APS_NEXT_SYMED_VĀIUE 101

\#endif

\#endif

\#ifndef _INC_scaler

\#define _INC_scaler_

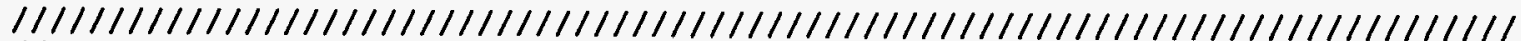

$1 / 1 / 1$

// 1. Identification

$1 /$

// 1.1 Project

//

11

11

11

11

$1 /$

$1 /$

$1 /$

11

$1 /$

//

Title: SWEPP Assay System

Project ID: Task Baseline Agreement SDM-92-097

Developed by: Scientific Computing Unit, INEL

Funded by: Transuranic Waste Programs Unit, INEL

1.2 Software Module

\$Workfile: scaler.h \$

\$Revision: 2.0 \$ 
INEL-96/0057

//2. Function / Purpose

$1 /$

The scaler class models the functionality of a generic

CAMAC scaler board.

3. Interface Description,

11

$1 /$

$1 /$

// 4. History

//

// Version 2.0 Release, April, 1996 (I. V. East)

// User interface improvements, additional data integrity tests and additional

// calculational capabilities as outlined in INEL/RWMC system Change Request

// No. 13623 (February, 1996). See Software Requirements Specification,

// INEL-96/0056, and Software Design Description, INEL-96/0057, for a detailed

$/ /$ description of this program.

// Rev 1.0 05 Oct 1993 15:25:30 esm

// Initial release.

$/ /$

$1 /$

$/ / / / / / / / / / / / / / / / / / / / / / / / / / / / / / / / / / / / / / / / / / / / / / / / / / / / / / / / / / / / / / / / 1$

\#define SCALER 301

\#include "camac.h"

class device;

class itstream;

class ostream;

class scaler : public camac

1

int has data;

int is_check_port_set;

unsigned char check_port;

void set gate name ( unsigned char, char *);

void set_clock name ( unsigned char, char *);

void set_count $\overline{(}$ unsigned char, unsigned long );

void set_device_alias ( unsigned char, char * );

protected:

virtual unsigned long read_and_clear_register( unsigned char );

virtual void clear all registers() (;)

virtual void accumūlatē_all_registers ()$,\{i\}$

virtual void clear_lam() $\{;\}$

public:

scaler ( controller $\star *$, unsigned char, unsigned int, unsigned char, unsigned char $) ;$

virtual scaler();

void set_has_data () ( has_data $=1$; \}

int has check port() ( reẼurn is check port set; )

unsigne $\bar{d}$ char get_check_port() $\{$ return check_port;

virtual unsigned long read_register( unsigned char );

device **find_device ( char *, char * );

virtual int initialize(); 
INEL-96/0057

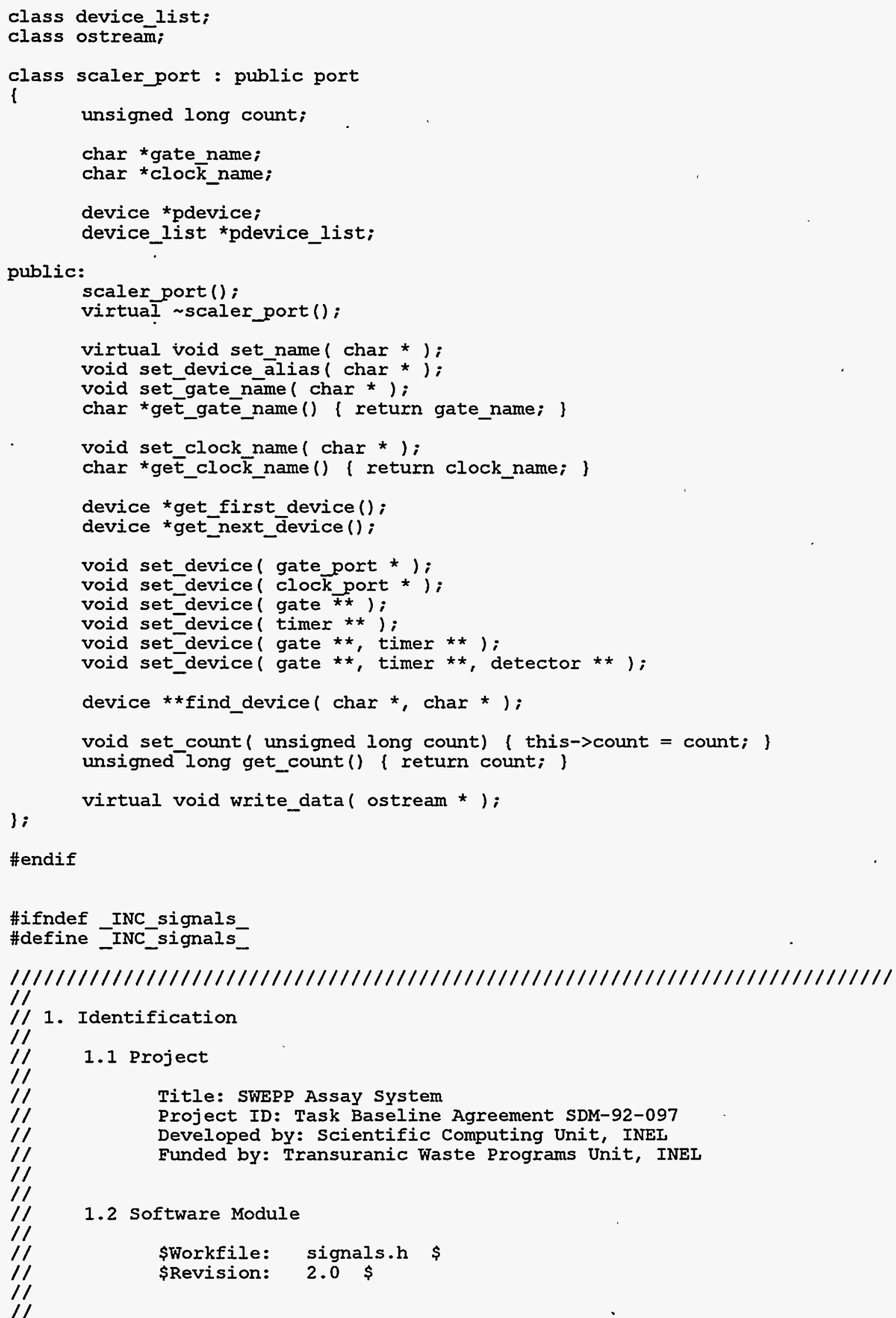

1.1 Project

Title: SwEPP Assay system

Project ID: Task Baseline Agreement SDM-92-097

Developed by: Scientific Computing Unit, INEI

Funded by: Transuranic Waste Programs Unit, INEL

1.2 Software Module

\$Workfile: signals.h \$

\$Revision: 2.0 \$ 
11

/I

II

/1

$1 /$

11

$1 /$

/I

/1

I

/I

11

II

II

/I

$1 /$

$1 /$

$1 /$

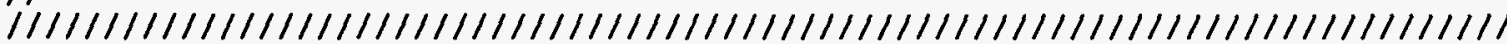

\section{Function / Purpose}

The signals class models the generic functionality to get signal rates and counts.

3. Interface Description

4. History

Version 2.0 Release, April, 1996 (L. V. East)

User interface improvements, additional data integrity tests and additional calculational capabilities as outlined in INEL/RWMC system Change Request No. 13623 (February, 1996). See Software Requirements specification, INEL-96/0056, and Software Design Description, INEL-96/0057, for a detailed description of this program.

Rev $1.0 \quad 05$ Oct $1993 \quad 15: 25: 38$ esm

Initial release.

\#include <stdlib.h>

\#include "camcrate.h"

\#include "errorbar.h"

class signals

i

camac_crate * gross;

camac_crate *background;

protected:

error_bar get_gross_rate ( char *name, char *gate_name = NULI)

( return gross->get rate ( name, gate name); )

error_bar get_background_rate ( char *name, char *gate_name = NULI )

( return background->get_rate ( name, gate_name ); )

public:

signals ( camac_crate *gross, camac_crate *background )

( this->gross = gross; this->background = background; )

virtual rignals () (;)

unsigned long get gross count ( char *name, char ${ }^{*}$ gate name $=$ NULL )

( return gross->get count ( name, gate name ); )

unsigned long get background count ( char *name, char * gate name = NULL) $($ return background->get_count ( name, gate_name );

1;

\#endif

\#ifndef INC timer

\#define -INC_timer_-

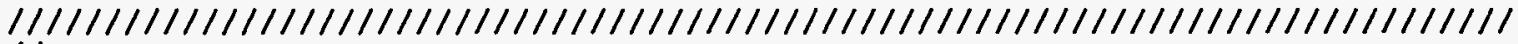

$1 /$

// 1. Identification

$1 /$

// 1.1 Project

$1 /$

11

$1 /$

Title: SWEPP Assay system

Project ID: Task Baseline Agreement SDM-92-097

Developed by: Scientific Computing Unit, INEL 


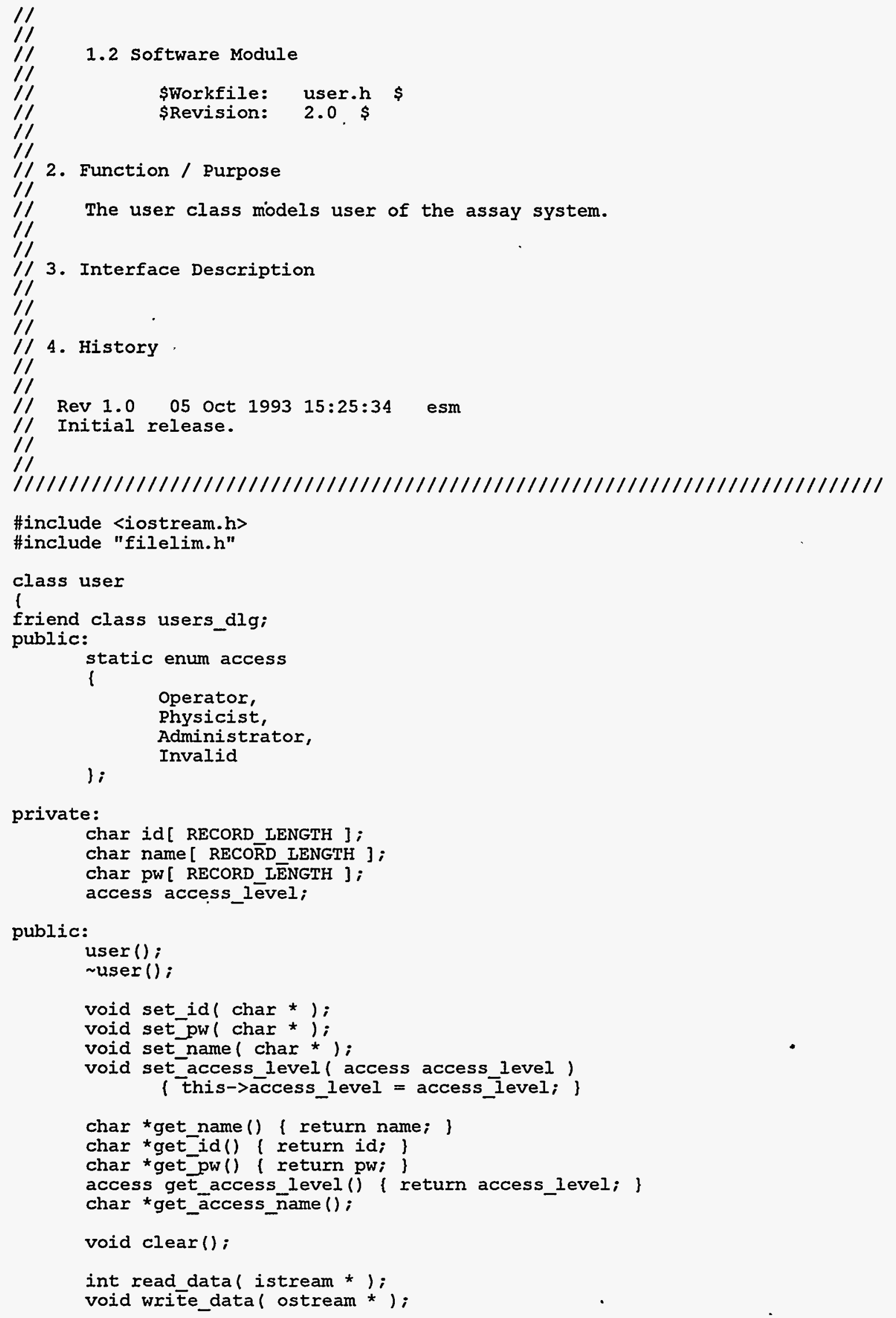


INEL-96/0057

; ;

\#endif

\#ifndef INC user list.

\#define - INC_user_list_-

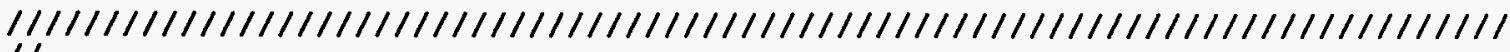

$1 /$

// 1. Identification

/1

11

II

// 3. Interface Description

// 4. History

$1 /$

// Version 2.0 Release, April, 1996 (L. V. East)

I/ User interface improvements, additional data integrity tests and additional

// calculational capabilities as outlined in INEI/RWMC system Change Request

I/ No. 13623 (February, 1996). See Software Requirements Specification,

// INEL-96/0056, and Software Design Description, INEL-96/0057, for a detailed

// description of this program.

/1

// Rev 1.0 05 Oct 1993 15:25:40 esm

// Initial release.

$1 /$

$$
11
$$

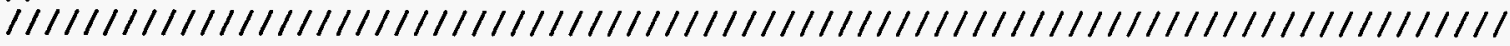

\section{\#include "list.h"}

class user;

class user_list : public list

I

void reset();

public:

user_list ( char *);

ruser Iist ();

virtual int read_data(); 


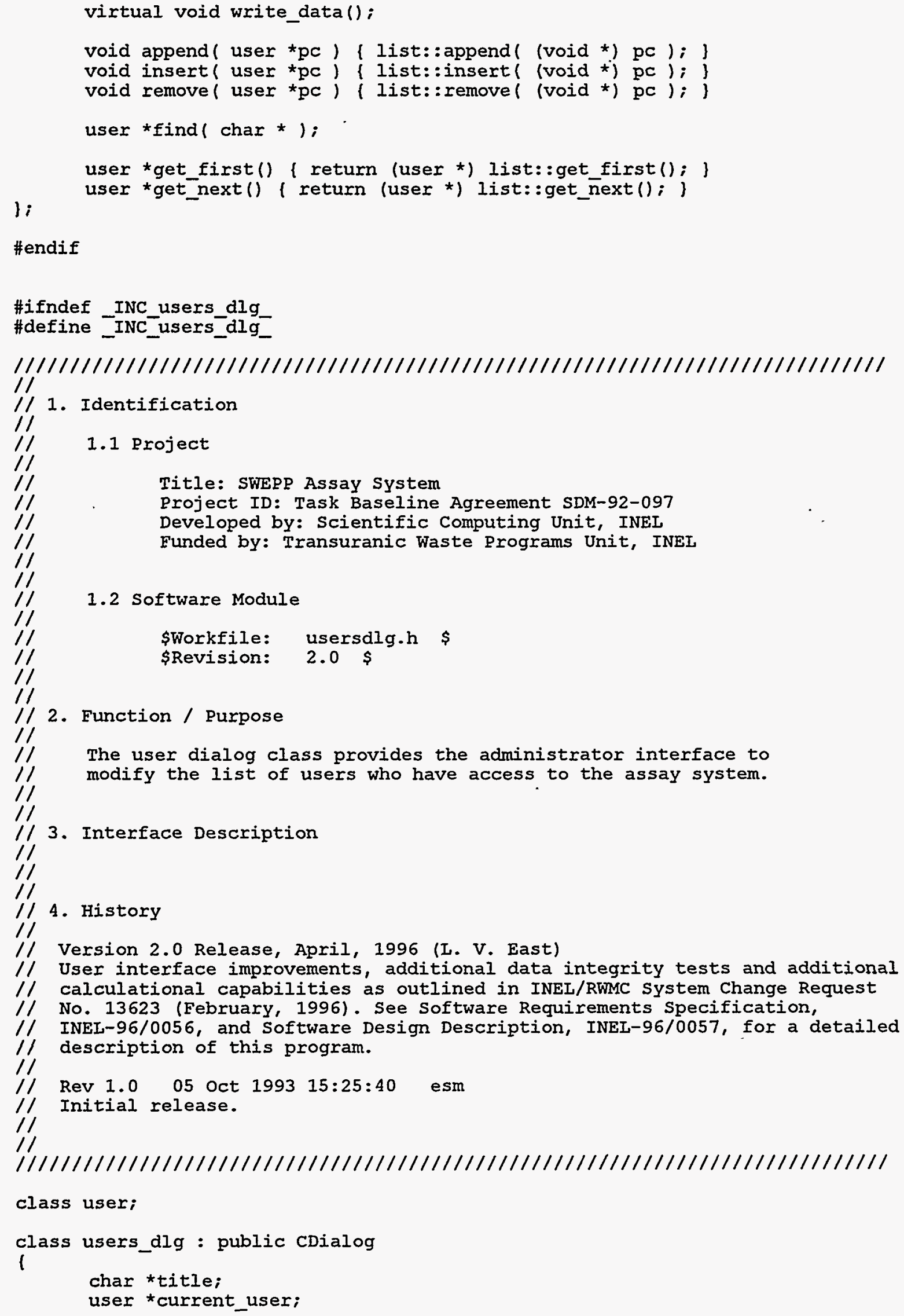

// 3. Interface Description

1.2 Software Module 


\section{INEL-96/0057}

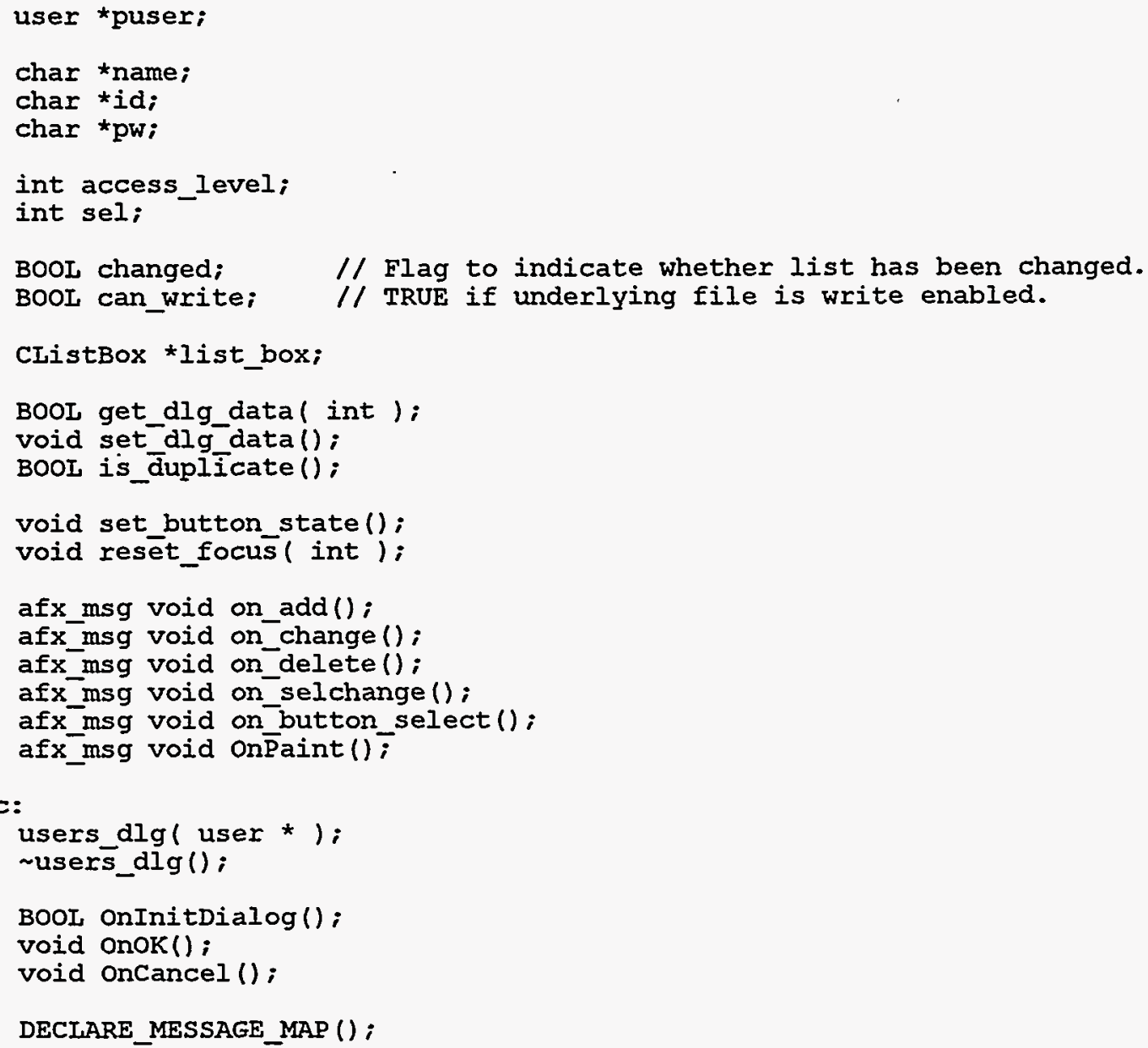

$1 /$

$1 /$

$1 /$

11

11

//2. Function / Purpose

1.1 Project

Title: SWEPP Assay System

Project ID: Task Baseline Agreement SDM-92-097

Developed by: Scientific Computing Unit, INEL

Funded by: Transuranic Waste Programs Unit, INEL

1.2 Software Module

\$Workfile: wastedlg.h $\$$

\$Revision: 2.0 \$ 
// The waste dialog provides the user interface to identify the waste

// container type, the matrix type, and the net weight of the container

$/ /$ being processed.

$1 /$

// 3. Interface Description

$1 /$

$1 /$

// 4. History

$1 /$

// Version 2.0 Release, April, 1996 (L. V. East)

// User interface improvements, additional data integrity tests and additional

// calculational capabilities as outlined in INEL/RWMC System Change Request

// No. 13623 (February, 1996). See Software Requirements specification,

// INEL-96/0056, and Software Design Description, INEL-96/0057, for a detailed

// description of this program.

$1 /$

// Rev 1.0 05 Oct $199315: 25: 36$ esm

// Initial release.

$1 /$

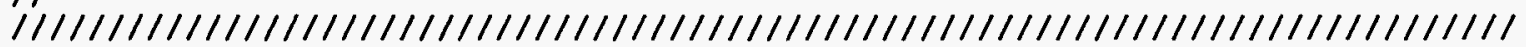

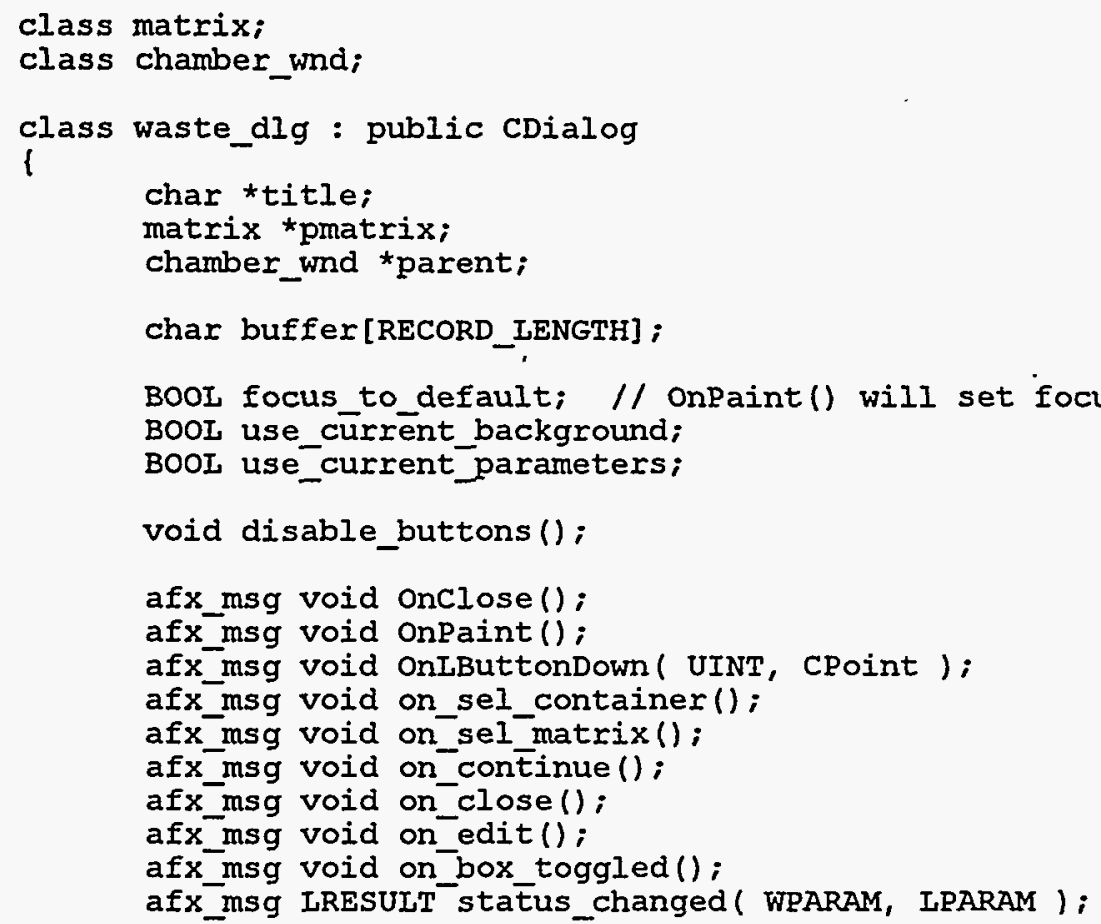

\#endif 


\section{Appendix B}

\section{Data Files}




\section{Appendix B}

\section{Data Files}

Listings of he following SAS data files are contained in this Appendix:

ACTCRATE.DAC

ACTCRATE.CAC ${ }^{(1)}$

AS.INI

ASSAYSYS.INI (2)

CAC.INI ${ }^{(1)}$

CALIB.DAC

CALIB.CAC ${ }^{(1)}$

CHISQ.DAC

CHISQ.CAC ${ }^{(1)}$

CONTAIN.LST

CORREL.DAC

CORREL.CAC ${ }^{(1)}$

DAC.INI

DEFAULT.GAM

MATRIX.LST

NUCLIDE.DAT

OPTIONS.DAC

OPTIONS.CAC ${ }^{(1)}$

PASCRATE.DAC

PASCRATE.CAC ${ }^{(1)}$

REGRESS.DAC ${ }^{(3)}$

REGRESS.CAC ${ }^{(1,3)}$

SAMPLE.001 ${ }^{(4)}$

(1) This file is associated with the Crate Assay Chamber which is not currently in use.

(2) This file is updated each time the program exits; the listing in this appendix is an example only.

(3) This file is not currently used.

${ }^{(4)}$ Sample "save" file; included as an example only. 


\section{B.1 ACTCRATE.DAC}

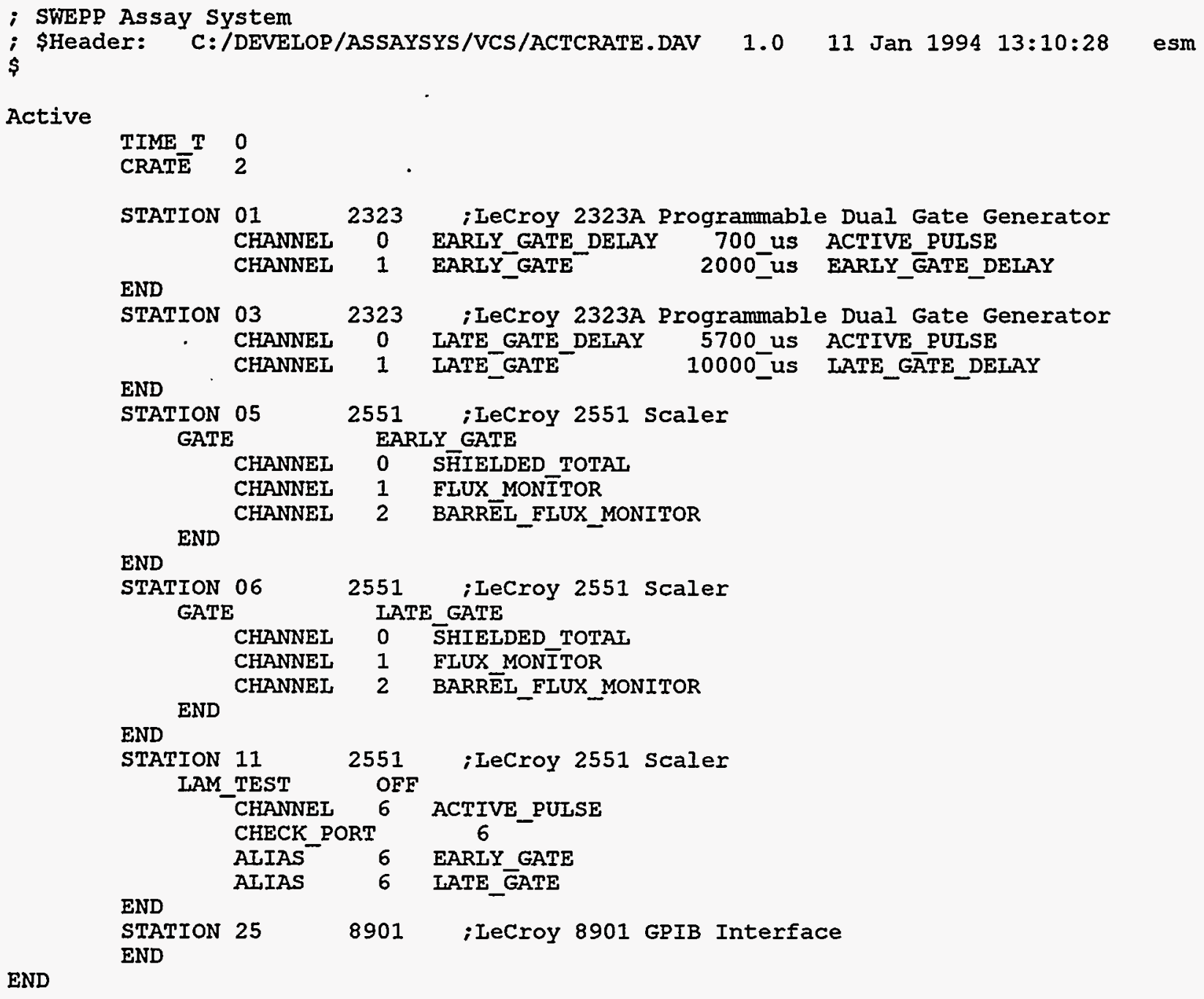

END

\section{B2. ACTCRATE.CAC}

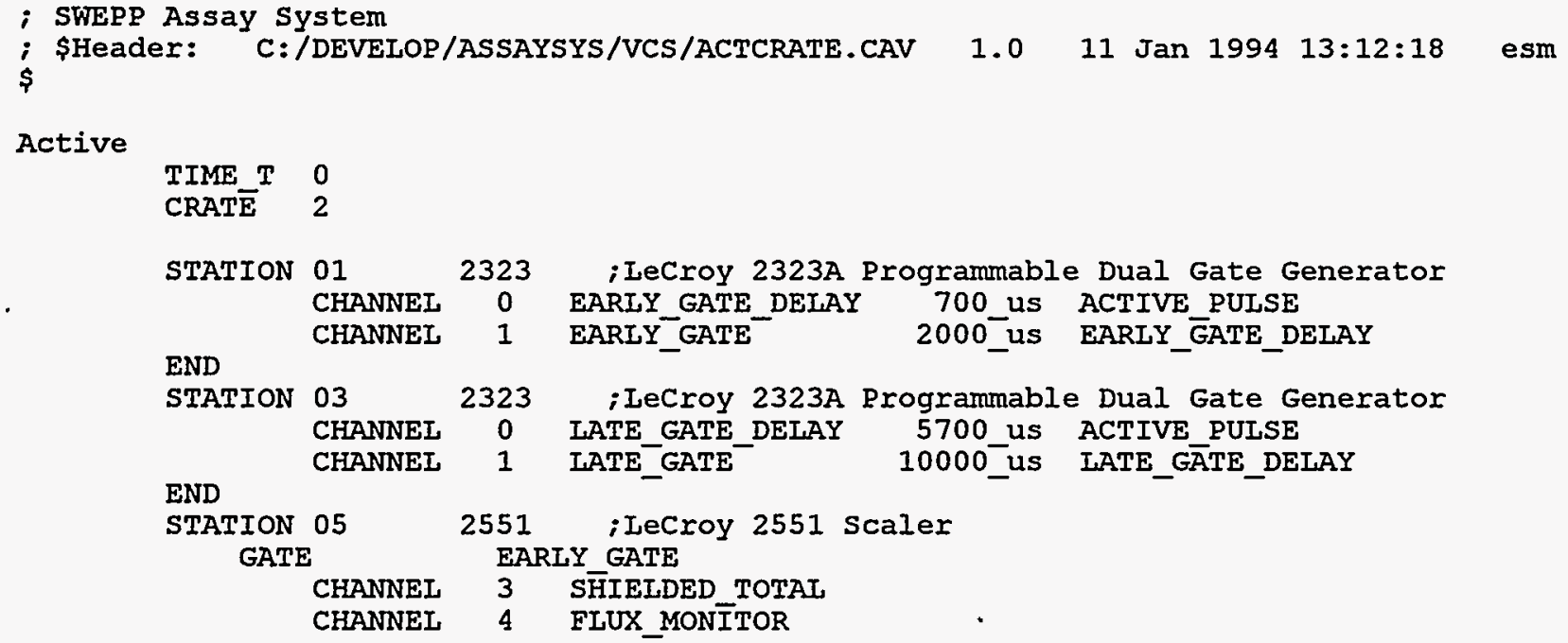




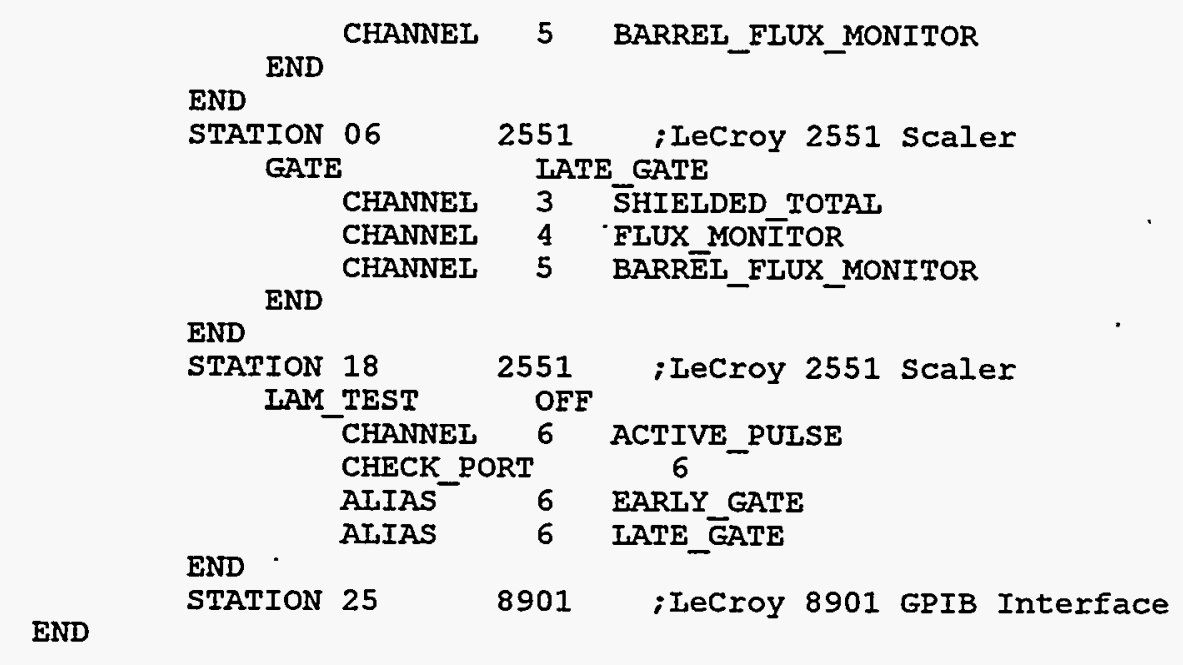

\section{B3. AS.INI}

; SWEPP Assay System Version 2.0

; Modified 1 Apr 1996 for Version 2.0 Release

; Customization parameters, run time files and directories

; for the SWEPP Passive/Active Neutron Assay System

; $\star \star$ NOTE: Entries are case sensitive! $* \star$

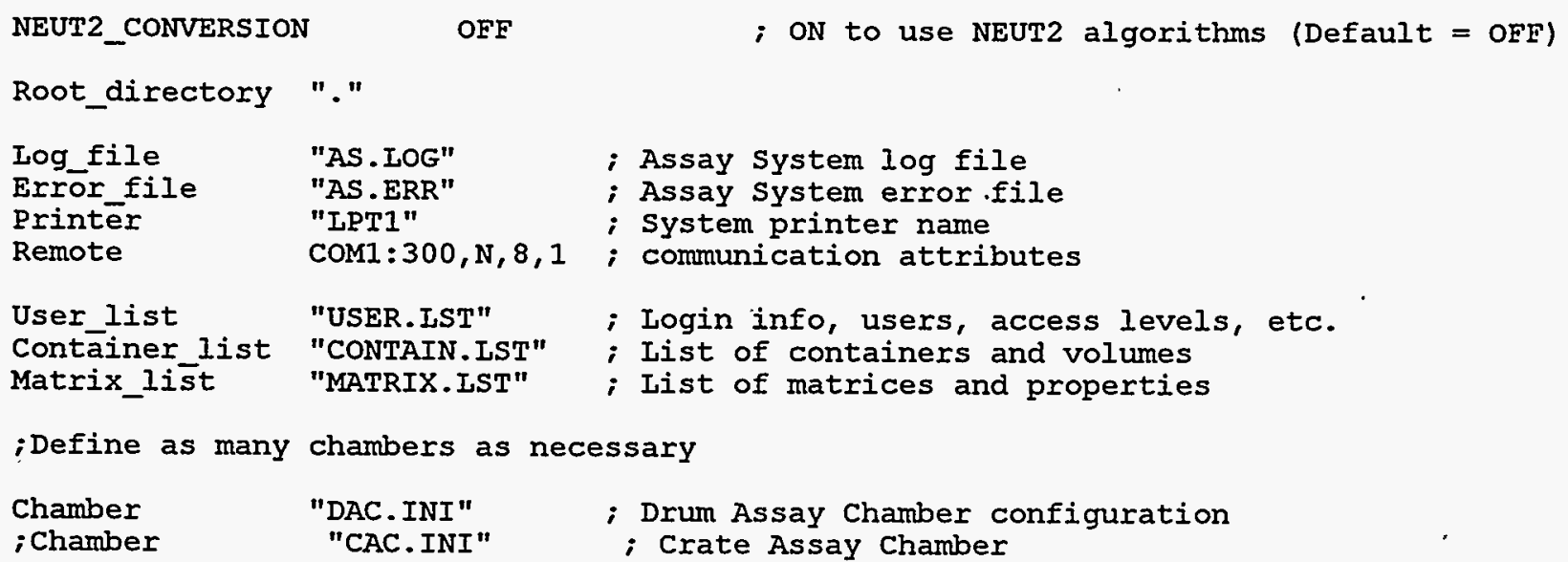

\section{B4. ASSAYSYS.INI}

[Drum Assay Chamber]

rect $=0000 \quad 0000 \quad 06870436$

$\max =0$

$i \mathrm{con}=0$

color $=16777088$

[Main Window]

réct $=0063 \quad 00290758 \quad 0530$

$\max =0$

acq $=0232 \quad 0282$

dms $=0224 \quad 0297$

$\mathrm{msg}=0293 \quad 0397$

[Crate Assay Chamber]

rect $=0021 \quad 00410707 \quad 0451$ 


\section{B5. CAC.INI}

; SWEPP Assay System Version 2.0

; Modified 1 Apr 1996 for Version 2.0 release

Chamber_name

"Crate Assay Chamber"

$\begin{array}{ll}\text { : Menu options } & \\ \text { SUMMARY_REPORT } & \text { ON } \\ \text { BACKGROUND_CORRECTION } & \text { ON } \\ \text { AUTO_SAVE } & \text { ON }\end{array}$

; Customization parameters

; Passive background check parameters.

; Nominal Pu mass fractions (value followed by standard deviation)

i Directory and File locations DIRECTORIES

\begin{tabular}{|c|c|c|}
\hline $\begin{array}{l}\text { Root directory } \\
\text { Gamma-directory } \\
\text { Archive_directory } \\
\text { Background directory } \\
\text { Verification directory } \\
\text { Production directory }\end{array}$ & $\begin{array}{l}\text { "CAC" } \\
\text { "E: \" } \\
\text { "B: \CAC" } \\
\text { "BACKG" } \\
\text { "VERIF" } \\
\text { "PROD" }\end{array}$ & $\begin{array}{l}\text {; chamber's root directory } \\
\text {; directory for gamma data } \\
\text {; chamber's archive directory }\end{array}$ \\
\hline
\end{tabular}

END

FILES

END

\begin{tabular}{|c|c|c|}
\hline lt_mass_data & $\begin{array}{l}\text { "NUCLIDE.DAT" } \\
\text { "DEFAULT.GAM" }\end{array}$ & $\begin{array}{l}\text {; Nuclide properties } \\
\text {; Default mass fractions from gamma }\end{array}$ \\
\hline $\begin{array}{l}\text { ctory for the ch } \\
\text { s_options } \\
\text { ound_data } \\
\text { cation_data } \\
\text { e CAMAC_config } \\
\text { - CAMAC_config } \\
\text { ation } \\
\text { ation }\end{array}$ & $\begin{array}{l}\text { "OPTIONS.CAC" } \\
\text { "BACKGRND" } \\
\text { "OOO496" } \\
\text { "PASCRATE.CAC" } \\
\text { "ACTCRATE.CAC" } \\
\text { "CAIIB.CAC" } \\
\text { "CORREL.CAC" } \\
\text { "CHISQ.CAC" }\end{array}$ & $\begin{array}{l}\text {; last background data } \\
\text {; last verification data } \\
\text {; passive CAMAC crate configuration } \\
\text {; active cAMAC crate configuration } \\
\text {; chamber calibration coefficients } \\
\text {; equation correlation coefficients } \\
\text {; Chi-Square parameter file }\end{array}$ \\
\hline
\end{tabular}

\section{B6. CALIB.DAC}

CHAMBER

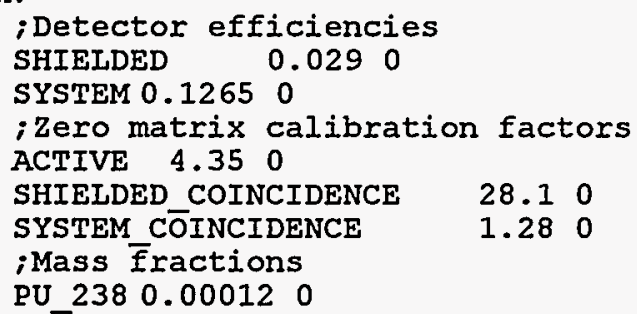


INEL-96/0057

END

$$
\begin{aligned}
& \text { PU_239 } 0.938260 \\
& \text { PU_240 } 0.05820 \\
& \text { PU_241 } 0.00340 \\
& \text { PU_242 } 0.000240
\end{aligned}
$$

\section{B7. CALIB.CAC}

; SWEPp Assay system

; \$Header: C:/DEVELOP/ASSAYSYS/VCS/CALIB.CAV $\quad 1.0 \quad 11$ Jan 1994 13:12:18 esm \$ CHAMBER

$$
\begin{aligned}
& \text {; Detector efficiencies } \\
& \text { SHIELDED } \quad 0.0290 .0 \\
& \text {; Zero matrix calibration factors } \\
& \text { SHIELDED COINCIDENCE } 84.0 \quad 0.0 \\
& \text {; Pu mass fractions } \\
& \begin{array}{lll}
\text { PU } 238 & 0.00012 & 0.0
\end{array} \\
& \begin{array}{lll}
\text { PU_239 } 0.93826 & 0.0
\end{array} \\
& \begin{array}{lll}
\mathrm{PU} 240 & 0.0582 & 0.0
\end{array} \\
& \begin{array}{lll}
\mathrm{PU} 241 & 0.0034 & 0.0
\end{array}
\end{aligned}
$$$$
\text { SYSTEM } \quad 0.1265 \quad 0.0
$$$$
\text { ACTIVE } 18.5 \quad 0.0
$$$$
\text { SYSTEM_CŌINCIDENCE } 2.92 \quad 0.0
$$

END

PU_242 0.000240 .0

\section{B8. CHISQ.DAC}

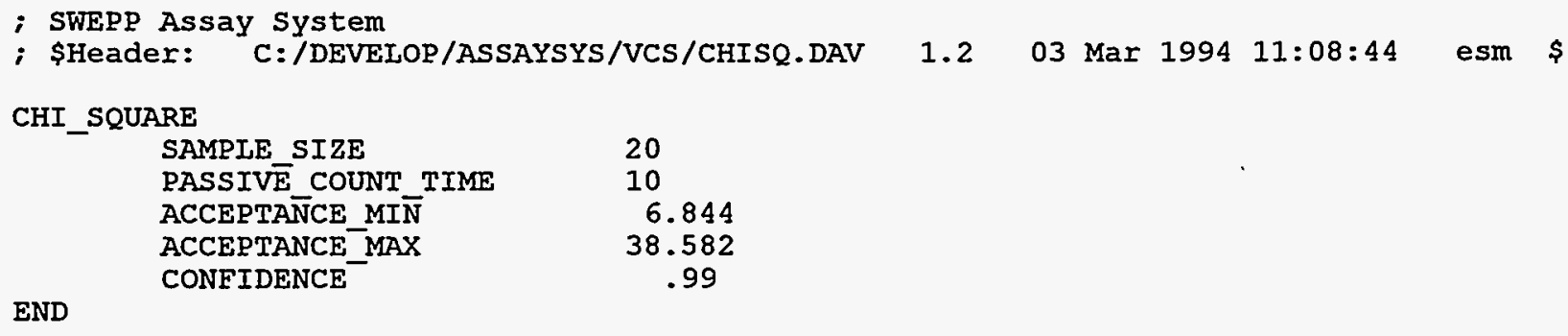

END

\section{B9. CHISQ.CAC}

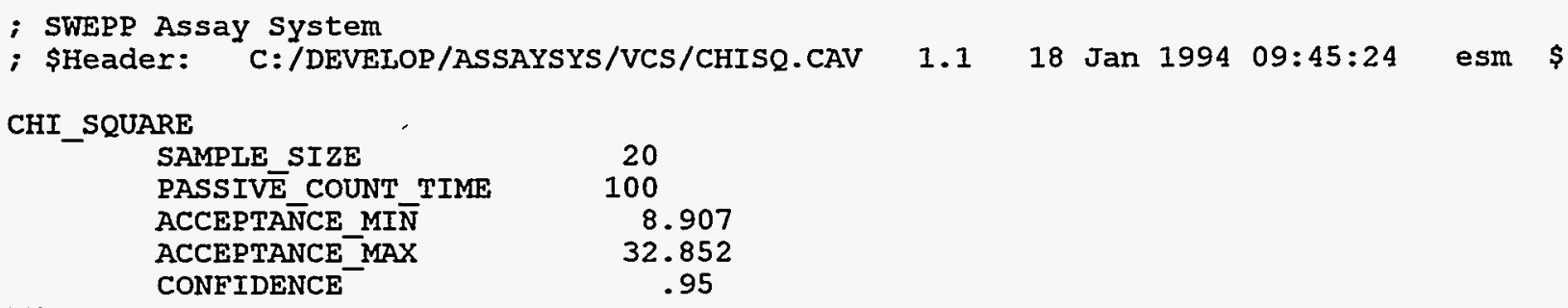

END

\section{B10. CONTAIN.LST}

CONTAINER

TYPE 55 gallon drum

VOLUME 208

END 
INEL-96/0057

\section{B11. CORREL.DAC}

; SWEPP Assay system

; \$Header: C:/DEVEIOP/ASSAYSYS/VCS/CORREL.DAV $1.0 \quad 11$ Jan 1994 13:10:42 esm

$\$$

; Correlation coefficients from the NEUT2 FORTRAN program and IA-10774-MS,

; "The Los Alamos Second-Generation System for Passive and Active

; Neutron Assays of Drum-Size Containers", by J.T. Caldwell, et al.,

; September 1986

$\begin{array}{cc}\text { ABSORBER INDEX_CORRELATION } \\ \text { AI_DEFAULT } & 1.0 \\ \text { AI_MIN } & 1.0\end{array}$

END

MODERATOR INDEX CORREIATION

; IA-10774-MS, Equation (3), Page 13

AO 1.0

A1 0.226

A2 1.04

A3 0.2924

MI MIN 0.0

END

MI_MAX 0.75

ACTIVE_MATRIX_CORRECTION_EACTOR

; factor correlated to absorption index

; Equations (8) and (9), Page 23

AI THRESHOID 2.72

$A M \bar{C}=A I \_C O \quad 0.5404$

AMCE_AI_C1 0.612

- factor correlated to moderator index

; Equations (10) and (11), Page 24

MI_THRESHOLD 0.4

AMC $\overline{\mathrm{C}}$ MI CO $\quad 0.483$

END

AMCE_MI_C1 $\quad 1.817$

ACTIVE_MASS

; interrogation background correlation

; Equation (17), Page 35

AI MI CO 0.00359

AI_MI_C1 0.00139

- $\quad$ background scale factor

; value from NEUT2

ACTIVE_BACKGROUND_SCAIE_FACTOR 0.977

; coefficients for active mass error calculation

; value from NEUT2

ACTIVE MASS ERR CO 0.003

; Equation (22), Page 41

ACTIVE MASS ERR C1 0.05

END

ACTIVE_MASS_ERR_C2 0.05

PASSIVE MASS

; system coincidence correction factor correlation coefficients

; Equation (14), Page 26

SYSTEM COINCIDENCE CF CO

SYSTEM COINCIDENCE CE-C1 0.4187

0.5967

; systēm coincidence correction factor correlation coefficients

; Equation (14), Page 26

SHIELDED_COINCIDENCE_CF_CO 0.8092

SHIELDED_COINCIDENCE_CF_C1 0.2337 
END

PLUTONIUM_MASS_SELECTION_CRITERIA

END MĀSS_TFRESHOLD 10.0

AMERICIUM MASS

; Americium sludge mass computation

; coefficients from NEUT2

AM SLUDGE MASS CO 1.12

AM SIUDGE MASS C1 76.0

AM SLUDGE MASS C2 $2.93 \mathrm{e}-4$

END

AM SLUDGE MASS C3 0.30

\section{B12. CORREL.CAC}

; SWEPP Assay system

;Header: C:/DEVELOP/ASSAYSYS/VCS/CORREL.CAV $\quad 1.0 \quad 11$ Jan 1994 13:12:18, esm

; Correlation coefficients from the NEUT2 FORTRAN program and I.A-10774-MS,

; "The Los Alamos Second-Generation System for Passive and Active

; Neutron Assays of Drum-Size Containers", by J.T. Caldwell, et al.,

; September 1986

ABSORBER_INDEX_CORRELATION

AIIDEFAULT 0.18

END

$\begin{array}{ll}\text { AI_MIN } & 0.1\end{array}$

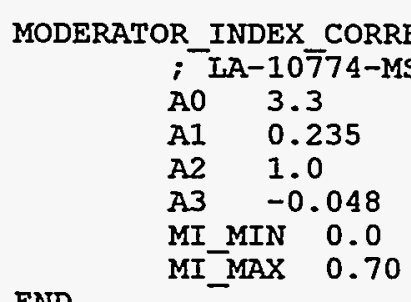

END

ACTIVE_MATRIX_CORRECTION FACTOR

; factor correlated to absorption index

i Equations (8) and (9), Page 23

AI THRESHOLD 2.72

$A M \bar{C}$ AI CO 0.5404

AMCF_AI_C1 0.612

- factor correlated to moderator index

; Equations (10) and (11), Page 24

MI_THRESHOLD 0.4

AMC $\bar{C}$ MI CO 0.483

END

AMCF_MI_C1 1.817

ACTIVE_MASS

; interrogation background correlation

; Equation (17), Page 35

AI MI CO 0.00359

AI_MI_C1 0.00139

; background scale factor

; value from NEUT2

ACTIVE_BACKGROUND_SCALE_FACTOR 1.0 


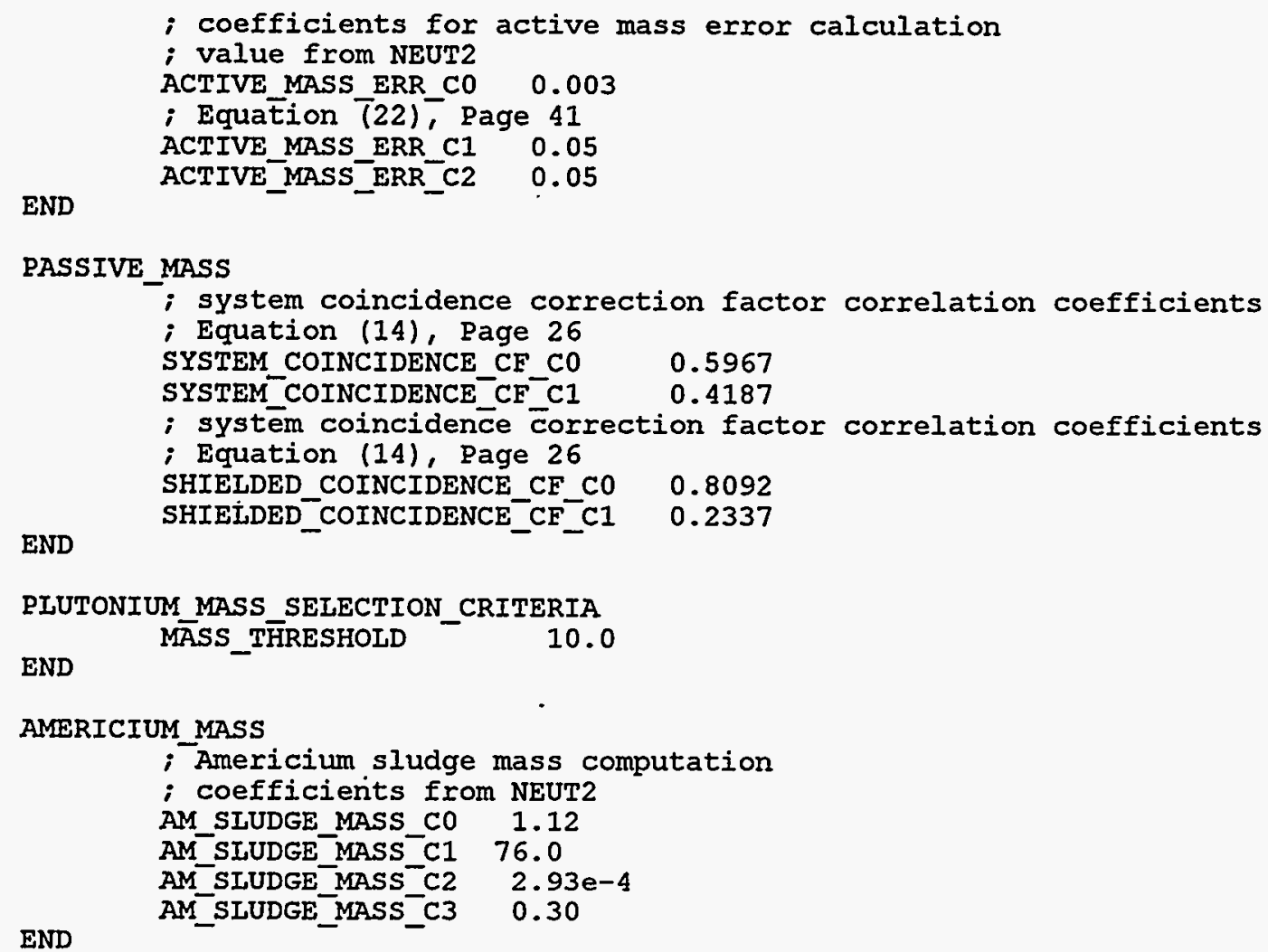

\section{B13. DAC.INI}

; SWEPP Assay System Version 2.0

; Modified 1 Apr 1996 for Version 2.0 release

; Assay chamber specific run time files and directories for the SWEPp Assay System. ; NOTE: Names are case sensitive!

Chamber_name

; Menu options SUMMARY REPORT AUTO SAVEE BACKḠROUND_CORRECTION

; Customization parameters ACTIVE FIRST

PASSIVE_DELAY

INCLUDE PU242

INCLUDE_U233

INCLUDE U235

USE_GAMMA PU DATA

MAX_SIDE_TAR_BASE
"Drum Assay Chamber"

$\begin{array}{ll}\text { ON } & ; \text { ON/OFF - Default }=\text { ON } \\ \text { ON } & ; \text { ON/OFF - Default }=\text { OFF } \\ \text { ON } & ; \text { ON/OFF - Default }=\text { ON } \\ & \\ \text { YES } & ; \text { YES/NO - Default = YES } \\ 15 & ; \text { Seconds delaY after active assay - } \\ \text { NO } & ; \text { DefauIt = } 10 \text { sec } \\ \text { NO } & ; \text { YES/NO - DefauIt = NO } \\ \text { YES } & ; \text { YES/NO - Default = NO } \\ \text { NO } & ; \text { YES/NO - Default = YES } \\ 0.20 & ; \text { Maximum fractional variation expected } \\ & ; \text { for side detector variation under good } \\ & ; \text { statistics conditions - Default = 0.15 }\end{array}$

- Passive background check parameters

NOMINAI_SG_BKGND $\quad 0.090$; Nominal shielded background coincidence rate.

NOMINAI_LG_BKGND $\quad 1.10$; Nominal system background coincidence rate.

MAXIMUM_BKGND_AGE $\quad 10.0$; Maximum time (hours) before new bkgnd run

required. 
; Nominal Pu mass fractions NOMINAL_PU240_FRACTION NOMINAI_PU241-ERACTION

(value followed by standard deviation)

; Directory and file names

DIRECTORIES

\begin{tabular}{|c|c|c|}
\hline $\begin{array}{l}\text { Root_directory } \\
\text { Gama_directory } \\
\text { Archive_directory } \\
\text { Background_directory } \\
\text { Verification directory } \\
\text { Production_directory }\end{array}$ & $\begin{array}{l}\text { "DAC" } \\
\text { "E:\" } \\
\text { "B:\DAC" } \\
\text { "BACKG" } \\
\text { "VERIF" } \\
\text { "PROD" }\end{array}$ & $\begin{array}{l}\text {; chamber's root directory } \\
\text {; directory for gamma data } \\
\text {; chamber's archive directory }\end{array}$ \\
\hline
\end{tabular}

END

0.05870 .010

0.00140 .010

FILES

; The nuclide data and mass fractions in the root directory for the system. Nuclide

Default_mass_data

"NUCLIDE.DAT" ; Nuclide properties

"DEFAULT.GAM" ; Default mass fractions from gamma

; The remaining files, specific to the assay chamber, are in the root

; directory for the chamber, a subdirectory of the system, specified above.

Process options

Background_data

Verification data

Passive CAMAC config

Active_CAMAC_config

; chamber options

"BACKGRND"

"000496"

- last background data

Calibrātion

"PASCRATE. DAC"

- last verification data

"ACTCRATE.DAC"

- passive CAMAC crate configuration

Correlation

END

Chi Square

"CORREL. DAC"

- active CAMAC crate configuration

; chamber calibration coefficients

- equation correlation coefficients

"CHISQ.DAC"

; Chi-Square parameter file

\section{B14. DEFAULT.GAM}

; SWEPP Assay system Version 2.0

; Modified 16 Apr 96 for Version 2.0 release.

PU MASS FRACTIONS

$\begin{array}{ccc}238 & 0.00015 & 0.00008 \\ 239 & 0.93940 & 0.00110 \\ 240 & 0.05870 & 0.00070 \\ 241 & 0.00140 & 0.00060 \\ 242 & 0.00025 & 0.00005\end{array}$

END

\section{B15. MATRIX.LST}

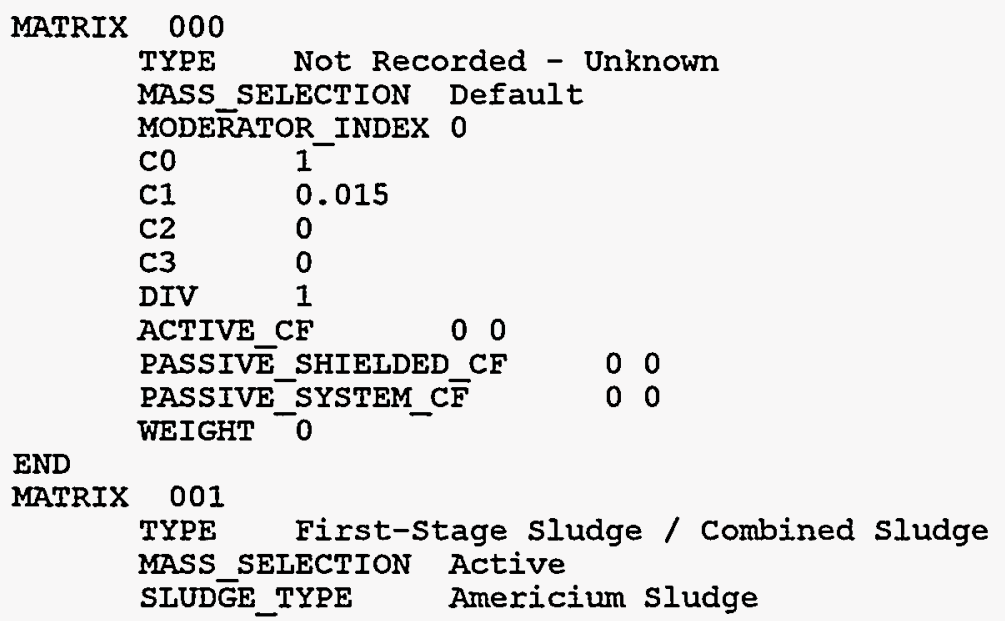




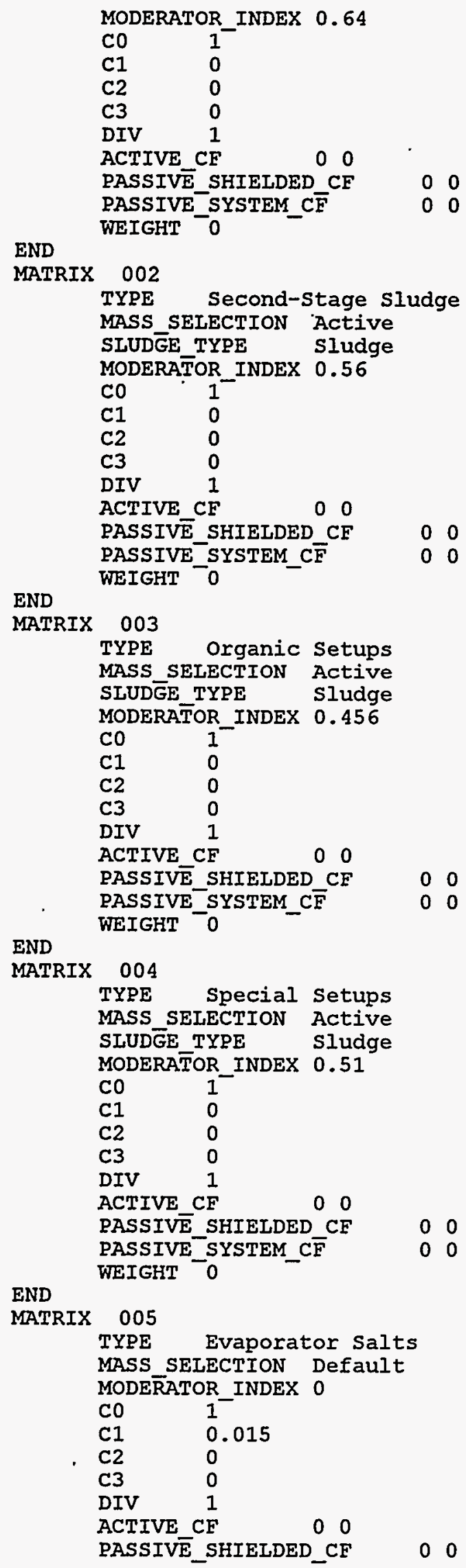




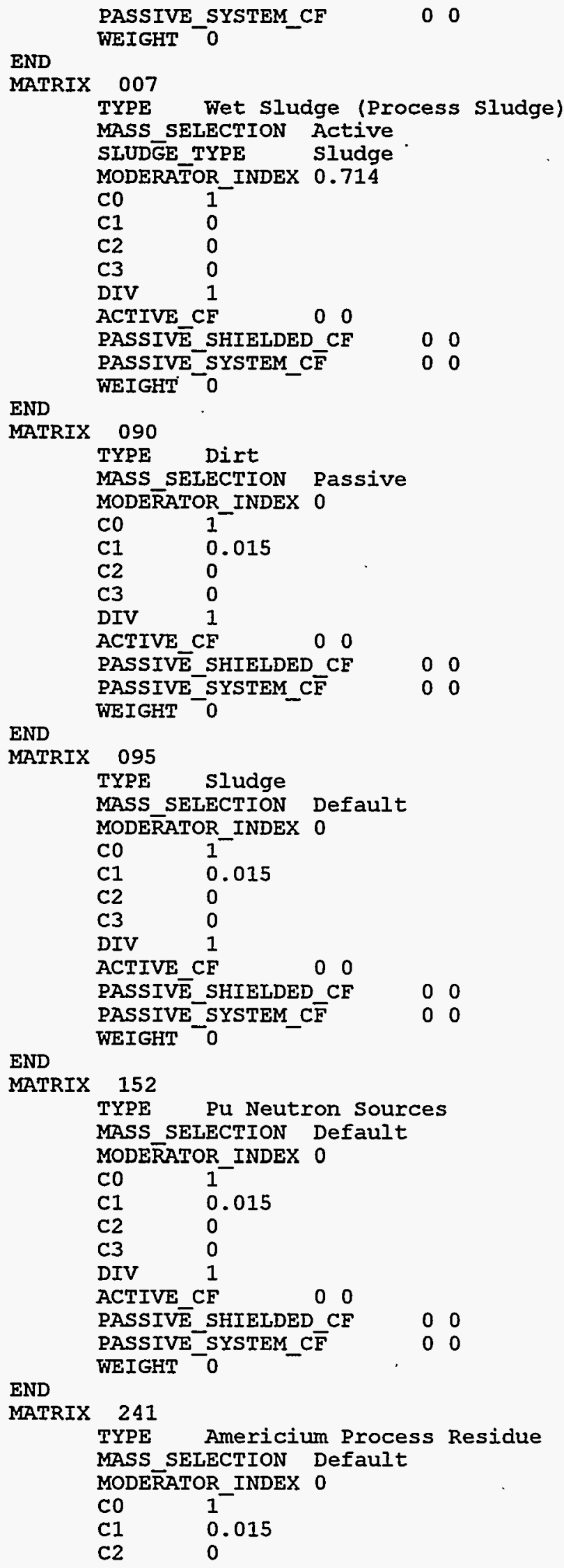




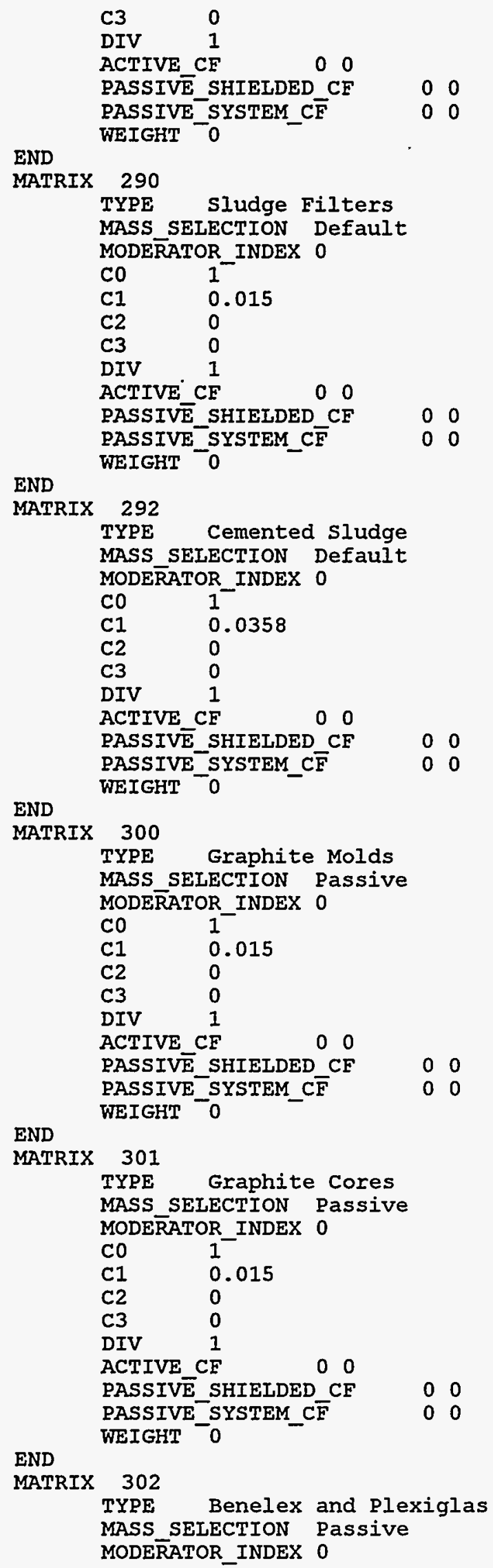


INEL-96/0057

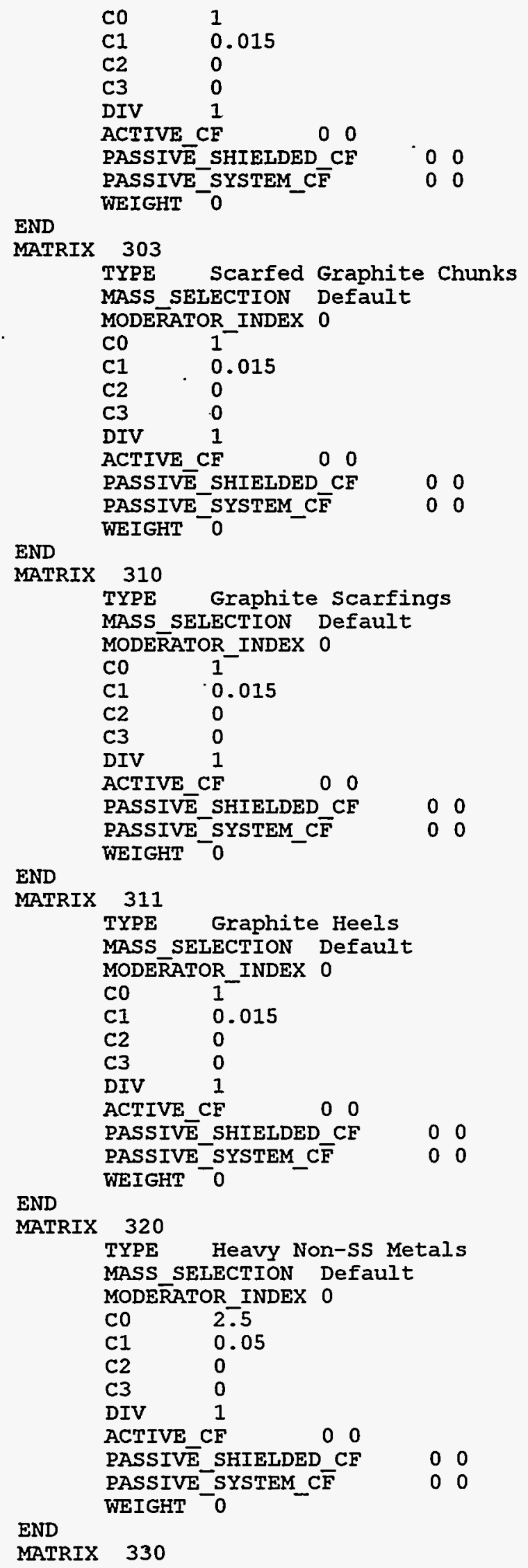

MATRIX 330 


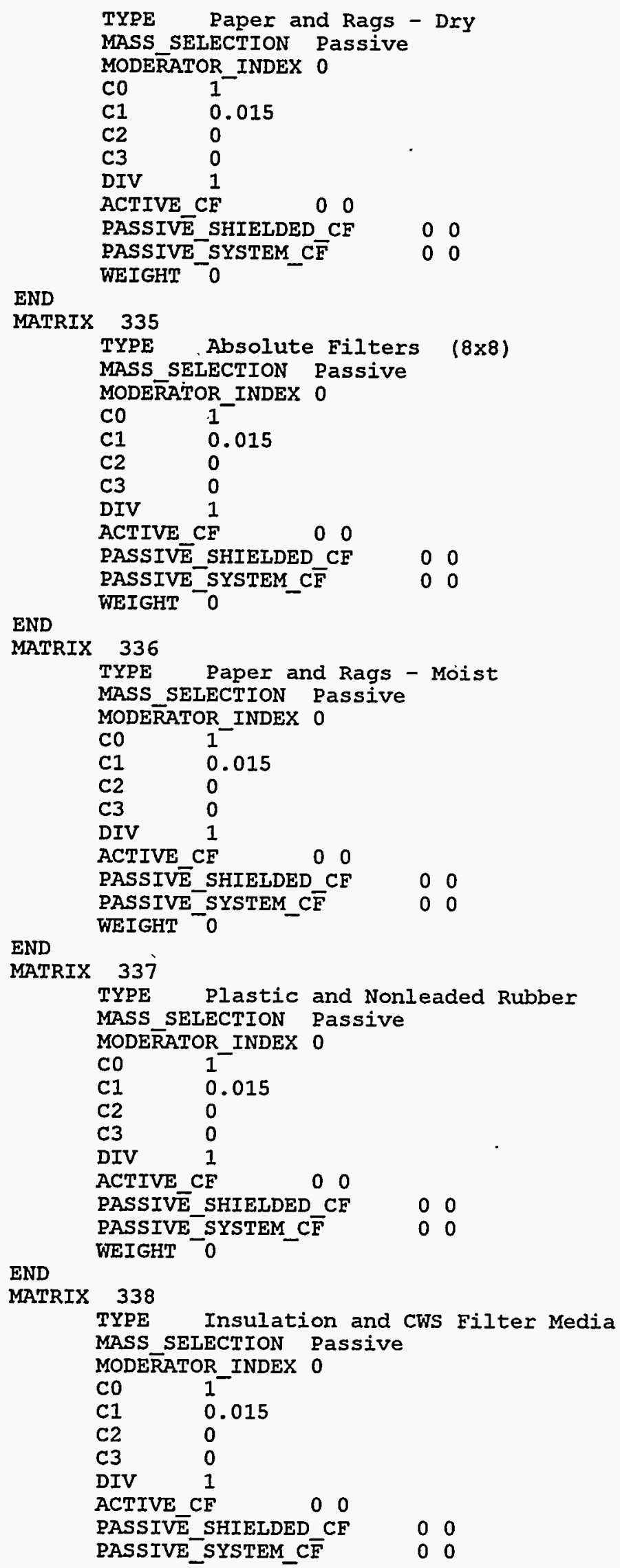


INEL-96/0057

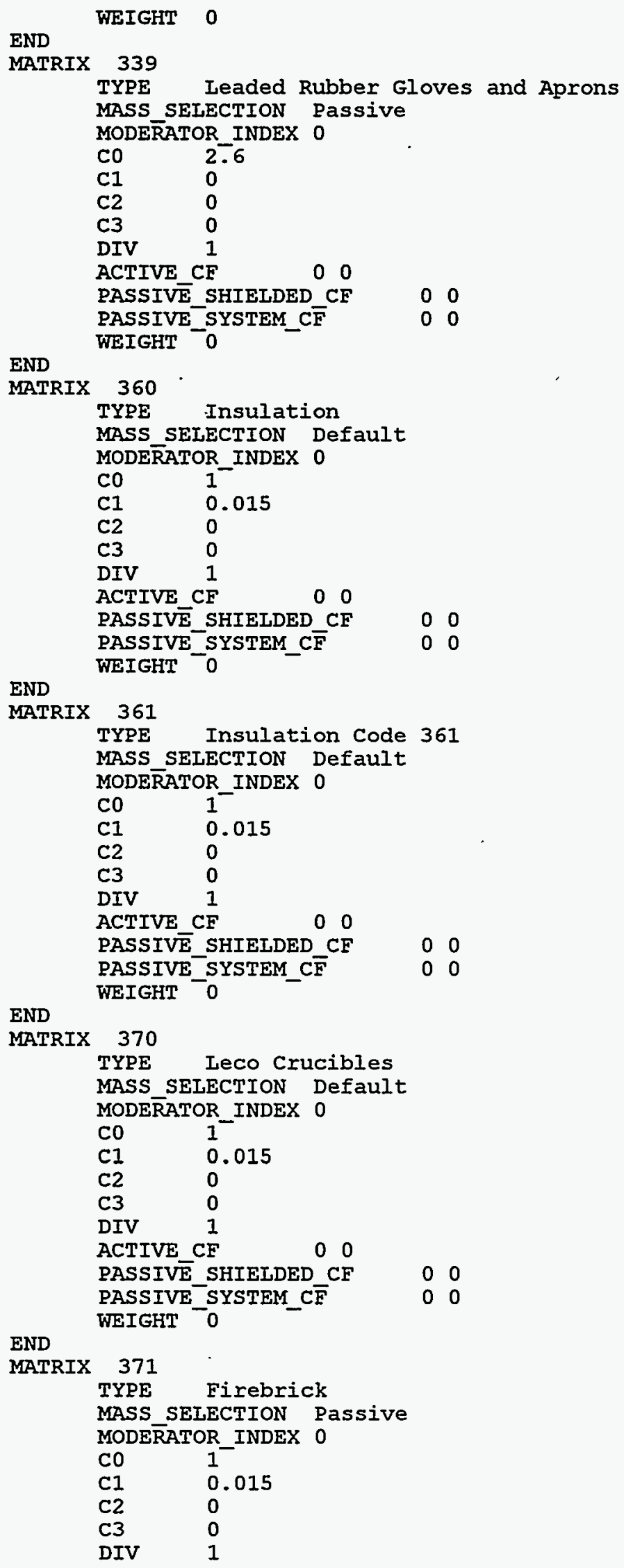




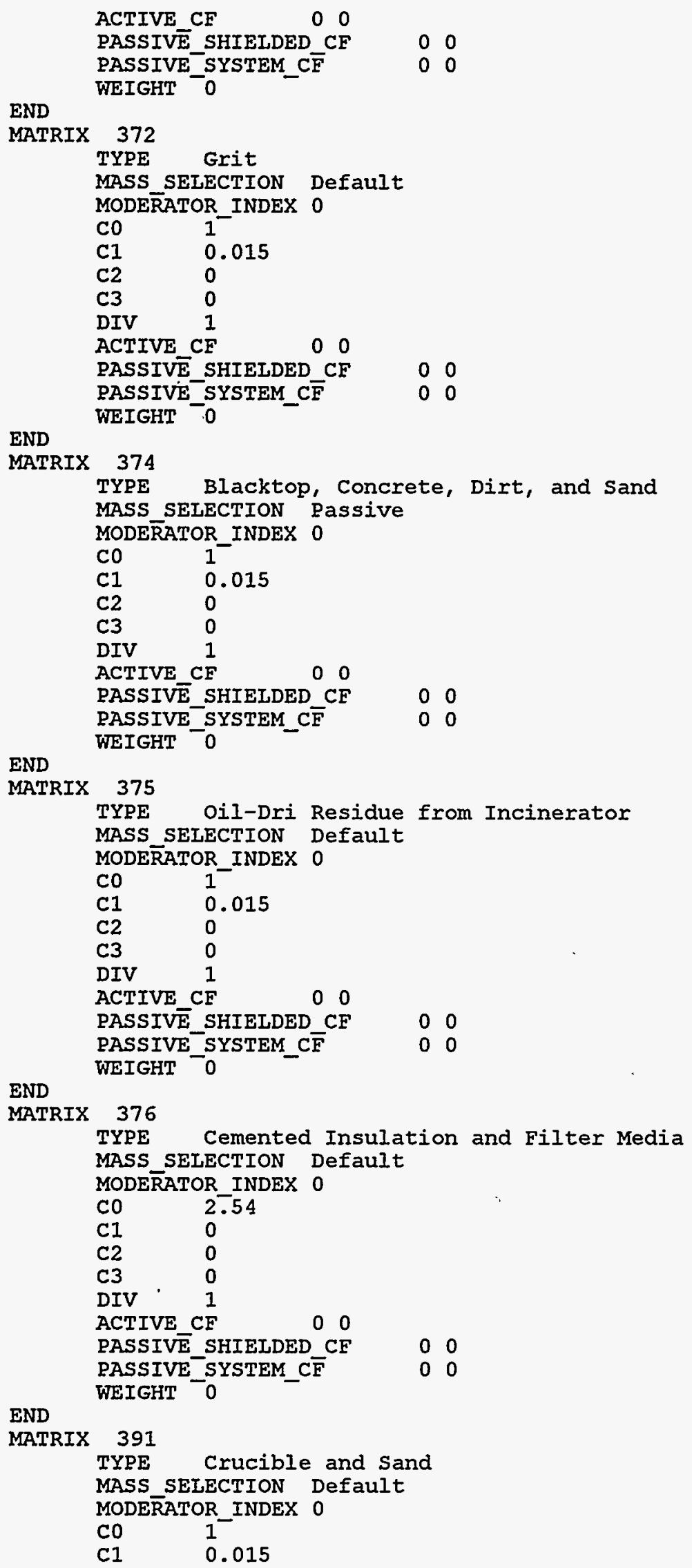


INEL-96/0057

END

$$
\begin{array}{llllll}
\text { C2 } & 0 & & & & \\
\text { C3 } & 0 & & & & \\
\text { DIV } & 1 & & & & \\
\text { ACTIVE_CF } & 0 & 0 & & & \\
\text { PASSIVE_SHIELDED_CF } & & 0 & 0 \\
\text { PASSIVE_SYSTEM_C } & & & 0 & 0 \\
\text { WEIGHT } & 0 & &
\end{array}
$$

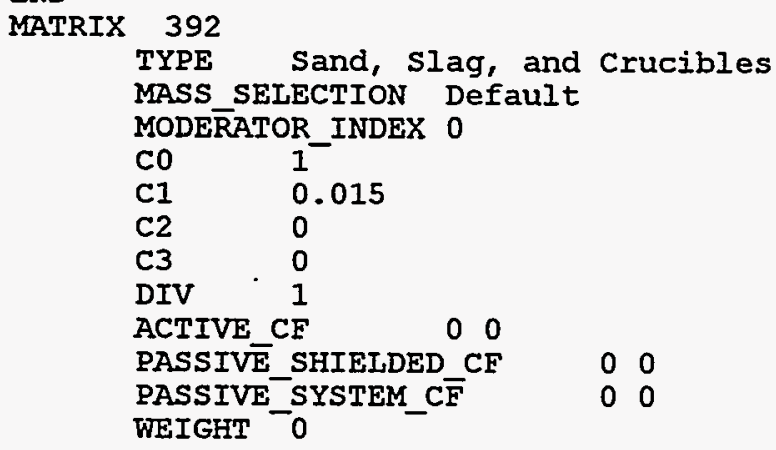




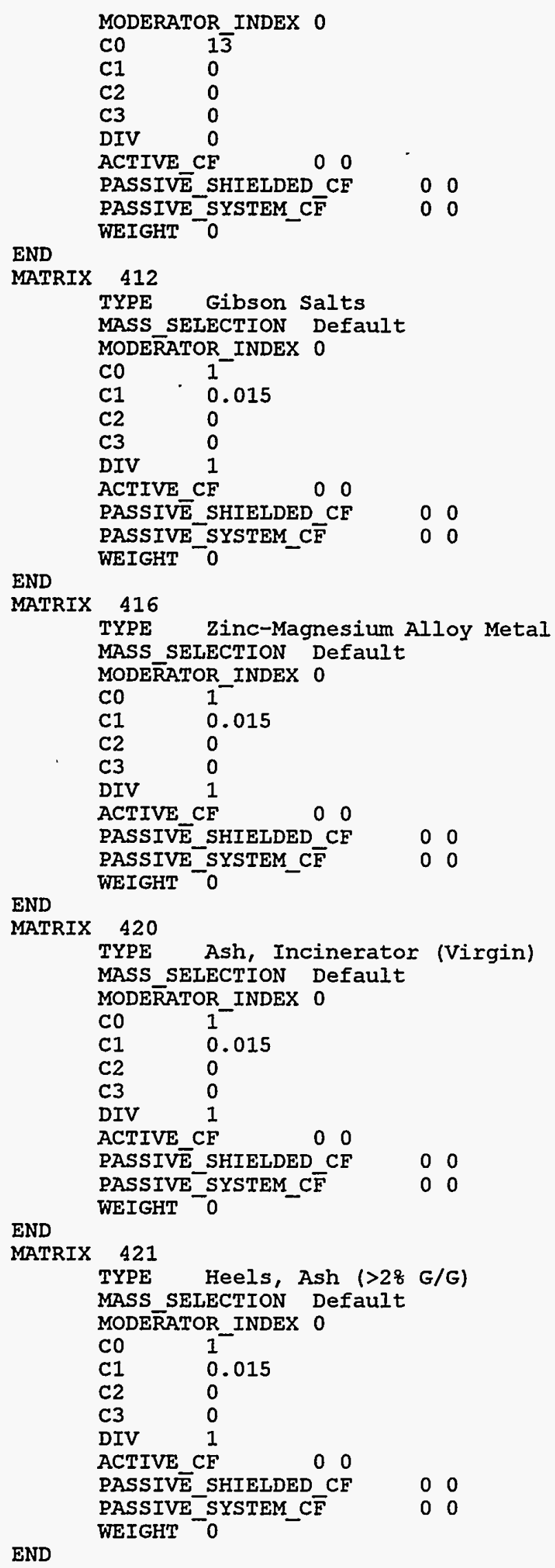




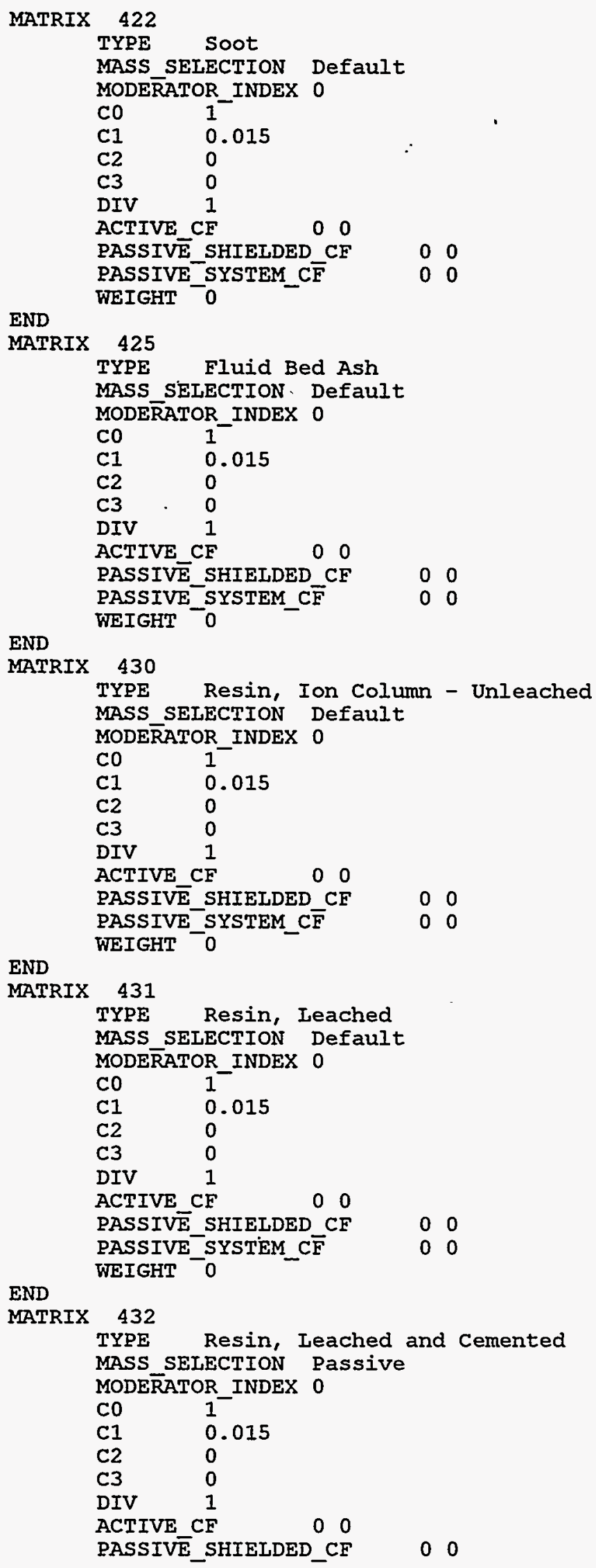




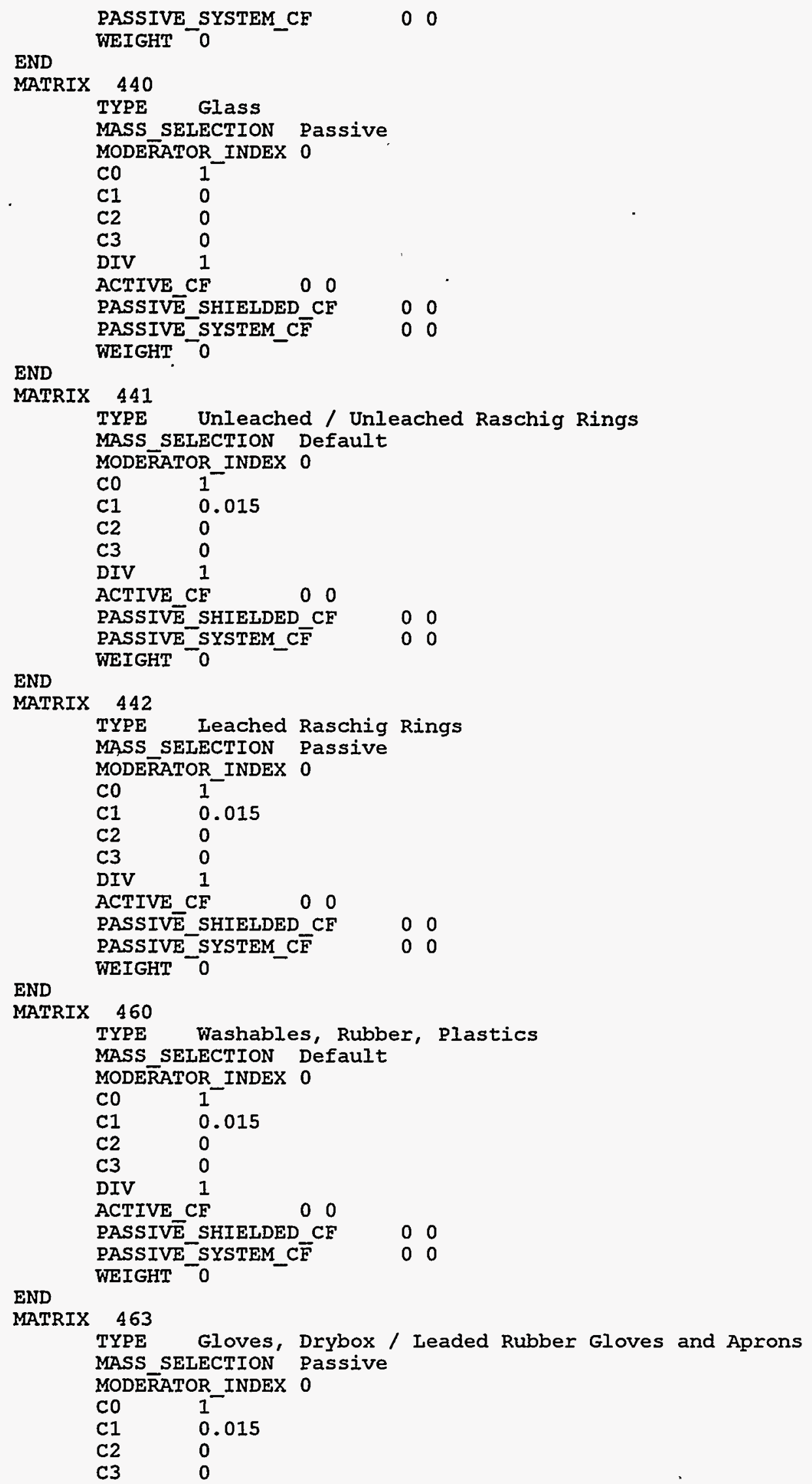


END
DIV
ACTIVE CF
00
PASSIVE SHIELDED CF
PASSIVE_SYSTEM_C $\overline{\mathrm{F}}$
00
00

WEIGHT 0

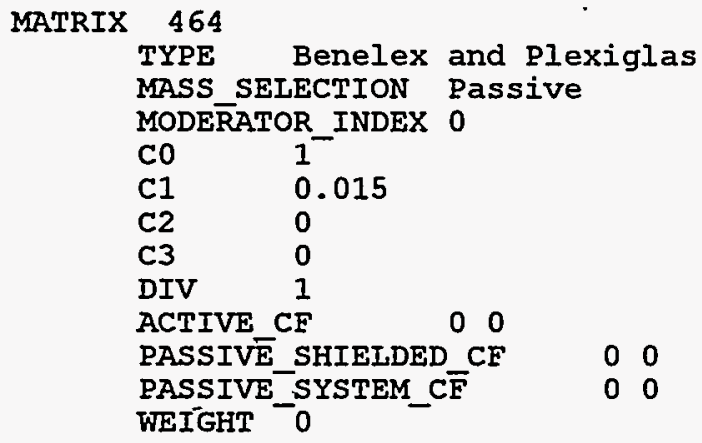




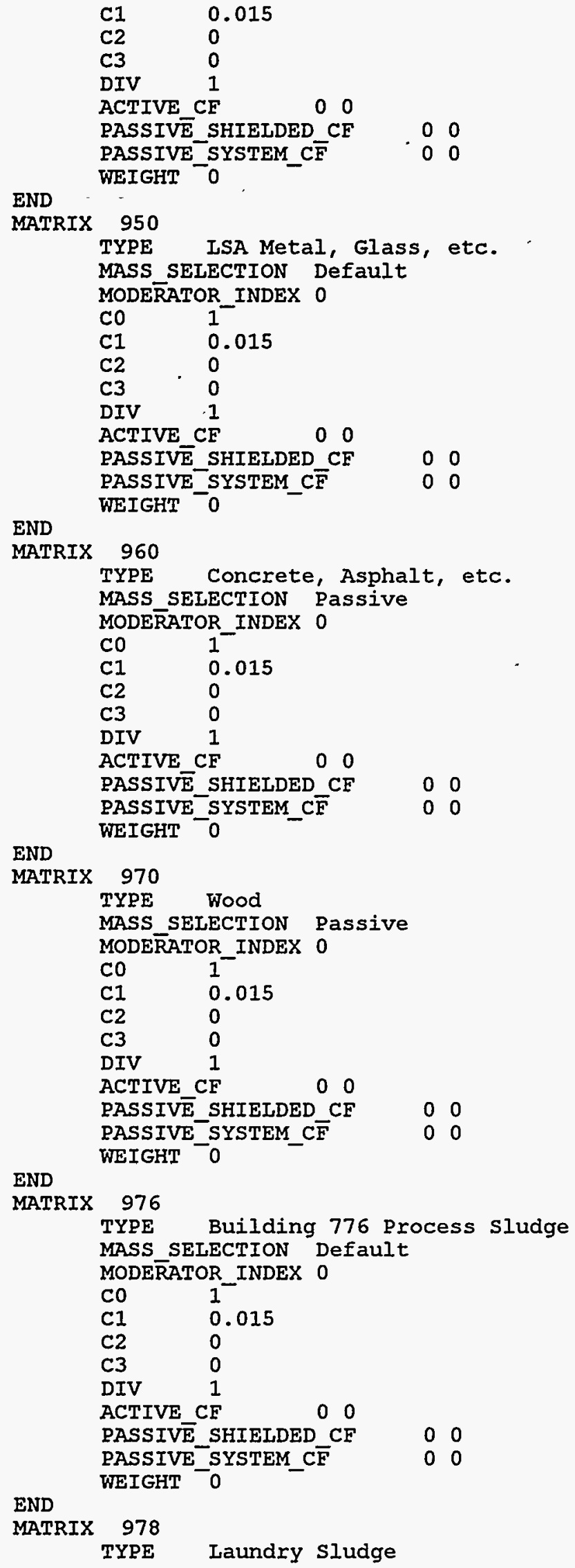

MATRIX 970

TYPE Wood

MASS SELECTION Passive

MODERATOR_INDEX 0

CO 1

C1 0.015

C2 0

C3 0

DIV 1

ACTIVE CF 00

PASSIVE_SHIELDED_CF $\quad 0 \quad 0$

PASSIVE_SYSTEM_C $\bar{F} \quad 00$

END

WEIGHT 0 


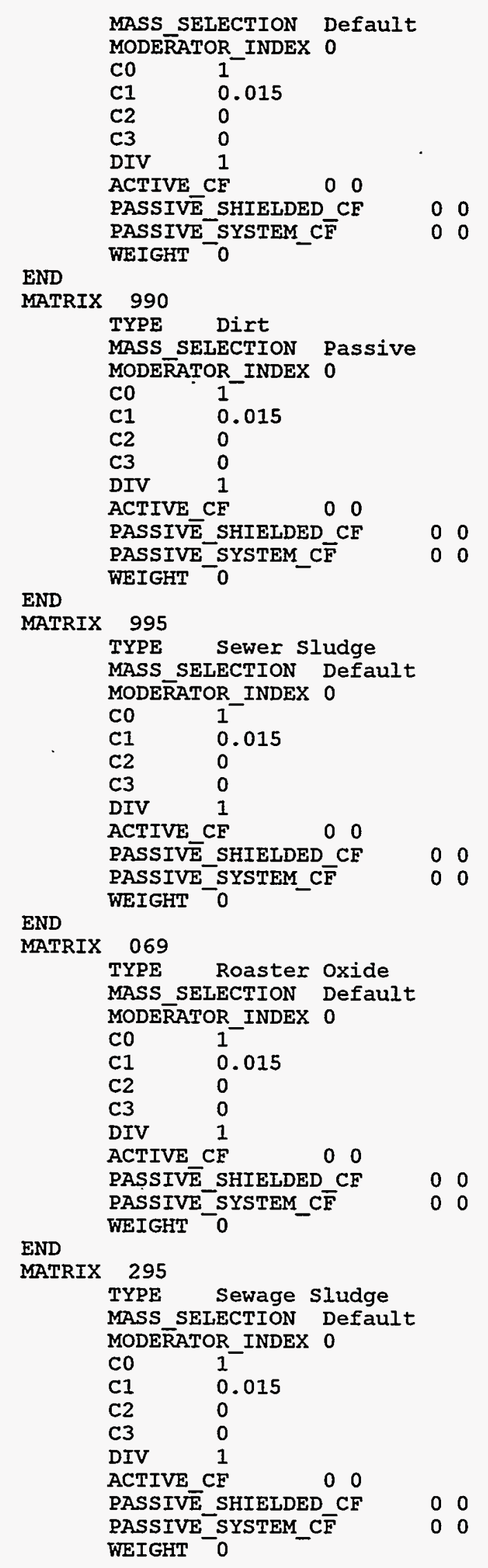




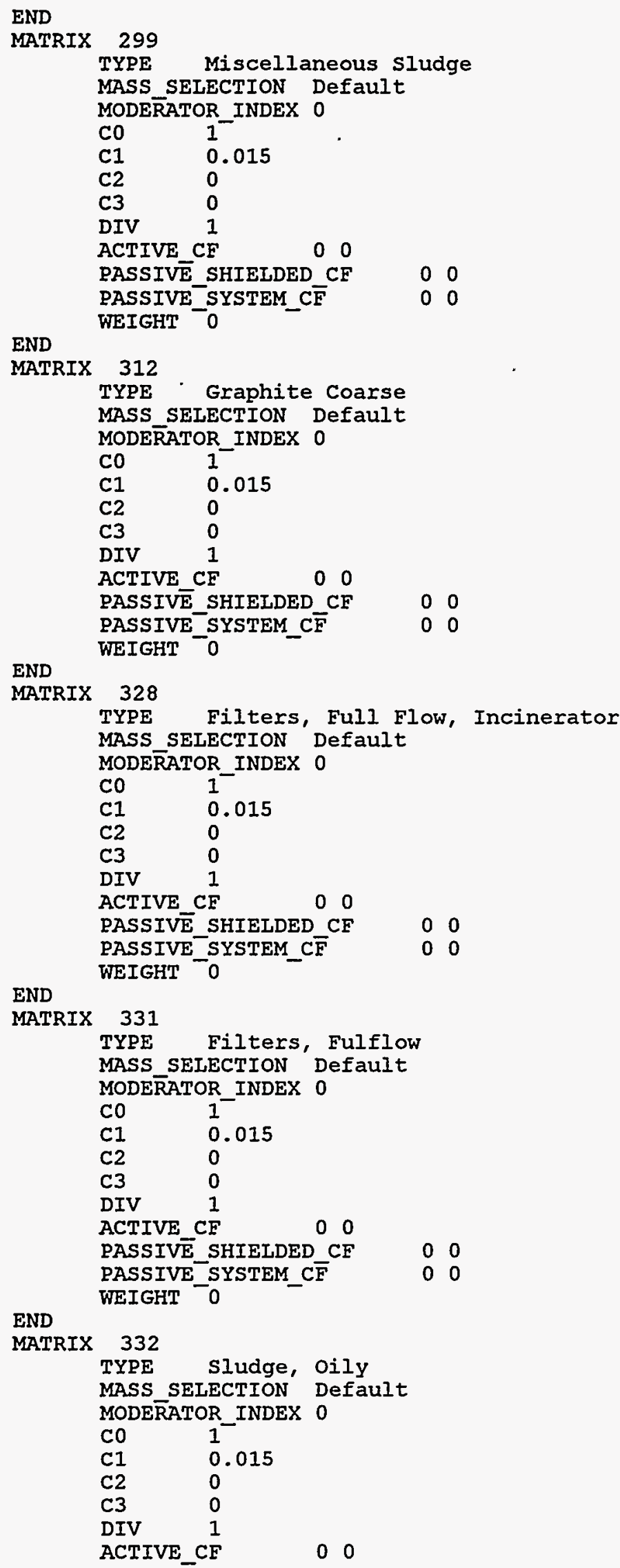


INEL-96/0057

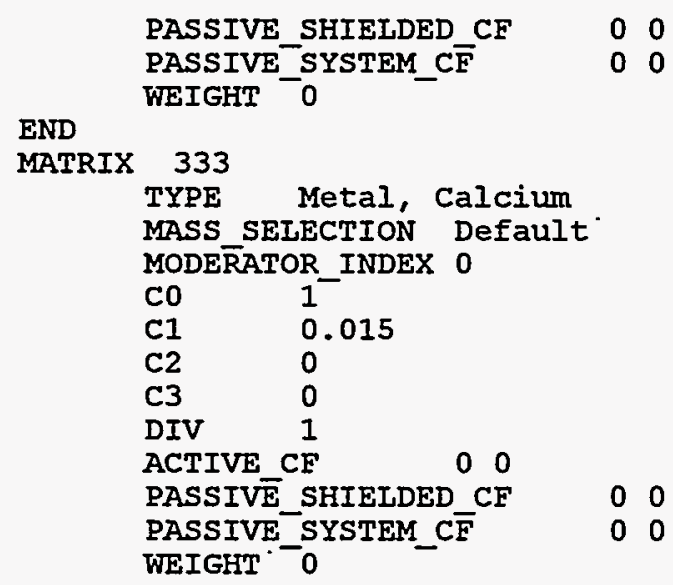




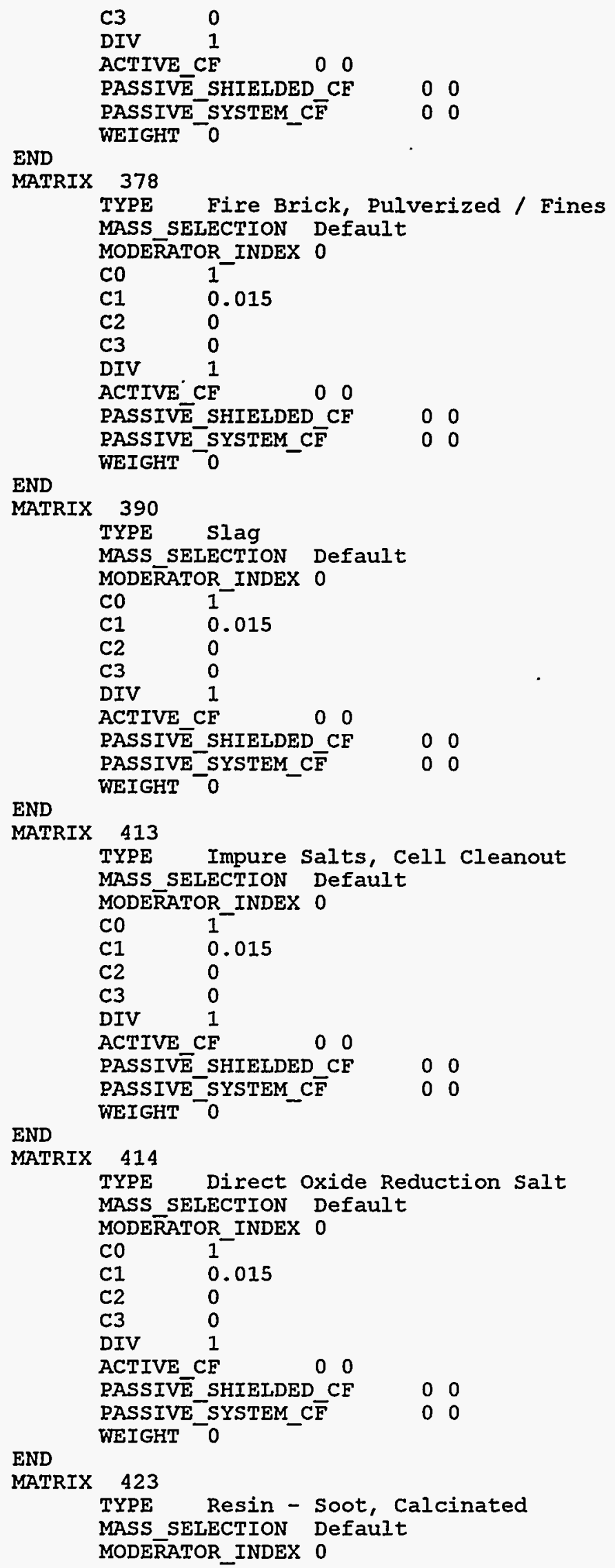




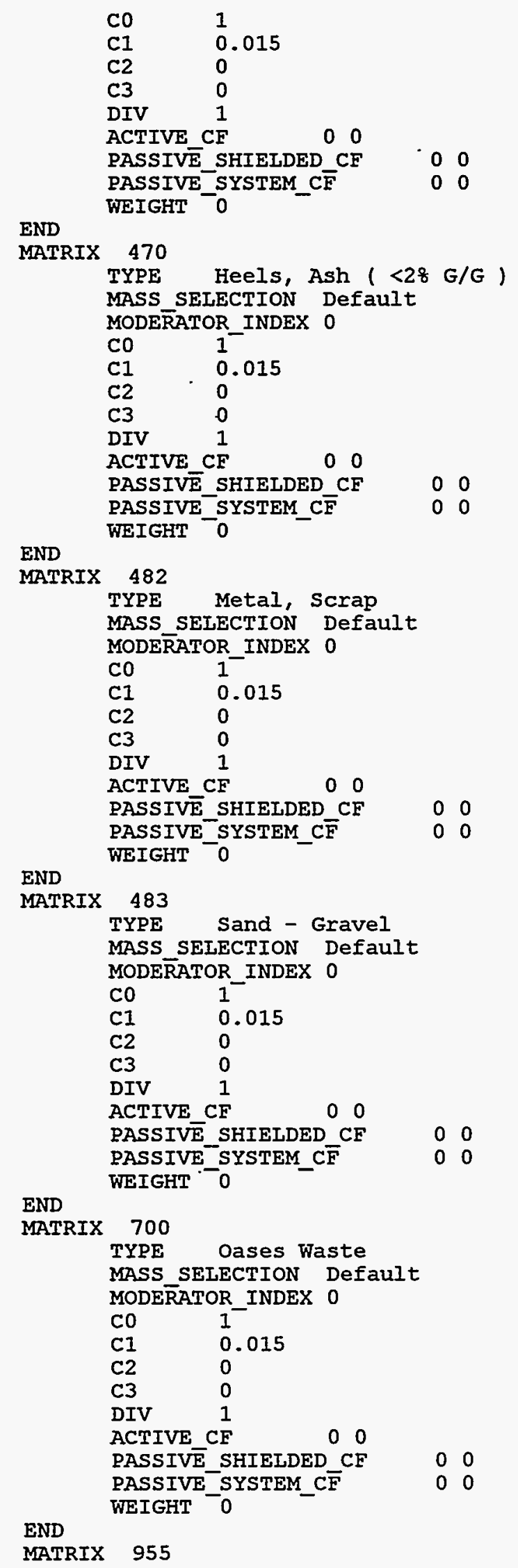




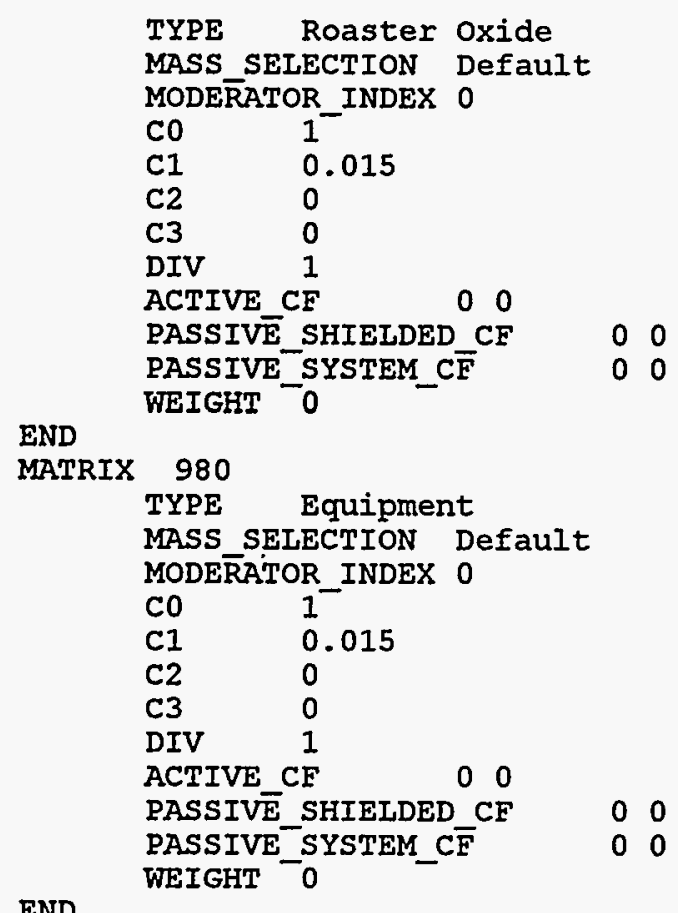

\section{B16. NUCLIDE.DAT}

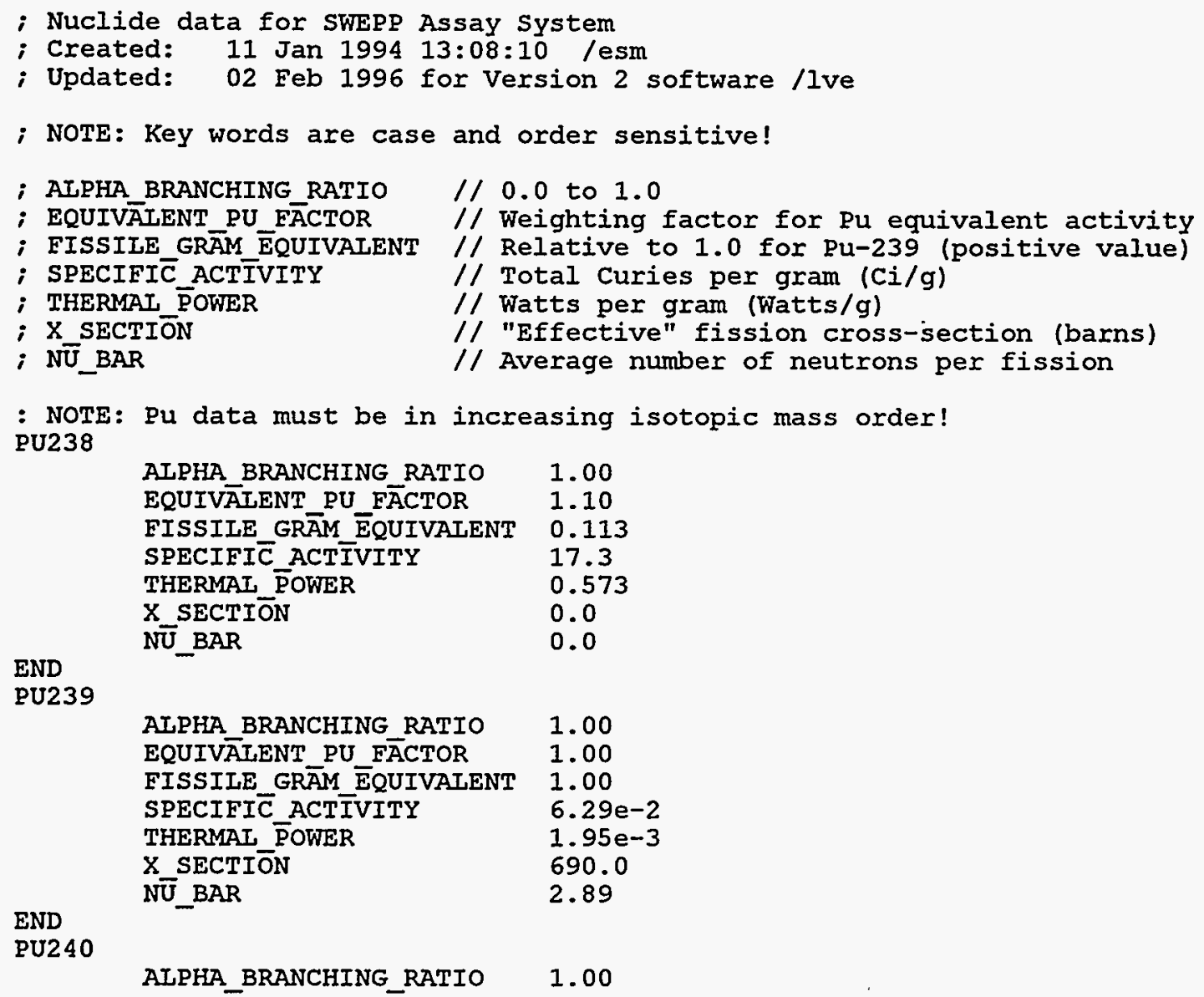


END

$\begin{array}{ll}\text { EQUIVALENT_PU FACTOR } & 1.00 \\ \text { FISSILE_GRAM EQUIVALENT } & 2.25 \mathrm{E}-2 \\ \text { SPECIFIC ACTIVITY } & 2.30 \mathrm{e}-1 \\ \text { THERMAI, POWER } & 7.16 \mathrm{e}-3 \\ \text { X_SECTION } & 0.0 \\ \text { NU_BAR } & 0.0\end{array}$

PU241

ALPHA BRANCHING RATIO

$2.45 e-5$ EQUIVALENT PU FACTOR

52.0

FISSILE_GRĀM ĒQUIVALENT 2.25

SPECIFIC ACTIVITY

$1.04 \mathrm{e} 2$

THERMAI $\overline{\text { POWER }}$

$3.31 e-3$

$X$ SECTION

0.0

END

NU $B A R$

0.0

PU242

$\begin{array}{ll}\text { ALPHA BRANCHING_RATIO } & 1.00 \\ \text { EQUIVAIENT_PU_FACTOR } & 1.10 \\ \text { FISSIIE_GRAM EQUIVAIENT } & 7.50 e-3 \\ \text { SPECIEIC ACTIVITY } & 3.97 e-3 \\ \text { THERMAL_ POWER } & 1.17 e-4 \\ \text { X_SECTION } & 0.0 \\ \text { NU_BAR } & 0.0\end{array}$

END

AM241

ALPHA BRANCHING RATIO 1.00 EQUIVĀIENT PU EĀCTOR 1.00

FISSIIE_GRAM EQUIVAIENT $1.87 \mathrm{e}-2$

SPECIFI $\bar{C}$ ACTIVITY $\quad 3.47$

THERMAL POWER $1.16 \mathrm{e}-1$

$X$ SECTION

0.0

$N \bar{U}$ BAR

0.0

END

U235

$\begin{array}{ll}\text { ALPHA BRANCHING RATIO } & 1.00 \\ \text { EQUIVĀLENT PU EACTOR } & 0.00 \\ \text { FISSIIE_GRAM EQUIVALENT } & 1.00 \\ \text { SPECIFIC ACTIVITY } & 2.19 \mathrm{E}-6 \\ \text { THERMAL_FOWER } & 6.04 \mathrm{E}-8 \\ \text { X_SECTION } & 501.0 \\ \text { NU_BAR } & 2.42\end{array}$

END

\section{B17. OPTIONS.DAC}

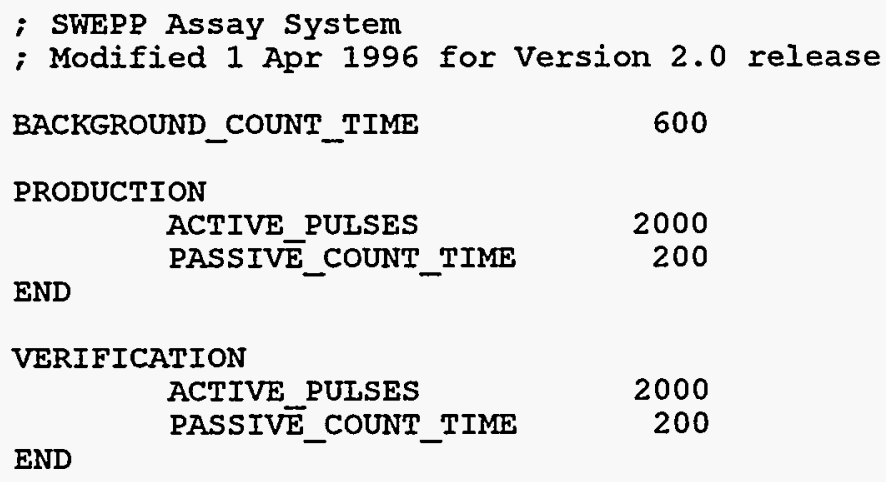




\section{B18. OPTIONS.CAC}

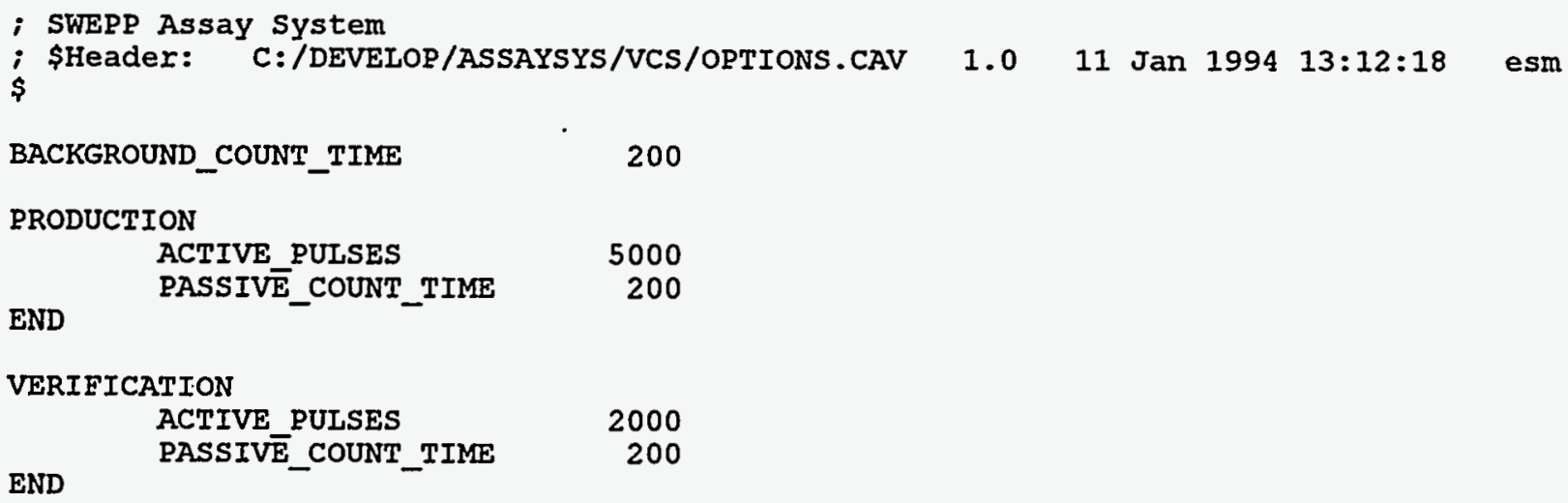

\section{B19. PASCRATE.DAC}

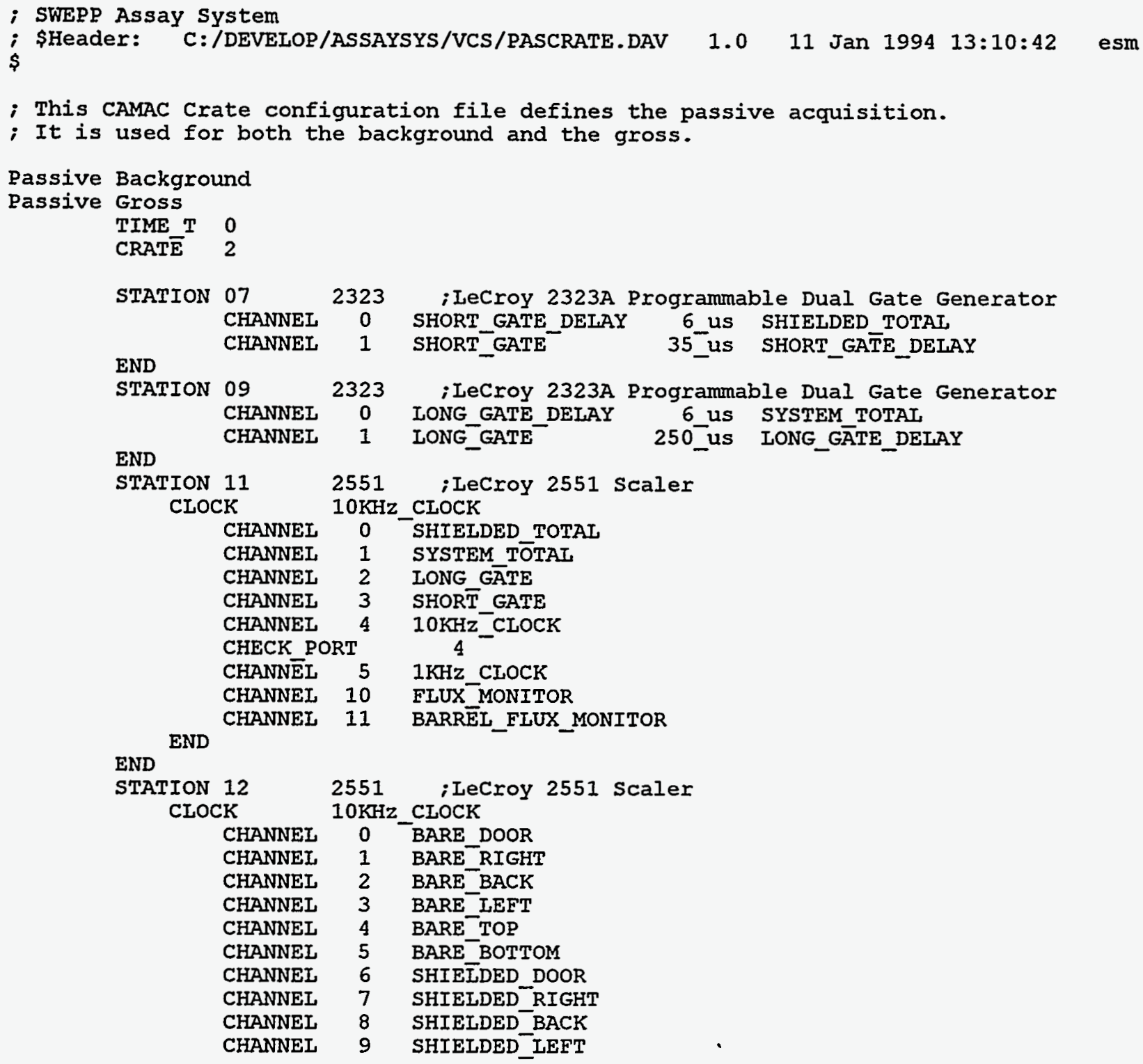




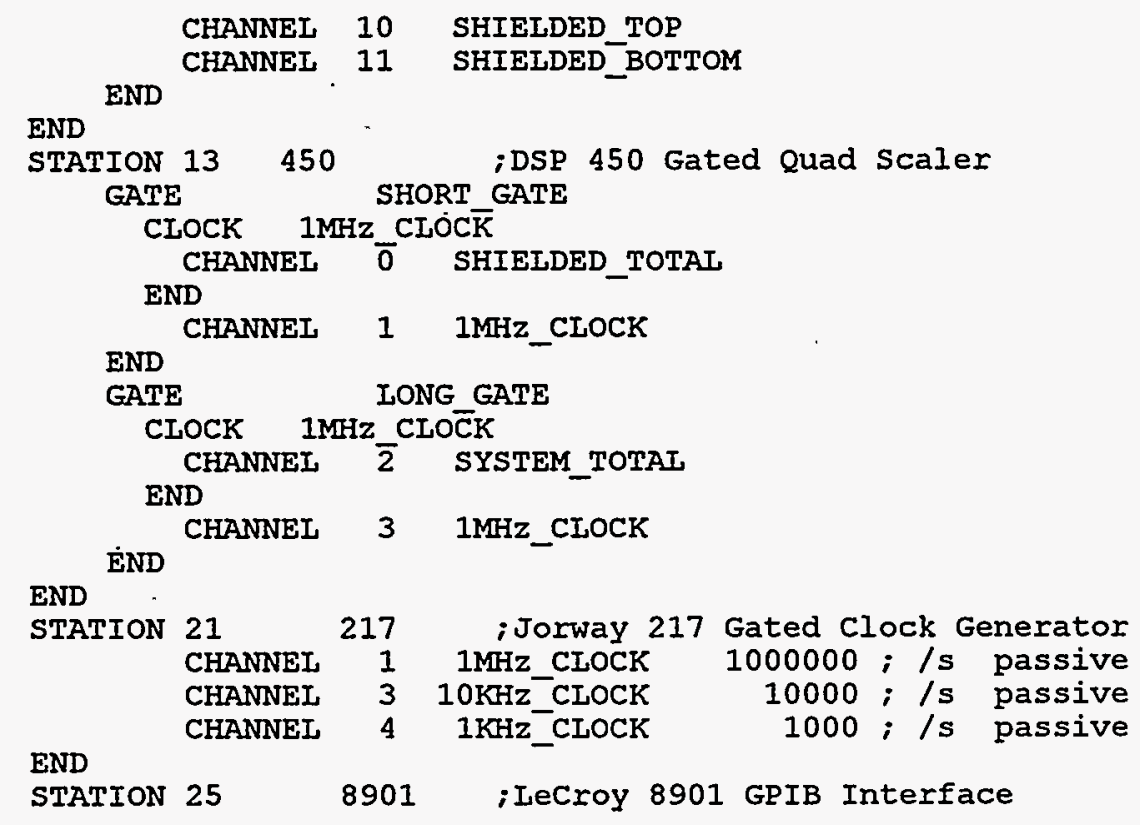

\section{B20. PASCRATE.CAC}

; SWEPP Assay System

; \$Header: C:/DEVELOP/ASSAYSYS/VCS/PASCRATE.CAV $\quad 1.0 \quad 11$ Jan 1994 13:12:18 esm $\$$

; This CAMAC Crate configuration file defines the passive acquisition.

; It is used for both the background and the gross.

Passive Background
Passive Gross
TIMET
CRATE

STATION 14 CHANNEL CHANNEI

END

STATION 16 CHANNEL CHANNEI

END

STATION 18 CLOCK

CHANNEL CHANNEL CHANNEL CHANNEL CHANNEL CHECK PORT CHANNEI 5 CHANNEL 10 END CHANNEL 11

END

STATION 19

CLOCK

CHANNEI CHANNEL

2323 iLecroy 2323A Programmable Dual Gate Generator SHORT_GATE_DEIAY 6_us SHIELDED_TOTAL SHORT_GATE

2323 iLecroy 2323A Programmable Dual Gate Generator IONG_GATE_DEIAY 6 US SYSTEM_TOTAI LONG_GATE ${ }^{250^{-} \text {Us IONG_GATE_DEIAY }}$

2551 iLecroy 2551 Scaler

$10 \mathrm{KHz}$ CLOCK

SHIELDED TOTAL

SYSTEM TOTAL

LONG GÄTE

SHORT GATE

10KHz_CLOCK

4

$1 \mathrm{KHz}$ CLOCK

FLUX MONITOR

BARREL_FLUX_MONITOR

$$
\begin{aligned}
& 2551 \text {; Lecroy } 2551 \text { scaler } \\
& 10 \mathrm{KHz} \text { CLOCK } \\
& 0 \text { BARE_DOOR } \\
& 1 \text { BARE_RIGHT }
\end{aligned}
$$




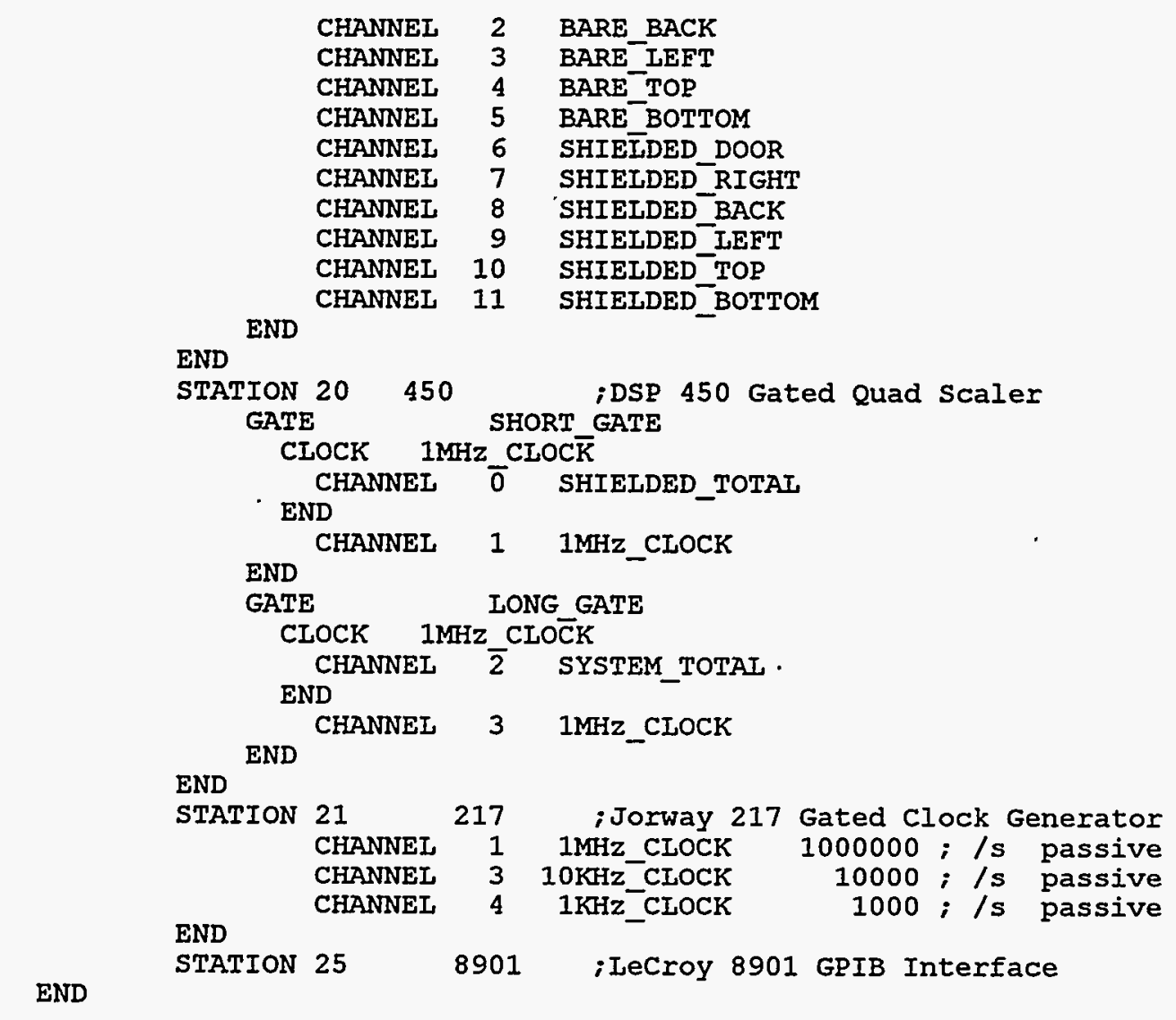

\section{B21. REGRESS.DAC}

; SWEPP Assay system

; \$Header: C:/DEVELOP/ASSAYSYS/VCS/REGRESS.DAV $\quad 1.0 \quad 11$ Jan $1994 \quad 13: 10: 44$ esm $\$$

; There are currently no regression test cases for the Drum Assay Chamber

;BACKGROUND "\develop\regress\background_file_name" ;TESTCASE

"\develop\regress\data_file_ñame"

\section{B22. REGRESS.CAC}

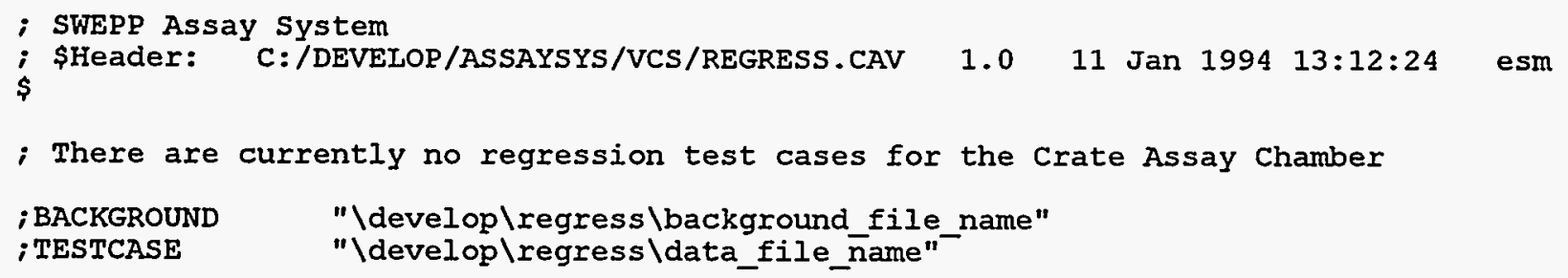




\section{B23. SAMPLE.001}

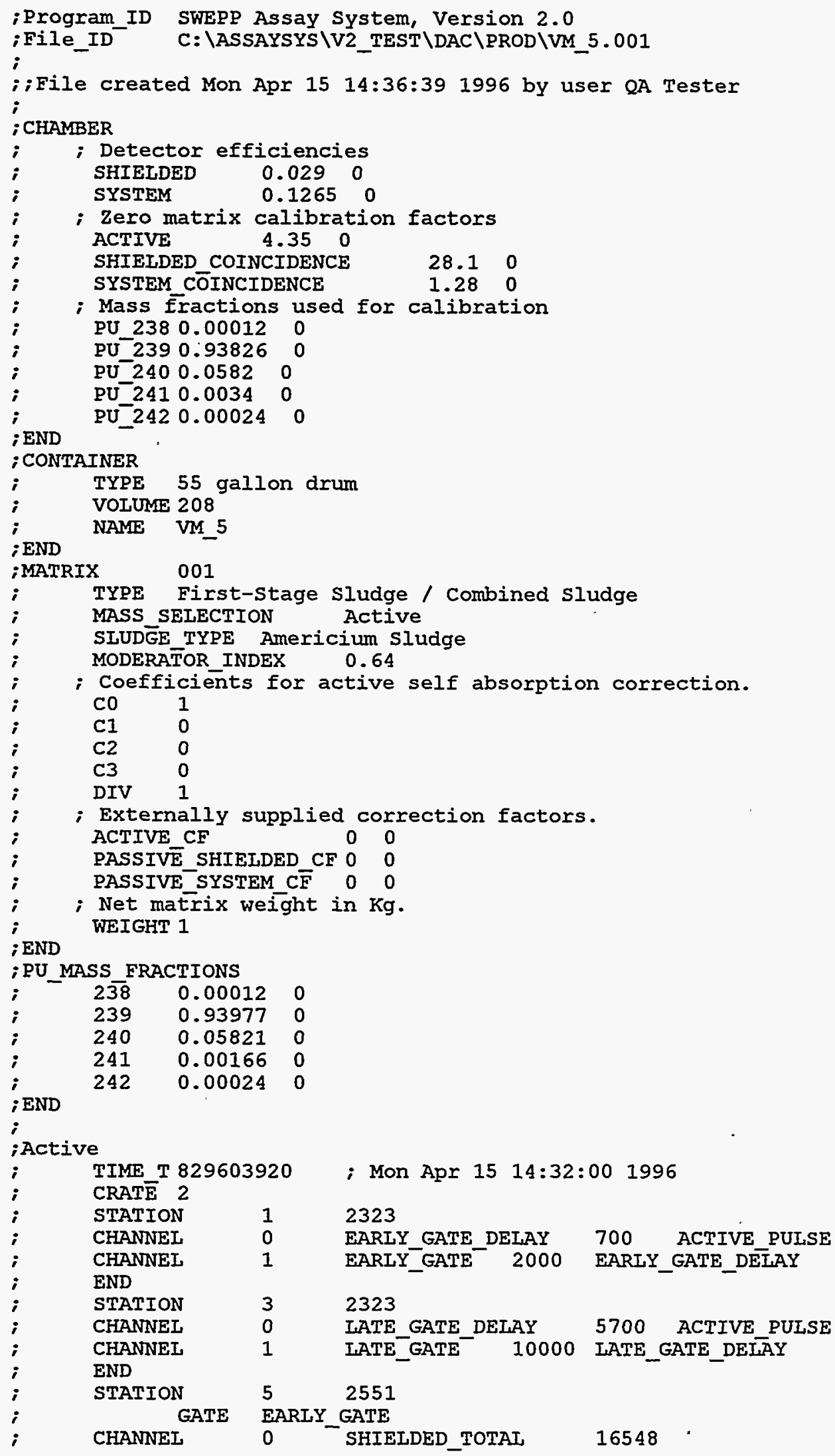




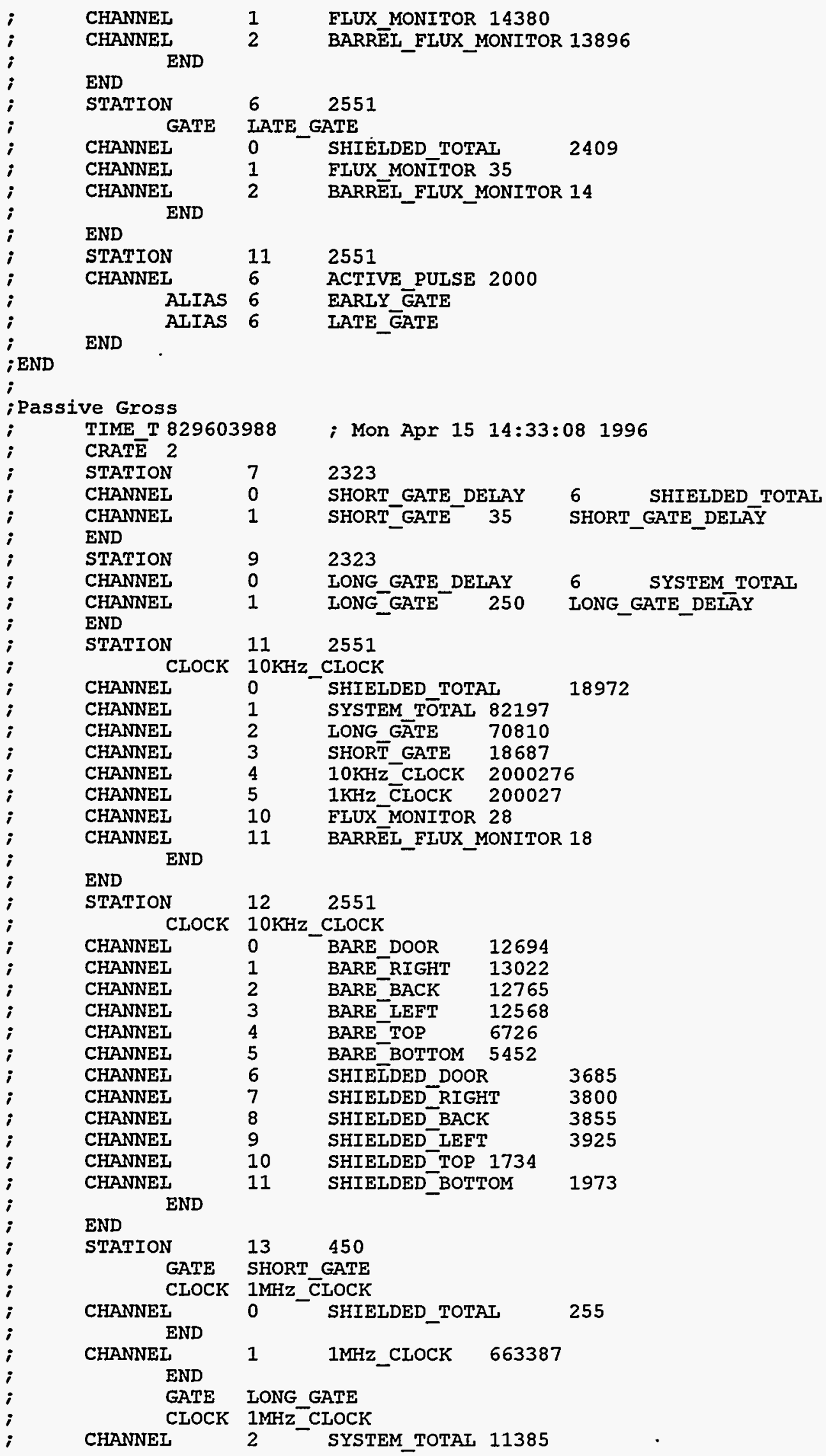




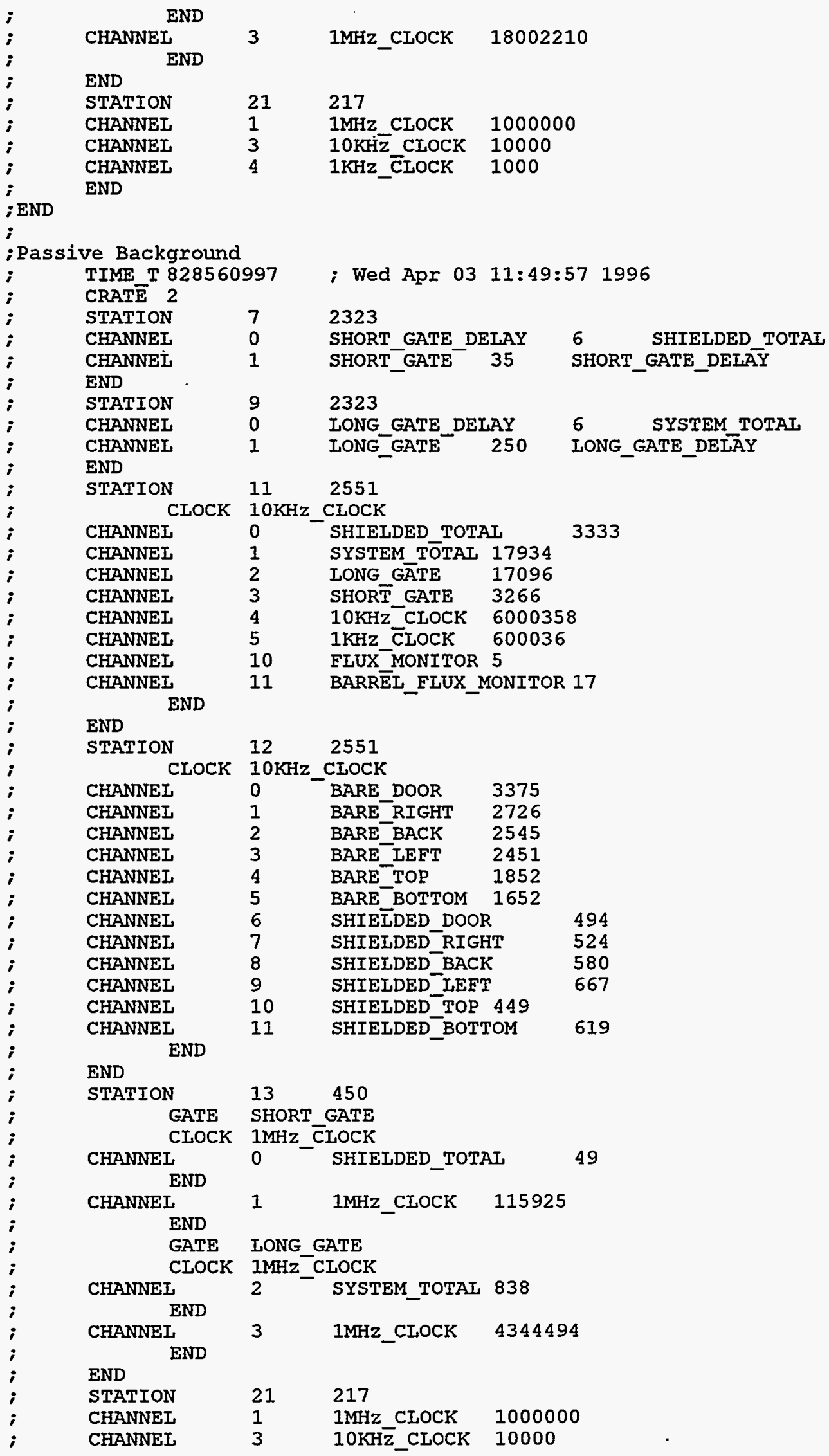




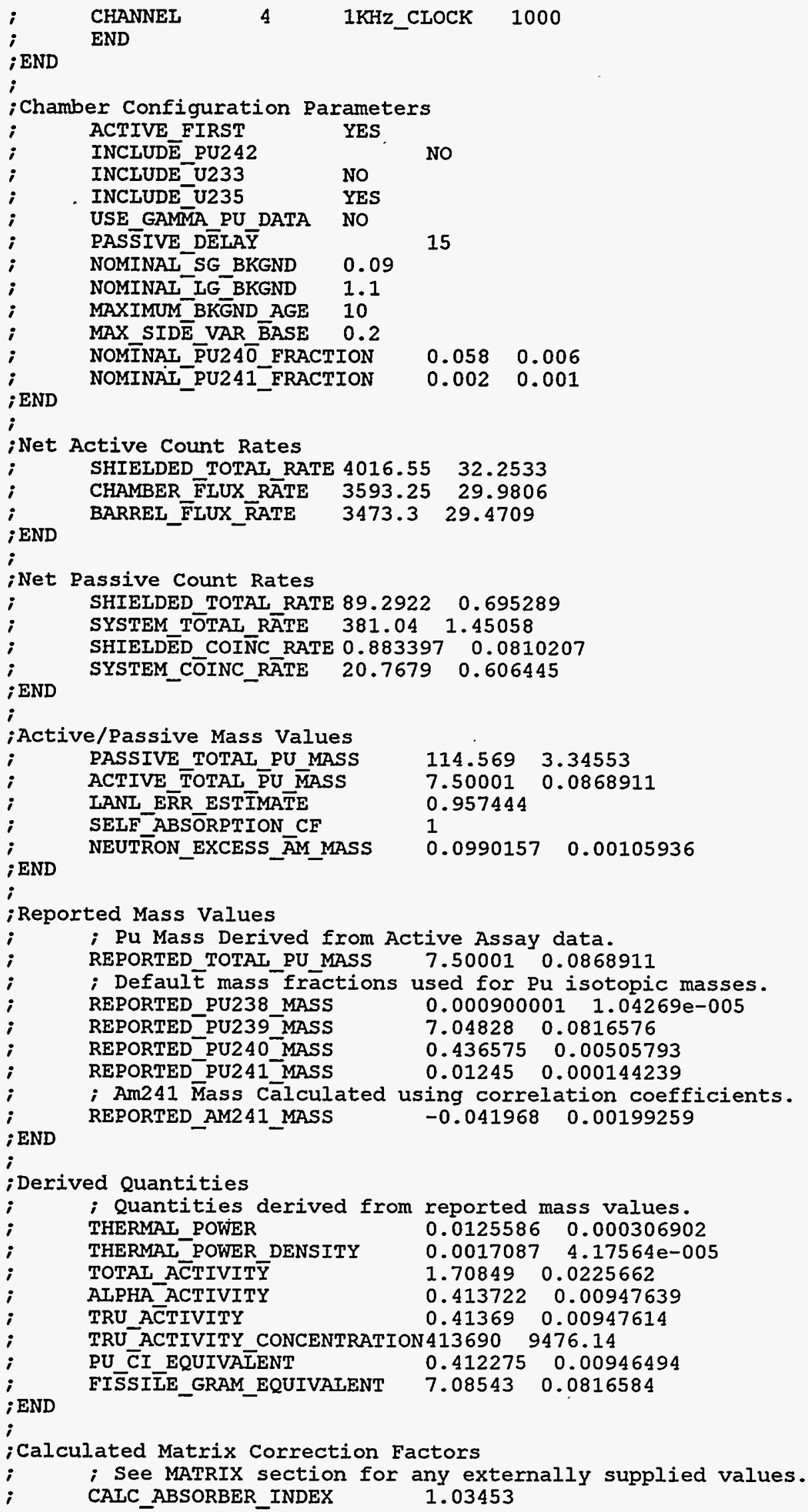




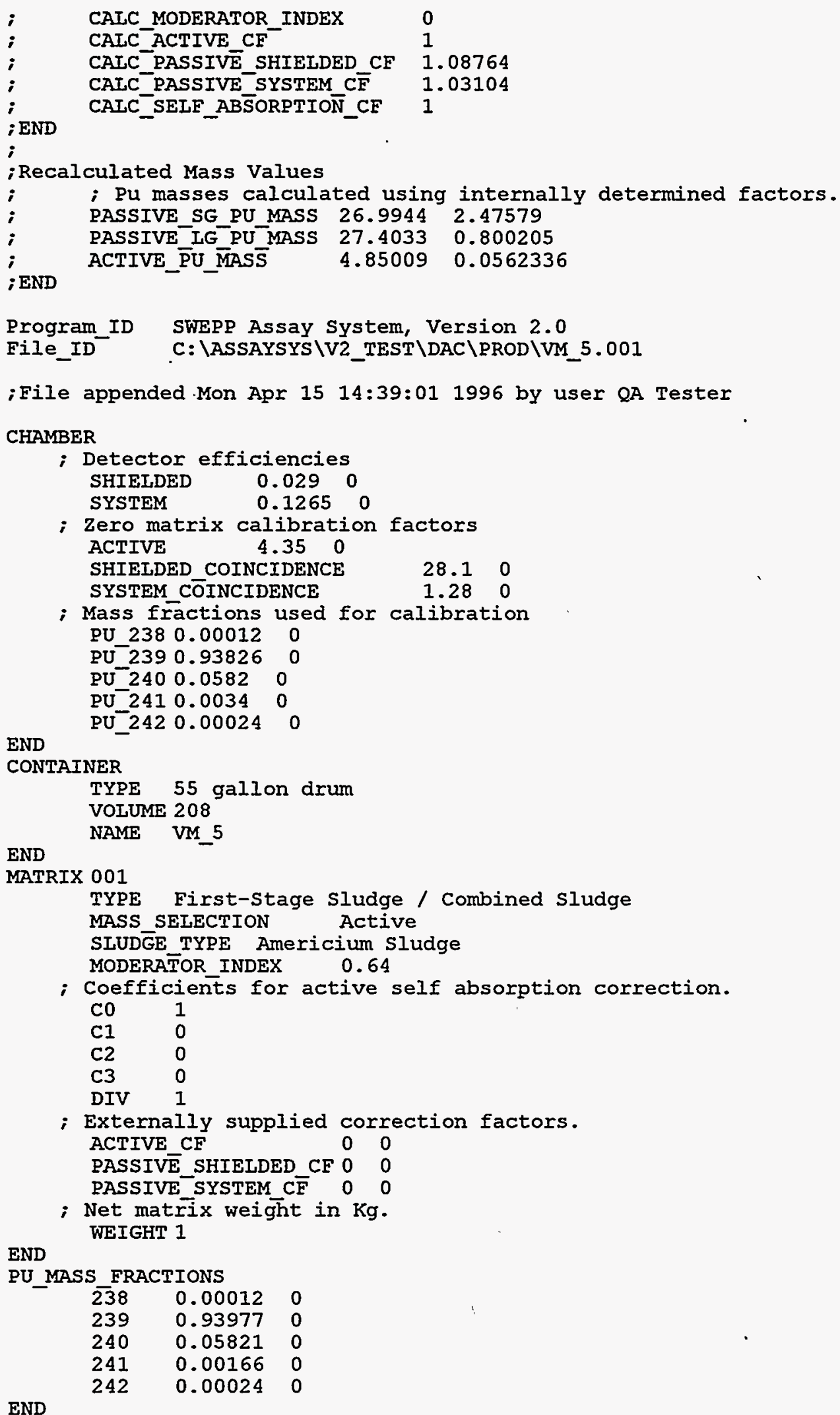

Active 


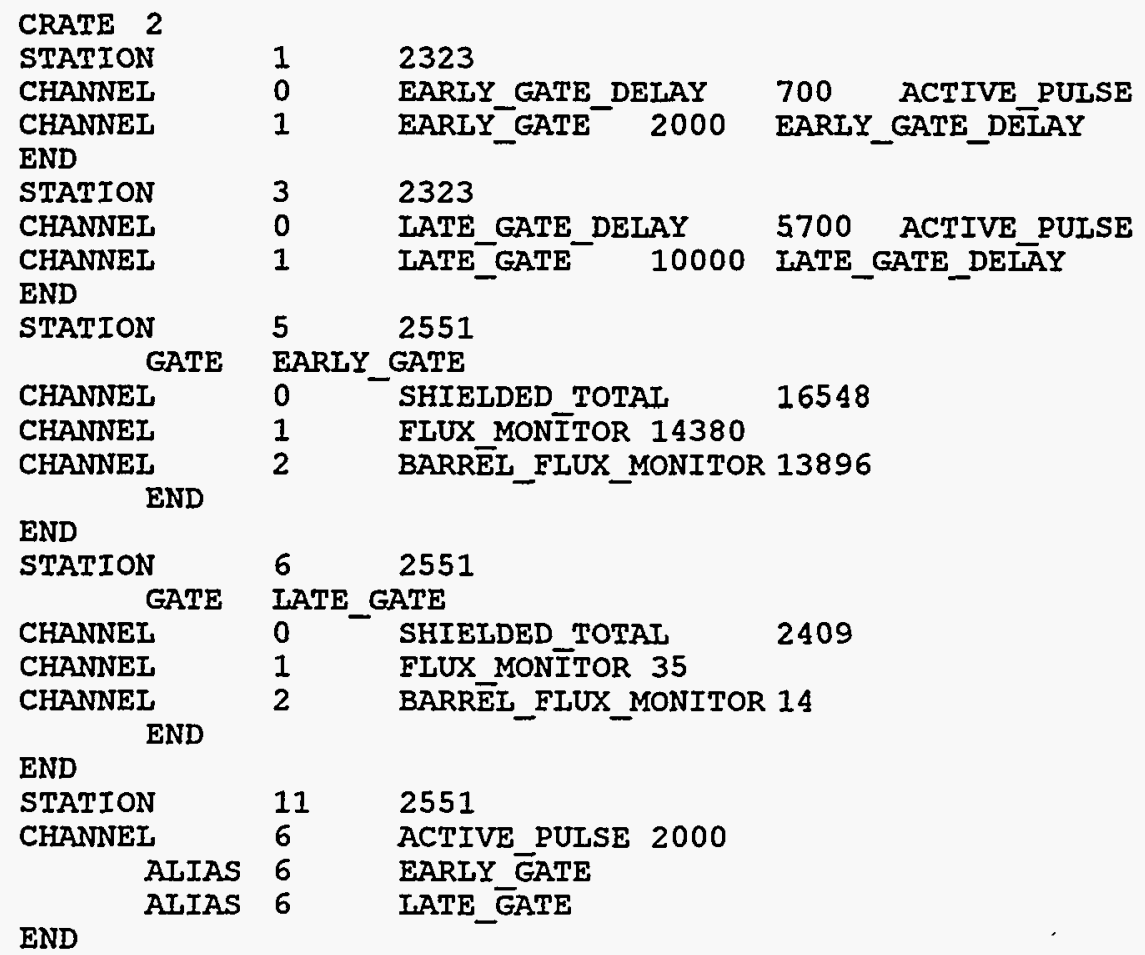

END

Passive Gross TIME_T 829603988 - ; Mon Apr 15 14:33:08 1996

CRATE 2 STATION CHANNEI CHANNEL END STATION CHANNEL CHANNEI, END 2323 SHORT GATE DELAY 6 SHIELDED TOTAI SHORT_GATE 35 SHORT_GATE_DELA $\overline{A Y}$

STATION CLOCK 10KHz_CLOCK

CHANNEI

CHANNEI

CHANNEI

CHANNEI

CHANNEI

CHANNEI

CHANNET

CHANNEI

END

92323

0 LONG GATE DELAY 6 SYSTEM TOTAL

1 LONG_GATE 250 LONG_GATE_DEIATY

END

STATION

CHANNEI

2551

CHANNEI

CHANNEI

CHANNEL

CHANNEI

CHANNEL

CHANNEI

CHANNEI

CHANNEI

CHANNEI

CHANNEL

CHANNEL

$$
0
$$

SHIELDED TOTAL SYSTEM TOTAL 82197 LONG GĀTE $\quad 70810$ SHORT GATE 18687 $10 \mathrm{KHz}$ CLOCK 2000276 $1 \mathrm{KHz}$ CLOCK 200027

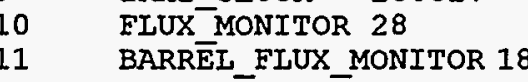

11 BARREI_FLUX_MONITOR 18

18972

IOKHz_CLOCK

0 BARE DOOR 12694

1 BARE_RIGHT 13022

2 BARE BACK 12765

3 BARE_LEFT 12568

4 BARE TOP 6726

5 BARE BOTTOM 5452

6 SHIEIDED DOOR 3685

7 SHIELDED_RIGHT 3800

8 SHIELDED BACK 3855

9 SHIELDED_IEFT 3925

10 SHIELDED TOP 1734

11 SHIELDED_BOTTOM 1973 . 


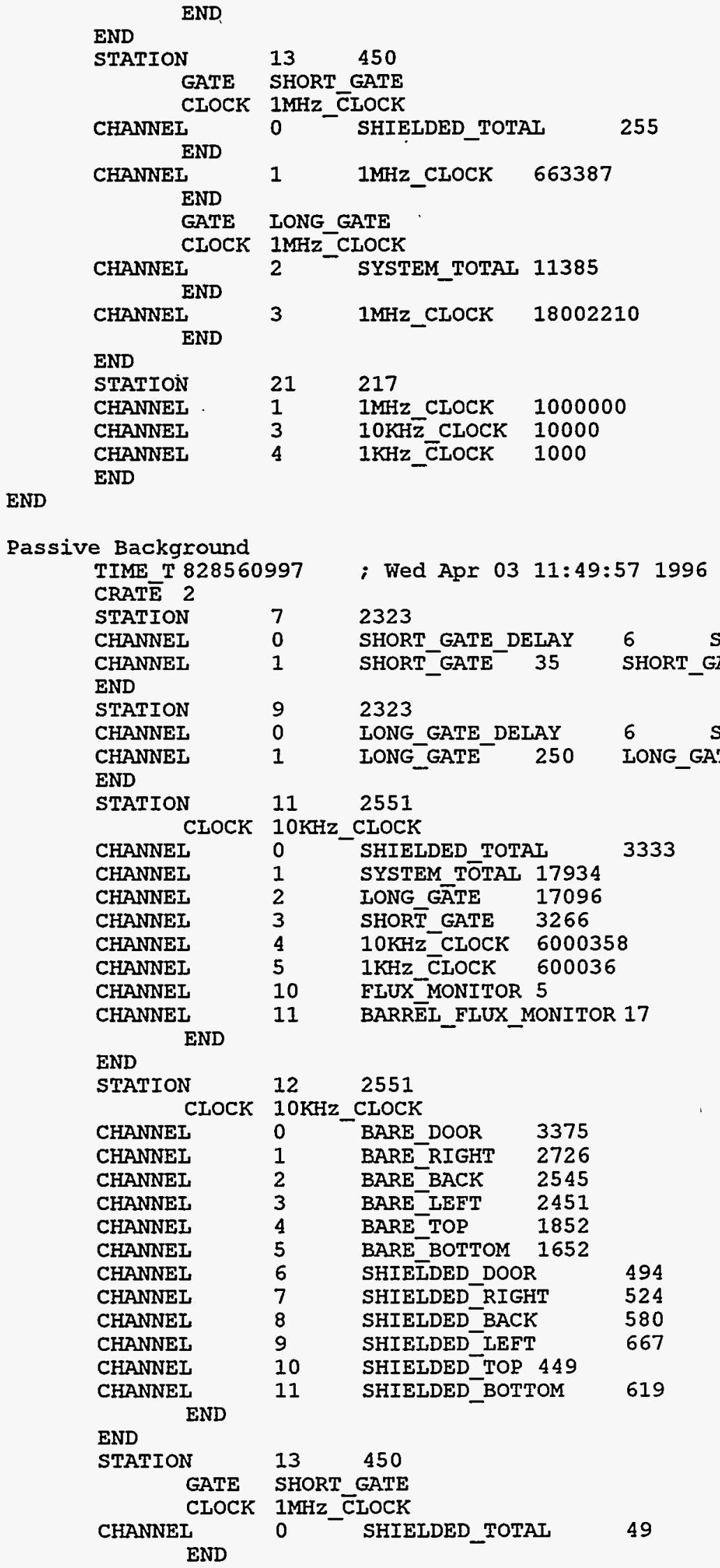




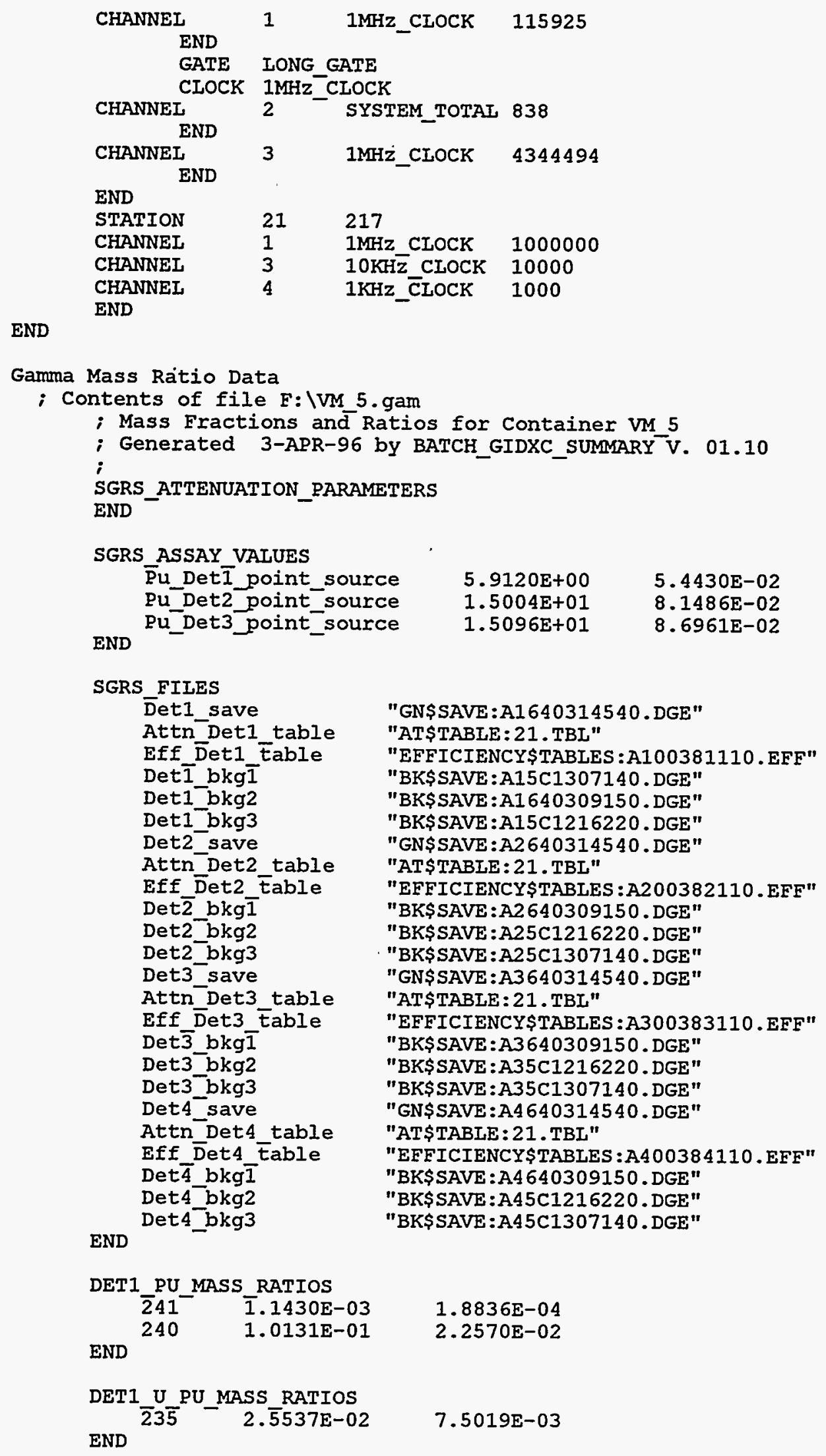




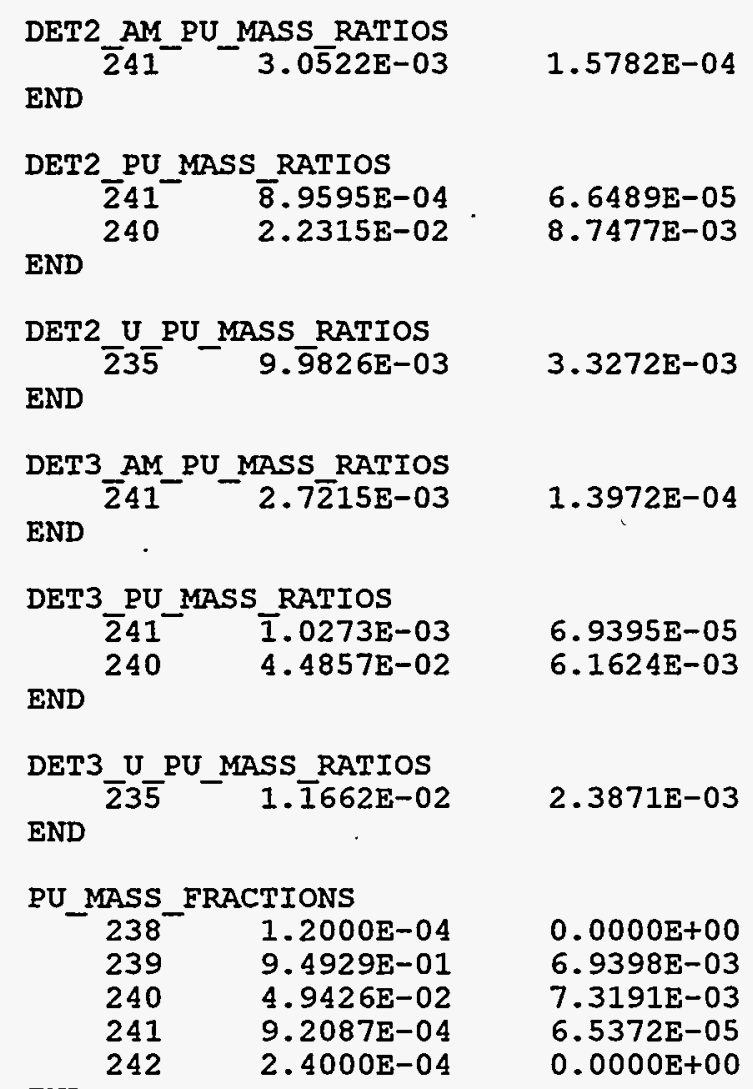

END

$\begin{array}{cll}\text { AVG_PU MASS RATIOS } & \\ 23 \overline{8} & 0.0000 \mathrm{E}+00 & 0.0000 \mathrm{E}+00 \\ 240 & 5.2066 \mathrm{E}-02 & 7.7007 \mathrm{E}-03 \\ 241 & 9.7006 \mathrm{E}-04 & 6.8497 \mathrm{E}-05 \\ 242 & 0.0000 \mathrm{E}+00 & 0.0000 \mathrm{E}+00\end{array}$

END

AVG AM PU MASS RATIOS

END

$$
24 \bar{I}-2 . \overline{8} 668 \mathrm{E}-03 \quad 1.6176 \mathrm{E}-04
$$

AVG U PU MASS RATIOS

$$
2 \overline{3} 51.1997 \mathrm{E}-02 \quad 1.8778 \mathrm{E}-03
$$

END

AVG AM U MASS RATIOS

$$
24 \overline{1} 0.0000 \mathrm{E}+00 \quad 0.0000 \mathrm{E}+00
$$

END

END

Chamber Configuration Parameters

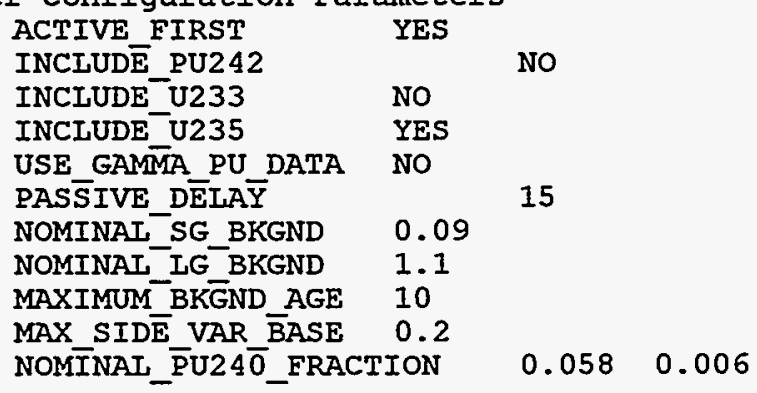


END

NOMINAL_PU241_ERACTION $0.002 \quad 0.001$

Net Active Count Rates

SHIEIDED TOTAL RATE 4016.55 32.2533

CHAMBER F̄LUX RĀTE $3593.25 \quad 29.9806$

END

BARREL_ELUX_FATE $\quad 3473.3 \quad 29.4709$

Net Passive Count Rates

SHIELDED TOTAL RATE 89.2922 0.695289

SYSTEM TOTTAL RÄTE $381.04 \quad 1.45058$

SHIELDED COI $\bar{N} C$ RATE $0.883397 \quad 0.0810207$

END

$\begin{array}{lll}\text { SYSTEM CŌINC RĀTE } 20.7679 & 0.606445\end{array}$

Active/Passive Mass Values

PASSIVE TOTAL PU MASS

$114.569 \quad 3.34553$

ACTIVE TOTAI_ $\overline{\mathrm{P} U}$ MASS

$\begin{array}{lll}7.4457 & 0.0866737\end{array}$

IANL ERR ESTTMATE

SELF_ABSORPTION_CF

URANIUM CF

0.950532

NEUTRON_EXCESS_AM_MASS

1

$1.00729 \quad 0.00114171$

END

0.09919120 .00105888

Reported Mass Values

; Pu Mass Derived from Active Assay data.

REPORTED_TOTAL_PU_MASS $7.4457 \quad 0.0866737$

; DefaulE mass Iräctions used for Pu isotopic masses.

REPORTED PU238 MASS $0.0008934831 .04008 \mathrm{e}-005$

REPORTED PU239 MASS $6.99724 \quad 0.0814533$

REPORTED_PU240_MASS $0.433414 \quad 0.00504528$

REPORTED PU241-MASS $\quad 0.0123599 \quad 0.000143878$

; U235 Máss Derived from Isotopic Ratio Data.

REPORTED U235 MASS 0.08394590 .0130805

; Am241 Mass Determined from Pu-239 Mass Ratio.

END

REPORTED AM2 4I MASS $0.0200597 \quad 0.00115571$

Derived Quantities

; Quantities derived from reported mass values.

$\begin{array}{llll}\text { THERMAL_POWER } & 0.0196277 & 0.000241934\end{array}$

THERMAI POWER DENSITY $\quad 0.00267049 \quad 3.2917 \mathrm{e}-005$

TOTAL_ACTIVITY $\bar{Y} \quad 1.91030 .0217992$

ALPHA ACTIVITY $\quad 0.624908 \quad 0.00760707$

$\begin{array}{lll}\text { TRU_ACTIVITY } & 0.624876 & 0.00760676\end{array}$

TRU ACTIVITY CONCENTRATION624876 7606.76

$\begin{array}{lll}\text { PU_CI_EQUIVA]ENT } & 0.623471 & 0.00759287\end{array}$

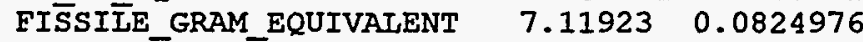

END

Calculated Matrix Correction Factors

; See MATRIX section for any externally supplied values.

CALC ABSORBER INDEX

CALC MODERATO $\bar{R}$ INDEX

CALC ACTIVE CF

1.03453

CATC ACTIVE CF

CALC_SELF_ABS̄ORPTION̄CF 1

END

Recalculated Mass Values

; Pu masses calculated using internally determined factors.

PASSIVE SG PU MASS 26.99442 .47579

PASSIVE IG PU MASS $27.4033 \quad 0.800205$

END

ACTIVE_DU_MASS

$4.81496 \quad 0.0560925$ 